\title{
Métodos de estimação baseados na função de verossimilhança para modelos lineares elípticos
}

\author{
Natalia Andrea Milla Pérez
}

TESE APRESENTADA

$\mathrm{AO}$

Instituto De MatemáticA E EstatísticA

DA

UNIVERSIDADE DE SÃo PAUlO

PARA

OBTENÇÃO DO TÍTULO

$\mathrm{DE}$

Doutor EM Cî̂NCIAS

Programa: Doutorado em Estatística

Orientador: Prof. Dr. Gilberto Alvarenga Paula

Durante o desenvolvimento deste trabalho o autor recebeu auxílio financeiro da CAPES(Brasil) e CONICYT(Chile)

São Paulo, novembro de 2018 


\section{Resumo}

PEREZ, N. A. M. Métodos de estimação baseados na função de verossimilhança para modelos lineares elípticos. 2018. 154 f. Tese (Doutorado) - Instituto de Matemática e Estatística, Universidade de São Paulo, São Paulo, 2018.

O objetivo desta tese é estudar métodos de estimação baseados na função de verossimilhança em modelos mistos lineares elípticos. Derivamos inicialmente os métodos de máxima verossimilhança, máxima verossimilhança restrita e de máxima verossimilhança perfilada modificada para o modelo linear normal. Estendemos os métodos para os modelos lineares elípticos e encontramos diferenças entre as equações resultantes de cada método. A principal motivação deste trabalho é que o método de máxima verossimilhança restrita tem sido aplicado para obter estimadores menos viesados para os componentes de variância-covariância, em contraste com os estimadores de máxima verossimilhança. O método tem sido muito utilizado em modelos com estruturas de variância-covariância, como é o caso dos modelos mistos lineares. Assim, procuramos estender o método para os modelos mistos lineares elípticos bem como comparar com outros procedimentos de estimação, máxima verossimilhança e máxima verossimilhança perfilada modificada. Estudamos em particular os modelos mistos lineares com erros t-Student e exponencial potência.

Palavras-chave: máxima verossimilhança restrita, máxima verossimilhança perfilada modificada, métodos robustos, modelos linerares elípticos, modelos mistos, modelos exponencial potência, modelos t-Student. 


\section{Abstract}

PEREZ, N. A. M Estimation methods based on the likelihood function in Elliptical Linear Models. 2018. 154 f. Tese (Doutorado) - Instituto de Matemática e Estatística, Universidade de São Paulo, São Paulo, 2018.

The aim of this thesis is to study estimation methods based on the likelihood functions in elliptical linear mixed models. First, we review the modified profile maximum likelihood and the restricted maximum likelihood methods as well as the traditional maximum likelihood method in normal linear models. Then, we extend the methodologies for elliptical linear models and we compare the estimating equations derived for each method. The main motivation of the work is that the restricted maximum likelihood method has been largely applied in normal linear mixed models in order to reduce the bias of the maximum likelihood variance-component estimators. So, we intend to investigate the possible extension for elliptical linear mixed models as well as to compare with the modified profile maximum likelihood and the maximum likelihood methods. Particular studies for Student-t and power exponential linear mixed models are presented.

Keywords: linear elliptical models, mixed models, modified profile maximum likelihood, power exponencial models, restricted maximum likelihood, robust methods, Student-t models. 


\section{Sumário}

Lista de Figuras $\quad$ xi

Lista de Tabelas $\quad$ xiii

1 Introdução $\quad 1$

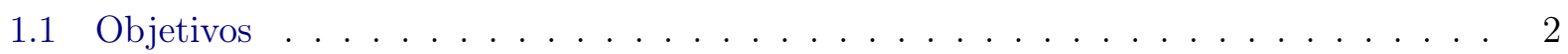

1.2 Organização do Trabalho . . . . . . . . . . . . . . . . . . . . 2

2 Estimação baseada na função de verossimilhança para o modelo linear normal 5

2.1 Modelo linear normal . . . . . . . . . . . . . . . . . . . . . . . . . 5

2.2 Estimação de máxima verossimilhança . . . . . . . . . . . . . . . . . 6

2.3 Estimação de máxima verossimilhança restrita . . . . . . . . . . . . . . . . . . 6

2.3.1 Método proposto por Verbyla . . . . . . . . . . . . . . . . . 7

2.3.2 Transformação do modelo proposto . . . . . . . . . . . . . . . . . . . 7

2.3.3 Derivação dos estimadores de máxima verossimilhança restrita $\quad \ldots . \ldots .9$

2.4 Estimação usando a função de verossimilhança perfilada modificada . . . . . . . . . . . 10

2.4.1 Derivação do estimador de máxima verossimilhança perfilada modificada . . . 10

2.5 Caso particular da matriz de variância-covariância . . . . . . . . . . . . . . . . 11

2.6 Comentários . . . . . . . . . . . . . . . . . . . . . . . . . . 13

3 Métodos de estimação no modelo linear elíptico $\quad 15$

3.1 Modelo linear elíptico f . . . . . . . . . . . . . . . . . . . . . . . . . 15

3.2 Estimação de máxima verossimilhança . . . . . . . . . . . . . . . . . . . . 16

3.3 Estimação de máxima verossimilhança restrita . . . . . . . . . . . . . . . . . . . . 17

3.3 .1 Método proposto por Verbyla . . . . . . . . . . . . . . . . . . . 18

3.4 Estimação baseada na verossimilhança perfilada modificada . . . . . . . . . . . . . . 21

3.4.1 Ajuste da função de verossimilhança perfilada modificada . . . . . . . . . . . 21

3.4.2 Ajuste da função verossimilhança perfilada modificada aproximada . . . . . . 22

3.5 Casos particulares para a matriz de escala . . . . . . . . . . . . . . . . . 24

3.5.1 Estrutura da matriz escala simples . . . . . . . . . . . . . . . . . . 24

3.5.2 Estrutura linear para a matriz escala . . . . . . . . . . . . . . 25

3.5.3 Outro tipo de estrutura linear com dois parâmetros . . . . . . . . . . . . . . 27

3.5.4 Estrutura para a matriz escala num modelo autoregressivo . . . . . . . . . . 30

3.6 Casos particulares de distribuições elípticas . . . . . . . . . . . . . . . . . . . . . 31

3.6.1 Modelo linear t-Student . . . . . . . . . . . . . . . . . . . . . 32 
3.6.2 Modelo linear exponencial potência . . . . . . . . . . . . . . . . . . . 36

3.7 Comentários . . . . . . . . . . . . . . . . . . . . . . . . 39

4 Métodos de estimação no modelo misto linear elíptico 41

4.1 Modelo misto linear elíptico . . . . . . . . . . . . . . . . . . . . . . . . . . 41

4.2 Métodos de estimação . . . . . . . . . . . . . . . . . . . . . . . . 42

4.2 .1 Estimação de máxima verossimilhança . . . . . . . . . . . . . . . . . . . 43

4.2 .2 Estimação de máxima verossimilhança restrita . . . . . . . . . . . . . . . . . 44

4.2.3 Estimação baseada na verossimilhança perfilada modificada . . . . . . . . . . 46

4.3 Casos particulares de distribuições elípticas . . . . . . . . . . . . . . . . . . . . 49

4.3.1 Modelo misto linear t-Student . . . . . . . . . . . . . . . . . . . . . . . 49

4.3.2 Modelo misto linear exponencial potência . . . . . . . . . . . . . . . . . 52

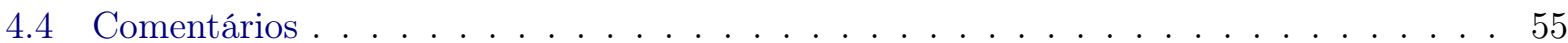

5 Procedimento alternativo de estimação restrita $\quad 57$

5.1 Formulação da função de verossimilhança restrita . . . . . . . . . . . . . . . . . . . 57

5.2 Aproximação da verossimilhança marginal . . . . . . . . . . . . . . . . . 58

5.2 .1 Estimação dos efeitos fixos . . . . . . . . . . . . . . . . . . . . . . 59

5.2 .2 Estimação dos parâmetros de variância e covariância . . . . . . . . . . . . . 60

5.3 Comentários . . . . . . . . . . . . . . . . . . . . . 60 60

6 Simulações e Aplicações $\quad 63$

6.1 Simulações do modelo linear t-Student . . . . . . . . . . . . . . . . . . 63

6.1 .1 Estrutura simples para a matriz escala . . . . . . . . . . . . . . . 63

6.1.2 Estrutura autoregressiva para a matriz escala . . . . . . . . . . . . . . 64

6.2 Simulações para o modelo misto linear t-Student . . . . . . . . . . . . . . . . 67

6.2 .1 Modelo misto linear t-Student balanceado . . . . . . . . . . . . . . . . . . 67

6.3 Aplicações . . . . . . . . . . . . . . . . . . . . . . . . . . 74

6.3.1 Volume do Plasma . . . . . . . . . . . . . . . . . . . . . . . . . 74

6.3.2 Dados censitários de Boston . . . . . . . . . . . . . . . . . . . . . 77

6.4 Comentários . . . . . . . . . . . . . . . . . . . . . . 83

7 Discussões $\quad 85$

7.1 Considerações Finais . . . . . . . . . . . . . . . . . . . . . . 85

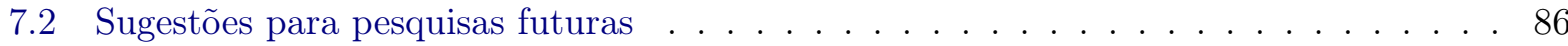

$\begin{array}{ll}\text { A Conceitos preliminares } & \mathbf{8 7}\end{array}$

A.1 Método de estimação restrita . . . . . . . . . . . . . . . . . . . . 87

A.2 Função de verossimilhança perfilada modificada . . . . . . . . . . . . . . . . . 88

A.2.1 Método de estimação baseado na função de verossimilhança perfilada . . . . . 89

B Cálculos de Matrizes $\quad 93$

B.1 Fatorizações de Matrizes . . . . . . . . . . . . . . . . . . . . 93

B.1.1 Termos para a distribuição condicional $\mathbf{V}_{1} \mid \mathbf{V}_{2} \ldots \ldots \ldots$. . . . . . . . . 93

B.1.2 Outras fatorizações . . . . . . . . . . . . . . . . . . . . . . 94 
B.2 Cálculos das derivadas de um elemento do vetor $\boldsymbol{\alpha} \ldots \ldots \ldots$. . . . . . . . 94

B.3 Cálculos das derivadas da matriz escala . . . . . . . . . . . . . . . . . . 95

$\begin{array}{ll}\text { C Distribuição elíptica } & 105\end{array}$

C.1 Definição da família elíptica . . . . . . . . . . . . . . . . . . . . . . 105

C.2 Propriedades da família elíptica . . . . . . . . . . . . . . . . . . 105

$\begin{array}{ll}\text { D Modelo linear misto t-Student } & 109\end{array}$

D.1 Equações de estimação . . . . . . . . . . . . . . . . . . . . . . . . . 109

D.2 Cálculos e derivadas . . . . . . . . . . . . . . . . . . . . . . 110

D.2.1 Matriz de informação . . . . . . . . . . . . . . . . . . . . . . . 110

D.2.2 Matriz de informação esperada para $\boldsymbol{\beta}$. . . . . . . . . . . . . . . . 110

D.2.3 Derivadas parciais de $u_{i 2}$ e $\mathbf{b}_{i}$ com respeito a $\theta_{j} \ldots \ldots \ldots \ldots 11$

D.2.4 Derivadas parciais de $\hat{u}_{i}$ e $\hat{\boldsymbol{\beta}}_{\theta}$ com respeito a $\theta_{j} \ldots \ldots \ldots \ldots 11$

D.3 Modelos simulados . . . . . . . . . . . . . . . . . . . . . . . 111

D.3.1 Estrutura autoregressiva . . . . . . . . . . . . . . . . . 111

D.3.2 Modelo misto linear t-Student . . . . . . . . . . . . . . . . . . . 113

$\begin{array}{ll}\text { E Aproximação parcial de Laplace } & 117\end{array}$

$\begin{array}{llr}\text { F Tabelas } & 121\end{array}$

F.1 Tabelas de resultados das aplicações . . . . . . . . . . . . . . . . . . 121

$\begin{array}{lr}\text { Referências Bibliográficas } & 123\end{array}$

Listas de figuras e tabelas criadas automaticamente 


\section{Lista de Figuras}

6.1 Gráficos boxplots para as estimativas de $\sigma^{2}$, considerando um modelo linear t-Student com estrutura autoregressiva para a matriz escala, os verdadeiros valores para $\sigma^{2}=1$ e $\rho=0.5$.

6.2 Gráficos boxplots para as estimativas de $\rho$, considerando um modelo linear t-Student com estrutura autoregressiva para a matriz escala, os verdadeiros valores para $\sigma^{2}=1$ e $\rho=0.5$. 69

6.3 Gráficos boxplots para as estimativas de $\alpha$, considerado um modelo misto linear t-Student balanceado com valores dos parâmetros $\alpha=5$ e $\gamma=5$. . . . . . . . . . . . . . . . . . . . 70

6.4 Gráficos boxplots para as estimativas de $\gamma$, considerado um modelo misto linear t-Student balanceado com valores dos parâmetros $\alpha=5$ e $\gamma=5$. . . . . . . . . . . . . . . . . . . . 71

6.5 Gráficos de comparação do volume do plasma obtidos pelos métodos Hurley e Nalder. . . 75

6.6 qqplots das distâncias transformadas, a) modelo normal e b) modelo t-Student. . . . . . . . 77

6.7 Gráficos boxplots das variáveis LMV, CRIME, ROOM e AGE com respeito aos distritos. . . 79

6.8 Gráficos boxplots das variáveis DIST, BLACK, LSTAT e NOXSQ, com respeito aos distritos. 80

6.9 Gráficos de dispersão das variáveis CRIME, ROOM, AGE e DIST em relação com à variável LMV. . . . . . . . . . . . . . . . . . . . . . . . . . . . . . . . 81

6.10 Gráficos de dispersão das variáveis BLACK, LSTAT, CHAS e NOXSQ em relação com à variável LMV. . . . . . . . . . . . . . . . . . . . . . . . . . . . . . . . . . . . 82

6.11 qqplots das distâncias transformadas, a) modelo normal e b) modelo t-Student. . . . . . . . 83 


\section{Lista de Tabelas}

3.1 Resumo de algumas distribuições pertencentes à família elíptica. . . . . . . . . . . . 16

3.2 Expressões de $w_{g}(u)$ e $w_{g}^{\prime}(u)$ para algumas distribuições pertencentes à família elíptica. 32

6.1 Estimativas de $\sigma^{2}$ obtidas pelos três métodos de estimação, para $\sigma^{2}=1 \ldots$. . . . . . 64

6.2 Estimativas de $\sigma^{2}$ obtidas por diferentes métodos de estimação, considerando o valor

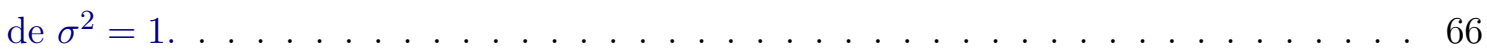

6.3 Estimativas de $\rho$ obtidas por diferentes métodos de estimação, considerando o valor

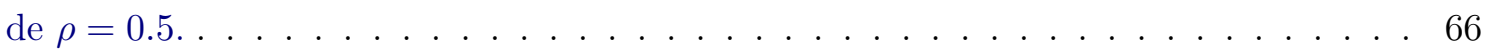

6.4 Estimativas de $\alpha$ e $\gamma$ obtidas pelos diferentes métodos de estimação, considerando o modelo misto linear t-Student balanceado $(m=5)$ com valores de $\alpha=5$ e $\gamma=5$. . $\quad 72$

6.5 Viés relativo das estimativas de $\alpha$ e $\gamma$ obtidas pelos diferentes métodos de estimação. Considerando amostras gerados de uma distribuição t-Student, com $m$ de tamanhos 5,15 e 45 e valores de $\alpha=5$ e $\gamma=5 \ldots \ldots \ldots \ldots \ldots \ldots$

6.6 Viés relativo das estimativas de $\alpha$ e $\gamma$ obtidas pelos diferentes métodos de estimação. Considerando amostras gerados uma distribuição normal, com $m$ (grupos) de tamanhos 5, 15 e 45, tamanhos de amostra 25, 100, 200 e 800, para valores de $\alpha=5$ e

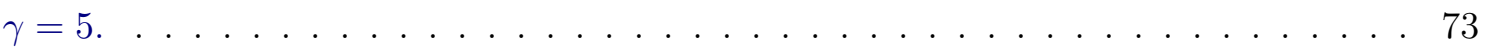

6.7 Escolha dos graus de liberdade. . . . . . . . . . . . . . . . . . 75

6.8 Resultados das estimativas considerando o modelo normal e o modelo t-Student. ${ }^{a}$. . 76

6.9 Estimativas bootstrap dos parâmetros para o modelo normal e modelo t-Student, obtidas pelos diferentes métodos de estimação. . . . . . . . . . . . . . . . . . . . . . . . . 77

6.10 Descrição das variáveis dados censitários de Boston. . . . . . . . . . . . . . . 78

6.11 Estatísticas descritivas da variável dependente LMV para os 15 distritos considerados. 79

6.12 Valores do critério de informação para a distribuição t-Student com diferentes graus

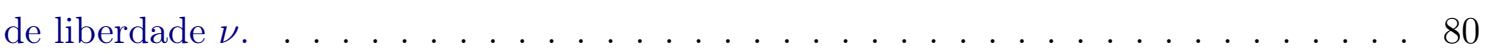

6.13 Estimativas dos parâmetros considerando o modelo normal e o modelo t-Student, obtidas pelos diferentes métodos de estimação. ${ }^{b} \ldots \ldots \ldots \ldots$. . . . . . . 82

F.1 Estimativas bootstrap dos parâmetros e seus desvio padrão para o modelo normal e modelo t-Student, obtidas pelos diferentes métodos de estimação, considerando o procedimento de reamostragem em que todos os distritos têm a mesma probabilidade de ser escolhido. . . . . . . . . . . . . . . . . . . . . . . 121 
F.2 Estimativas bootstrap dos parâmetros e seus desvio padrão para o modelo normal e modelo t-Student, obtidas pelos diferentes métodos de estimação, considerando o procedimento de reamostragem em que os distritos de maior tamanho têm uma maior probabilidade de ser escolhido. . . . . . . . . . . . . . . . . . . . . 122 


\section{Capítulo 1}

\section{Introdução}

Na literatura Estatística há diferentes métodos de estimação de parâmetros, sendo um dos mais utilizados o método de máxima verossimilhança, devido às propriedades de seus estimadores e a seu desenvolvimento em diferentes áreas de aplicação. Esses métodos, geralmente, surgem da complexidade da estimação dos parâmetros ou da motivação em encontrar um melhor estimador dos parâmetros de interesse num modelo determinado.

A estimação de máxima verossimilhança restrita é um método de estimação baseado numa transformação da função de verossimilhança dos dados originais. A primeira ideia deste tipo de estimação foi apresentada por Patterson e Thompson (1971) num modelo linear normal de blocos incompletos. Os autores propuseram uma transformação linear da variável resposta permitindo estimar de forma separada os parâmetros da média e os parâmetros da matriz de variância-covariância. Em outras palavras, a transformação permite escrever o logaritmo da função verossimilhança como uma soma de dois logaritmos de funções de verossimilhanças. Além de facilitar a estimação dos parâmetros, a transformação proporciona estimadores não viesados para os parâmetros da matriz de variância-covariância do modelo estudado.

Do trabalho de Patterson e Thompson (1971), porém sob um ponto de vista Bayesiano, Harville (1974) mostra que a mesma transformação permite escrever a função de verossimilhança como o produto de duas funções de verossimilhanças: uma marginal que depende apenas dos parâmetros da matriz de variância-covariância e outra condicional para estimar os parâmetros da média de uma distribuição normal. Este enfoque tem a mesma interpretação da construção de funções de pseudo-verossimilhança por marginalização ou condicionamento, metodologia que é utilizada para estimar os parâmetros de interesse eliminando os parâmetros de incômodo (uma discussão desta metodologia é apresentada, por exemplo, em Basu (1977)).

A partir das condições estabelecidas por Patterson e Thompson (1971) para a transformação, Harville (1977) prova que para qualquer matriz que satisfaz tais condições, a estrutura da transformação resultante é a mesma para o modelo linear normal. Outro trabalho que consideramos neste estudo foi apresentado por Verbyla (1990) para um modelo linear normal, com um enfoque similar ao de Harville (1977). Satisfazendo às propriedades da transformação de Patterson e Thompson (1971), mas sem especificar completamente a matriz da transformação, Verbyla (1990) consegue escrever o logaritmo da função de verossimilhança como a soma de dois logaritmos de funções de verossimilhanças. A partir desses artigos surgiram vários trabalhos relacionados com transformações da variável resposta, sendo que alguns autores denominam esta metodologia de estimação de máxima verossimilhança restrita (REML) ou estimação de máxima verossimilhança residual. A transformação também tem sido denominada como contraste dos erros. Dentre os autores que desenvolveram ou aplicaram esta metodologia em diversos modelos temos, por exemplo, Copper e Thompson (1977) em modelos de séries temporais, Corbeil e Searle (1976) e Demidenko (2013) em modelos mistos lineares normais, McGilchrist e Yau (1995) em modelos mistos lineares generalizados, Cressie (1993) em modelos espaciais e Verbeke e Molenberghs (2000) em modelos mistos lineares para dados longitudinais.

Por outra parte, temos as distribuições elípticas como uma família de distribuições multivaria- 
das simétricas que contém a distribuição normal multivariada e outras distribuições de caudas mais pesadas ou mais leves, tais como t-Student, exponencial potência, normal contaminada, logística I, logística II, slash, dentre outras (ver Fang et al. (1990)). Vários trabalhos foram publicados nas últimas décadas relacionados com modelos lineares e não lineares elípticos. Por exemplo, Lange et al. (1989) propuseram o modelo multivariado t-Student com graus de liberdade conhecidos ou desconhecidos, Welsh e Richardson (1997) derivaram o modelo multivariado t-Student marginal, enquanto que Lindsay (1999) discutiu o modelo multivariado exponencial potência para a análise de medidas repetidas e Pinheiro et al. (2001) expressaram o modelo linear misto t-Student como um modelo de estrutura de escala normal e propuseram um processo iterativo para a estimação conjunta dos parâmetros. Savalli et al. (2006) propuseram um modelo misto linear elíptico em que a resposta e os efeitos aleatórios têm distribuição conjunta multivariada elíptica e Osorio et al. (2007) derivaram curvaturas de influência local para essa classe de modelos mistos elípticos. Russo et al. (2009) e Russo et al. (2012) derivaram, respectivamente, procedimentos de diagnóstico e testes de hipóteses para os componentes de variância em modelos mistos não lineares elípticos, enquanto que Ibacache-Pulgar et al. (2012) derivaram procedimentos de diagnóstico em modelos mistos semiparamétricos elípticos, Manghi et al. (2016) propuseram modelos multiníveis elípticos e mais recentemente Borssoi et al. (2017) derivaram procedimentos de estimação e diagnóstico em modelos mistos lineares elípticos com uma covariável sujeita a erros de medição.

No contexto da estimação REML para modelos elípticos, Taylor e Verbyla (2006) propuseram uma aproximação tipo REML para um modelo linear t-heteroscedástico baseada no método de aproximação parcial de Laplace e na formulação de REML de Verbyla (1990). Enquanto que Vallejos e Osorio (2015) apresentaram uma extensão da estimação REML para modelos espaciais elípticos baseados na formulação de Harville (1977). Vallejos e Osorio (2015) usaram as relações entre as estimativas de máxima verossimilhança da distribuição normal multivariada e as estimativas de máxima verossimilhança de outras distribuições elípticas estudadas por Anderson et al. (1986) para estender os resultados às estimativas REML.

$\mathrm{Na}$ literatura estatística encontramos também o método de estimação denominado pseudomáxima verossimilhança proposto por Gong e Samaniego (1981) em que o parâmetro de incômodo é escalar e que foi especificada por Kano et al. (1993) e Berkane et al. (1994) para modelos elípticos. A proposta deste método é substituir um estimador dos parâmetros de incômodo na função escore dos parâmetros de interesse a fim de simplificar o procedimento de estimação. Embora o sentido deste método possa ter alguma relação como os métodos restritos, em nosso trabalho daremos ênfase a métodos relacionados com a obtenção de uma transformação na função de verossimilhança para obter uma função que dependa somente dos parâmetros da matriz de variância-covariância.

\subsection{Objetivos}

O principal objetivo deste trabalho é estudar a metodologia de estimação de máxima verossimilhança restrita em modelos lineares elípticos e modelos mistos lineares elípticos, bem como estender alguns métodos propostos para modelos lineares normais para modelos lineares elípticos. Propomos também estender a metodologia desenvolvida por Taylor e Verbyla (2006) para modelos mistos lineares t-Student. Por último, para determinados modelos, comparamos as estimativas através de estudos de simulação.

\subsection{Organização do Trabalho}

A seguir descrevemos a organização do trabalho. No Capítulo 2 apresentamos uma revisão para o modelo linear normal das metodologias de máxima verossimilhança, máxima verossimilhança restrita, máxima verossimilhança perfilada modificada e máxima verossimilhança perfilada modificada aproximada. Essas metodologias são estendidas no Capítulo 3 para os modelos lineares elípticos. Discutimos algumas estruturas usuais para a matriz de variância-covariância e particularizamos as 
equações de estimação dos modelos lineares t-Student e exponencial potência. No Capítulo 4 apresentamos a metodologia de estimação para o modelo misto linear elíptico. Uma proposta recente de estimação restrita desenvolvida para o modelo linear t-heteroscedástico é revisada no Capítulo 5 e estendida para o modelo misto linear t-Student. No Capítulo 6 algumas estruturas para a matriz de escala $\boldsymbol{\Sigma}$, apresentadas nos Capítulos 3 e 4, são particularizados para o caso t-Student e alguns estudos de simulação e aplicações são desenvolvidos. No Capítulo 7 apresentamos as conclusões do trabalho e nos apêndices resumimos algumas metodologias utilizadas no texto. 


\section{Capítulo 2}

\section{Estimação baseada na função de verossimilhança para o modelo linear normal}

Neste capítulo descrevemos alguns métodos de estimação baseados na função de verossimilhança, que são muito utilizados na literatura estatística no ajuste de modelos com distribuição normal ou pertencentes à família exponencial. Esses métodos são baseados na função de verossimilhança, no ajuste da função de verossimilhança perfilada modificada e na função de verossimilhança restrita. Os últimos dois métodos procuram diferenciar a estimação dos parâmetros de interesse dos parâmetros de incômodo. Entenda-se, parâmetros de incômodo, por aqueles parâmetros que não são relevantes na investigação, mas precisam ser estimados para especificar o modelo em estudo. De forma similar a como foi apresentada por Lee e Nelder (1996), explicaremos a metodologia considerando um modelo linear normal, faremos uma revisão dos métodos de estimação e apresentaremos os cálculos para estimar os parâmetros correspondentes.

\subsection{Modelo linear normal}

Vamos considerar inicialmente um modelo linear normal, expresso na forma

$$
\mathbf{Y}=\mathbf{X} \boldsymbol{\beta}+\boldsymbol{\epsilon},
$$

em que $\mathbf{Y}$ é um vetor de respostas $(m \times 1)$, $\mathbf{X}$ é uma matriz de planejamento $(m \times p)$ de posto coluna completo e $\boldsymbol{\epsilon}$ é um vetor aleatório com distribuição normal $m$-variada, com média $\mathbf{0}$ e matriz de variância-covariância $\boldsymbol{\Sigma}$, positiva definida. O vetor $\boldsymbol{\beta}$ de dimensão $(p \times 1)$ é um vetor de parâmetros desconhecidos e escrevemos a estrutura da matriz $\boldsymbol{\Sigma}$ como $\boldsymbol{\Sigma}(\boldsymbol{\alpha})$, em que $\boldsymbol{\alpha}$ é um vetor de parâmetros, $\boldsymbol{\alpha}=\left(\alpha_{1}, \ldots, \alpha_{j}, \ldots, \alpha_{q}\right)^{\top} \operatorname{com} q<m-p$, também desconhecidos. Assim, a função de verossimilhança para os parâmetros fica expressa na forma

$$
L(\boldsymbol{\beta}, \boldsymbol{\alpha} ; \mathbf{Y})=\operatorname{cte} *|\boldsymbol{\Sigma}|^{-1 / 2} \exp \left\{-\frac{1}{2}(\mathbf{Y}-\mathbf{X} \boldsymbol{\beta})^{\top} \boldsymbol{\Sigma}^{-1}(\mathbf{Y}-\mathbf{X} \boldsymbol{\beta})\right\}
$$

Nesta última expressão, consideramos o termo "cte"para agrupar todos os termos que não dependem dos parâmetros. O logaritmo da função de verossimilhança fica dado por

$$
l(\boldsymbol{\beta}, \boldsymbol{\alpha} ; \mathbf{Y})=\operatorname{cte}-\frac{1}{2} \log |\boldsymbol{\Sigma}|-\frac{1}{2}(\mathbf{Y}-\mathbf{X} \boldsymbol{\beta})^{\top} \boldsymbol{\Sigma}^{-1}(\mathbf{Y}-\mathbf{X} \boldsymbol{\beta}) .
$$

Na continuação, para o modelo descrito, analisaremos três métodos de estimação baseados na função de verossimilhança. 


\subsection{Estimação de máxima verossimilhança}

Mediante o método de máxima verossimilhança para encontrar os estimadores dos parâmetros desconhecidos, temos as funções escore dos parâmetros. A função escore de $\boldsymbol{\beta}$ é dada por

$$
\frac{\partial}{\partial \boldsymbol{\beta}} l(\boldsymbol{\beta}, \boldsymbol{\alpha} ; \mathbf{Y})=\mathbf{X}^{\top} \boldsymbol{\Sigma}^{-1}(\mathbf{Y}-\mathbf{X} \boldsymbol{\beta}) .
$$

A função escore de $\boldsymbol{\alpha}$ tem componentes

$$
\frac{\partial}{\partial \alpha_{j}} l(\boldsymbol{\beta}, \boldsymbol{\alpha} ; \mathbf{Y})=-\frac{1}{2} \operatorname{tr}\left\{\boldsymbol{\Sigma}^{-1} \frac{\partial \boldsymbol{\Sigma}}{\partial \alpha_{j}}\right\}+\frac{1}{2}(\mathbf{Y}-\mathbf{X} \boldsymbol{\beta})^{\top} \boldsymbol{\Sigma}^{-1} \frac{\partial \boldsymbol{\Sigma}}{\partial \alpha_{j}} \boldsymbol{\Sigma}^{-1}(\mathbf{Y}-\mathbf{X} \boldsymbol{\beta})
$$

para $j=1, \cdots, q$. Das equações de estimação baseadas nas funções escore obtemos os estimadores de máxima verossimilhança para os parâmetros. Então, o estimador de máxima verossimilhança de $\boldsymbol{\beta}$, para $\boldsymbol{\Sigma}$ conhecida, fica dado por

$$
\hat{\boldsymbol{\beta}}=\left(\mathbf{X}^{\top} \boldsymbol{\Sigma}^{-1} \mathbf{X}\right)^{-1} \mathbf{X}^{\top} \boldsymbol{\Sigma}^{-1} \mathbf{Y} .
$$

Para $\boldsymbol{\Sigma}(\boldsymbol{\alpha})$ desconhecida, temos que

$$
\hat{\boldsymbol{\beta}}_{M V}=\left(\mathbf{X}^{\top} \widehat{\boldsymbol{\Sigma}}_{M V}^{-1} \mathbf{X}\right)^{-1} \mathbf{X}^{\top} \widehat{\boldsymbol{\Sigma}}_{M V}^{-1} \mathbf{Y}
$$

Para obter o estimador da matriz de variância-covariância, vemos de (2.3) que devemos especificar uma estrutura para a matriz $\boldsymbol{\Sigma}$. Contudo, na maioria dos casos não teremos uma expressão explícita para seu estimador de máxima verossimilhança e deveremos implementar algum método de estimação. De (2.4) temos que a estrutura do estimador de $\boldsymbol{\beta}$ depende do estimador de máxima verossimilhança $\widehat{\boldsymbol{\Sigma}}_{M V}$. Para obter a matriz de informação de Fisher consideramos os resultados obtidos por Lange et al. (1989), Savalli et al. (2006) e Osorio et al. (2007). Assim, neste modelo a matriz de informação de Fisher é uma matriz diagonal por bloco dada por

$$
\mathbf{K}(\boldsymbol{\beta}, \boldsymbol{\alpha})=\left[\begin{array}{cc}
\mathbf{K}_{\beta \beta} & \mathbf{0} \\
\mathbf{0} & \mathbf{K}_{\alpha \alpha}
\end{array}\right]
$$

em que

$$
\mathbf{K}_{\beta \beta}=\mathbf{X}^{\top} \boldsymbol{\Sigma}^{-1} \mathbf{X}
$$

e o $(j, k)$-ésimo elemento de $\mathbf{K}_{\alpha \alpha}$, com $j, k$ em $1, \ldots, q$ fica dado por

$$
\mathbf{K}_{\alpha_{j} \alpha_{k}}=\frac{b_{j, k}}{4}\left(\frac{2}{m+2}-1\right)+\frac{1}{m+2} \operatorname{tr}\left\{\boldsymbol{\Sigma}^{-1} \frac{\partial \boldsymbol{\Sigma}}{\partial \alpha_{j}} \boldsymbol{\Sigma}^{-1} \frac{\partial \boldsymbol{\Sigma}}{\partial \alpha_{k}}\right\}
$$

em que $b_{j, k}$ é $\operatorname{tr}\left\{\boldsymbol{\Sigma}^{-1} \frac{\partial \boldsymbol{\Sigma}}{\partial \alpha_{j}}\right\} \operatorname{tr}\left\{\boldsymbol{\Sigma}^{-1} \frac{\partial \boldsymbol{\Sigma}}{\partial \alpha_{k}}\right\}$. Uma forma de obter as matrizes de variância e covariância assintóticas para os estimadores de máxima verossimilhança é considerar as inversas das matrizes de informação de Fisher.

\subsection{Estimação de máxima verossimilhança restrita}

A estimação de máxima verossimilhança restrita tem por objetivo separar a informação contida na função de verossimilhança em duas funções, uma que depende de todos os parâmetros desconhecidos e outra que não depende dos parâmetros de incômodo. Entenda-se por parâmetros de incômodo aqueles parâmetros que não são de interesse, mas precisam ser estimados. Este método num modelo linear, também é conhecido como técnica de contraste de erros, que consiste, essencialmente, em trabalhar com uma função de verosimilhança associada a $m-p$ contrastes dos erros linearmente independentes, entendendo por $m$ o tamanho da amostra e $p$ o número de parâmetros 
da média.

Uma divisão da informação contida na função de verossimilhança pode ser representada por uma transformação nos dados. Nisso se baseou a primeira proposta para este método de estimação, que foi apresentada por Patterson e Thompson (1971). Esses autores trabalharam com um planejamento de experimentos de blocos incompletos, cuja resposta $\mathbf{Y}$ tem distribuição normal, com vetor de médias $\mathbf{X} \boldsymbol{\beta}$, em que $\mathbf{X}$ é uma matriz de planejamento e $\boldsymbol{\beta}$ vetor de parâmetros desconhecidos de dimensão $(p \times 1)$. No entanto, a estrutura de variância-covariância, $\boldsymbol{\Sigma}=\sigma^{2} \mathbf{H}$, depende dos parâmetros $\boldsymbol{\gamma}$ e $\sigma^{2}$, também desconhecidos, isto é $\boldsymbol{\alpha}=\left(\boldsymbol{\gamma}, \sigma^{2}\right)$. A proposta é trabalhar com contrastes através da transformação da variável resposta, fazendo uma pré-multiplicação de $\mathbf{Y}$ por duas matrizes $\mathbf{S}=\mathbf{I}_{p}-\mathbf{X}\left(\mathbf{X}^{\top} \mathbf{X}\right)^{-1} \mathbf{X}^{\top}$ e $\mathbf{Q}=\mathbf{X}^{\top} \mathbf{H}$ com postos $m-p$ e $p$, respectivamente, gerando uma partição da informação de $\mathbf{Y}$ em duas variáveis independentes $\mathbf{S Y}$ e $\mathbf{Q Y}$, em que $\mathrm{E}(\mathbf{S Y})=\mathbf{0}$.

No trabalho de Harville (1974) com um enfoque de inferência Bayesiana e considerando um modelo linear da forma $\mathbf{Y}=\mathbf{X} \boldsymbol{\beta}+\boldsymbol{\epsilon}$, é discutida que a ideia de usar contrastes de erros para estimar os componentes de variância, em vez de todos os dados, é equivalente a ignorar qualquer informação a priori do vetor de parâmetros da média.

Desta forma, Harville (1974) considera vantajosa a utilização dos contrastes de erros para estimar os componentes de variância sob o enfoque Bayesiano. Do modelo linear, o vetor de respostas $\mathbf{Y}$ tem distribuição normal com vetor de médias $\boldsymbol{\beta}$ e uma estrutura de variância-covariância dependendo de um vetor de parâmetros $\boldsymbol{\alpha}$. Baseado na proposta de Patterson e Thompson (1971), Harville (1974) utiliza a mesma matriz $\mathbf{S}$, mas ao invés da matriz $\mathbf{Q}$ define uma matriz $\mathbf{G}$, de tal forma que a transformação $\mathbf{G}^{\top} \mathbf{Y}$ coincide com o estimador de máxima verossimilhança para o vetor de parâmetros $\boldsymbol{\beta}$.

Em ambas as propostas de transformação, a matriz $\mathbf{Q}$ e a matriz $\mathbf{G}$ estão em função da matriz de variância-covariância. Assim, outra transformação alternativa, porém baseada na proposta de Patterson e Thompson (1971), foi apresentada por Verbyla (1990). Para o mesmo modelo proposto por Harville (1974), Verbyla (1990) define as matrizes da transformação como sendo $\mathbf{W}_{1}$ e $\mathbf{W}_{2}$ que não dependem da estrutura de variância-covariância. Além disso, não é necessário definir uma estrutura para a matriz $\mathbf{W}_{2}$. Somente é necessário cumprir as propriedades da matriz $\mathbf{S}$ definida por Patterson e Thompson (1971).

Nesta tese daremos ênfase ao estudo da proposta de Verbyla (1990). Na próxima seção discutiremos essa transformação.

\subsubsection{Método proposto por Verbyla}

Consideremos o modelo linear normal, da equação (2.1). Supomos duas matrizes $\mathbf{W}_{1}$ e $\mathbf{W}_{2}$ de dimensão $(m \times p)$ e $(m \times(m-p))$, respectivamente, ambas de posto coluna completo e satisfazendo

$$
\mathbf{W}_{1}^{\top} \mathbf{X}=\mathbf{I}_{p} \quad e \quad \mathbf{W}_{2}^{\top} \mathbf{X}=\mathbf{0} .
$$

As colunas de $\mathbf{W}_{2}$ formam uma base do complemento ortogonal de $\mathbf{X}$. Uma forma conveniente para $\mathbf{W}_{1}^{\top}$, sugerida por Verbyla (1990) é $\left(\mathbf{X}^{\top} \mathbf{X}\right)^{-1} \mathbf{X}^{\top}$. Então, a transformação de $\mathbf{Y}$ será $\mathbf{W}^{\top} \mathbf{Y}$, tal que

$$
\mathbf{W}^{\top} \mathbf{Y}=\left[\begin{array}{c}
\mathbf{W}_{1}^{\top} \mathbf{Y} \\
\mathbf{W}_{2}^{\top} \mathbf{Y}
\end{array}\right]=\left[\begin{array}{c}
\mathbf{V}_{1} \\
\mathbf{V}_{2}
\end{array}\right]
$$

Daqui, definimos os vetores aleatórios $\mathbf{V}_{1}$ e $\mathbf{V}_{2}$ que representam a transformação particionada de $\mathbf{W}^{\top} \mathbf{Y}$. Essa partição permitirá escrever o logaritmo da função da verossimilhança como sendo a soma de duas funções, que facilitam os cálculos para encontrarmos os estimadores dos parâmetros desconhecidos.

\subsubsection{Transformação do modelo proposto}

Dado que o vetor aleatório $\mathbf{Y}$ tem distribuição normal multivariada e considerando que uma combinação linear de $\mathbf{Y}$ também terá distribuição normal, para a transformação proposta na seção 
anterior, $\mathbf{W}^{\top} \mathbf{Y}$, temos que

$$
\begin{aligned}
& \mathbf{W}^{\top} \mathbf{Y} \sim N_{m}\left(\mathbf{W}^{\top} \boldsymbol{\beta}, \mathbf{W}^{\top} \boldsymbol{\Sigma} \mathbf{W}\right) \text { ou } \\
& {\left[\begin{array}{l}
\mathbf{V}_{1} \\
\mathbf{V}_{2}
\end{array}\right] \sim N_{m}\left(\left[\begin{array}{c}
\boldsymbol{\beta} \\
\mathbf{0}
\end{array}\right],\left[\begin{array}{ll}
\mathbf{W}_{1}^{\top} \boldsymbol{\Sigma} \mathbf{W}_{1} & \mathbf{W}_{1}^{\top} \boldsymbol{\Sigma} \mathbf{W}_{2} \\
\mathbf{W}_{2}^{\top} \boldsymbol{\Sigma} \mathbf{W}_{1} & \mathbf{W}_{2}^{\top} \boldsymbol{\Sigma} \mathbf{W}_{2}
\end{array}\right]\right)}
\end{aligned}
$$

Vemos que a média da transformação não depende de $\mathbf{X}$ e para estimar $\boldsymbol{\beta}$ só precisamos da distribuição do vetor $\mathbf{V}_{1}$. Lembrando que o interesse é dividir a função de verossimilhança em duas funções. Podemos escrever a função de verossimilhança da transformação como sendo o produto da função de verossimilhança condicional de $\mathbf{V}_{1}$ dado $\mathbf{V}_{2}$ e a função de verossimilhança marginal de $\mathbf{V}_{2}$. Isto é,

$$
L\left(\boldsymbol{\beta}, \boldsymbol{\Sigma} ; \mathbf{V}_{1}, \mathbf{V}_{2}\right)=L\left(\boldsymbol{\beta}, \boldsymbol{\Sigma} ; \mathbf{V}_{1} \mid \mathbf{V}_{2}\right) L\left(\boldsymbol{\Sigma} ; \mathbf{V}_{2}\right) .
$$

Aplicando logaritmo, obtemos

$$
l\left(\boldsymbol{\beta}, \boldsymbol{\Sigma} ; \mathbf{V}_{1}, \mathbf{V}_{2}\right)=l\left(\boldsymbol{\beta}, \boldsymbol{\Sigma} ; \mathbf{V}_{1} \mid \mathbf{V}_{2}\right)+l\left(\boldsymbol{\Sigma} ; \mathbf{V}_{2}\right) .
$$

Em virtude da suposição de normalidade para a variável $\mathbf{Y}$, as distribuições marginais e condicionais também têm distribuição normal. Portanto, a distribuição marginal de $\mathbf{V}_{2}$ fica dada por

$$
\mathbf{W}_{2}^{\top} \mathbf{Y}=\mathbf{V}_{2} \sim N_{m-p}\left(\mathbf{0}, \mathbf{W}_{2}^{\top} \boldsymbol{\Sigma} \mathbf{W}_{2}\right) .
$$

Sabemos que a média de uma distribuição condicional normal pode ser escrita como

$$
\boldsymbol{\mu}_{1}\left(\mathbf{v}_{2}\right)=\boldsymbol{\mu}_{1}+\operatorname{Cov}\left(\mathbf{V}_{1}, \mathbf{V}_{2}\right) \operatorname{Var}\left(\mathbf{V}_{2}\right)^{-1}\left(\mathbf{v}_{2}-\boldsymbol{\mu}_{2}\right),
$$

em que $\mathbf{v}_{2}$ representa um valor da variável $\mathbf{V}_{2}$. Enquanto que, $\boldsymbol{\mu}_{1}$ e $\boldsymbol{\mu}_{2}$ representam as médias de $\mathbf{V}_{1}$ e $\mathbf{V}_{2}$, respectivamente. Na transformação (2.5), $\boldsymbol{\mu}_{1}=\boldsymbol{\beta}$ e $\boldsymbol{\mu}_{2}=\mathbf{0}$, então

$$
\boldsymbol{\mu}_{1}\left(\mathbf{v}_{2}\right)=\boldsymbol{\beta}+\mathbf{W}_{1}^{\top} \boldsymbol{\Sigma} \mathbf{W}_{2}\left(\mathbf{W}_{2}^{\top} \boldsymbol{\Sigma} \mathbf{W}_{2}\right)^{-1} \mathbf{W}_{2} \mathbf{Y}=\boldsymbol{\beta}+\mathbf{C}_{W} .
$$

De forma conveniente, escrevemos a esperança condicional como a soma de dois componentes, em que o segundo componente, $\mathbf{C}_{W}$, não depende do vetor de parâmetros $\boldsymbol{\beta}$. Como acima, a equação (A.5) do Apêndice A é a matriz de variância-covariância de uma distribuição condicional da transformação. Então, temos que

$$
\boldsymbol{\Sigma}_{1}\left(\mathbf{v}_{2}\right)=\mathbf{W}_{1}^{\top} \mathbf{X}\left(\mathbf{X}^{\top} \boldsymbol{\Sigma}^{-1} \mathbf{X}\right)^{-1} \mathbf{X}^{\top} \mathbf{W}_{1} .
$$

Portanto, a distribuição condicional de $\mathbf{V}_{1}$ dado $\mathbf{V}_{2}$ fica expressa na forma

$$
\mathbf{V}_{1} \mid \mathbf{V}_{2} \sim N_{p}\left(\boldsymbol{\beta}+\mathbf{C}_{W}, \mathbf{W}_{1}^{\top} \mathbf{X}\left(\mathbf{X}^{\top} \boldsymbol{\Sigma}^{-1} \mathbf{X}\right)^{-1} \mathbf{X}^{\top} \mathbf{W}_{1}\right)
$$

A proposta de Verbyla (1990) é estimar o vetor de parâmetros $\boldsymbol{\beta}$ através da função de verossimilhança da distribuição condicional e os componentes da matriz de parâmetros $\boldsymbol{\Sigma}$ considerando a função de verossimilhança da distribuição marginal de $\mathbf{V}_{2}$. Logo, usando os conceitos preliminares, escreveremos os logaritmos das funções de verossimilhança de forma conveniente para facilitar os cálculos das estimativas. A primeira função do lado direito de (2.6), isto é a função de verossimilhança condicional de $\mathbf{V}_{1}$ dado $\mathbf{V}_{2}$, fica dada por

$$
l\left(\boldsymbol{\beta}, \boldsymbol{\Sigma} ; \mathbf{V}_{1} \mid \mathbf{V}_{2}\right)=\operatorname{cte}-\frac{1}{2}\left(\mathbf{V}_{1}-\boldsymbol{\beta}-\mathbf{C}_{W}\right)^{\top}\left\{\mathbf{W}_{1}^{\top} \mathbf{X}\left(\mathbf{X}^{\top} \boldsymbol{\Sigma}^{-1} \mathbf{X}\right)^{-1} \mathbf{X}^{\top} \mathbf{W}_{1}\right\}^{-1}\left(\mathbf{V}_{1}-\boldsymbol{\beta}-\mathbf{C}_{W}\right) .
$$

Nesta última expressão, o termo "cte"para agrupar todos os termos que não dependem de $\boldsymbol{\beta}$. Usando a decomposição do determinante apresentada na equação (A.8) e o resultado do lema, dado pela 
equação (A.4), podemos escrever o logaritmo da função de verossimilhança marginal de $\mathbf{V}_{2}$ como

$$
\begin{aligned}
l\left(\boldsymbol{\Sigma} ; \mathbf{V}_{2}\right)= & \text { cte }-\frac{1}{2} \log \left|\mathbf{X}^{\top} \boldsymbol{\Sigma}^{-1} \mathbf{X}\right|-\frac{1}{2} \log |\boldsymbol{\Sigma}| \\
& -\frac{1}{2} \mathbf{Y}^{\top}\left\{\boldsymbol{\Sigma}^{-1}-\boldsymbol{\Sigma}^{-1} \mathbf{X}\left(\mathbf{X}^{\top} \boldsymbol{\Sigma}^{-1} \mathbf{X}\right)^{-1} \mathbf{X}^{\top} \boldsymbol{\Sigma}^{-1}\right\} \mathbf{Y}
\end{aligned}
$$

Por último, usando a notação $\mathbf{b}=\left(\mathbf{X}^{\top} \boldsymbol{\Sigma}^{-1} \mathbf{X}\right)^{-1} \mathbf{X}^{\top} \boldsymbol{\Sigma}^{-1} \mathbf{Y}$ e como $\mathbf{b}^{\top} \mathbf{X}^{\top} \boldsymbol{\Sigma}^{-1} \mathbf{X} \mathbf{b}=\mathbf{Y}^{\top} \boldsymbol{\Sigma}^{-1} \mathbf{X b}$, temos que o logaritmo da função de verossimilhança de $\mathbf{V}_{2}$ fica dado por

$$
\begin{aligned}
l\left(\boldsymbol{\Sigma} ; \mathbf{V}_{2}\right)= & \text { cte }-\frac{1}{2} \log \left|\mathbf{X}^{\top} \boldsymbol{\Sigma}^{-1} \mathbf{X}\right|-\frac{1}{2} \log |\boldsymbol{\Sigma}| \\
& -\frac{1}{2}(\mathbf{Y}-\mathbf{X b})^{\top} \boldsymbol{\Sigma}^{-1}(\mathbf{Y}-\mathbf{X b}) .
\end{aligned}
$$

Vemos que $\mathbf{b}$ tem a mesma estrutura do estimador de MV de $\boldsymbol{\beta}$, mas utilizaremos $\mathbf{b}$ para diferenciar os métodos de estimação dado que esses métodos dependem da estimação dos parâmetros da matriz de variância-covariância que serão estimados de formas diferentes.

\subsubsection{Derivação dos estimadores de máxima verossimilhança restrita}

Considerando a decomposição do logaritmo da função de verossimilhança da transformação proposto por Verbyla (1990) e do método de estimação proposto por Patterson e Thompson (1971), podemos obter o estimador de máxima verossimilhança restrita para $\boldsymbol{\beta}$ a partir do logaritmo da função de verossimilhança condicional de $\mathbf{V}_{1} \mid \mathbf{V}_{2}$. Lembrando que $\mathbf{W}_{1}^{\top}=\left(\mathbf{X}^{\top} \mathbf{X}\right)^{-1} \mathbf{X}^{\top}$ e os cálculos apresentados no Apêndice B, equação (B.4), a função escore com respeito a $\boldsymbol{\beta}$, fica dada por

$$
\frac{\partial l\left(\boldsymbol{\beta}, \boldsymbol{\Sigma} ; \mathbf{V}_{1} \mid \mathbf{V}_{2}\right)}{\partial \boldsymbol{\beta}}=\left(\mathbf{X}^{\top} \boldsymbol{\Sigma}^{-1} \mathbf{X}\right)(\mathbf{b}-\boldsymbol{\beta})
$$

Finalmente, igualando a zero, obtemos

$$
\hat{\boldsymbol{\beta}}=\left(\mathbf{X}^{\top} \boldsymbol{\Sigma}^{-1} \mathbf{X}\right)^{-1} \mathbf{X}^{\top} \boldsymbol{\Sigma}^{-1} \mathbf{Y}
$$

A forma do estimador é a mesma do estimador de máxima verossimilhança de $\boldsymbol{\beta}$ obtido em (2.4), mas depende de $\boldsymbol{\Sigma}$. Então, como já foi mencionado, para obter o estimador de $\boldsymbol{\Sigma}$ devemos considerar a função de verossimilhança marginal de $\mathbf{V}_{2}$. Assim, para o elemento $\alpha_{j}$ com $j=1, \ldots, q$, que corresponde ao $j$-ésimo elemento do vetor $\boldsymbol{\alpha}$, temos que a derivada de $l\left(\boldsymbol{\Sigma} ; \mathbf{V}_{2}\right)$ com respeito a $\alpha_{j}$ fica dada por

$$
\begin{aligned}
\frac{\partial l\left(\boldsymbol{\Sigma} ; \mathbf{V}_{2}\right)}{\partial \alpha_{j}}= & \frac{1}{2} \operatorname{tr}\left\{\left(\mathbf{X}^{\top} \boldsymbol{\Sigma}^{-1} \mathbf{X}\right)^{-1} \mathbf{X}^{\top} \boldsymbol{\Sigma}^{-1} \frac{\partial \boldsymbol{\Sigma}}{\partial \alpha_{j}} \boldsymbol{\Sigma}^{-1} \mathbf{X}\right\}-\frac{1}{2} \operatorname{tr}\left\{\boldsymbol{\Sigma}^{-1} \frac{\partial \boldsymbol{\Sigma}}{\partial \alpha_{j}}\right\} \\
& +\frac{1}{2}(\mathbf{Y}-\mathbf{X} \mathbf{b})^{\top} \boldsymbol{\Sigma}^{-1} \frac{\partial \boldsymbol{\Sigma}}{\partial \alpha_{j}} \boldsymbol{\Sigma}^{-1}(\mathbf{Y}-\mathbf{X b})
\end{aligned}
$$

para $j=1, \ldots, q$. O detalhe do último termo deste resultado é apresentado na equação (B.7) do Apêndice B. Para encontrar o estimador da matriz de variância-covariância devemos definir uma estrutura para a matriz, mas na maioria dos casos os estimadores não terão forma explícita, e serão calculados através de algum método de aproximação. O estimador resultante será denominado estimador de máxima verossimilhança restrita de $\boldsymbol{\Sigma}$, que escreveremos como $\widehat{\boldsymbol{\Sigma}}_{M V R}$. Ao substituir $\boldsymbol{\Sigma}$ por $\widehat{\boldsymbol{\Sigma}}_{M V R}$ na expressão (2.8), obtemos o estimador de máxima verossimilhança restrita de $\boldsymbol{\beta}$, dado por

$$
\hat{\boldsymbol{\beta}}_{M V R}=\left(\mathbf{X}^{\top} \widehat{\boldsymbol{\Sigma}}_{M V R}^{-1} \mathbf{X}\right)^{-1} \mathbf{X}^{\top} \widehat{\boldsymbol{\Sigma}}_{M V R}^{-1} \mathbf{Y}
$$


O enfoque de Harville (1974) com respeito à equação (2.6) é obter uma função de verossimilhança marginal ou condicional dos parâmetros de interesse. Na próxima seção explicaremos a metodologia da função de verossimilhança perfilada modificada e sua relação com o enfoque de Harville (1974).

\subsection{Estimação usando a função de verossimilhança perfilada modi- ficada}

Consideramos a parametrização do modelo em dois grupos de parâmetros, isto é, $\boldsymbol{\alpha}$ denominado de interesse e $\boldsymbol{\beta}$ parâmetro de incômodo. A função de verossimilhança marginal é a construção de uma função de verossimilhança baseada numa estatística $T$, tal que sua distribuição dependa apenas dos parâmetros de interesse. Ou seja, que a função de verossimilhança condicional surge da distribuição condicional dos dados dada uma estatística $S, Y \mid S=s$, que dependa somente dos parâmetros de interesse. Em ambos os casos teremos uma função de verossimilhança genuína mas que pode não considerar toda a informação de $\boldsymbol{\alpha}$ contida na amostra, assim nem sempre será fácil determinar as estatísticas $T$ ou $S$.

Então, surge a proposta de Barndorff-Nielsen (1983), um método de estimação baseado numa modificação da função de verossimilhança perfilada para estimar um vetor de parâmetros de interesse, $\boldsymbol{\alpha}$, dado que os demais parâmetros estão fixados, $\boldsymbol{\beta}$. A função resultante é denominada função de verosimilhança perfilada modificada e é baseada na distribuição assintótica dos estimadores de máxima verossimilhança dos parâmetros fixados. Desta forma, não precisamos conhecer a distribuição exata dos estimadores e o método pode ser aplicado em qualquer problema de estimação. Cox e Reid (1987) propuseram um ajuste da verossimilhança perfilada modificada analisando um caso particular com o parâmetro $\alpha$ como escalar. Nesse trabalho a ortogonalidade dos parâmetros é um fato importante, pois se existe ortogonalidade a função de verossimilhança perfilada modificada proposta por Barndorff-Nielsen (1983) é simplificada.

No livro de Lee et al. (2006), num ajuste da verossimilhança perfilada modificada, mostra-se um exemplo da distribuição normal multivariada mencionando que o resultado encontrado coincide com a expressão obtida para o método de estimação de máxima verossimilhança restrita, proposta por Patterson e Thompson (1971) apresentado na seção anterior.

Em seguida, resumimos os resultados e analisamos o caso da distribuição normal. Porém, precisamos de alguns conceitos para explicar a metodologia que são apresentamos no Apêndice A.

\subsubsection{Derivação do estimador de máxima verossimilhança perfilada modificada}

Da proposta de Barndorff-Nielsen (1983), considerando a parametrização do modelo com dois grupos de parâmetros: $\boldsymbol{\alpha}$ denominado de interesse e $\boldsymbol{\beta}$ parâmetro de incômodo, temos que o logaritmo da função de verossimilhança perfilada modificada de $\boldsymbol{\alpha}$ é definido como

$$
l_{p m}(\boldsymbol{\alpha})=l_{p}(\boldsymbol{\alpha})-\frac{1}{2} \log \left\{\left|\mathbf{I}_{\beta \beta}\left(\hat{\boldsymbol{\beta}}_{\alpha}, \boldsymbol{\alpha}\right)\right|\right\}+\log \left|\frac{\partial \hat{\boldsymbol{\beta}}}{\partial \hat{\boldsymbol{\beta}}_{\alpha}}\right|,
$$

em que $l_{p}(\boldsymbol{\alpha})$ é a função de verossimilhança perfilada de $\boldsymbol{\alpha}$, isto é, o logaritmo da função de verossimilhança completa avaliada no estimador de máxima verossimilhança parcial de $\boldsymbol{\beta}$, denotado por $\hat{\boldsymbol{\beta}}_{\alpha}$. Entendemos o estimador de máxima verossimilhança parcial de $\boldsymbol{\beta}$ como sendo o estimador de máxima verossimilhança de $\boldsymbol{\beta}$ com o vetor $\boldsymbol{\alpha}$ fixado. O termo $\mathbf{I}_{\beta \beta}\left(\hat{\boldsymbol{\beta}}_{\alpha}, \boldsymbol{\alpha}\right)$ denota a matriz de informação observada de $\boldsymbol{\beta}$ avaliada em $\hat{\boldsymbol{\beta}}_{\alpha}$ e $\left|\frac{\partial \hat{\boldsymbol{\beta}}}{\partial \hat{\boldsymbol{\beta}}_{\alpha}}\right|$ representa o Jacobiano entre o estimador de máxima verossimilhança $\hat{\boldsymbol{\beta}}$ e o estimador de máxima verossimilhança parcial $\hat{\boldsymbol{\beta}}_{\alpha}$ para um valor de $\alpha$ fixo. Em Severini (2000)Cap. 9) são apresentadas diferentes ajustes e aproximações para a função de verossimilhança perfilada modificada. Neste trabalho consideramos a função de verossimilhança perfilada modificada ajustada, proposta por Cox e Reid (1987). Se existe a ortogonalidade entre os parâmetros, a função de verossimilhança perfilada modificada ajustada pode ser definida, $l_{p m}^{*}(\boldsymbol{\alpha})$, 
como

$$
l_{p m}^{*}(\boldsymbol{\alpha})=l_{p}(\boldsymbol{\alpha})-\frac{1}{2} \log \left\{\left|\mathbf{I}_{\beta \beta}\left(\hat{\boldsymbol{\beta}}_{\alpha}, \boldsymbol{\alpha}\right)\right|\right\} .
$$

Vemos que esta última expressão (2.10) não depende do parâmetro $\boldsymbol{\beta}$ e portanto $l_{p m}^{*}(\boldsymbol{\alpha})$ pode ser considerada para estimar os parâmetros do vetor $\boldsymbol{\alpha}$. O estimador de máxima verossimilhança parcial do vetor $\boldsymbol{\beta}$ pode ser obtido da função de verossimilhança completa (em (2.2)) ou podemos maximizar a seguinte função:

$$
l^{*}(\boldsymbol{\beta}, \boldsymbol{\alpha})=l(\boldsymbol{\beta}, \boldsymbol{\alpha} ; \mathbf{Y})-\frac{1}{2} \log \left\{\left|\mathbf{I}_{\beta \beta}\left(\hat{\boldsymbol{\beta}}_{\alpha}, \boldsymbol{\alpha}\right)\right|\right\} .
$$

Desta forma, temos que o estimador de máxima verossimilhança parcial de $\boldsymbol{\beta}$ terá a mesma estrutura do estimador de máxima verossimilhança $\left(\hat{\boldsymbol{\beta}}_{M V}\right)$. Agora, considerando o caso particular do modelo linear normal, a matriz de informação observada de $\boldsymbol{\beta}$ avaliada em $\hat{\boldsymbol{\beta}}_{\alpha}$ é dada por $\mathbf{X}^{\top} \boldsymbol{\Sigma}^{-1} \mathbf{X}$. Assim, para este caso particular, o logaritmo da função de verossimilhança perfilada modificada ajustada para $\boldsymbol{\alpha}$, obtida de (2.10), fica dado por

$$
l_{p m}^{*}(\boldsymbol{\alpha})=-\frac{1}{2} \log |\boldsymbol{\Sigma}|-\frac{1}{2}\left(\mathbf{Y}-\mathbf{X} \hat{\boldsymbol{\beta}}_{\alpha}\right)^{\top} \boldsymbol{\Sigma}^{-1}\left(\mathbf{Y}-\mathbf{X} \hat{\boldsymbol{\beta}}_{\alpha}\right)-\frac{1}{2} \log \left\{\left|\mathbf{X}^{\top} \boldsymbol{\Sigma}^{-1} \mathbf{X}\right|\right\} .
$$

Podemos considerar esta última expressão como uma pseudo-verossimilhança do vetor $\boldsymbol{\alpha}$, isto é, uma função dos dados que depende apenas dos parâmetros de interesse e que não necessariamente é uma função de verossimilhança genuína. Derivando $l_{p m}^{*}(\boldsymbol{\alpha})$ com respeito a $\boldsymbol{\alpha}$ podemos encontrar um estimador para a estrutura de $\boldsymbol{\Sigma}$

$$
\begin{aligned}
\frac{\partial}{\partial \alpha_{j}} l_{p m}^{*}(\boldsymbol{\alpha})= & -\frac{1}{2} \operatorname{tr}\left\{\boldsymbol{\Sigma}^{-1} \frac{\partial \boldsymbol{\Sigma}}{\partial \alpha_{j}}\right\}+\frac{1}{2}\left(\mathbf{Y}-\mathbf{X} \hat{\boldsymbol{\beta}}_{\alpha}\right)^{\top} \boldsymbol{\Sigma}^{-1} \frac{\partial \boldsymbol{\Sigma}}{\partial \alpha_{j}} \boldsymbol{\Sigma}^{-1}\left(\mathbf{Y}-\mathbf{X} \hat{\boldsymbol{\beta}}_{\alpha}\right) \\
& +\frac{1}{2} \operatorname{tr}\left\{\left(\mathbf{X}^{\top} \boldsymbol{\Sigma}^{-1} \mathbf{X}\right)^{-1} \mathbf{X}^{\top} \boldsymbol{\Sigma}^{-1} \frac{\partial \boldsymbol{\Sigma}}{\partial \alpha_{j}} \boldsymbol{\Sigma}^{-1} \mathbf{X}\right\}
\end{aligned}
$$

para $j=1, \ldots, q$. A última igualdade é válida considerando a equação (B.6) do Apêndice B. Para obter o estimador da matriz $\boldsymbol{\Sigma}$ devemos definir uma estrutura para a matriz, mas na maioria dos casos não é possível obter um estimador explícito para a estrutura de $\boldsymbol{\Sigma}$. Utilizando algum método de aproximação podemos encontrar uma expressão para o estimador de máxima verossimilhança perfilada modificada ajustada para $\boldsymbol{\Sigma}$, que denotamos por $\widehat{\boldsymbol{\Sigma}}_{M V P M}$. Portanto, obtemos

$$
\hat{\boldsymbol{\beta}}_{M V P M}=\left(\mathbf{X}^{\top} \widehat{\boldsymbol{\Sigma}}_{M V P M}^{-1} \mathbf{X}\right)^{-1} \mathbf{X}^{\top} \widehat{\boldsymbol{\Sigma}}_{M V P M}^{-1} \mathbf{Y} .
$$

Na próxima seção apresentamos um caso particular de estrutura para a matriz de variânciacovariância para obter estimativas explícitas. Consideraremos um modelo linear normal homocedástico, a fim de ilustrar a importância em trabalhar com métodos alternativos ao método de máxima verossimilhança tradicional.

\subsection{Caso particular da matriz de variância-covariância}

Nesta seção consideramos um caso particular do modelo apresentado em (2.1). Aqui, a estrutura para a matriz $\boldsymbol{\Sigma}$ será reduzida a $\sigma^{2} \mathbf{I}_{m}$. Isto é, além de normalidade, assumimos independência entre os componentes de $\mathbf{Y}$ e homogeneidade das variâncias. Em seguida, obtemos os estimadores dos parâmetros, substituindo $\boldsymbol{\Sigma}=\sigma^{2} \mathbf{I}_{m}$, nas expressões derivadas para cada método de estimação descritos nas seções anteriores. De outra forma, note que $\boldsymbol{\alpha}=\sigma^{2}$, um escalar. O estimador de $\boldsymbol{\beta}$, 
como já vimos, tem a mesma estrutura para os três métodos de estimação, sendo dado por

$$
\begin{aligned}
\hat{\boldsymbol{\beta}} & =\left(\mathbf{X}^{\top} \boldsymbol{\Sigma}^{-1} \mathbf{X}\right)^{-1} \mathbf{X}^{\top} \boldsymbol{\Sigma}^{-1} \mathbf{Y} \\
& =\left\{\mathbf{X}^{\top}\left(\sigma^{2} \mathbf{I}_{m}\right)^{-1} \mathbf{X}\right\}^{-1} \mathbf{X}^{\top}\left(\sigma^{2} \mathbf{I}_{m}\right)^{-1} \mathbf{Y} \\
& =\left(\mathbf{X}^{\top} \mathbf{X}\right)^{-1} \mathbf{X}^{\top} \mathbf{Y} .
\end{aligned}
$$

Como podemos notar, nessa última expressão, o estimador não depende de $\sigma^{2}$, portanto, para os três métodos de estimação, teremos a mesma estimativa. Para o estimador de máxima verossimilhança de $\sigma^{2}$, consideramos os resultados da Seção 2.2. A função escore para este caso fica dada por

$$
\begin{aligned}
\frac{\partial}{\partial \sigma^{2}} l\left(\boldsymbol{\beta}, \sigma^{2}\right) & =-\frac{1}{2} \frac{\partial}{\partial \sigma^{2}} \log \left|\sigma^{2} \mathbf{I}_{m}\right|-\frac{1}{2} \frac{\partial}{\partial \sigma^{2}}(\mathbf{Y}-\mathbf{X} \boldsymbol{\beta})^{\top}\left(\sigma^{2} \mathbf{I}_{m}\right)^{-1}(\mathbf{Y}-\mathbf{X} \boldsymbol{\beta}) \\
& =-\frac{1}{2 \sigma^{2}} \operatorname{tr}\left\{\mathbf{I}_{m}\right\}+\frac{1}{2 \sigma^{4}}(\mathbf{Y}-\mathbf{X} \boldsymbol{\beta})^{\top}(\mathbf{Y}-\mathbf{X} \boldsymbol{\beta}) .
\end{aligned}
$$

Portanto, a equação de estimação fica dada por,

$$
-\frac{1}{2 \sigma^{2}} m+\frac{1}{2 \sigma^{4}}(\mathbf{Y}-\mathbf{X} \boldsymbol{\beta})^{\top}(\mathbf{Y}-\mathbf{X} \boldsymbol{\beta})=0 .
$$

Assim, obtemos que o estimador de máxima verossimilhança assume a forma

$$
\hat{\sigma}_{M V}^{2}=\frac{1}{m}(\mathbf{Y}-\mathbf{X} \hat{\boldsymbol{\beta}})^{\top}(\mathbf{Y}-\mathbf{X} \hat{\boldsymbol{\beta}}),
$$

cuja esperança é

$$
\mathrm{E}\left(\hat{\sigma}_{M V}^{2}\right)=\frac{(m-p)}{m} \sigma^{2} .
$$

Vemos que $\hat{\sigma}_{M V}^{2}$ é um estimador viesado de $\sigma^{2}$. A seguir derivamos os estimadores de máxima verossimilhança restrita e o ajuste da perfilada modificada para $\sigma^{2}$. Anteriormente, vimos que as equações de estimação obtidas em ambos os casos coincidem. Então, consideraremos a equação (2.9) com $\boldsymbol{\Sigma}=\sigma^{2} \mathbf{I}_{m}$, obtendo

$$
\begin{aligned}
\frac{\partial l\left(\boldsymbol{\Sigma} ; \mathbf{V}_{2}\right)}{\partial \sigma^{2}}= & \frac{1}{2} \operatorname{tr}\left\{\left(\mathbf{X}^{\top} \boldsymbol{\Sigma}^{-1} \mathbf{X}\right)^{-1} \mathbf{X}^{\top} \boldsymbol{\Sigma}^{-1} \frac{\partial \boldsymbol{\Sigma}}{\partial \sigma^{2}} \boldsymbol{\Sigma}^{-1} \mathbf{X}\right\}-\frac{1}{2} \operatorname{tr}\left\{\boldsymbol{\Sigma}^{-1} \frac{\partial \boldsymbol{\Sigma}}{\partial \sigma^{2}}\right\} \\
& +\frac{1}{2}(\mathbf{Y}-\mathbf{X} \mathbf{b})^{\top} \boldsymbol{\Sigma}^{-1} \frac{\partial \boldsymbol{\Sigma}}{\partial \sigma^{2}} \boldsymbol{\Sigma}^{-1}(\mathbf{Y}-\mathbf{X} \mathbf{b}) \\
= & \frac{1}{2 \sigma^{2}} \operatorname{tr}\left\{\left(\mathbf{X}^{\top} \mathbf{X}\right)^{-1} \mathbf{X}^{\top} \mathbf{X}\right\}-\frac{1}{2 \sigma^{2}} \operatorname{tr}\left\{\mathbf{I}_{m}\right\}+\frac{1}{2 \sigma^{4}}(\mathbf{Y}-\mathbf{X b})^{\top}(\mathbf{Y}-\mathbf{X} \mathbf{b}) \\
= & -\frac{m-p}{2 \sigma^{2}}+\frac{1}{2 \sigma^{4}}(\mathbf{Y}-\mathbf{X} \mathbf{b})^{\top}(\mathbf{Y}-\mathbf{X b}) .
\end{aligned}
$$

Da equação de estimação resultante temos que o estimador para $\sigma^{2}$ fica dada por

$$
\hat{\sigma}_{M V P M}^{2}=\hat{\sigma}_{M V R}^{2}=\frac{1}{m-p}(\mathbf{Y}-\mathbf{X} \hat{\boldsymbol{\beta}})^{\top}(\mathbf{Y}-\mathbf{X} \hat{\boldsymbol{\beta}})=\frac{m}{m-p} \hat{\sigma}_{M V}^{2}
$$

cuja esperança é dada por

$$
\mathrm{E}\left(\hat{\sigma}_{M V R}^{2}\right)=\sigma^{2}
$$

Desta forma, vemos que através dos métodos de estimação perfilada modificada e restrita obtemos um estimador não viesado para $\sigma^{2}$. Já pelo método de estimação de máxima verossimilhança o estimador tradicional de $\sigma^{2}$ é viesado. 


\subsection{Comentários}

Nas últimas seções, explicamos e derivamos os estimadores e as equações de estimação para os parâmetros $\boldsymbol{\beta}$ e $\boldsymbol{\Sigma}$ de um modelo linear normal. Trabalhamos com três métodos de estimação: o primeiro foi o método clássico de maximização da função de verossimilhança e os dois métodos seguintes são modificações da função de verossimilhança que têm como objetivo estimar os componentes de variância de uma forma mais direta, em que as estimativas resultantes consideram a perda de graus de liberdade pela estimação do vetor de médias ou parâmetros de incômodo.

Para os três métodos encontramos a mesma estrutura explícita para os estimadores de $\boldsymbol{\beta}$. Os estimadores têm a mesma estrutura nos três métodos, mas dependem da estimativa de $\boldsymbol{\Sigma}$, cuja estimação não é explícita em nenhum dos métodos de estimação.

Ao compararmos as equações de estimação de $\boldsymbol{\Sigma}$, vemos que os métodos de estimação de máxima verossimilhança perfilada modificada (2.11) e restrita (2.9) levam aos mesmos resultados, considerando que $\mathbf{b}=\left(\mathbf{X}^{\top} \boldsymbol{\Sigma}^{-1} \mathbf{X}\right)^{-1} \mathbf{X}^{\top} \boldsymbol{\Sigma}^{-1} \mathbf{Y}=\hat{\boldsymbol{\beta}}_{\alpha}$. Essas igualdades nos resultados para as funções de verossimilhança resultantes para o caso da distribuição normal foram também obtidas por Verbeke e Molenberghs (2000) e Lee et al. (2006). Entretanto, a equação de estimação de $\boldsymbol{\Sigma}$ pelo método de máxima verossimilhança (2.3) tem um termo a menos.

As propriedades assintóticas dos estimadores de máxima verossimilhança restritas num modelo misto linear normal são consistente e assintoticamente normal (Ver Pinheiro e Bates (2000)). Portanto, uma aproximação aos erros padrão dos estimadores de máxima verossimilhança restrita é menos a matriz de informação de Fisher. Mas nas aplicações apresentadas no Capítulo 6 consideramos uma estimativa dos erros padrões utilizando um procedimento bootstrap não paramétrico.

Um dos objetivo deste trabalho é estudar a aplicabilidade dos métodos derivados da função de verossimilhança e comparar as estimativas para modelos com distribuição elíptica. Portanto, é importante mostrar o procedimento e suas semelhanças como o caso normal. Isso será discutido no próximo capítulo. 
14 ESTIMAÇÃO BASEADA NA FUNÇÃO DE VEROSSIMILHANÇA PARA O MODELO LINEAR NORMAL 


\section{Capítulo 3}

\section{Métodos de estimação no modelo linear elíptico}

Neste capítulo discutimos a aplicabilidade dos métodos de estimação baseados na função de verossimilhança, vistos no capítulo anterior, para a família de distribuições elípticas. Precisaremos de algumas propriedades das distribuições elípticas que são apresentadas no Apêndice C, baseados em Fang et al. (1990) e na Tese de Doutorado de Arellano (1994). Por último, exemplificaremos alguns casos particulares de distribuições que pertencem à família elíptica.

\subsection{Modelo linear elíptico}

Consideramos a formulação do modelo linear elíptico estudado na Tese de Doutorado de Arellano (1994), temos que

$$
\mathbf{Y}=\mathbf{X} \boldsymbol{\beta}+\boldsymbol{\epsilon}
$$

em que $\mathbf{Y}$ é um vetor de respostas $(m \times 1)$, $\mathbf{X}$ é uma matriz de planejamento $(m \times p)$ de posto coluna completo e $\boldsymbol{\epsilon}$ é um vetor aleatório com média $\mathbf{0}$ e matriz de escala $\boldsymbol{\Sigma}$ positiva definida. O vetor $\boldsymbol{\beta}$ de dimensão $(p \times 1)$ é um vetor de parâmetros desconhecidos e escrevemos a estrutura da matriz $\boldsymbol{\Sigma}$ como $\boldsymbol{\Sigma}(\boldsymbol{\alpha})$, em que $\boldsymbol{\alpha}$ é um vetor de parâmetros, $\boldsymbol{\alpha}=\left(\alpha_{1}, \ldots, \alpha_{j}, \ldots, \alpha_{q}\right)^{\top} \operatorname{com} q<m-p$, também desconhecidos. $\mathrm{O}$ vetor de respostas $\mathbf{Y}$ tem distribuição elíptica de parâmetros $\boldsymbol{\mu}_{(m \times 1)}$ e $\boldsymbol{\Sigma}_{(m \times m)}$ que denotaremos como

$$
\mathbf{Y} \sim E l_{m}(\boldsymbol{\mu}, \boldsymbol{\Sigma}, g)
$$

em que $g: \mathbb{R} \rightarrow[0, \infty)$ e satisfaz a condição que $\int_{0}^{\infty} u^{m / 2-1} g(u) d u<\infty$, chamada função geradora de densidade, pois determina a função de densidade conjunta de $\mathbf{Y}$, que é dada por

$$
f_{\mathbf{Y}}(\mathbf{y}, \boldsymbol{\mu}, \boldsymbol{\Sigma})=|\boldsymbol{\Sigma}|^{-1 / 2} g(u),
$$

em que $u$ é a forma quadrática $(\mathbf{Y}-\boldsymbol{\mu})^{\top} \boldsymbol{\Sigma}^{-1}(\mathbf{Y}-\boldsymbol{\mu})$. Consideramos a seguinte estrutura para os parâmetros:

$$
\boldsymbol{\mu}=\mathbf{X} \boldsymbol{\beta} \quad \text { e } \quad \boldsymbol{\Sigma}=\boldsymbol{\Sigma}(\boldsymbol{\alpha}) .
$$

Portanto, a função de verossimilhança para o modelo linear elíptico é dada por

$$
L(\boldsymbol{\beta}, \boldsymbol{\alpha} ; \mathbf{Y})=|\boldsymbol{\Sigma}|^{-1 / 2} g(u),
$$

e o logaritmo da função de verossimilhança fica expresso na forma

$$
l(\boldsymbol{\beta}, \boldsymbol{\alpha} ; \mathbf{Y})=-\frac{1}{2} \log |\boldsymbol{\Sigma}|+\log g(u)=-\frac{1}{2} \log |\boldsymbol{\Sigma}|+\log g\left\{(\mathbf{Y}-\mathbf{X} \boldsymbol{\beta})^{\top} \boldsymbol{\Sigma}^{-1}(\mathbf{Y}-\mathbf{X} \boldsymbol{\beta})\right\},
$$


lembrando que por (3.1), a matriz $\boldsymbol{\Sigma}$ depende do vetor de parâmetros $\boldsymbol{\alpha}$. Na Tabela 3.1 apresentamos algumas distribuições pertencentes à família elíptica e portanto temos algumas formas que assume a função $g(\cdot)$.

Tabela 3.1: Resumo de algumas distribuições pertencentes à família elíptica.

\begin{tabular}{|l|c|c|}
\hline Distribuição & Notação & Função geradora de densidade $g(u), u \geq 0$ \\
\hline Normal & $N_{m}(\boldsymbol{\mu}, \boldsymbol{\Sigma})$ & $c_{1} \exp (-u / 2)$ \\
t-Student & $t_{m}(\boldsymbol{\mu}, \boldsymbol{\Sigma}, \nu)$ & $c_{2}(1+u / \nu)^{-(\nu+m) / 2}$ \\
$\begin{array}{l}\text { Exponencial } \\
\text { potência }\end{array}$ & $E P_{m}(\boldsymbol{\mu}, \boldsymbol{\Sigma}, \lambda)$ & $c_{3} \exp \left(-u^{\lambda} / 2\right)$ \\
$\begin{array}{l}\text { Normal } \\
\text { contaminada }\end{array}$ & $N C_{m}(\boldsymbol{\mu}, \boldsymbol{\Sigma}, \delta, \tau)$ & $c_{1}\left\{(1-\delta) \exp (-u / 2)+\delta \tau^{-m / 2} \exp (-u / 2 \tau)\right\}$ \\
Logística & $L_{m}(\boldsymbol{\mu}, \boldsymbol{\Sigma})$ & $c_{4} \exp (-u) /(1+\exp (-u))^{2}$ \\
Cauchy & $C_{m}(\boldsymbol{\mu}, \boldsymbol{\Sigma})$ & $c_{5}(1+u)^{(m+1) / 2}$ \\
\hline
\end{tabular}

As expressões $c_{1}, c_{2}, c_{3}, c_{4}$ e $c_{5}$ da Tabela 3.1 não dependem dos parâmetros de interesse, mas podem depender de outros parâmetros que para este trabalho serão considerados conhecidos. Assim, por exemplo, $c_{1}, c_{2}$ e $c_{3}$ são constantes dadas por

$$
c_{1}=-\frac{1}{2}, \quad c_{2}=\nu^{m / 2} k(m, \nu)
$$

$\mathrm{e}$

$$
c_{3}=2^{-\frac{m+2}{\lambda}} m k\left(m-\frac{m+2}{\lambda}, \frac{m+2}{\lambda}\right),
$$

em que $k(\cdot)$ é uma função que depende da dimensão do vetor aleatório $\mathbf{Y}$, com $\nu>2$ e $\lambda \in$ $(0,1]$. Em particular, para o caso da distribuição t-Student essa quantidade dependerá dos graus de liberdades $\nu$, tendo a seguinte forma:

$$
k(m, \nu)=\frac{\Gamma((\nu+m) / 2)}{\pi^{m / 2} \Gamma(\nu / 2)},
$$

em que $\Gamma(t)=\int_{0}^{\infty} x^{t-1} e^{-x} d x$.

No caso da distribuição exponencial potência, a função $k(\cdot)$ depende da dimensão do vetor aleatório e de um parâmetro $\lambda$, associado à curtose da distribuição. Para efeito deste trabalho assumiremos que os parâmetros $\nu$ e $\lambda$ são conhecidos. Assim, também os parâmetros $\delta$ e $\tau$ da distribuição normal contaminada. No Apêndice $\mathrm{C}$ apresentamos a definição da distribuição elíptica bem como algumas propriedades que utilizaremos para a aplicação dos métodos de estimação.

\subsection{Estimação de máxima verossimilhança}

Para o caso do modelo linear elíptico, considerando os resultados obtidos por Osorio et al. (2007), temos que as funções escore são expressas conforme abaixo.

A função escore para $\boldsymbol{\beta}$ é dada por

$$
\frac{\partial}{\partial \boldsymbol{\beta}} l(\boldsymbol{\beta}, \boldsymbol{\alpha} ; \mathbf{Y})=-2 w_{g}(u) \mathbf{X}^{\top} \boldsymbol{\Sigma}^{-1}(\mathbf{Y}-\mathbf{X} \boldsymbol{\beta}),
$$


em que

$$
w_{g}(u)=\frac{d \log g(u)}{d u}=\frac{g^{\prime}(u)}{g(u)}
$$

e $g^{\prime}(u)$ representa a derivada da função $g(\cdot)$, com respeito a $u$.

Enquanto que, a função escore para o $j$-ésimo elemento do vetor $\boldsymbol{\alpha}$ fica dada por

$$
\frac{\partial}{\partial \alpha_{j}} l(\boldsymbol{\beta}, \boldsymbol{\alpha} ; \mathbf{Y})=-\frac{1}{2} \operatorname{tr}\left\{\boldsymbol{\Sigma}^{-1} \frac{\partial \boldsymbol{\Sigma}}{\partial \alpha_{j}}\right\}-w_{g}(u)(\mathbf{Y}-\mathbf{X} \boldsymbol{\beta})^{\top} \boldsymbol{\Sigma}^{-1} \frac{\partial \boldsymbol{\Sigma}}{\partial \alpha_{j}} \boldsymbol{\Sigma}^{-1}(\mathbf{Y}-\mathbf{X} \boldsymbol{\beta})
$$

para $j=1, \ldots, q$. Da equação de estimação para $\boldsymbol{\beta}$ obtemos seu estimador de máxima verossimilhança para $\boldsymbol{\Sigma}$ conhecida, dado por

$$
\hat{\boldsymbol{\beta}}_{M V}=\left(\mathbf{X}^{\top} \boldsymbol{\Sigma}^{-1} \mathbf{X}\right)^{-1} \mathbf{X}^{\top} \boldsymbol{\Sigma}^{-1} \mathbf{Y}
$$

Como $\hat{\boldsymbol{\beta}}_{M V}$ não depende da função geradora $g(\cdot)$, temos assim a mesma expressão para qualquer distribuição elíptica. Mas, se considerarmos o vetor $\boldsymbol{\alpha}$ desconhecido, devemos supor uma estrutura para a matriz $\boldsymbol{\Sigma}$ e na maioria dos casos não teremos um estimador de máxima verossimilhança explícito para a matriz $\boldsymbol{\Sigma}$ e portanto para $\boldsymbol{\beta}$. Assim, da mesma forma que no modelo linear normal precisaremos de algum método de aproximação para encontrarmos uma estimativa de máxima verossimilhança para $\boldsymbol{\Sigma}$ e poder atualizar uma estimativa de máxima verossimilhança para $\boldsymbol{\beta}$, tornando-se um processo iterativo, em que no passo $(r+1)$ teremos

$$
\hat{\boldsymbol{\beta}}_{M V}^{(r+1)}=\left(\mathbf{X}^{\top} \boldsymbol{\Sigma}_{M V}^{-1}{ }^{(r)} \mathbf{X}\right)^{-1} \mathbf{X}^{\top} \boldsymbol{\Sigma}_{M V}^{-1}{ }^{(r)} \mathbf{Y}
$$

para $r=0,1,2, \ldots$. Da mesma forma que foi apresentado no modelo linear normal no Capítulo 2 vamos obter a matriz de informação de Fisher considerando os resultados obtidos por Lange et al. (1989), Savalli et al. (2006) e Osorio et al. (2007). Temos que a matriz de informação de Fisher é uma matriz diagonal por bloco dada por

$$
\mathbf{K}(\boldsymbol{\beta}, \boldsymbol{\alpha})=\left[\begin{array}{cc}
\mathbf{K}_{\beta \beta} & \mathbf{0} \\
\mathbf{0} & \mathbf{K}_{\alpha \alpha}
\end{array}\right]
$$

em que

$$
\mathbf{K}_{\beta \beta}=\left(\frac{4 d_{g}}{m}\right) \mathbf{X}^{\top} \boldsymbol{\Sigma}^{-1} \mathbf{X}
$$

$d_{g}=\mathrm{E}\left\{w_{g}^{2}(u) u\right\}$ e o $(j, k)$-ésimo elemento da matriz $\mathbf{K}_{\alpha \alpha}$ fica dado por

$$
\mathbf{K}_{\alpha_{j} \alpha_{k}}=\frac{b_{j, k}}{4}\left(\frac{4 f_{g}}{m(m+2)}-1\right)+\frac{2 f_{g}}{m(m+2)} \operatorname{tr}\left\{\boldsymbol{\Sigma}^{-1} \frac{\partial \boldsymbol{\Sigma}}{\partial \alpha_{j}} \boldsymbol{\Sigma}^{-1} \frac{\partial \boldsymbol{\Sigma}}{\partial \alpha_{k}}\right\}
$$

em que $f_{g}=\mathrm{E}\left\{w_{g}^{2}(u) u^{2}\right\}$ e $b_{j, k}$ é $\operatorname{tr}\left\{\boldsymbol{\Sigma}^{-1} \frac{\partial \boldsymbol{\Sigma}}{\partial \alpha_{j}}\right\} \operatorname{tr}\left\{\boldsymbol{\Sigma}^{-1} \frac{\partial \boldsymbol{\Sigma}}{\partial \alpha_{k}}\right\}, \operatorname{com} j, k$ em $1, \ldots, q$.

\subsection{Estimação de máxima verossimilhança restrita}

Nesta seção mostramos que a transformação proposta por Verbyla (1990) que foi apresentada na Seção 2.3 pode ser implementada na estimação de um modelo linear elíptico. Uma forma de obter as matrizes de variancia e covariancia assintóticas para os estimadores de máxima verossimilhança é considerando as inversas das matrizes de informação de Fisher. 


\subsubsection{Método proposto por Verbyla}

Similarmente ao caso normal, uma transformação linear de uma variável com distribuição elíptica continua sendo elíptica e tanto a distribuição condicional como a distribuição marginal das variáveis com distribuição elíptica, também, continuam pertencendo à família elíptica (ver Apêndice C). Então, podemos considerar a decomposição do logaritmo da função de verossimilhança da transformação proposta por Verbyla (1990) e o método de estimação proposto por Patterson e Thompson (1971).

Lembremos que $\mathbf{Y} \sim E l_{m}(\boldsymbol{\mu}, \boldsymbol{\Sigma}, g) \operatorname{com} \boldsymbol{\mu}=\mathbf{X} \boldsymbol{\beta}$ e $\boldsymbol{\Sigma}=\boldsymbol{\Sigma}(\boldsymbol{\alpha})$, em que o vector de parâmetros $\boldsymbol{\alpha}$ define a estrutura de $\boldsymbol{\Sigma}$ com elementos $\left(\alpha_{1}, \ldots, \alpha_{j}, \ldots, \alpha_{q}\right)$ com $q<m-p$. Então, consideramos a transformação $\mathbf{W}^{\top} \mathbf{Y}$, com $\mathbf{W}=\left[\mathbf{W}_{1}, \mathbf{W}_{2}\right]$ de dimensão $m \times m$, em que $\mathbf{W}_{1}$ e $\mathbf{W}_{2}$ devem seguir as condições

$$
\mathbf{W}_{1}^{\top} \mathbf{X}=\mathbf{I}_{p} \quad e \quad \mathbf{W}_{2}^{\top} \mathbf{X}=\mathbf{0} .
$$

Como precisamos apenas especificar a estrutura da matriz $\mathbf{W}_{1}$, novamente utilizaremos a estrutura proposta por Verbyla (1990), isto é, $\mathbf{W}_{1}=\left(\mathbf{X}^{\top} \mathbf{X}\right)^{-1} \mathbf{X}^{\top}$. Sendo $\mathbf{Y}$ um vetor aleatório com distribuição elíptica, a transformação terá a seguinte distribuição:

$$
\mathbf{W}^{\top} \mathbf{Y} \sim E l_{m}\left(\mathbf{W}^{\top} \boldsymbol{\mu}, \mathbf{W}^{\top} \boldsymbol{\Sigma} \mathbf{W}, g\right) .
$$

Dado que a transformação não muda a dimensão da distribuição e se a matriz $\mathbf{W}^{\top} \boldsymbol{\Sigma} \mathbf{W}$ é positiva definida a função de densidade da transformação será determinada pela função característica da densidade $g(\cdot)$. De outro modo, se a matriz $\mathbf{W}^{\top} \boldsymbol{\Sigma} \mathbf{W}$ não é positiva definida não terá função de densidade, mas a distribuição de $\mathbf{W}^{\top} \mathbf{Y}$ será determinada por uma função $\phi$ que define a função característica de uma distribuição elíptica (ver Definição C.1.1 do Apêndice C). Isto é, a transformação continua pertencendo à família elíptica. Também podemos particionar da seguinte forma:

$$
\left[\begin{array}{c}
\mathbf{V}_{1} \\
\mathbf{V}_{2}
\end{array}\right] \sim E l_{m}\left\{\left[\begin{array}{c}
\boldsymbol{\beta} \\
\mathbf{0}
\end{array}\right],\left[\begin{array}{ll}
\mathbf{W}_{1}^{\top} \mathbf{\Sigma} \mathbf{W}_{1} & \mathbf{W}_{1}^{\top} \mathbf{\Sigma} \mathbf{W}_{2} \\
\mathbf{W}_{2}^{\top} \boldsymbol{\Sigma} \mathbf{W}_{1} & \mathbf{W}_{2}^{\top} \boldsymbol{\Sigma} \mathbf{W}_{2}
\end{array}\right], g\right\}
$$

Assim, como as variáveis $\mathbf{V}_{1}$ e $\mathbf{V}_{2}$ provêm da transformação da variável $\mathbf{Y}$, podemos determinar as funções de densidade marginal de $\mathbf{V}_{2}$ e condicional de $\mathbf{V}_{1} \mid \mathbf{V}_{2}$, se essas existirem. Denotaremos

$$
\begin{gathered}
\mathbf{W}_{2}^{\top} \mathbf{Y}=\mathbf{V}_{2} \sim E l_{m-p}\left(\mathbf{0}, \mathbf{W}_{2}^{\top} \boldsymbol{\Sigma} \mathbf{W}_{2}, g_{2}\right), \\
\mathbf{V}_{1} \mid \mathbf{V}_{2} \sim E l_{p}\left(\boldsymbol{\beta}+\mathbf{W}_{1}^{\top} \boldsymbol{\Sigma} \mathbf{W}_{2}\left(\mathbf{W}_{2}^{\top} \boldsymbol{\Sigma} \mathbf{W}_{2}\right)^{-1} \mathbf{W}_{2} \mathbf{Y}, \mathbf{W}_{1}^{\top} \mathbf{X}\left(\mathbf{X}^{\top} \boldsymbol{\Sigma}^{-1} \mathbf{X}\right)^{-1} \mathbf{X}^{\top} \mathbf{W}_{1}, g_{v_{1}}\right)
\end{gathered}
$$

em que $g_{2}(\cdot)$ e $g_{v_{1}}(\cdot)$ são as funções geradoras de densidade das distribuições elípticas de $\mathbf{V}_{2}$ e de $\mathbf{V}_{1}$ dado $\mathbf{V}_{2}$, respectivamente.

\section{Determinação das funções geradoras de densidade}

Para determinar a função $g_{2}(\cdot)$ vamos considerar as propriedades das distribuições elípticas dadas na Tese de Doutorado de Arellano (1994) e brevemente explicadas no Âpendice C. Consideremos que por definição de uma distribuição elíptica a função de densidade de $\mathbf{V}_{2}$ pode ser escrita como

$$
f_{2}\left(\mathbf{v}_{2}\right)=\left|\mathbf{W}_{2}^{\top} \boldsymbol{\Sigma} \mathbf{W}_{2}\right|^{-1 / 2} g_{2}\left(u_{2}\right),
$$

em que $u_{2}$ denota a forma quadrática associada à distribuição marginal de $\mathbf{V}_{2}$. Esta densidade também pode ser obtida integrando a função de densidade conjunta de $\mathbf{W}^{\top} \mathbf{Y}$ com respeito a $\mathbf{V}_{1}$,

$$
f_{2}\left(\mathbf{v}_{2}\right)=\int_{R^{m-p}} f(\mathbf{v}) d \mathbf{V}_{1}=\int_{R^{m-p}}\left|\mathbf{W}^{\top} \mathbf{\Sigma} \mathbf{W}\right|^{-1 / 2} g\left(u_{v}\right) d \mathbf{V}_{1},
$$


aqui, $f(\mathbf{v})$ denota a função de densidade conjunta da transformação $\mathbf{W}^{\top} \mathbf{Y}$ e $u_{v}$ denota sua respectiva forma quadrática. Assim, a função geradora de densidade de $\mathbf{V}_{2}$ fica dada por

$$
g_{2}\left(u_{2}\right)=\left|\mathbf{W}_{2}^{\top} \boldsymbol{\Sigma} \mathbf{W}_{2}\right|^{1 / 2} \int_{R^{m-p}}\left|\mathbf{W}^{\top} \boldsymbol{\Sigma} \mathbf{W}\right|^{-1 / 2} g\left(u_{v}\right) d \mathbf{V}_{1} .
$$

Enquanto que, para obter a função geradora da distribuição condicional $\mathbf{V}_{1} \mid \mathbf{V}_{2}$, temos por definição de uma distribuição elíptica que sua função de densidade é dada por

$$
f_{v_{1}}\left(\mathbf{v}_{1}\right)=\left|\mathbf{W}_{1}^{\top} \mathbf{X}\left(\mathbf{X}^{\top} \boldsymbol{\Sigma}^{-1} \mathbf{X}\right)^{-1} \mathbf{X}^{\top} \mathbf{W}_{1}\right|^{-1 / 2} g_{v 1}\left(u_{c}\right),
$$

em que $u_{c}$ é a forma quadrática associada à variável condicional $\mathbf{V}_{1} \mid \mathbf{V}_{2}$. Por definição, uma função de densidade condicional pode ser escrita como o quociente entre a função de densidade conjunta e a função de densidade marginal da variável condicionada. Neste caso temos

$$
f_{v 1}\left(\mathbf{v}_{1}\right)=\frac{\left|\mathbf{W}^{\top} \boldsymbol{\Sigma} \mathbf{W}\right|^{-1 / 2} g\left(u_{v}\right)}{\left|\mathbf{W}_{2}^{\top} \boldsymbol{\Sigma} \mathbf{W}_{2}\right|^{-1 / 2} g_{2}\left(u_{2}\right)}=\left|\mathbf{X}^{\top} \boldsymbol{\Sigma}^{-1} \mathbf{X}\right|^{-1 / 2} \frac{g\left(u_{v}\right)}{g_{2}\left(u_{2}\right)} .
$$

Nessa última igualdade consideramos a decomposição do determinante descrita na equação (A.6). Portanto, a função geradora de densidade da distribuição condicional $\mathbf{V}_{1} \mid \mathbf{V}_{2}$ fica dada por

$$
g_{v 1}\left(u_{c}\right)=\left|\mathbf{W}_{1}^{\top} \mathbf{X}\left(\mathbf{X}^{\top} \boldsymbol{\Sigma}^{-1} \mathbf{X}\right)^{-1} \mathbf{X}^{\top} \mathbf{W}_{1}\right|^{1 / 2}\left|\mathbf{X}^{\top} \boldsymbol{\Sigma}^{-1} \mathbf{X}\right|^{-1 / 2} \frac{g\left(u_{v}\right)}{g_{2}\left(u_{2}\right)} .
$$

Para calcular as formas quadráticas, temos que $u_{c}$ é a forma quadrática associada à distribuição condicional de $\mathbf{V}_{1}$ dado $\mathbf{V}_{2}$, sendo dada por

$$
u_{c}=\left\{\mathbf{V}_{1}-\boldsymbol{\mu}_{1}\left(\mathbf{v}_{2}\right)\right\}^{\top}\left\{\mathbf{W}_{1}^{\top} \mathbf{X}\left(\mathbf{X}^{\top} \boldsymbol{\Sigma}^{-1} \mathbf{X}\right)^{-1} \mathbf{X}^{\top} \mathbf{W}_{1}\right\}^{-1}\left\{\mathbf{V}_{1}-\boldsymbol{\mu}_{1}\left(\mathbf{v}_{2}\right)\right\},
$$

em que $\mathbf{v}_{2}$ representa um valor da variável $\mathbf{V}_{2}$. Enquanto que,

$$
u_{2}=\mathbf{Y}^{\top} \mathbf{W}_{2}\left(\mathbf{W}_{2}^{\top} \boldsymbol{\Sigma} \mathbf{W}_{2}\right)^{-1} \mathbf{W}_{2}^{\top} \mathbf{Y} .
$$

Assim, podemos escrever $u_{v}$, a forma quadrática da transformação $\mathbf{W}^{\top} \mathbf{Y}$, como a decomposição de

$$
\begin{aligned}
u_{c}+u_{2}= & \left\{\mathbf{V}_{1}-\boldsymbol{\mu}_{1}\left(\mathbf{v}_{2}\right)\right\}^{\top}\left\{\mathbf{W}_{1}^{\top} \mathbf{X}\left(\mathbf{X}^{\top} \boldsymbol{\Sigma}^{-1} \mathbf{X}\right)^{-1} \mathbf{X}^{\top} \mathbf{W}_{1}\right\}^{-1}\left\{\mathbf{V}_{1}-\boldsymbol{\mu}_{1}\left(\mathbf{v}_{2}\right)\right\} \\
& +\mathbf{Y}^{\top} \mathbf{W}_{2}\left(\mathbf{W}_{2}^{\top} \boldsymbol{\Sigma} \mathbf{W}_{2}\right)^{-1} \mathbf{W}_{2}^{\top} \mathbf{Y} .
\end{aligned}
$$

Por último, considerando para $u_{c}$ o resultado da equação (B.4), a forma de escrever $u_{2}$ usando o resultado da equação (A.4) e $\mathbf{b}=\left(\mathbf{X}^{\top} \boldsymbol{\Sigma}^{-1} \mathbf{X}\right)^{-1} \mathbf{X}^{\top} \boldsymbol{\Sigma}^{-1} \mathbf{Y}$, temos que

$$
u_{v}=u_{c}+u_{2}=(\mathbf{Y}-\mathbf{X} \boldsymbol{\beta})^{\top} \boldsymbol{\Sigma}^{-1}(\mathbf{Y}-\mathbf{X} \boldsymbol{\beta})=u .
$$

Portanto, a forma quadrática $u_{v}$, associada à transformação de $\mathbf{W}^{\top} \mathbf{Y}$, nada mais é do que a forma quadrática, $u$, associada à distribuição $\mathbf{Y}$. Em outras palavras, a transformação $\mathbf{W}^{\top} \mathbf{Y}$ não afeta a informação dos dados originais permitindo particionar a função de verossimilhança, obtendo uma verossimilhança para os componentes de variância.

\section{Função de verosimilhança restrita}

Podemos expressar o logaritmo da função de verossimilhança para os parâmetros $\boldsymbol{\beta}$ e $\boldsymbol{\alpha}$ da transformação $\mathbf{W}^{\top} \mathbf{Y}$ como

$$
l\left(\boldsymbol{\beta}, \boldsymbol{\alpha} ; \mathbf{V}_{1}, \mathbf{V}_{2}\right)=l\left(\boldsymbol{\beta}, \boldsymbol{\alpha} ; \mathbf{V}_{1} \mid \mathbf{V}_{2}\right)+l\left(\boldsymbol{\alpha} ; \mathbf{V}_{2}\right),
$$


em que o logaritmo da função de verossimilhança da condicional $\mathbf{V}_{1} \mid \mathbf{V}_{2}$ fica dado por

$$
l\left(\boldsymbol{\beta}, \boldsymbol{\alpha} ; \mathbf{V}_{1} \mid \mathbf{V}_{2}\right)=-\frac{1}{2} \log \left|\mathbf{W}_{1}^{\top} \mathbf{X}\left(\mathbf{X}^{\top} \boldsymbol{\Sigma}^{-1} \mathbf{X}\right)^{-1} \mathbf{X}^{\top} \mathbf{W}_{1}\right|+\log g_{v_{1}}\left(u_{c}\right),
$$

enquanto que o logaritmo da função de verossimilhança da marginal de $\mathbf{V}_{2}$ fica dado por

$$
l\left(\boldsymbol{\alpha} ; \mathbf{V}_{2}\right)=-\frac{1}{2} \log \left|\mathbf{W}_{2}^{\top} \boldsymbol{\Sigma} \mathbf{W}_{2}\right|+\log g_{2}\left(u_{2}\right) .
$$

Assim, como foi dito na Seção 2.3 do capítulo anterior, ao maximizarmos $l\left(\boldsymbol{\beta}, \boldsymbol{\alpha} ; \mathbf{V}_{1} \mid \mathbf{V}_{2}\right)$ com respeito a $\boldsymbol{\beta}$ vamos obter o estimador de $\boldsymbol{\beta}$. Então, considerando os resultados das equações (3.10) e (3.12), a derivada do logaritmo da função de verossimilhança condicional de $\mathbf{V}_{1} \mid \mathbf{V}_{2}$ com respeito de $\boldsymbol{\beta}$ fica dada por

$$
\frac{\partial l\left(\boldsymbol{\beta}, \boldsymbol{\alpha} ; \mathbf{V}_{1} \mid \mathbf{V}_{2}\right)}{\partial \boldsymbol{\beta}}=-2 w_{g}(u)\left(\mathbf{X}^{\top} \boldsymbol{\Sigma}^{-1} \mathbf{X}\right)(\mathbf{b}-\boldsymbol{\beta})
$$

em que $\mathbf{b}=\left(\mathbf{X}^{\top} \boldsymbol{\Sigma}^{-1} \mathbf{X}\right)^{-1} \mathbf{X}^{\top} \boldsymbol{\Sigma}^{-1} \mathbf{Y}$, e $w_{g}(u)$ como expresso em (3.6). Igualando a zero a equação (3.14) para um valor de $\boldsymbol{\Sigma}$ fixo, obtemos o seguinte estimador para $\boldsymbol{\beta}$ para este método:

$$
\hat{\boldsymbol{\beta}}=\left(\mathbf{X}^{\top} \boldsymbol{\Sigma}^{-1} \mathbf{X}\right)^{-1} \mathbf{X}^{\top} \boldsymbol{\Sigma}^{-1} \mathbf{Y} .
$$

Vemos que a estrutura deste estimador de $\boldsymbol{\beta}$ é exatamente o estimador de máxima verossimilhança para $\boldsymbol{\beta}$ do modelo elíptico para um $\boldsymbol{\Sigma}$ fixo, obtido na Seção 3.2, isto é, ao consideramos $\boldsymbol{\Sigma}$ conhecido recuperamos o estimador de máxima verossimilhança para $\boldsymbol{\beta}$.

De modo mais geral, dado que mediante a transformação continuamos num modelo elíptico, para $\boldsymbol{\alpha}$ desconhecido, a igualdade das estruturas dos estimadores é um resultado esperado uma vez que o estimador de máxima verossimilhança de $\boldsymbol{\beta}$, num modelo elíptico, não depende da função geradora.

Além disso, devemos considerar que da mesma forma que na distribuição normal, o estimador nem sequer tem sido modificado pela transformação das variáveis. Isto é, a transformação considerada neste método de estimação não afeta a estrutura do estimador de $\boldsymbol{\beta}$. Dado que o estimador de $\boldsymbol{\beta}$ depende do vetor $\boldsymbol{\alpha}$ desconhecido, uma vez que determinemos a estimativa da matriz $\boldsymbol{\Sigma}$ obteremos a estimativa do vetor $\boldsymbol{\beta}$. Seguindo com o método de estimação proposto por Verbyla (1990), devemos maximizar $l\left(\boldsymbol{\alpha} ; \mathbf{V}_{2}\right)$ com respeito a $\alpha_{j}$ e utilizaremos a decomposição do determinante da matriz de escala apresentada na equação (A.8). Assim, obtemos

$$
\begin{aligned}
\frac{\partial l\left(\boldsymbol{\alpha} ; \mathbf{V}_{2}\right)}{\partial \alpha_{j}}= & \frac{1}{2} \operatorname{tr}\left\{\left(\mathbf{X}^{\top} \boldsymbol{\Sigma}^{-1} \mathbf{X}\right)^{-1} \mathbf{X}^{\top} \boldsymbol{\Sigma}^{-1} \frac{\partial \boldsymbol{\Sigma}}{\partial \alpha_{j}} \boldsymbol{\Sigma}^{-1} \mathbf{X}\right\}-\frac{1}{2} \operatorname{tr}\left\{\boldsymbol{\Sigma}^{-1} \frac{\partial \boldsymbol{\Sigma}}{\partial \alpha_{j}}\right\} \\
& -w_{2}\left(u_{2}\right)(\mathbf{Y}-\mathbf{X} \mathbf{b})^{\top} \boldsymbol{\Sigma}^{-1} \frac{\partial \boldsymbol{\Sigma}}{\partial \alpha_{j}} \boldsymbol{\Sigma}^{-1}(\mathbf{Y}-\mathbf{X b}),
\end{aligned}
$$

para $j=1, \ldots, q$, em que $w_{2}\left(u_{2}\right)=d \log g_{2}\left(u_{2}\right) / d u_{2}=g_{2}^{\prime}\left(u_{2}\right) / g_{2}\left(u_{2}\right)$ e lembrando que $u_{2}=$ $(\mathbf{Y}-\mathbf{X b})^{\top} \boldsymbol{\Sigma}^{-1}(\mathbf{Y}-\mathbf{X b})$.

Vemos que o estimador da matriz de escala não tem forma explícita e deve ser obtido com base em algum método de aproximação. Assim, podemos obter um estimador de máxima verossimilhança restrita para $\boldsymbol{\Sigma}$ da maximização do logaritmo da função de verossimilhança marginal de $\mathbf{V}_{2}$ dada na equação (3.13) e por conseguinte para $\boldsymbol{\beta}$ dada por

$$
\hat{\boldsymbol{\beta}}_{M V R}=\left(\mathbf{X}^{\top}\left(\widehat{\boldsymbol{\Sigma}}_{M V R}\right)^{-1} \mathbf{X}\right)^{-1} \mathbf{X}^{\top}\left(\widehat{\boldsymbol{\Sigma}}_{M V R}\right)^{-1} \mathbf{Y}
$$

em que $\widehat{\boldsymbol{\Sigma}}_{M V R}$ denota a estimativa de máxima verossimilhança restrita de $\boldsymbol{\Sigma}$. Até agora concluímos que, em virtude de que a transformação de WY tem distribuição elíptica e que a estrutura do estimador de $\boldsymbol{\beta}$ é a mesma para qualquer distribuição elíptica (não depende da função $g(\cdot)$ ), o estimador restrito de $\boldsymbol{\beta}$ tem a mesma estrutura que o estimador de máxima verossimilhança. A 
estimativa restrita da matriz $\boldsymbol{\Sigma}$ deve ser obtida maximizando o logaritmo da função de verossimilhança restrita, marginal de $\mathbf{V}_{2}$, com respeito ao vetor $\boldsymbol{\alpha}$ dada na equação (3.16). No entanto, as estimativas de $\boldsymbol{\beta}$ e $\boldsymbol{\Sigma}$ pelo método de máxima verossimilhança devem ser obtidas num processo iterativo.

\subsection{Estimação baseada na verossimilhança perfilada modificada}

Na Seção 2.4 definimos a função de verossimilhança modificada ajustada e vimos os resultados para o modelo linear normal. Para o modelo linear elíptico o estimador de máxima verossimilhança de $\boldsymbol{\beta}$ tem a mesma estrutura para todas as distribuições elípticas. Então, considerando o vetor $\boldsymbol{\beta}$ como de parâmetros de incômodo, temos que o estimador de máxima verossimilhança parcial de $\boldsymbol{\beta}$ é dado por $\hat{\boldsymbol{\beta}}_{\alpha}=\left(\mathbf{X}^{\top} \boldsymbol{\Sigma}^{-1} \mathbf{X}\right)^{-1} \mathbf{X}^{\top} \boldsymbol{\Sigma}^{-1} \mathbf{Y}$ e o logaritmo da função de verossimilhança perfilada de $\boldsymbol{\alpha}$, é dado por

$$
l_{p}(\boldsymbol{\alpha})=-\frac{1}{2} \log |\boldsymbol{\Sigma}|+\log g\left\{\left(\mathbf{Y}-\mathbf{X} \hat{\boldsymbol{\beta}}_{\alpha}\right)^{\top} \boldsymbol{\Sigma}^{-1}\left(\mathbf{Y}-\mathbf{X} \hat{\boldsymbol{\beta}}_{\alpha}\right)\right\} .
$$

A matriz de informação observada com respeito a $\boldsymbol{\beta}$ é obtida considerando o resultado de (3.5) em que obtivemos a primeira derivada com respeito a $\boldsymbol{\beta}$, e a segunda derivada fica dada por

$$
\begin{aligned}
\frac{\partial^{2}}{\partial \boldsymbol{\beta} \partial \boldsymbol{\beta}^{\top}} l(\boldsymbol{\beta}, \boldsymbol{\alpha} ; \mathbf{Y}) & =\frac{\partial}{\partial \boldsymbol{\beta}^{\top}}\left(-2 w_{g}(u) \mathbf{X}^{\top} \boldsymbol{\Sigma}^{-1}(\mathbf{Y}-\mathbf{X} \boldsymbol{\beta})\right) \\
& =4 w_{g}^{\prime}(u) \mathbf{X}^{\top} \boldsymbol{\Sigma}^{-1}(\mathbf{Y}-\mathbf{X} \boldsymbol{\beta})(\mathbf{Y}-\mathbf{X} \boldsymbol{\beta})^{\top} \boldsymbol{\Sigma}^{-1} \mathbf{X}+2 w_{g}(u) \mathbf{X}^{\top} \boldsymbol{\Sigma}^{-1} \mathbf{X},
\end{aligned}
$$

em que $w_{g}^{\prime}(u)$ denota a segunda derivada da função $\log \{g(u)\}$.

\subsubsection{Ajuste da função de verossimilhança perfilada modificada}

A matriz de informação observada com respeito a $\boldsymbol{\beta}$ avaliada no estimador de máxima verossimilhança parcial de $\boldsymbol{\beta}$ fica dada por

$$
\begin{aligned}
\mathbf{J}_{\beta \beta}\left(\hat{\boldsymbol{\beta}}_{\alpha}, \boldsymbol{\alpha}\right) & =4 w_{g}^{\prime}\left(\hat{u}_{\alpha}\right) \mathbf{X}^{\top} \boldsymbol{\Sigma}^{-1}\left(\mathbf{Y}-\mathbf{X} \hat{\boldsymbol{\beta}}_{\alpha}\right)\left(\mathbf{Y}-\mathbf{X} \hat{\boldsymbol{\beta}}_{\alpha}\right)^{\top} \boldsymbol{\Sigma}^{-1} \mathbf{X}+2 w_{g}\left(\hat{u}_{\alpha}\right) \mathbf{X}^{\top} \boldsymbol{\Sigma}^{-1} \mathbf{X} \\
& =4 w_{g}^{\prime}\left(\hat{u}_{\alpha}\right)\left(\mathbf{X}^{\top} \boldsymbol{\Sigma}^{-1} \mathbf{Y}-\mathbf{X}^{\top} \boldsymbol{\Sigma}^{-1} \mathbf{X} \hat{\boldsymbol{\beta}}_{\alpha}\right)\left(\mathbf{Y}^{\top} \boldsymbol{\Sigma}^{-1} \mathbf{X}-\hat{\boldsymbol{\beta}}_{\alpha}^{\top} \mathbf{X}^{\top} \boldsymbol{\Sigma}^{-1} \mathbf{X}\right)+2 w_{g}\left(\hat{u}_{\alpha}\right) \mathbf{X}^{\top} \boldsymbol{\Sigma}^{-1} \mathbf{X} \\
& =2 w_{g}\left(\hat{u}_{\alpha}\right) \mathbf{X}^{\top} \boldsymbol{\Sigma}^{-1} \mathbf{X},
\end{aligned}
$$

em que a expressão $\hat{u}_{\alpha}$ é dada por $\left(\mathbf{Y}-\mathbf{X} \hat{\boldsymbol{\beta}}_{\alpha}\right)^{\top} \boldsymbol{\Sigma}^{-1}\left(\mathbf{Y}-\mathbf{X} \hat{\boldsymbol{\beta}}_{\alpha}\right)$. Na última igualdade utilizamos o resultado da equação (B.5), em que $\left(\mathbf{X}^{\top} \boldsymbol{\Sigma}^{-1} \mathbf{Y}-\mathbf{X}^{\top} \boldsymbol{\Sigma}^{-1} \mathbf{X} \hat{\boldsymbol{\beta}}_{\alpha}\right)=\mathbf{0}$. Portanto, similar a (2.10), o ajuste da função de verossimilhança perfilada modificada de $\boldsymbol{\beta}$ fica dado por

$$
\begin{aligned}
l_{p m}^{*}(\boldsymbol{\alpha}) & =l_{p}(\boldsymbol{\alpha})-\frac{1}{2} \log \left\{\left|\mathbf{J}_{\beta \beta}\left(\hat{\boldsymbol{\beta}}_{\alpha}, \boldsymbol{\alpha}\right)\right|\right\} \\
& \left.=l_{p}(\boldsymbol{\alpha})-\frac{1}{2} \log \left\{\mid 2 w_{g}\left(\hat{u}_{\alpha}\right) \mathbf{X}^{\top} \boldsymbol{\Sigma}^{-1} \mathbf{X}\right\} \mid\right\}
\end{aligned}
$$

com $l_{p}(\boldsymbol{\alpha})$ dado em (3.17). Assim, derivando com respeito ao $j$-ésimo elemento de $\boldsymbol{\alpha}$ obtemos

$$
\left.\frac{\partial}{\partial \alpha_{j}} l_{p m}^{*}(\boldsymbol{\alpha})=-\frac{1}{2} \operatorname{tr}\left\{\boldsymbol{\Sigma}^{-1} \frac{\partial \boldsymbol{\Sigma}}{\partial \alpha_{j}}\right\}+w_{g}\left(\hat{u}_{\alpha}\right)\left[\frac{\partial \hat{u}_{\alpha}}{\partial \alpha_{j}}\right]-\frac{1}{2} \frac{\partial}{\partial \alpha_{j}}\left[\log \left\{\mid 2 w_{g}\left(\hat{u}_{\alpha}\right) \mathbf{X}^{\top} \boldsymbol{\Sigma}^{-1} \mathbf{X}\right\} \mid\right\}\right],
$$


em que a derivada parcial do último termo fica dada por

$$
\begin{aligned}
\left.-\frac{1}{2} \frac{\partial}{\partial \alpha_{j}}\left[\log \left\{\mid 2 w_{g}\left(\hat{u}_{\alpha}\right) \mathbf{X}^{\top} \boldsymbol{\Sigma}^{-1} \mathbf{X}\right\} \mid\right\}\right] & =-\frac{p}{2} \frac{\partial}{\partial \alpha_{j}}\left[\log \left\{2 w_{g}\left(\hat{u}_{\alpha}\right)\right\}\right]+\frac{1}{2} \frac{\partial}{\partial \alpha_{j}}\left[\log \left\{\left|\mathbf{X}^{\top} \boldsymbol{\Sigma}^{-1} \mathbf{X}\right|\right\}\right] \\
& =-\frac{p}{2} \frac{w_{g}^{\prime}\left(\hat{u}_{\alpha}\right)}{w_{g}\left(\hat{u}_{\alpha}\right)}\left[\frac{\partial \hat{u}_{\alpha}}{\partial \alpha_{j}}\right]+\frac{1}{2} \operatorname{tr}\left\{\left(\mathbf{X}^{\top} \boldsymbol{\Sigma}^{-1} \mathbf{X}\right)^{-1} \mathbf{X}^{\top} \boldsymbol{\Sigma}^{-1} \frac{\partial \boldsymbol{\Sigma}}{\partial \alpha_{j}} \boldsymbol{\Sigma}^{-1} \mathbf{X}\right\}
\end{aligned}
$$

para $j=1, \ldots, q$ e a derivada parcial de $\hat{u}_{\alpha}$ com respeito a $\alpha_{j}$ é dada por $-\left(\mathbf{Y}-\mathbf{X} \hat{\boldsymbol{\beta}}_{\alpha}\right)^{\top} \boldsymbol{\Sigma}^{-1} \frac{\partial \boldsymbol{\Sigma}}{\partial \alpha_{j}} \boldsymbol{\Sigma}^{-1}(\mathbf{Y}-$ $\mathbf{X} \hat{\boldsymbol{\beta}}_{\alpha}$ ) (ver a equação (B.6) no Apêndice B). Então, a derivada parcial da função de máxima verossimilhança perfilada modificada de $\boldsymbol{\alpha}$ com respeito ao $j$-ésimo elemento de $\boldsymbol{\alpha}$ fica dada por

$$
\begin{aligned}
\frac{\partial}{\partial \alpha_{j}} l_{p m}^{*}(\boldsymbol{\alpha}) & =-\frac{1}{2} \operatorname{tr}\left\{\boldsymbol{\Sigma}^{-1} \frac{\partial \boldsymbol{\Sigma}}{\partial \alpha_{j}}\right\}+\frac{1}{2} \operatorname{tr}\left\{\left(\mathbf{X}^{\top} \boldsymbol{\Sigma}^{-1} \mathbf{X}\right)^{-1} \mathbf{X}^{\top} \boldsymbol{\Sigma}^{-1} \frac{\partial \boldsymbol{\Sigma}}{\partial \alpha_{j}} \boldsymbol{\Sigma}^{-1} \mathbf{X}\right\} \\
& +\frac{2 w_{g}\left(\hat{u}_{\alpha}\right)^{2}-p w_{g}^{\prime}\left(\hat{u}_{\alpha}\right)}{2 w_{g}\left(\hat{u}_{\alpha}\right)}\left[\frac{\partial \hat{u}_{\alpha}}{\partial \alpha_{j}}\right] .
\end{aligned}
$$

Por último, escrevemos $C_{g}(\boldsymbol{\alpha})$ como o termo $\left\{2 w_{g}\left(\hat{u}_{\alpha}\right)^{2}-p w_{g}^{\prime}\left(\hat{u}_{\alpha}\right)\right\} / 2 w_{g}\left(\hat{u}_{\alpha}\right)$ e assim a equação de estimação para $\alpha_{j}$ fica dada por

$$
\frac{\partial}{\partial \alpha_{j}} l_{p m}^{*}(\boldsymbol{\alpha})=-\frac{1}{2} \operatorname{tr}\left\{\boldsymbol{\Sigma}^{-1} \frac{\partial \boldsymbol{\Sigma}}{\partial \alpha_{j}}\right\}+\frac{1}{2} \operatorname{tr}\left\{\left(\mathbf{X}^{\top} \boldsymbol{\Sigma}^{-1} \mathbf{X}\right)^{-1} \mathbf{X}^{\top} \boldsymbol{\Sigma}^{-1} \frac{\partial \boldsymbol{\Sigma}}{\partial \alpha_{j}} \boldsymbol{\Sigma}^{-1} \mathbf{X}\right\}+C_{g}(\boldsymbol{\alpha})\left[\frac{\partial \hat{u}_{\alpha}}{\partial \alpha_{j}}\right],
$$

para $j=1, \ldots, q$. Desta forma, vemos que o único termo que dependerá da função geradora de densidade $g(\cdot)$ é $C_{g}(\boldsymbol{\alpha})$, portanto é o único termo que vai mudar dependendo da distribuição elíptica que seja considerada.

Outra aproximação para a função de verossimilhança perfilada modificada pode ser considerada substituindo a matriz de informação observada pela matriz de informação esperada de $\boldsymbol{\beta}$. Na próxima seção apresentamos essa aproximação utilizando os resultados apresentados por Lange et al. (1989) com respeito à matriz de informação esperada de uma distribuição elíptica.

\subsubsection{Ajuste da função verossimilhança perfilada modificada aproximada}

Consideremos dois elementos do vetor de parâmetros $\boldsymbol{\beta}$, sejam $\beta_{k}$ e $\beta_{l}$ com $k \neq l$. Além disso, dado que $\boldsymbol{\Sigma}$ é uma matriz simétrica e pode ser decomposta como $\boldsymbol{\Sigma}^{-1}=\boldsymbol{\Sigma}^{-1 / 2} \boldsymbol{\Sigma}^{-1 / 2}$ (ver detalhes na Seção A.1 do Apêndice A), definimos a variável $\mathbf{z}=\boldsymbol{\Sigma}^{-1 / 2}(\mathbf{Y}-\mathbf{X} \boldsymbol{\beta})$ e $\mathbf{X}_{k}$ como sendo a $k$-ésima coluna da matriz $\mathbf{X}$, com $k=1, \cdots, p$. Então, obtemos

$$
\begin{aligned}
\frac{\partial l(\boldsymbol{\beta}, \boldsymbol{\alpha} ; \mathbf{Y})}{\partial \beta_{k}} \frac{\partial l(\boldsymbol{\beta}, \boldsymbol{\alpha} ; \mathbf{Y})}{\partial \beta_{l}} & =\left\{-2 w_{g}(u) \mathbf{X}_{k}^{\top} \boldsymbol{\Sigma}^{-1}(\mathbf{Y}-\mathbf{X} \boldsymbol{\beta})\right\}\left\{-2 w_{g}(u) \mathbf{X}_{l}^{\top} \boldsymbol{\Sigma}^{-1}(\mathbf{Y}-\mathbf{X} \boldsymbol{\beta})\right\} \\
& =4 w_{g}^{2}(u) \mathbf{z}^{\top} \boldsymbol{\Sigma}^{-1 / 2} \mathbf{X}_{k} \mathbf{X}_{l}^{\top} \boldsymbol{\Sigma}^{-1 / 2} \mathbf{z},
\end{aligned}
$$

em que $\|\mathbf{z}\|^{2}=\mathbf{z}^{\top} \mathbf{z}=(\mathbf{Y}-\mathbf{X} \boldsymbol{\beta})^{\top} \boldsymbol{\Sigma}^{-1 / 2} \boldsymbol{\Sigma}^{-1 / 2}(\mathbf{Y}-\mathbf{X} \boldsymbol{\beta})$. Para encontrarmos a matriz de informação esperada conforme mostrado por Lange et al. (1989), temos que

$$
\begin{aligned}
\mathbf{K}_{\beta_{k} \beta_{l}}(\boldsymbol{\beta}, \boldsymbol{\alpha})=\mathrm{E}\left(\frac{\partial l(\boldsymbol{\beta}, \boldsymbol{\alpha})}{\partial \beta_{k}} \frac{\partial l(\boldsymbol{\beta}, \boldsymbol{\alpha})}{\partial \beta_{l}}\right) & =4 \mathrm{E}\left(w_{g}^{2}(u) \mathbf{z}^{\top} \boldsymbol{\Sigma}^{-1 / 2} \mathbf{X}_{k} \mathbf{X}_{l}^{\top} \boldsymbol{\Sigma}^{-1 / 2} \mathbf{z}\right) \\
& =\frac{4}{m} \mathrm{E}\left(\|\mathbf{z}\|^{2} w_{g}^{2}(u)\right) \mathbf{X}_{l}^{\top} \boldsymbol{\Sigma}^{-1} \mathbf{X}_{k} .
\end{aligned}
$$


Então, a matriz de informação esperada avaliada no estimador de $\boldsymbol{\beta}$ fica dada por

$$
\mathbf{K}_{\beta \beta}\left(\hat{\boldsymbol{\beta}}_{\alpha}, \boldsymbol{\alpha}\right)=\left.\frac{4}{m} \mathrm{E}\left(\|\mathbf{z}\|^{2} w_{g}^{2}(u)\right)\right|_{\beta=\hat{\beta}_{\alpha}} \mathbf{X}^{\top} \boldsymbol{\Sigma}^{-1} \mathbf{X},
$$

em que $\|\mathbf{z}\|^{2}=u=(\mathbf{Y}-\mathbf{X} \boldsymbol{\beta})^{\top} \boldsymbol{\Sigma}^{-1}(\mathbf{Y}-\mathbf{X} \boldsymbol{\beta})$. Vemos que esta última expressão depende da função geradora, através de $w_{g}^{2}(u)$. Então, o ajuste da função de verossimilhança perfilada modificada aproximada para $\boldsymbol{\alpha}$ fica dado por

$$
\begin{aligned}
l_{p m a}^{*}(\boldsymbol{\alpha}) & =l_{p}(\boldsymbol{\alpha})-\frac{1}{2} \log \left|\mathbf{K}_{\beta \beta}\left(\hat{\boldsymbol{\beta}}_{\alpha}, \boldsymbol{\alpha}\right)\right| \\
& =l_{p}(\boldsymbol{\alpha})-\frac{1}{2} \log \left|\frac{4}{2 \pi m} \mathrm{E}\left(u w_{g}^{2}(u)\right)\right|_{\beta=\hat{\beta}_{\alpha}} \mathbf{X}^{\top} \boldsymbol{\Sigma}^{-1} \mathbf{X} \mid .
\end{aligned}
$$

Considerando a propriedade da decomposição do determinante de uma matriz ponderada por um escalar, isto é, $|a \mathbf{B}|=|a|^{p}|\mathbf{B}|$, em que $a$ é um escalar, $\mathbf{B}$ uma matriz $(p \times p)$ e $|a|$ é o valor absoluto de $a$, temos que a equação anterior fica dada por

$$
l_{p m a}^{*}(\boldsymbol{\alpha})=l_{p}(\boldsymbol{\alpha})-\frac{1}{2} \log \left|\mathbf{X}^{\top} \boldsymbol{\Sigma}^{-1} \mathbf{X}\right|-\frac{p}{2} \log \left|\frac{4}{2 \pi m} \mathrm{E}\left(u w_{g}^{2}(u)\right)\right|_{\beta=\hat{\beta}_{\alpha}} \mid,
$$

A derivada parcial de $l_{p m a}^{*}(\boldsymbol{\alpha})$ com respeito a $\alpha_{j}$ fica dada por

$$
\begin{aligned}
\frac{\partial}{\partial \alpha_{j}} l_{p m a}^{*}(\alpha)= & \frac{\partial}{\partial \alpha_{j}} l_{p}(\boldsymbol{\alpha})-\frac{1}{2} \frac{\partial}{\partial \alpha_{j}} \log \left|\mathbf{X}^{\top} \boldsymbol{\Sigma}^{-1} \mathbf{X}\right|-\frac{p}{2} \frac{\partial}{\partial \alpha_{j}} \log \left|\frac{4}{m} \mathrm{E}\left(\hat{u} w_{g}^{2}(\hat{u})\right)\right| \\
= & -\frac{1}{2} \operatorname{tr}\left\{\boldsymbol{\Sigma}^{-1} \frac{\partial \boldsymbol{\Sigma}}{\partial \alpha_{j}}\right\}+w_{g}\left(\hat{u}_{\alpha}\right)\left[\frac{\partial \hat{u}_{\alpha}}{\partial \alpha_{j}}\right]+\frac{1}{2} \operatorname{tr}\left\{\left(\mathbf{X}^{\top} \boldsymbol{\Sigma}^{-1} \mathbf{X}\right)^{-1} \mathbf{X}^{\top} \boldsymbol{\Sigma}^{-1} \frac{\partial \boldsymbol{\Sigma}}{\partial \alpha_{j}} \boldsymbol{\Sigma}^{-1} \mathbf{X}\right\} \\
& -\frac{p}{2} \frac{\partial}{\partial \alpha_{j}} \log \left|\frac{4}{m} \mathrm{E}\left(u w_{g}^{2}(u)\right)\right|_{\beta=\hat{\beta}_{\alpha}} \mid
\end{aligned}
$$

para $j=1, \ldots, q$. Para as distribuições elípticas em que a $\left.\mathrm{E}\left(u w_{g}^{2}(u)\right)\right|_{\beta=\hat{\beta}_{\alpha}}$ não depende de $\boldsymbol{\Sigma}$, o último termo da equação (3.22) será nulo, por exemplo, isso ocorre na distribuições normal, t-Student e exponencial potência. Então, a equação de estimação do ajuste da função de verossimilhança perfilada modificada aproximada com respeito a $\alpha_{j}$ ficará dada por

$$
\frac{\partial}{\partial \alpha_{j}} l_{p m a}^{*}(\alpha)=\frac{\partial}{\partial \alpha_{j}} l_{p}(\boldsymbol{\alpha})-\frac{1}{2} \frac{\partial}{\partial \alpha_{j}} \log \left|\mathbf{X}^{\top} \boldsymbol{\Sigma}^{-1} \mathbf{X}\right|
$$

para $j=1, \ldots, q$. Dado que o último termo não depende de $g(\cdot)$, a correção da função de verossimilhança perfilada modificada aproximada para estas distribuições elípticas será constante.

No Capítulo 2 não comparamos esta última aproximação da função de verossimilhança perfilada modificada visto que, para o caso particular da distribuição normal, as matrizes de informação observada e esperada avaliadas em $\hat{\boldsymbol{\beta}}_{\boldsymbol{\alpha}}$ coincidem. Desta forma, (3.18) e (3.21) também coincidem.

\section{Processo de estimação}

Da mesma forma que vimos no método de estimação restrito (ver Seção 3.3), o processo de estimação para os ajustes da verossimilhança perfilada tem dois passos. Primeiro obtemos uma estimativa para o vetor $\boldsymbol{\alpha}$ da maximização da equação (3.21) resolvendo a equação (3.22). Uma vez obtida a estimativa de $\boldsymbol{\Sigma}$, a estimativa de máxima verossimilhança perfilada modificada de $\boldsymbol{\beta}$ fica dada por

$$
\hat{\boldsymbol{\beta}}_{M V P M}=\left(\mathbf{X}^{\top} \widehat{\boldsymbol{\Sigma}}_{M V P M}^{-1} \mathbf{X}\right)^{-1} \mathbf{X}^{\top} \widehat{\boldsymbol{\Sigma}}_{M V P M}^{-1} \mathbf{Y} .
$$


Para a estimativa de máxima verossimilhança perfilada modificada aproximada o algoritmo tem a mesma forma.

Até agora vimos que as equações de estimação de $\alpha_{j}$ obtidas pelos métodos restrito, perfilada modificada e aproximada, tem termos similares à função escore de $\alpha_{j}$ da função de verossimilhança do modelo original e outros termos que dependem da transformação da variável $\mathbf{Y}$ e do cálculo da matriz de informação observada ou esperada, respectivamente. Não podemos concluir que obteremos as mesmas estimativas, como foi visto para o caso normal no capítulo anterior, em que as equações de estimação dos métodos coincidem. Na próxima seção analisaremos algumas estruturas da matriz $\boldsymbol{\Sigma}$ para comparar as equações de estimação dos métodos propostos.

\subsection{Casos particulares para a matriz de escala}

Em seguida, analisaremos algumas estruturas para a matriz de escala, para obter resultados que nos permitam comparar as expressões obtidas. Nas seções anteriores concluímos que a estrutura do estimador de $\boldsymbol{\beta}$ não depende da função $g(\cdot)$ mas que dependerá da estimativa de $\boldsymbol{\Sigma}$. Portanto, também mostraremos como fica a estimativa de $\boldsymbol{\beta}$ para cada estrutura de $\boldsymbol{\Sigma}$ que apresentemos.

\subsubsection{Estrutura da matriz escala simples}

Da Tabela 3.1 sabemos que o modelo normal pertence à classe elíptica e que a função geradora $g(u)$ para este caso é dada por $-\frac{1}{2} \exp (-u / 2)$. Vamos considerar a estrutura mais simples que foi apresentada na Seção 2.5 num modelo linear normal, em que a matriz $\boldsymbol{\Sigma}$ é dada por $\sigma^{2} \mathbf{I}_{m}$. Dos resultados do capítulo anterior temos que o estimador de $\boldsymbol{\beta}$, dado na equação (2.12), não depende de $\boldsymbol{\Sigma}$ e portanto é o mesmo para todos os métodos de estimação. Este estimador fica dado por

$$
\hat{\boldsymbol{\beta}}=\left(\mathbf{X}^{\top} \mathbf{X}\right)^{-1} \mathbf{X}^{\top} \mathbf{Y} .
$$

Para obter os diferentes estimadores de $\sigma^{2}$ consideraremos três funções da derivada parcial da matriz $\sigma^{2} \mathbf{I}_{m}$ com respeito a $\sigma^{2}$ descritas nas equações (B.8), (B.9) e (B.10) do Apêndice B.

O estimador de máxima verossimilhança para $\sigma^{2}$ será obtido a partir da equação (3.7), resultando em

$$
-\frac{m}{2 \sigma^{2}}-\frac{w_{g}(u)}{\left(\sigma^{2}\right)^{2}}(\mathbf{Y}-\mathbf{X} \boldsymbol{\beta})^{\top}(\mathbf{Y}-\mathbf{X} \boldsymbol{\beta})=0
$$

Podemos, portanto obter uma expressão para o estimador de máxima verossimilhança para $\sigma^{2}$ dado por

$$
\hat{\sigma}_{M V}^{2}=-\frac{2 w_{g}(u)}{m}(\mathbf{Y}-\mathbf{X} \hat{\boldsymbol{\beta}})^{\top}(\mathbf{Y}-\mathbf{X} \hat{\boldsymbol{\beta}}) .
$$

Vemos que esta última expressão depende da função geradora através de $w_{g}(u)$ (ver (3.6)), que pode não ser constante com respeito a $\sigma^{2}$. Portanto, não temos um estimador de máxima verossimilhança explícito para $\sigma^{2}$. Mas para o caso normal, $w_{g}(u)=-1 / 2$, sendo o caso mais simples em que o estimador de máxima verossimilhança é explícito para $\sigma^{2}$, mas depedente de $\boldsymbol{\beta}$.

Para o estimador de máxima verossimilhança restrita, de (3.16) obtemos a seguinte equação de estimação:

$$
\frac{\partial l\left(\sigma^{2} ; \mathbf{V}_{2}\right)}{\partial \sigma^{2}}=-\frac{m-p}{2 \sigma^{2}}-\frac{w_{2}\left(u_{2}\right)}{\sigma^{4}}(\mathbf{Y}-\mathbf{X b})^{\top}(\mathbf{Y}-\mathbf{X b})=0 .
$$

Para o caso normal, $w_{2}\left(u_{2}\right)=-1 / 2$ e o estimador resultante foi dado pela equação (2.13). 
Para obter o estimador de máxima verossimilhança perfilada modificada para $\sigma^{2}$, devemos considerar o estimador de máxima verossimilhança de $\boldsymbol{\beta}$ que neste caso não depende de $\sigma^{2}$, consideraremos $\hat{\boldsymbol{\beta}}_{\sigma}=\hat{\boldsymbol{\beta}}$, de (3.23). Da equação (3.19) temos que

$$
\frac{\partial}{\partial \sigma^{2}} l_{p m}^{*}\left(\boldsymbol{\beta}, \sigma^{2}\right)=-\frac{m-p}{2 \sigma^{2}}-\frac{C_{g}(\sigma)}{\sigma^{4}}(\mathbf{Y}-\mathbf{X} \hat{\boldsymbol{\beta}})^{\top}(\mathbf{Y}-\mathbf{X} \hat{\boldsymbol{\beta}})
$$

em que $C_{g}(\sigma)$ é uma função que depende da função geradora $g(\cdot)$ através das funções $w_{g}(\cdot)$ e $w_{g}^{\prime}(\cdot)$ avaliadas em $\hat{u}_{\sigma}$. A forma de $C_{g}(\sigma)$ foi especificada na equação (3.19) e portanto dependerá da distribuição elíptica que seja escolhida. Para o caso particular da distribuição normal temos que $w_{g}(\cdot)=-1 / 2$ e $w_{g}^{\prime}(\cdot)=0$, portanto $C_{g}(\sigma)=-1 / 2$ e o estimador de máxima verossimilhança perfilada modificada resultante ficou dado no capítulo anterior na equação (2.13).

Enquanto que a equação de estimação, usando máxima verossimilhança perfilada modificada aproximada de $\sigma^{2}$ num modelo linear elíptico, fica dada por

$$
\begin{aligned}
\frac{\partial}{\partial \sigma^{2}} l_{p m a}^{*}\left(\boldsymbol{\beta}, \sigma^{2}\right) & =-\frac{m}{2 \sigma^{2}}-\frac{w_{g}\left(\hat{u}_{\sigma}\right)}{\sigma^{4}}(\mathbf{Y}-\mathbf{X} \hat{\boldsymbol{\beta}})^{\top}(\mathbf{Y}-\mathbf{X} \hat{\boldsymbol{\beta}}) \\
& -\frac{p}{2} \frac{\partial}{\partial \sigma^{2}} \log \left|\frac{4}{m} \mathrm{E}\left\{u w_{g}^{2}(u)\right\}\right|_{\beta=\hat{\beta}} \mid-\frac{p}{2 \sigma^{2}}=0 .
\end{aligned}
$$

Como no caso normal temos que $w_{g}(u)=-1 / 2$ e a matriz de informação observada e esperada coincidem. Portanto, o estimador de máxima verossimilhança perfilada modificada e aproximada de $\sigma^{2}$, coincidem, sendo dados por

$$
\hat{\sigma}_{M V P M}^{2}=\hat{\sigma}_{M V P M A}^{2}=\frac{1}{m-p}(\mathbf{Y}-\mathbf{X} \hat{\boldsymbol{\beta}})^{\top}(\mathbf{Y}-\mathbf{X} \hat{\boldsymbol{\beta}}) .
$$

E assim, $\hat{\sigma}_{M V P M}^{2}=\hat{\sigma}_{M V P M A}^{2}=\hat{\sigma}_{M V R}^{2}$ para o caso normal.

Para a maioria das distribuições elípticas as funções $w_{g}(\cdot)$ e $w_{2}(\cdot)$ dependem dos parâmetros e dos dados. Portanto, não podemos concluir que a maximização das funções de verossimilhança perfilada modificada, perfilada modificada aproximada e restrita coincidem para todos os modelos lineares elípticos. Deste ponto de vista, o modelo normal torna-se o caso mais simples das distribuições elípticas.

Os resultados são similares quando considerarmos que a estrutura da matriz $\boldsymbol{\Sigma}$ é dada por $\sigma^{2} \boldsymbol{\Sigma}_{c}$, em que $\sigma^{2}$ é desconhecido e $\boldsymbol{\Sigma}_{c}$ é uma matriz simétrica, positiva definida e conhecida. De fato, na Seção B.3 do Apêndice B, em que apresentamos os cálculos das derivadas das matrizes para obter as equações de estimação de $\sigma^{2}$, a estrutura $\sigma^{2} \mathbf{I}_{m}$ é considerada um caso particular de $\sigma^{2} \boldsymbol{\Sigma}_{c}$. Assim, por exemplo, o estimador de $\boldsymbol{\beta}$ ficará dependendo de $\boldsymbol{\Sigma}_{c}$ mas não do parâmetro desconhecido $\sigma^{2}$ e é dado por

$$
\hat{\boldsymbol{\beta}}=\left(\mathbf{X}^{\top} \boldsymbol{\Sigma}_{c} \mathbf{X}\right)^{-1} \mathbf{X}^{\top} \boldsymbol{\Sigma}_{c} \mathbf{Y}
$$

Enquanto que os cálculos das equações de estimação de $\sigma^{2}$ podem ser obtidos da mesma forma que o caso visto nesta seção.

\subsubsection{Estrutura linear para a matriz escala}

Agora, um caso mais geral deste tipo de estruturas é considerar a matriz $\boldsymbol{\Sigma}$ como uma estrutura linear, isto é, $\boldsymbol{\Sigma}=\sum_{j=1}^{q} \alpha_{j} \mathbf{G}_{j}$, em que $q<m-p$. Para cada $j(\operatorname{com} j=1, \ldots, q)$ temos que $\alpha_{j}$ é um escalar desconhecido e a matriz $\mathbf{G}_{j}$ é particionada em blocos, contendo um matriz $\boldsymbol{\Sigma}_{j}$ na j-ésima posição da diagonal enquanto que os demais termos são zeros. A matriz $\mathbf{G}_{j}$ tem dimensão $(m \times m)$ e $\boldsymbol{\Sigma}_{j}$, conhecida, é simétrica e positiva definida de dimensão $\left(m_{j} \times m_{j}\right)$ para $j=1, \ldots, q$. 
A expressão para a matriz $\boldsymbol{\Sigma}$ é dada na equação (B.11) e a matriz $\mathbf{G}_{j}$ pode ser escrita na forma

$$
\mathbf{G}_{j}=\left[\begin{array}{cccccc}
\mathbf{0} & \ldots & \ldots & \ldots & \ldots & \ldots \\
\vdots & \mathbf{0} & \ldots & \ldots & \ldots & \ldots \\
\vdots & \vdots & \ddots & \ldots & \ldots & \ldots \\
\vdots & \vdots & \vdots & \boldsymbol{\Sigma}_{j} & \vdots & \vdots \\
\vdots & \vdots & \vdots & \ldots & \ddots & \vdots \\
\ldots & \ldots & \ldots & \ldots & \ldots & \mathbf{0}
\end{array}\right]
$$

Da mesma forma, consideremos as partições do vetor $\mathbf{Y}$ e da matriz $\mathbf{X}$ dadas por

$$
\mathbf{Y}=\left[\begin{array}{c}
\mathbf{Y}_{1} \\
\mathbf{Y}_{2} \\
\vdots \\
\mathbf{Y}_{j} \\
\vdots \\
\mathbf{Y}_{q}
\end{array}\right] \quad \mathbf{X}=\left[\begin{array}{c}
\mathbf{X}_{1} \\
\mathbf{X}_{2} \\
\vdots \\
\mathbf{X}_{j} \\
\vdots \\
\mathbf{X}_{q}
\end{array}\right]
$$

em que $\mathbf{Y}_{j}$ representa o $j$-ésimo vetor de dimensão $\left(m_{j} \times 1\right)$ da partição de $\mathbf{Y}$ e $\mathbf{X}_{j}$ representa a $j$-ésima matriz de dimensão $\left(m_{j} \times p\right)$ da partição de $\mathbf{X}$. A estrutura do estimador de $\boldsymbol{\beta}$ é a mesma para todos os métodos e ficará dependendo do estimador de $\boldsymbol{\Sigma}$. De modo geral, denotamos

$$
\hat{\boldsymbol{\beta}}_{\alpha}=\left(\mathbf{X}^{\top} \boldsymbol{\Sigma}^{-1} \mathbf{X}\right)^{-1} \mathbf{X}^{\top} \boldsymbol{\Sigma}^{-1} \mathbf{Y} .
$$

Para obter as equações de estimação de $\boldsymbol{\Sigma}$ consideremos os resultados apresentados nas equações (B.12), (B.13) e (B.14) da Seção B.3 do Apêndice B. O estimador de máxima verossimilhança para $\alpha_{j}$ será obtido fazendo a equação (3.7) igual a zero, ou seja

$$
-\alpha_{j}-\frac{2 w_{g}(u)}{m_{j}}\left(\mathbf{Y}_{j}-\mathbf{X}_{j} \boldsymbol{\beta}\right)^{\top} \boldsymbol{\Sigma}_{j}^{-1}\left(\mathbf{Y}_{j}-\mathbf{X}_{j} \boldsymbol{\beta}\right)=0
$$

para $j=1, \ldots, q$. Para o caso da distribuição normal a função $w_{g}(u)$ é igual a $-1 / 2$ e portanto o estimador de máxima verossimilhança para $\alpha_{j}$ é obtido de

$$
\alpha_{j_{M V}}=\frac{1}{m_{j}}\left(\mathbf{Y}_{j}-\mathbf{X}_{j} \hat{\boldsymbol{\beta}}\right)^{\top} \boldsymbol{\Sigma}_{j}^{-1}\left(\mathbf{Y}_{j}-\mathbf{X}_{j} \hat{\boldsymbol{\beta}}\right),
$$

para $j=1, \ldots, q$.

Da equação (3.16) temos para $\alpha_{j}$ a equação de estimação da função de verosimilhança restrita $-\alpha_{j} m_{j}+\operatorname{tr}\left\{\left(\sum_{i=1}^{q} \frac{1}{\alpha_{i}} \mathbf{X}_{i}^{\top} \mathbf{G}_{i}^{-1} \mathbf{X}_{i}\right)^{-1} \mathbf{X}_{j}^{\top} \mathbf{G}_{j}^{-1} \mathbf{X}_{j}\right\}-2 w_{2}\left(u_{2}\right)\left(\mathbf{Y}_{j}-\mathbf{X}_{j} \mathbf{b}\right)^{\top} \boldsymbol{\Sigma}_{j}^{-1}\left(\mathbf{Y}_{j}-\mathbf{X}_{j} \mathbf{b}\right)=0$, para $j=1, \ldots, q$. Para o caso normal não temos uma expressão explícita mas podemos escrever uma função do estimador como

$$
\alpha_{j_{M V R}}=\frac{1}{m_{j}}\left(\mathbf{Y}_{j}-\mathbf{X}_{j} \mathbf{b}\right)^{\top} \boldsymbol{\Sigma}_{j}^{-1}\left(\mathbf{Y}_{j}-\mathbf{X}_{j} \mathbf{b}\right)+\frac{1}{m_{j}} \operatorname{tr}\left\{\left(\sum_{i=1}^{q} \frac{1}{\alpha_{i}} \mathbf{X}_{i}^{\top} \mathbf{G}_{i}^{-1} \mathbf{X}_{i}\right)^{-1} \mathbf{X}_{j}^{\top} \mathbf{G}_{j}^{-1} \mathbf{X}_{j}\right\},
$$

para $j=1, \ldots, q$.

Para a equação de estimação de máxima verossimilhança perfilada modificada para $\alpha_{j}$ utilizamos 
o resultado da equação (3.19) e consideramos a derivada parcial de $\hat{u}_{\alpha}$ com respeito a $\alpha_{j}$. Assim obtemos

$-\alpha_{j}+\frac{1}{m_{j}} \operatorname{tr}\left\{\left(\sum_{i=1}^{q} \frac{1}{\alpha_{i}} \mathbf{X}_{i}^{\top} \mathbf{G}_{i}^{-1} \mathbf{X}_{i}\right)^{-1} \mathbf{X}_{j}^{\top} \mathbf{G}_{j}^{-1} \mathbf{X}_{j}\right\}-\frac{2 C_{g}(\boldsymbol{\alpha})}{m_{j}}\left(\mathbf{Y}_{j}-\mathbf{X}_{j} \hat{\boldsymbol{\beta}}\right)^{\top} \boldsymbol{\Sigma}_{j}^{-1}\left(\mathbf{Y}_{j}-\mathbf{X}_{j} \hat{\boldsymbol{\beta}}\right)=0$

em que $C_{g}(\boldsymbol{\alpha})$ é dado por $\left(2 w_{g}\left(\hat{u}_{\alpha}\right)^{2}-p w_{g}^{\prime}\left(\hat{u}_{\alpha}\right)\right) / 2 w_{g}\left(\hat{u}_{\alpha}\right)$. No caso normal, $C_{g}(\boldsymbol{\alpha})=-1 / 2$, assim podemos escrever uma função do estimador na forma

$\alpha_{j_{M V P M}}=\frac{1}{m_{j}}\left(\mathbf{Y}_{j}-\mathbf{X}_{j} \hat{\boldsymbol{\beta}}\right)^{\top} \boldsymbol{\Sigma}_{j}^{-1}\left(\mathbf{Y}_{j}-\mathbf{X}_{j} \hat{\boldsymbol{\beta}}\right)+\frac{1}{m_{j}} \operatorname{tr}\left\{\left(\sum_{i=1}^{q} \frac{1}{\alpha_{i}} \mathbf{X}_{i}^{\top} \mathbf{G}_{i}^{-1} \mathbf{X}_{i}\right)^{-1} \mathbf{X}_{j}^{\top} \mathbf{G}_{j}^{-1} \mathbf{X}_{j}\right\}$

para $j=1, \ldots, q$.

Por último a equação de estimação de máxima verossimilhança perfilada modificada aproximada para $\alpha_{j}$ fica dada por

$$
\begin{array}{r}
-\frac{m_{j}}{2 \alpha_{j}}+\frac{1}{2 \alpha_{j}^{2}} \operatorname{tr}\left\{\left(\sum_{i=1}^{q} \frac{1}{\alpha_{i}} \mathbf{X}_{i}^{\top} \mathbf{G}_{i}^{-1} \mathbf{X}_{i}\right)^{-1} \mathbf{X}_{j}^{\top} \mathbf{G}_{j}^{-1} \mathbf{X}_{j}\right\}-\frac{w_{g}\left(\hat{u}_{\alpha}\right)}{\alpha_{j}^{2}}\left(\mathbf{Y}_{j}-\mathbf{X}_{j} \hat{\boldsymbol{\beta}}\right)^{\top} \boldsymbol{\Sigma}_{j}^{-1}\left(\mathbf{Y}_{j}-\mathbf{X}_{j} \hat{\boldsymbol{\beta}}\right) \\
-\frac{p}{2} \frac{\partial}{\partial \alpha_{j}} \log \left|\frac{4}{m} \mathrm{E}\left(u w_{g}^{2}(u)\right)\right|_{\beta=\hat{\beta}_{\alpha}} \mid=0
\end{array}
$$

para $j=1, \ldots, q$. Para o caso normal temos que $w_{g}^{2}(u)=1 / 4$ e portanto $\left.\mathrm{E}\left(u w_{g}^{2}(u)\right)\right|_{\beta=\hat{\beta}_{\alpha}}=$ $(1 / 4)(m-p)$ sendo constante com respeito a $\alpha_{j}$. Assim, uma expressão resultante para o estimador fica dada por

$\alpha_{j M V P M A}=\frac{1}{m_{j}}\left(\mathbf{Y}_{j}-\mathbf{X}_{j} \hat{\boldsymbol{\beta}}\right)^{\top} \boldsymbol{\Sigma}_{j}^{-1}\left(\mathbf{Y}_{j}-\mathbf{X}_{j} \hat{\boldsymbol{\beta}}\right)+\frac{1}{m_{j}} \operatorname{tr}\left\{\left(\sum_{i=1}^{q} \frac{1}{\alpha_{i}} \mathbf{X}_{i}^{\top} \mathbf{G}_{i}^{-1} \mathbf{X}_{i}\right)^{-1} \mathbf{X}_{j}^{\top} \mathbf{G}_{j}^{-1} \mathbf{X}_{j}\right\}$,

para $j=1, \ldots, q$.

Vemos que as equações de estimação da verossimilhança restrita, verossimilhança perfilada modificada e verossimilhança perfilada modificada aproximada para $\alpha_{j}$ são as mesmas para o caso normal, mas não temos uma expressão explícita para o estimador.

\subsubsection{Outro tipo de estrutura linear com dois parâmetros}

Outra estrutura de $\boldsymbol{\Sigma}$ que podemos considerar é dada por $\left(\sigma^{2} \mathbf{I}_{m}+\tau \mathbf{J}_{m}\right)$ em que $\mathbf{J}_{m}$ é uma matriz de uns e que pode ser escrita como $\mathbf{1 1}^{\top}$, sendo $\mathbf{1}$ um vetor de uns de dimensão $(m \times 1)$. A expressão da matriz $\boldsymbol{\Sigma}$ é dada na equação (B.15). Para obter as equações de estimação do vetor $\boldsymbol{\alpha}=\left(\sigma^{2}, \tau\right)$ consideramos que o estimador de $\boldsymbol{\beta}$ tem a mesma estrutura apresentada nos casos anteriores e que utilizaremos os resultados do item 3 da Seção B.3 do Apêndice B. Especificamente as equações (B.16), (B.17) e (B.18) para $\sigma^{2}$ e as equações (B.19), (B.20) e (B.21) para $\tau$. As equações de estimação de máxima verossimilhança para $\sigma^{2}$ e $\tau$ são obtidas a partir da equação (3.7) e são dadas por

$$
\begin{aligned}
& \frac{\partial}{\partial \sigma^{2}} l(\boldsymbol{\beta}, \boldsymbol{\alpha} ; \mathbf{Y})=0, \\
& -\frac{1}{2} \frac{m}{\sigma^{2}}\left\{\frac{\sigma^{2}+(m-1) \tau}{\sigma^{2}+m \tau}\right\}-w_{g}(u)\left[\frac{1}{\sigma^{2}} S_{Y Y}-\frac{\left(2 \tau \sigma^{2}+m \tau^{2}\right)}{\left\{\sigma^{2}\left(\sigma^{2}+m \tau\right)\right\}^{2}} S_{Y}^{2}\right]=0
\end{aligned}
$$


e

$$
\begin{aligned}
& \frac{\partial}{\partial \tau} l(\boldsymbol{\beta}, \boldsymbol{\alpha} ; \mathbf{Y})=0 \\
& -\frac{1}{2} \frac{m}{\sigma^{2}+m \tau}-w_{g}(u)\left(\frac{1}{\sigma^{2}+m \tau}\right)^{2} S_{Y}^{2}=0
\end{aligned}
$$

em que $S_{Y Y}=\sum_{i=1}^{m}\left(Y_{i}-\mathbf{X}_{i}^{\top} \boldsymbol{\beta}\right)^{2}$ e $S_{Y}^{2}=\left(\sum_{i=1}^{m}\left(Y_{i}-\mathbf{X}_{i}^{\top} \boldsymbol{\beta}\right)\right)^{2}$. Para o caso normal, em que $w_{g}(u)=-1 / 2$, a equação e estimação para $\sigma^{2}$ fica dada por

$$
-\frac{1}{2} \frac{m}{\sigma^{2}}\left\{\frac{\sigma^{2}+(m-1) \tau}{\sigma^{2}+m \tau}\right\}+\frac{1}{2}\left[\frac{1}{\sigma^{2}} S_{Y Y}-\frac{\left(2 \tau \sigma^{2}+m \tau^{2}\right)}{\left\{\sigma^{2}\left(\sigma^{2}+m \tau\right)\right\}^{2}} S_{Y}^{2}\right]=0 .
$$

Enquanto que para $\tau$ obtemos

$$
-\frac{1}{2} \frac{m}{\sigma^{2}+m \tau}+\frac{1}{2}\left(\frac{1}{\sigma^{2}+m \tau}\right)^{2} S_{Y}^{2}=0
$$

então,

$$
\tau_{M V}=\frac{S_{y}^{2}-m \hat{\sigma}^{2}}{m^{2}}
$$

Não temos uma expressão explícita para $\sigma^{2}$ e a expressão do estimador de $\tau$ fica dependendo do estimador de $\sigma^{2}$.

A equação de estimação de máxima verossimilhança restrita (3.16) de $\sigma^{2}$ fica dada por

$$
\begin{aligned}
& \frac{1}{2\left(\sigma^{2}\right)\left(\sigma^{2}+m \tau\right)} \operatorname{tr}\left[\left\{\left(\sigma^{2}+m \tau\right) \mathbf{X}^{\top} \mathbf{X}-\tau \mathbf{X}^{\top} \mathbf{J}_{m} \mathbf{X}\right\}^{-1}\left\{\left(\sigma^{2}+m \tau\right)^{2} \mathbf{X}^{\top} \mathbf{X}-\left(2 \tau \sigma^{2}+m \tau^{2}\right) \mathbf{X}^{\top} \mathbf{J}_{m} \mathbf{X}^{2}\right]\right. \\
& -\frac{1}{2} \frac{m}{\sigma^{2}}\left\{\frac{\sigma^{2}+(m-1) \tau}{\sigma^{2}+m \tau}\right\}-w_{2}\left(u_{2}\right)\left[\frac{1}{\sigma^{2}} S_{Y Y}-\frac{\left(2 \tau \sigma^{2}+m \tau^{2}\right)}{\left\{\sigma^{2}\left(\sigma^{2}+m \tau\right)\right\}^{2}} S_{Y}^{2}\right]=0 .
\end{aligned}
$$

Vemos que apenas o último termo depende da função geradora de densidade das distribuições elípticas, isto é, que os primeiros dois termos serão constante para todas as distribuições elípticas. Enquanto que para $\tau$ a equação de estimação fica dada por $\frac{\sigma^{2}}{2\left(\sigma^{2}+m \tau\right)} \operatorname{tr}\left[\left\{\left(\sigma^{2}+m \tau\right) \mathbf{X}^{\top} \mathbf{X}-\tau \mathbf{X}^{\top} \mathbf{J}_{m} \mathbf{X}\right\}^{-1} \mathbf{X}^{\top} \mathbf{J}_{m} \mathbf{X}\right]-\frac{1}{2} \frac{m}{\sigma^{2}+m \tau}-w_{2}\left(u_{2}\right)\left(\frac{1}{\sigma^{2}+m \tau}\right)^{2} S_{Y}^{2}=0$.

Para o caso normal, em que $w_{2}\left(u_{2}\right)=-1 / 2$, a equação de estimação para $\sigma^{2}$ fica dada por

$$
\begin{aligned}
& \frac{1}{2\left(\sigma^{2}\right)\left(\sigma^{2}+m \tau\right)} \operatorname{tr}\left[\left\{\left(\sigma^{2}+m \tau\right) \mathbf{X}^{\top} \mathbf{X}-\tau \mathbf{X}^{\top} \mathbf{J}_{m} \mathbf{X}\right\}^{-1}\left\{\left(\sigma^{2}+m \tau\right)^{2} \mathbf{X}^{\top} \mathbf{X}-\left(2 \tau \sigma^{2}+m \tau^{2}\right) \mathbf{X}^{\top} \mathbf{J}_{m} \mathbf{X}^{2}\right\}\right] \\
& -\frac{1}{2} \frac{m}{\sigma^{2}}\left\{\frac{\sigma^{2}+(m-1) \tau}{\sigma^{2}+m \tau}\right\}+\frac{1}{2}\left[\frac{1}{\sigma^{2}} S_{Y Y}-\frac{\left(2 \tau \sigma^{2}+m \tau^{2}\right)}{\left\{\sigma^{2}\left(\sigma^{2}+m \tau\right)\right\}^{2}} S_{Y}^{2}\right]=0 .
\end{aligned}
$$

Diferentemente da estimação de máxima verossimilhança para $\tau$, aqui não temos uma expressão explícita para o estimador de máxima verossimilhança restrita pois a equação resultante fica de- 
pendendo de uma função do traço dada por

$\frac{\sigma^{2}}{2\left(\sigma^{2}+m \tau\right)} \operatorname{tr}\left[\left\{\left(\sigma^{2}+m \tau\right) \mathbf{X}^{\top} \mathbf{X}-\tau \mathbf{X}^{\top} \mathbf{J}_{m} \mathbf{X}\right\}^{-1} \mathbf{X}^{\top} \mathbf{J}_{m} \mathbf{X}\right]-\frac{1}{2} \frac{m}{\sigma^{2}+m \tau}+\frac{1}{2}\left(\frac{1}{\sigma^{2}+m \tau}\right)^{2} S_{Y}^{2}=0$.

De forma comparativa podemos escrever uma função do estimador de máxima verossimilhança restrita de $\tau$ como

$$
\tau_{M V R}=\frac{\left.S_{y}^{2}-\left\{m-\hat{\sigma}^{2} \operatorname{tr}\left(a r_{\tau}\right)\right) \hat{\sigma}^{2}\right\}}{m\left\{m-\hat{\sigma}^{2} \operatorname{tr}\left(a r_{\tau}\right)\right\}}
$$

em que o argumento $a r_{\tau}$ é função de $\tau$ e é dado por $\left\{\left(\sigma^{2}+m \tau\right) \mathbf{X}^{\top} \mathbf{X}-\tau \mathbf{X}^{\top} \mathbf{J}_{m} \mathbf{X}\right\}^{-1} \mathbf{X}^{\top} \mathbf{J}_{m} \mathbf{X}$. Considerando a função de verossimilhança perfilada temos que a equação de estimação de máxima verossimilhança perfilada modificada para $\sigma^{2}$ é dada por

$$
\begin{array}{r}
\frac{1}{2 \sigma^{2}\left(\sigma^{2}+m \tau\right)} \operatorname{tr}\left[\left\{\left(\sigma^{2}+m \tau\right) \mathbf{X}^{\top} \mathbf{X}-\tau \mathbf{X}^{\top} \mathbf{J}_{m} \mathbf{X}\right\}^{-1}\left\{\left(\sigma^{2}+m \tau\right)^{2} \mathbf{X}^{\top} \mathbf{X}-\left(2 \tau \sigma^{2}+m \tau^{2}\right) \mathbf{X}^{\top} \mathbf{J}_{m} \mathbf{X}\right\}\right] \\
-\frac{m}{\sigma^{2}}\left\{\frac{\sigma^{2}+(m-1) \tau}{\sigma^{2}+m \tau}\right\}-C_{g}(\boldsymbol{\alpha})\left[\frac{1}{\sigma^{2}} S_{Y Y}-\frac{\left(2 \tau \sigma^{2}+m \tau^{2}\right)}{\left\{\sigma^{2}\left(\sigma^{2}+m \tau\right)\right\}^{2}} S_{Y}^{2}\right]=0 .
\end{array}
$$

Enquanto que para $\tau$ temos que

$$
\frac{\sigma^{2}}{2\left(\sigma^{2}+m \tau\right)} \operatorname{tr}\left[\left\{\left(\sigma^{2}+m \tau\right) \mathbf{X}^{\top} \mathbf{X}-\tau \mathbf{X}^{\top} \mathbf{J}_{m} \mathbf{X}\right\}^{-1} \mathbf{X}^{\top} \mathbf{J}_{m} \mathbf{X}\right]-\frac{1}{2} \frac{m}{\sigma^{2}+m \tau}-C_{g}(\boldsymbol{\alpha})\left(\frac{1}{\sigma^{2}+m \tau}\right)^{2} S_{Y}^{2}=0 .
$$

Lembrando que o termo $C_{g}(\boldsymbol{\alpha})$ é uma função que depende do vetor de parâmetros $\boldsymbol{\alpha}$ e da função geradora de densidade $g(\cdot)$. Para o caso normal $C_{g}(\boldsymbol{\alpha})$ é igual à $-1 / 2$, então as equações coincidem com as equações resultantes para a estimação restrita (3.25) e (3.26).

Por último, as equações de estimação para encontrar os estimadores de máxima verossimilhança perfilada modificada aproximada ficam, respectivamente, dadas por

$$
\begin{gathered}
\frac{1}{2 \sigma^{2}\left(\sigma^{2}+m \tau\right)} \operatorname{tr}\left[\left\{\left(\sigma^{2}+m \tau\right) \mathbf{X}^{\top} \mathbf{X}-\tau \mathbf{X}^{\top} \mathbf{J}_{m} \mathbf{X}\right\}^{-1}\left\{\left(\sigma^{2}+m \tau\right)^{2} \mathbf{X}^{\top} \mathbf{X}-\left(2 \tau \sigma^{2}+m \tau^{2}\right) \mathbf{X}^{\top} \mathbf{J}_{m} \mathbf{X}\right\}\right] \\
-\frac{m}{\sigma^{2}}\left\{\frac{\sigma^{2}+(m-1) \tau}{\sigma^{2}+m \tau}\right\}+w_{g}\left(\hat{u}_{\alpha}\right)\left[\frac{1}{\sigma^{2}} S_{Y Y}-\frac{\left(2 \tau \sigma^{2}+m \tau^{2}\right)}{\left\{\sigma^{2}\left(\sigma^{2}+m \tau\right)\right\}^{2}} S_{Y}^{2}\right]-\frac{p}{2} \frac{\partial}{\partial \sigma^{2}} \log \left|\frac{4}{m} \mathrm{E}\left(u w_{g}^{2}(u)\right)\right|_{\beta=\hat{\beta}_{\alpha}} \mid=0
\end{gathered}
$$

$\mathrm{e}$

$$
\begin{array}{r}
\frac{\sigma^{2}}{2\left(\sigma^{2}+m \tau\right)} \operatorname{tr}\left[\left\{\left(\sigma^{2}+m \tau\right) \mathbf{X}^{\top} \mathbf{X}-\tau \mathbf{X}^{\top} \mathbf{J}_{m} \mathbf{X}\right\}^{-1} \mathbf{X}^{\top} \mathbf{J}_{m} \mathbf{X}\right]-\frac{1}{2} \frac{m}{\sigma^{2}+m \tau}+w_{g}\left(\hat{u}_{\alpha}\right)\left(\frac{1}{\sigma^{2}+m \tau}\right)^{2} S_{Y}^{2} \\
-\frac{p}{2} \frac{\partial}{\partial \tau} \log \left|\frac{4}{m} \mathrm{E}\left(u w_{g}^{2}(u)\right)\right|_{\beta=\hat{\beta}_{\alpha}} \mid=0 .
\end{array}
$$

Lembrando que no caso normal os métodos de perfilada modificada e perfilada modificada aproximada coincidem pois a matriz de informação observada e esperada são as mesmas. 


\subsubsection{Estrutura para a matriz escala num modelo autoregressivo}

Uma última estrutura que analisaremos é considerar um modelo autoregressivo de ordem 1, em que a matriz $\boldsymbol{\Sigma}$ é dada por $\sigma^{2} \mathbf{R}$ e $\mathbf{R}$ escrita como

$$
\mathbf{R}=\left[\begin{array}{cccccc}
1 & \rho & \rho^{2} & \ldots & \rho^{m-2} & \rho^{m-1} \\
\rho & 1 & \rho & \ldots & \rho^{m-3} & \rho^{m-2} \\
\rho^{2} & \rho & 1 & \ldots & \rho^{m-4} & \rho^{m-3} \\
\vdots & \vdots & \vdots & \ddots & \vdots & \vdots \\
\rho^{m-2} & \rho^{m-3} & \rho^{m-4} & \ldots & 1 & \rho \\
\rho^{m-1} & \rho^{m-2} & \rho^{m-3} & \ldots & \rho & 1
\end{array}\right]
$$

Assim, o vetor de parâmetros $\boldsymbol{\alpha}$ fica dado por $\left(\sigma^{2}, \rho\right)$. A matriz $\mathbf{X}$ para este modelo é um vetor de uns de dimensão $(m \times 1)$. A estrutura do estimador de $\beta$, um escalar, é a mesma para todos os métodos e ficará dependendo do estimador de $\boldsymbol{\Sigma}$. De modo geral denotamos

$$
\hat{\beta}_{\alpha}=\left(\mathbf{X}^{\top} \boldsymbol{\Sigma}^{-1} \mathbf{X}\right)^{-1} \mathbf{X}^{\top} \boldsymbol{\Sigma}^{-1} \mathbf{Y} .
$$

Considerando as equações (B.26) e (B.27) da Seção B.3 do Apêndice B temos que a forma do estimador de $\beta$ fica dada por

$$
\begin{aligned}
\left(\mathbf{X}^{\top} \boldsymbol{\Sigma}^{-1} \mathbf{X}\right)^{-1} \mathbf{X}^{\top} \boldsymbol{\Sigma}^{-1} \mathbf{Y} & =\left(\mathbf{X}^{\top} \frac{1}{\sigma^{2}} \mathbf{R}^{-1} \mathbf{X}\right)^{-1} \mathbf{X}^{\top} \frac{1}{\sigma^{2}} \mathbf{R}^{-1} \mathbf{Y}=\left(\mathbf{X}^{\top} \mathbf{R}^{-1} \mathbf{X}\right)^{-1} \mathbf{X}^{\top} \mathbf{R}^{-1} \mathbf{Y} \\
& =\frac{\sum_{i=1}^{m} Y_{i}-\rho \sum_{i=2}^{m-1} Y_{i}}{m-\rho(m-2)}
\end{aligned}
$$

Para determinar os estimadores do parâmetro $\sigma^{2}$ vamos considerar que a matriz $\mathbf{R}$ não depende deste parâmetro e portanto as equações de estimação são similares às obtidas no primeiro modelo apresentado neste capítulo. Enquanto que para $\rho$ vamos considerar as equações de estimação para $\alpha_{j}$ dadas nas Seções 3.2, 3.3 e 3.4 e os resultados obtidos nas equações (B.22), (B.28) e (B.29) da Seção B.3 do Apêndice B. Assim, para o estimador de máxima verossimilhança para $\rho$ temos que a equação de estimação fica dada por

$\frac{\partial}{\partial \rho} l(\boldsymbol{\beta}, \boldsymbol{\alpha})=\frac{-2 \rho(m-1)}{2\left(1-\rho^{2}\right)}-\frac{w_{g}(u)}{\left(1-\rho^{2}\right)^{2}}\left[(1-\rho)^{2}\left\{\left(Y_{1}-\beta\right)^{2}+\left(Y_{m}-\beta\right)^{2}\right\}+2(1+\rho)^{2} \sum_{i=2}^{m-1}\left(Y_{i}-\beta\right)^{2}\right]=0$.

Para o caso da distribuição normal a função $w_{g}(u)$ é igual a $-1 / 2$ e portanto a equação de estimação de máxima verossimilhança para $\rho$ fica dado por

$$
\frac{-2 \rho(m-1)}{2\left(1-\rho^{2}\right)}+\frac{1}{2\left(1-\rho^{2}\right)^{2}}\left[(1-\rho)^{2}\left\{\left(Y_{1}-\beta\right)^{2}+\left(Y_{m}-\beta\right)^{2}\right\}+2(1+\rho)^{2} \sum_{i=2}^{m-1}\left(Y_{i}-\beta\right)^{2}\right]=0 .
$$

Considerando a equação (3.16) a equação de estimação da função de verosimilhança restrita fica dada por

$$
\begin{aligned}
-\rho(m-1)\left(1-\rho^{2}\right)-w_{2}\left(u_{2}\right)\left[(1-\rho)^{2}\left\{\left(Y_{1}-\beta\right)^{2}+\left(Y_{m}-\beta\right)^{2}\right\}+2(1+\rho)^{2} \sum_{i=2}^{m-1}\left(Y_{i}-\beta\right)^{2}\right] & \\
+\frac{(1-\rho)\left(1-\rho^{2}\right)+(m-2)(1+\rho)^{3}}{(m-(m-2) \rho)} & =0 .
\end{aligned}
$$


Para o caso normal não temos uma expressão explícita mas podemos escrever uma função do estimador como

$$
\begin{aligned}
-\rho(m-1)\left(1-\rho^{2}\right)+\frac{1}{2}\left[(1-\rho)^{2}\left\{\left(Y_{1}-\beta\right)^{2}+\left(Y_{m}-\beta\right)^{2}\right\}+2(1+\rho)^{2} \sum_{i=2}^{m-1}\left(Y_{i}-\beta\right)^{2}\right] & \\
+\frac{(1-\rho)\left(1-\rho^{2}\right)+(m-2)(1+\rho)^{3}}{(m-(m-2) \rho)} & =0 .
\end{aligned}
$$

Para a equação de estimação de máxima verossimilhança perfilada modificada para $\rho$ utilizamos o resultado da equação (3.19) e consideramos a derivada parcial de $\hat{u}_{\rho}$ com respeito a $\rho$. Assim, obtemos

$$
\begin{aligned}
-\rho(m-1)\left(1-\rho^{2}\right)-C_{g}(\boldsymbol{\alpha})\left[(1-\rho)^{2}\left\{\left(Y_{1}-\beta\right)^{2}+\left(Y_{m}-\beta\right)^{2}\right\}+2(1+\rho)^{2} \sum_{i=2}^{m-1}\left(Y_{i}-\beta\right)^{2}\right] & \\
+\frac{(1-\rho)\left(1-\rho^{2}\right)+(m-2)(1+\rho)^{3}}{(m-(m-2) \rho)} & =0
\end{aligned}
$$

em que $C_{g}(\boldsymbol{\alpha})$ é dado por $\left(2 w_{g}\left(\hat{u}_{\alpha}\right)^{2}-p w_{g}^{\prime}\left(\hat{u}_{\alpha}\right)\right) / 2 w_{g}\left(\hat{u}_{\alpha}\right)$. No caso normal, devemos substituir $C_{g}(\boldsymbol{\alpha})=-1 / 2$, obtendo um resultado igual à equação (3.28) Por último, a equação de estimação de máxima verossimilhança perfilada modificada aproximada para $\alpha_{j}$ fica dada por

$$
\begin{aligned}
-\rho(m-1)\left(1-\rho^{2}\right)+ & w_{g}\left(\hat{u}_{\alpha}\right)\left[(1-\rho)^{2}\left\{\left(Y_{1}-\beta\right)^{2}+\left(Y_{m}-\beta\right)^{2}\right\}+2(1+\rho)^{2} \sum_{i=2}^{m-1}\left(Y_{i}-\beta\right)^{2}\right] \\
& +\frac{(1-\rho)\left(1-\rho^{2}\right)+(m-2)(1+\rho)^{3}}{(m-(m-2) \rho)}-\frac{p}{2} \frac{\partial}{\partial \rho} \log \left|\frac{4}{m} \mathrm{E}\left(u w_{g}^{2}(u)\right)\right|_{\beta=\hat{\beta}_{\alpha}} \mid=0 .
\end{aligned}
$$

Para o caso normal temos que $w_{g}^{2}(u)=1 / 4$ e portanto $\left.\mathrm{E}\left\{u w_{g}^{2}(u)\right\}\right|_{\beta=\hat{\beta}_{\alpha}}=(1 / 4)(m-p)$ sendo constante com respeito a $\alpha_{j}$. Assim, uma expressão resultante para o estimador fica dada por

$$
\begin{aligned}
-\rho(m-1)\left(1-\rho^{2}\right)+\frac{1}{2}\left[(1-\rho)^{2}\left\{\left(Y_{1}-\beta\right)^{2}+\left(Y_{m}-\beta\right)^{2}\right\}+2(1+\rho)^{2} \sum_{i=2}^{m-1}\left(Y_{i}-\beta\right)^{2}\right] & \\
+\frac{(1-\rho)\left(1-\rho^{2}\right)+(m-2)(1+\rho)^{3}}{(m-(m-2) \rho)} & =0 .
\end{aligned}
$$

Vemos que as equações de estimação de verossimilhança restrita, verossimilhança perfilada modificada e verossimilhança perfilada modificada aproximada para $\rho$ são as mesmas para o caso normal, mas não temos uma expressão explícita para o estimador.

Na seção seguinte discutiremos alguns casos particulares de distribuições elípticas, de tal maneira que especificando a função geradora de densidade $g(\cdot)$ podemos comparar as expressões dos estimadores resultantes.

\subsection{Casos particulares de distribuições elípticas}

Nesta seção resumiremos a aplicação dos três métodos de estimação considerados neste trabalho para algumas distribuições elípticas. Na Tabela 3.1 apresentamos as distribuições elípticas mais utilizadas. Deste modo, precisaremos da especificação da função $w_{g}(\cdot)$, definida em (3.6), o resumo é apresentado na Tabela 3.2, em que também especificamos $w_{g}^{\prime}(\cdot)$ para algumas distribuições. 
Tabela 3.2: Expressões de $w_{g}(u)$ e $w_{g}^{\prime}(u)$ para algumas distribuições pertencentes à família elíptica.

\begin{tabular}{|l|c|c|}
\hline Distribuição & $w_{g}(u)$ & $w_{g}^{\prime}(u)$ \\
\hline Normal & $-1 / 2$ & 0 \\
t-Student & $-\{(\nu+m) /(2 \nu)\}(1+u / \nu)^{-1}$ & $\left\{(\nu+m) /\left(2 \nu^{2}\right)\right\}(1+u / \nu)^{-2}$ \\
$\begin{array}{l}\text { Exponencial } \\
\text { potência }\end{array}$ & $(-1 / 2) \lambda u^{\lambda-1}$ & $(-1 / 2) \lambda(\lambda-1) u^{\lambda-2}$ \\
\hline
\end{tabular}

Nas Seções 3.2, 3.3 e 3.4, vimos que estimador de $\boldsymbol{\beta}$ obtido pelos três métodos de estimação e para todas as distribuições elípticas têm a mesma estrutura. Então, nesta seção não voltaremos a obter o estimador, apenas notamos que sua forma é dada por $\hat{\boldsymbol{\beta}}=\left(\mathbf{X}^{\top} \boldsymbol{\Sigma}^{-1} \mathbf{X}\right)^{-1} \mathbf{X}^{\top} \boldsymbol{\Sigma}^{-1} \mathbf{Y}$ e sua estimativa dependerá do método escolhido para estimar a estrutura de $\boldsymbol{\Sigma}$.

\subsubsection{Modelo linear t-Student}

$\mathrm{O}$ modelo linear t-Student, pertence à classe de modelos lineares com erros elípticos. Então, consideramos que o vetor $\mathbf{Y}$ segue uma distribuição t-Student multivariada com parâmetros $\boldsymbol{\beta}, \boldsymbol{\Sigma}$ e $\nu$, em que $\nu$ representa os graus de liberdade que assumiremos conhecido. Assim, nosso principal objetivo será a estimação de $\boldsymbol{\beta}$ e $\boldsymbol{\Sigma}$. Da Tabela 3.1 temos que a função $g(u)$ será igual a $c_{2}(1+$ $u / \nu)^{-(\nu+m) / 2}$, com $c_{2}$ dado em (3.2).

\section{Estrutura do modelo}

Seja Y um vetor aleatório com distribuição t-Student e parâmetros $\boldsymbol{\mu}_{(m \times 1)}, \Sigma_{(m \times m)}$ e $\nu$ graus de liberdade, que denotaremos como

$$
\mathbf{Y} \sim t_{m}(\boldsymbol{\mu}, \boldsymbol{\Sigma}, \nu)
$$

Lembremos que a variável $u$ é a forma quadrática $(\mathbf{Y}-\boldsymbol{\mu})^{\top} \boldsymbol{\Sigma}^{-1}(\mathbf{Y}-\boldsymbol{\mu})$, em que

$$
\boldsymbol{\mu}=\mathbf{X} \boldsymbol{\beta} \quad e \quad \boldsymbol{\Sigma}=\boldsymbol{\Sigma}(\boldsymbol{\alpha})
$$

Os elementos de $\boldsymbol{\alpha}$ serão $\left(\alpha_{1}, \ldots, \alpha_{j}, \ldots, \alpha_{q}\right)^{\top} \operatorname{com} q<m-p$, e a função de verossimilhança para o modelo é dada por

$$
L(\boldsymbol{\beta}, \boldsymbol{\alpha} ; \mathbf{Y})=|\boldsymbol{\Sigma}|^{-1 / 2} c_{2}(1+u / \nu)^{-(\nu+m) / 2},
$$

sendo o logaritmo da função de verossimilhança expresso na forma

$$
l(\boldsymbol{\beta}, \boldsymbol{\alpha} ; \mathbf{Y})=-\frac{1}{2} \log |\boldsymbol{\Sigma}|+\log \left\{c_{2}(1+u / \nu)^{-(\nu+m) / 2}\right\},
$$

em que $c_{2}$ é dado na equação (3.2).

A seguir, ilustramos o caso particular da estimação dos parâmetros do modelo linear t-Student utilizando os resultados obtidos nas seções anteriores para os métodos de estimação na família elíptica.

\section{Estimação de máxima verossimilhança}

Para a estimação da matriz $\boldsymbol{\Sigma}$ usaremos o seguinte resultado da equação (3.7):

$$
-\frac{1}{2} \operatorname{tr}\left\{\boldsymbol{\Sigma}^{-1} \frac{\partial \boldsymbol{\Sigma}}{\partial \alpha_{j}}\right\}-w_{g}(u)(\mathbf{Y}-\mathbf{X} \boldsymbol{\beta})^{\top} \boldsymbol{\Sigma}^{-1} \frac{\partial \boldsymbol{\Sigma}}{\partial \alpha_{j}} \boldsymbol{\Sigma}^{-1}(\mathbf{Y}-\mathbf{X} \boldsymbol{\beta})=\mathbf{0},
$$


para $j=1, \ldots, q$. Da Tabela 3.2 temos que $w_{g}(u)=-\{(\nu+m) / 2 \nu\}(1+u / \nu)^{-1}$. Substituindo na expressão anterior, obtemos

$$
\begin{aligned}
-\frac{1}{2} \operatorname{tr}\left\{\boldsymbol{\Sigma}^{-1} \frac{\partial \boldsymbol{\Sigma}}{\partial \alpha_{j}}\right\}+\frac{\nu+m}{2 \nu}\{ & \left.1+\frac{1}{\nu}(\mathbf{Y}-\mathbf{X} \boldsymbol{\beta})^{\top} \boldsymbol{\Sigma}^{-1}(\mathbf{Y}-\mathbf{X} \boldsymbol{\beta})\right\}^{-1} \\
& \times(\mathbf{Y}-\mathbf{X} \boldsymbol{\beta})^{\top} \boldsymbol{\Sigma}^{-1} \frac{\partial \boldsymbol{\Sigma}}{\partial \alpha_{j}} \boldsymbol{\Sigma}^{-1}(\mathbf{Y}-\mathbf{X} \boldsymbol{\beta})=\mathbf{0}
\end{aligned}
$$

para $j=1, \ldots, q$. Novamente, precisamos definir uma estrutura para a matriz de escala e verificar se o estimador de máxima verossimilhança tem forma explícita. Então, similarmente ao modelo linear normal, precisaremos de algum método de aproximação para encontrar uma estimativa de máxima verossimilhança para a matriz $\boldsymbol{\Sigma}$.

\section{Estimação de máxima verossimilhança restrita}

Da Seção 3.3 temos que a distribuição da transformação $\mathbf{W}^{\top} \mathbf{Y}$ continua sendo elíptica. Neste caso, como consideramos que $\mathbf{Y}$ tem distribuição t-Student multivariada, usando a notação proposta na Tabela 3.1, temos que

$$
\begin{gathered}
\mathbf{W}^{\top} \mathbf{Y} \sim t_{m}\left(\mathbf{W}^{\top} X \boldsymbol{\beta}, \mathbf{W}^{\top} \mathbf{\Sigma} \mathbf{W}, \nu\right) \\
{\left[\begin{array}{c}
\mathbf{W}_{1}^{\top} \mathbf{Y} \\
\mathbf{W}_{2}^{\top} \mathbf{Y}
\end{array}\right]=\left[\begin{array}{c}
\mathbf{V}_{1} \\
\mathbf{V}_{2}
\end{array}\right] \sim t_{m}\left(\left[\begin{array}{c}
\boldsymbol{\beta} \\
\mathbf{0}
\end{array}\right],\left[\begin{array}{ll}
\mathbf{W}_{1}^{\top} \mathbf{\Sigma} \mathbf{W}_{1} & \mathbf{W}_{1}^{\top} \mathbf{\Sigma} \mathbf{W}_{2} \\
\mathbf{W}_{2}^{\top} \mathbf{\Sigma} \mathbf{W}_{1} & \mathbf{W}_{2}^{\top} \mathbf{\Sigma} \mathbf{W}_{2}
\end{array}\right], \nu\right) .}
\end{gathered}
$$

A distribuição marginal de $\mathbf{V}_{2}$ considerando a equação (3.8), temos que no caso da distribuição tStudent multivariada, as marginais continuam tendo a mesma distribuição. Então, podemos denotar

$$
\mathbf{W}_{2}^{\top} \mathbf{Y}=\mathbf{V}_{2} \sim t_{m-p}\left(\mathbf{0}, \mathbf{W}_{2}^{\top} \boldsymbol{\Sigma} \mathbf{W}_{2}, \nu\right)
$$

Sabemos da equação (3.15), que o estimador de $\boldsymbol{\beta}$ não depende da função $g(\cdot)$. Isto é, o estimador tem a mesma estrutura para qualquer distribuição que pertença ao modelo linear elíptico. Da mesma equação, concluímos que o estimador não é afetado pela transformação. Portanto, não precisamos determinar a distribuição condicional de $\mathbf{V}_{1} \mid \mathbf{V}_{2}$. A derivada do logaritmo da função de verossimilhança de $\mathbf{V}_{2}$ com respeito a $\alpha_{j}$ fica dada por

$$
\begin{aligned}
\frac{\partial l\left(\boldsymbol{\alpha} ; \mathbf{V}_{2}\right)}{\partial \alpha_{j}}= & -\frac{1}{2} \operatorname{tr}\left\{\boldsymbol{\Sigma}^{-1} \frac{\partial \boldsymbol{\Sigma}}{\partial \alpha_{j}}\right\}+\frac{1}{2} \operatorname{tr}\left\{\left(\mathbf{X}^{\top} \boldsymbol{\Sigma}^{-1} \mathbf{X}\right)^{-1} \mathbf{X}^{\top} \boldsymbol{\Sigma}^{-1} \frac{\partial \boldsymbol{\Sigma}}{\partial \alpha_{j}} \boldsymbol{\Sigma}^{-1} \mathbf{X}\right\} \\
+ & \frac{\nu+m-p}{2 \nu}\left\{1+\frac{1}{\nu}(\mathbf{Y}-\mathbf{X} \mathbf{b})^{\top} \boldsymbol{\Sigma}^{-1}(\mathbf{Y}-\mathbf{X} \mathbf{b})\right\}^{-1} \\
& \times(\mathbf{Y}-\mathbf{X} \mathbf{b})^{\top} \boldsymbol{\Sigma}^{-1} \frac{\partial \boldsymbol{\Sigma}}{\partial \alpha_{j}} \boldsymbol{\Sigma}^{-1}(\mathbf{Y}-\mathbf{X} \mathbf{b}),
\end{aligned}
$$

para $j=1, \ldots, q$ e lembrando que $\mathbf{b}=\left(\mathbf{X}^{\top} \boldsymbol{\Sigma}^{-1} \mathbf{X}\right)^{-1} \mathbf{X}^{\top} \boldsymbol{\Sigma}^{-1} \mathbf{Y}$

\section{Estimação de máxima verossimilhança perfilada modificada}

O logaritmo da função de verossimilhança perfilada de $\boldsymbol{\alpha}$, considerando que $\hat{\boldsymbol{\beta}}_{\alpha}=\left(\mathbf{X}^{\top} \boldsymbol{\Sigma}^{-1} \mathbf{X}\right)^{-1} \mathbf{X}^{\top} \boldsymbol{\Sigma}^{-1} \mathbf{Y}$, fica dado por

$$
l_{p}(\boldsymbol{\alpha})=-\frac{1}{2} \log |\boldsymbol{\Sigma}|+\log c_{2}-\frac{\nu+m}{2} \log \left\{1+\frac{1}{\nu}\left(\mathbf{Y}-\mathbf{X} \hat{\boldsymbol{\beta}}_{\alpha}\right)^{\top} \boldsymbol{\Sigma}^{-1}\left(\mathbf{Y}-\mathbf{X} \hat{\boldsymbol{\beta}}_{\alpha}\right)\right\} .
$$


Escrevendo o ajuste da função de verossimilhança perfilada modificada de $\boldsymbol{\alpha}$, obtemos

$$
\begin{aligned}
l_{p m}^{*}(\boldsymbol{\alpha}) & =l_{p}(\boldsymbol{\alpha})-\frac{1}{2} \log \left|\mathbf{J}_{\beta \beta}\left(\hat{\boldsymbol{\beta}}_{\alpha}, \boldsymbol{\alpha}\right)\right| \\
& \left.=l_{p}(\boldsymbol{\alpha})-\frac{1}{2} \log \left\{\mid 2 w_{g}\left(\hat{u}_{\alpha}\right) \mathbf{X}^{\top} \boldsymbol{\Sigma}^{-1} \mathbf{X}\right\} \mid\right\} .
\end{aligned}
$$

As funções $w_{g}\left(\hat{u}_{\alpha}\right)$ e $w_{g}^{\prime}\left(\hat{u}_{\alpha}\right)$ para a distribuição t-Student foram especificadas na Tabela 3.2. Considerando a equação $(3.19)$ temos que a função $C_{g}(\boldsymbol{\alpha})$ fica dada por

$$
\begin{aligned}
\frac{2 w_{g}\left(\hat{u}_{\alpha}\right)^{2}-p w_{g}^{\prime}\left(\hat{u}_{\alpha}\right)}{2 w_{g}\left(\hat{u}_{\alpha}\right)} & =\frac{2\left\{-(\nu+m) /(2 \nu)\left(1+\hat{u}_{\alpha} / \nu\right)^{-1}\right\}^{2}-p(\nu+m) /\left(2 \nu^{2}\right)\left(1+\hat{u}_{\alpha} / \nu\right)^{-2}}{-2(\nu+m) /(2 \nu)\left(1+\hat{u}_{\alpha} / \nu\right)^{-1}} \\
& =-\frac{\nu+m-p}{2 \nu}\left(1+\hat{u}_{\alpha} / \nu\right)^{-1} .
\end{aligned}
$$

Assim, a derivada da função de verossimilhança perfilada modificada com respeito a $\alpha_{j}$, para a distribuição t-Student, fica dada por

$$
\begin{aligned}
\frac{\partial}{\partial \alpha_{j}} l_{p m}^{*}(\boldsymbol{\alpha})= & -\frac{1}{2} \operatorname{tr}\left\{\boldsymbol{\Sigma}^{-1} \frac{\partial \boldsymbol{\Sigma}}{\partial \alpha_{j}}\right\}+\frac{1}{2} \operatorname{tr}\left\{\left(\mathbf{X}^{\top} \boldsymbol{\Sigma}^{-1} \mathbf{X}\right)^{-1} \mathbf{X}^{\top} \boldsymbol{\Sigma}^{-1} \frac{\partial \boldsymbol{\Sigma}}{\partial \alpha_{j}} \boldsymbol{\Sigma}^{-1} \mathbf{X}\right\} \\
- & \frac{\nu+m-p}{2 \nu}\left(1+(1 / \nu)\left(\mathbf{Y}-\mathbf{X} \hat{\boldsymbol{\beta}}_{\alpha}\right)^{\top} \boldsymbol{\Sigma}^{-1}\left(\mathbf{Y}-\mathbf{X} \hat{\boldsymbol{\beta}}_{\alpha}\right)\right)^{-1} \\
& \times\left(\mathbf{Y}-\mathbf{X} \hat{\boldsymbol{\beta}}_{\alpha}\right)^{\top} \boldsymbol{\Sigma}^{-1} \frac{\partial \boldsymbol{\Sigma}}{\partial \alpha_{j}} \boldsymbol{\Sigma}^{-1}\left(\mathbf{Y}-\mathbf{X} \hat{\boldsymbol{\beta}}_{\alpha}\right),
\end{aligned}
$$

para $j=1, \ldots, q$.

Utilizando a matriz de informação esperada no lugar da observada, obtemos uma aproximação do logaritmo da função de verossimilhança perfilada modificada. Da expressão (3.22) temos que a matriz de informação esperada com respeito a $\boldsymbol{\beta}$, avaliada em $\boldsymbol{\beta}=\hat{\boldsymbol{\beta}}_{\alpha}$, assume a forma

$$
\mathbf{K}_{\beta \beta}\left(\hat{\boldsymbol{\beta}}_{\alpha}, \boldsymbol{\alpha}\right)=\left.\frac{4}{m} \mathrm{E}\left(u w_{g}^{2}(u)\right)\right|_{\beta=\hat{\beta}_{\alpha}} \mathbf{X}^{\top} \boldsymbol{\Sigma}^{-1} \mathbf{X} .
$$

Sabemos pela Tabela 3.2 que a função $w_{g}(u)$ para a distribuição t-Student é dada por $-\{(\nu+$ $m) /(2 \nu)\}(1+u / \nu)^{-1}$. Então,

$$
\begin{aligned}
\mathbf{K}_{\beta \beta}\left(\hat{\boldsymbol{\beta}}_{\alpha}, \boldsymbol{\alpha}\right) & =\left.\frac{4}{m} \mathrm{E}\left(u\left[-\{(\nu+m) /(2 \nu)\}(1+u / \nu)^{-1}\right]^{2}\right)\right|_{\beta=\hat{\beta}_{\alpha}} \mathbf{X}^{\top} \boldsymbol{\Sigma}^{-1} \mathbf{X} \\
& =\frac{4}{m} \frac{m}{4 \nu^{2}}\left(\frac{\nu+m}{\nu+m+2}\right) \mathbf{X}^{\top} \boldsymbol{\Sigma}^{-1} \mathbf{X} \\
& =\frac{\nu+m}{(\nu+m+2) \nu^{2}} \mathbf{X}^{\top} \boldsymbol{\Sigma}^{-1} \mathbf{X} .
\end{aligned}
$$

O cálculo da esperança de um vetor aleatório com distribuição t-Student multivariada foi desenvolvido por Lange et al. (1989). Observamos que o valor de $\left.\mathrm{E}\left(u w_{g}^{2}(u)\right)\right|_{\beta=\hat{\beta}_{\alpha}}$ é constante com respeito a $\boldsymbol{\Sigma}$.

Escrevendo o ajuste do logaritmo da função de verossimilhança perfilada modificada aproximada de $\boldsymbol{\alpha}$ e utilizando a matriz de informação esperada no lugar da observada, obtemos

$$
\begin{aligned}
l_{p m a}^{*}(\boldsymbol{\alpha}) & =l_{p}(\boldsymbol{\alpha})-\frac{1}{2} \log \left|\mathbf{K}_{\beta \beta}\left(\hat{\boldsymbol{\beta}}_{\alpha}, \boldsymbol{\alpha}\right)\right| \\
& =l_{p}(\boldsymbol{\alpha})-\frac{1}{2} \log \left|\frac{\nu+m}{(\nu+m+2) \nu^{2}} \mathbf{X}^{\top} \boldsymbol{\Sigma}^{-1} \mathbf{X}\right|
\end{aligned}
$$

Vemos que o fator que pondera a matriz $\mathbf{X}^{\top} \boldsymbol{\Sigma}^{-1} \mathbf{X}$ não depende de $\boldsymbol{\Sigma}$. Portanto, a derivada da 
expressão anterior com respeito a $\alpha_{j}$ fica dada por

$$
\begin{aligned}
\frac{\partial}{\partial \alpha_{j}} l_{p m a}^{*}(\boldsymbol{\alpha})= & -\frac{1}{2} \operatorname{tr}\left\{\boldsymbol{\Sigma}^{-1} \frac{\partial \boldsymbol{\Sigma}}{\partial \alpha_{j}}\right\}+\frac{1}{2} \operatorname{tr}\left\{\left(\mathbf{X}^{\top} \boldsymbol{\Sigma}^{-1} \mathbf{X}\right)^{-1} \mathbf{X}^{\top} \boldsymbol{\Sigma}^{-1} \frac{\partial \boldsymbol{\Sigma}}{\partial \alpha_{j}} \boldsymbol{\Sigma}^{-1} \mathbf{X}\right\} \\
& +\frac{\nu+m}{2 \nu}\left\{1+\frac{1}{\nu}\left(\mathbf{Y}-\mathbf{X} \hat{\boldsymbol{\beta}}_{\alpha}\right)^{\top} \boldsymbol{\Sigma}^{-1}\left(\mathbf{Y}-\mathbf{X} \hat{\boldsymbol{\beta}}_{\alpha}\right)\right\}^{-1} \\
& \times\left(\mathbf{Y}-\mathbf{X} \hat{\boldsymbol{\beta}}_{\alpha}\right)^{\top} \boldsymbol{\Sigma}^{-1} \frac{\partial \boldsymbol{\Sigma}}{\partial \alpha_{j}} \boldsymbol{\Sigma}^{-1}\left(\mathbf{Y}-\mathbf{X} \hat{\boldsymbol{\beta}}_{\alpha}\right),
\end{aligned}
$$

$\operatorname{para} j=1, \ldots, q$.

Para a distribuição t-Student obtemos as mesmas equações de estimação no método restrito (3.29) e perfilada modificada (3.30), mas diferem da equação de estimação perfilada modificada aproximada (3.32) no fator que pondera o último termo.

\section{Estruturas para a matriz de escala}

Consideremos o caso particular para a matriz $\boldsymbol{\Sigma}=\sigma^{2} \mathbf{I}_{m}$. Assim, comparamos as expressões obtidas entre os métodos. Para tanto, utilizaremos os resultados obtidos da Seção 3.5. Então, não precisamos falar novamente do estimador de $\boldsymbol{\beta}$ pois este não depende de $g(\cdot)$ nem de $\sigma^{2}$.

Para a determinação do estimador de máxima verossimilhança de $\sigma^{2}$ consideremos a equação (3.24) e que $w_{g}(u)=-\{(\nu+m) / 2\}(\nu+u)^{-1}$. Então, obtemos a equação de estimação

$$
-\sigma^{2} \nu-(\mathbf{Y}-\mathbf{X} \boldsymbol{\beta})^{\top}(\mathbf{Y}-\mathbf{X} \boldsymbol{\beta})+\frac{\nu+m}{m}(\mathbf{Y}-\mathbf{X} \boldsymbol{\beta})^{\top}(\mathbf{Y}-\mathbf{X} \boldsymbol{\beta})=0 .
$$

Podemos concluir que o estimador de máxima verossimilhança para $\sigma^{2}$ fica dado por

$$
\hat{\sigma}_{M V}^{2}=\frac{1}{m}(\mathbf{Y}-\mathbf{X} \hat{\boldsymbol{\beta}})^{\top}(\mathbf{Y}-\mathbf{X} \hat{\boldsymbol{\beta}}) .
$$

O estimador resultante é o mesmo do caso normal, mas aqui $\sigma^{2}$ não representa a variância de $\mathbf{Y}$. Para o estimador de máxima verossimilhança restrita temos desde (3.24) que,

$$
-\sigma^{2}-\frac{2 w_{2}\left(u_{2}\right)}{m-p}(\mathbf{Y}-\mathbf{X b})^{\top}(\mathbf{Y}-\mathbf{X b})=0 .
$$

Da Seção 3.5, temos que $u_{2}=(\mathbf{Y}-\mathbf{X b})^{\top} \boldsymbol{\Sigma}^{-1}(\mathbf{Y}-\mathbf{X b})$ e para $\boldsymbol{\Sigma}=\sigma^{2} \mathbf{I}_{m}$,

$$
u_{2}=\frac{1}{\sigma^{2}}(\mathbf{Y}-\mathbf{X} \mathbf{b})^{\top}(\mathbf{Y}-\mathbf{X} \mathbf{b}) .
$$

Então, temos que a equação de estimação resultante fica dada por

$$
-\sigma^{2}\left\{1+\frac{1}{\sigma^{2} \nu}(\mathbf{Y}-\mathbf{X b})^{\top}(\mathbf{Y}-\mathbf{X} \mathbf{b})\right\}+\frac{\nu+m-p}{m-p}(\mathbf{Y}-\mathbf{X b})^{\top}(\mathbf{Y}-\mathbf{X} \mathbf{b})=0 .
$$

Dado que a equação de estimação restrita (3.29) é a mesma que a equação de verossimilhança perfilada modificada (3.30), o estimador resultante fica dado por

$$
\hat{\sigma}_{M V R}^{2}=\hat{\sigma}_{M V P M}^{2}=\frac{1}{m-p}(\mathbf{Y}-\mathbf{X} \hat{\boldsymbol{\beta}})^{\top}(\mathbf{Y}-\mathbf{X} \hat{\boldsymbol{\beta}})
$$

Por último, calcularemos o estimador de máxima verossimilhança perfilada modificada aproxi- 
mada para $\sigma^{2}$. Desde a equação (3.22) temos que,

$$
-\sigma^{2}\left\{+\frac{1}{\sigma^{2} \nu}(\mathbf{Y}-\mathbf{X} \boldsymbol{\beta})^{\top}(\mathbf{Y}-\mathbf{X} \boldsymbol{\beta})\right\}+\frac{\nu+m}{m-p}(\mathbf{Y}-\mathbf{X} \boldsymbol{\beta})^{\top}(\mathbf{Y}-\mathbf{X} \boldsymbol{\beta})=0 .
$$

Nesse caso, $\left.\mathrm{E}\left(u w_{g}^{2}(u)\right)\right|_{\beta=\hat{\beta}_{\alpha}}$ é constante com respeito a $\sigma^{2}$. Assim, temos que o estimador de máxima verossimilhança perfilada modificada fica dado por,

$$
\hat{\sigma}_{M V P M A}^{2}=\frac{\nu+p}{\nu(m-p)}(\mathbf{Y}-\mathbf{X} \hat{\boldsymbol{\beta}})^{\top}(\mathbf{Y}-\mathbf{X} \hat{\boldsymbol{\beta}}) .
$$

Podemos concluir que para a estrutura da matriz $\boldsymbol{\Sigma}=\sigma^{2} \mathbf{I}_{m}$ considerando a distribuição tStudent o estimador de máxima verossimilhança perfilada modificada de $\sigma^{2}$ e perfilada modificada aproximada diferem no fator que pondera a forma quadrática $(\mathbf{Y}-\mathbf{X} \hat{\boldsymbol{\beta}})^{\top}(\mathbf{Y}-\mathbf{X} \hat{\boldsymbol{\beta}})$. No caso deste último, o estimador fica dependendo dos graus de liberdade. Por último, vemos que o estimador de máxima verossimilhança e o estimador de máxima verossimilhança restrita para $\sigma^{2}$ têm a mesma forma do caso normal. Mas devemos observar que a variância de $\mathbf{Y}$ com distribuição t-Student é dada por $\nu /(\nu-2) \sigma^{2}$.

\subsubsection{Modelo linear exponencial potência}

Nesta seção usaremos a definição de distribuição exponencial potência multivariada proposta por Gómez et al. (1998) e também as propriedades apresentadas pelos autores. Vamos considerar uma distribuição exponencial potência multivariada com parâmetros $\boldsymbol{\mu}, \boldsymbol{\Sigma}$ e $\lambda$, em que $\lambda$ é um parâmetro escalar de forma, associado à curtose da distribuição e assumiremos que é conhecido. Então, estimaremos os parâmetros associados ao vetor $\boldsymbol{\mu}$ e à matriz $\boldsymbol{\Sigma}$, lembrando que os parâmetros de interesse são o vetor $\boldsymbol{\beta}(p \times 1)$, em que $\boldsymbol{\mu}=\mathbf{X} \boldsymbol{\beta}$, e o vetor $\boldsymbol{\alpha}$ cujos componentes definem a matriz $\Sigma$.

Como mostramos na Tabela 3.1, a distribuição exponencial potência multivariada também é elíptica e a função $g(u)$ será igual a $c_{3} \exp \left(-u^{\lambda} / 2\right)$, com $c_{3}$ dado em (3.2).

\section{Estrutura do modelo}

Seja Y um vetor aleatório com distribuição exponencial potência e parâmetros $\boldsymbol{\mu}_{(m \times 1)}$ e $\Sigma_{(m \times m)}$, que denotaremos como

$$
\mathbf{Y} \sim E P_{m}(\boldsymbol{\mu}, \boldsymbol{\Sigma}, \lambda)
$$

O $\lambda$ é um parâmetro de forma associado à curtose e vamos a considerar que $\lambda \in(0,1]$. A curtose é uma função decrescente com respeito a $\lambda$. Assim, para $\lambda=1$ temos a distribuição normal e para $\lambda=1 / 2$ temos a distribuição exponencial dupla que tem cuadas mais leve que as caudas da distribuição normal. Considerando a variável $u$ sendo a forma quadrática $(\mathbf{Y}-\boldsymbol{\mu})^{\top} \boldsymbol{\Sigma}^{-1}(\mathbf{Y}-\boldsymbol{\mu})$, em que

$$
\boldsymbol{\mu}=\mathbf{X} \boldsymbol{\beta} \quad e \quad \boldsymbol{\Sigma}=\boldsymbol{\Sigma}(\boldsymbol{\alpha}) .
$$

Os elementos de $\boldsymbol{\alpha}$ serão $\left(\alpha_{1}, \ldots, \alpha_{j}, \ldots, \alpha_{q}\right)^{\top} \operatorname{com} q<m-p$ e a função de verossimilhança para o modelo é dada por

$$
L(\boldsymbol{\beta}, \boldsymbol{\alpha} ; \mathbf{Y})=|\boldsymbol{\Sigma}|^{-1 / 2} c_{3} \exp \left(-\frac{u^{\lambda}}{2}\right)
$$

sendo o logaritmo da função de verossimilhança expresso na forma

$$
l(\boldsymbol{\beta}, \boldsymbol{\alpha} ; \mathbf{Y})=-\frac{1}{2} \log |\boldsymbol{\Sigma}|+\log \left\{c_{3}\right\}-\frac{u^{\lambda}}{2} .
$$

A seguir, ilustramos o caso particular da estimação dos parâmetros da distribuição exponencial potência utilizando os resultados obtidos para o modelo linear elíptico, para os métodos de estimação apresentados nas Seções 3.2, 3.3 e 3.4. 


\section{Estimação de máxima verossimilhança}

Considerando a equação (3.7) do caso elíptico para a estimação de $\alpha_{j}$, com $j=1, \ldots, q$ e da Tabela 3.2 temos que $w_{g}(u)=-1 / 2 \lambda u^{\lambda-1}$. Assim, neste caso a equação de estimação fica dada por

$$
\begin{aligned}
-\frac{1}{2} \operatorname{tr}\left\{\boldsymbol{\Sigma}^{-1} \frac{\partial \boldsymbol{\Sigma}}{\partial \alpha_{j}}\right\}- & \frac{\lambda}{2}\left\{(\mathbf{Y}-\mathbf{X} \boldsymbol{\beta})^{\top} \boldsymbol{\Sigma}^{-1}(\mathbf{Y}-\mathbf{X} \boldsymbol{\beta})\right\}^{\lambda-1} \\
& \times(\mathbf{Y}-\mathbf{X} \boldsymbol{\beta})^{\top} \boldsymbol{\Sigma}^{-1} \frac{\partial \boldsymbol{\Sigma}}{\partial \alpha_{j}} \boldsymbol{\Sigma}^{-1}(\mathbf{Y}-\mathbf{X} \boldsymbol{\beta})=0 .
\end{aligned}
$$

Obtemos uma expressão geral para um elemento do vetor $\alpha_{j}$, para $j=1, \ldots, q$.

\section{Estimação de máxima verossimilhança restrita}

Desde a Seção 3.3 temos que a distribuição da transformação $\mathbf{W}^{\top} \mathbf{Y}$ continua sendo elíptica. Então, consideramos que $\mathbf{Y}$ tem distribuição exponencial potência multivariada, como $\mathbf{W}$ é uma matriz quadrada $(m \times m)$ uma transformação dessa matriz tem a mesma distribuição. Assim, usando a notação proposta na Tabela 3.1, temos que,

$$
\begin{gathered}
\mathbf{V}=\mathbf{W}^{\top} \mathbf{Y} \sim E P_{m}\left(\mathbf{W}^{\top} X \boldsymbol{\beta}, \mathbf{W}^{\top} \mathbf{\Sigma} \mathbf{W}, \lambda\right) \\
{\left[\begin{array}{c}
\mathbf{W}_{1}^{\top} \mathbf{Y} \\
\mathbf{W}_{2}^{\top} \mathbf{Y}
\end{array}\right]=\left[\begin{array}{c}
\mathbf{V}_{1} \\
\mathbf{V}_{2}
\end{array}\right] \sim E P_{m}\left(\left[\begin{array}{c}
\boldsymbol{\beta} \\
\mathbf{0}
\end{array}\right],\left[\begin{array}{ll}
\mathbf{W}_{1}^{\top} \mathbf{\Sigma} \mathbf{W}_{1} & \mathbf{W}_{1}^{\top} \mathbf{\Sigma} \mathbf{W}_{2} \\
\mathbf{W}_{2}^{\top} \mathbf{\Sigma} \mathbf{W}_{1} & \mathbf{W}_{2}^{\top} \mathbf{\Sigma} \mathbf{W}_{2}
\end{array}\right], \lambda\right) .}
\end{gathered}
$$

A distribuição marginal de $\mathbf{V}_{2}$ e a distribuição condicional de $\mathbf{V}_{1} \mid \mathbf{V}_{2}$, para o modelo elíptico foram dadas nas equações (3.8) e (3.9), respectivamente, em que $g_{2}(\cdot)$ é a função geradora de densidade da transformação $\mathbf{V}_{2}$ e é denotada por

$$
\mathbf{W}_{2}^{\top} \mathbf{Y}=\mathbf{V}_{2} \sim E l_{m-p}\left(\mathbf{0}, \mathbf{W}_{2}^{\top} \boldsymbol{\Sigma} \mathbf{W}_{2}, g_{2}\right)
$$

Para a distribuição exponencial potência multivariada, temos que suas marginais não tem distribuição exponencial potência, e devem ser obtidas desde o cálculo da seguinte integral:

$$
g_{2}\left(u_{2}\right)=u_{2}^{\frac{m-p}{2}} \int_{0}^{1} w^{\frac{p-m}{2}-1} \exp \left\{-\frac{1}{2}\left(\frac{u_{2}}{w}\right)^{\lambda}\right\} d w .
$$

Como vimos no modelo geral das distribuições elípticas o estimador de $\boldsymbol{\beta}$ não depende da função $g(\cdot)$, portanto não precisamos determinar a distribuição condicional, $\mathbf{V}_{1} \mid \mathbf{V}_{2}$.

As equações de estimação de $\alpha_{j}, j=1, \ldots, q$, são obtidas das derivadas parciais da densidade marginal de $\mathbf{V}_{2}$ e ficam dadas por

$$
\begin{aligned}
\frac{\partial l\left(\boldsymbol{\alpha} ; \mathbf{V}_{2}\right)}{\partial \alpha_{j}}= & \frac{1}{2} \operatorname{tr}\left\{\left(\mathbf{X}^{\top} \boldsymbol{\Sigma}^{-1} \mathbf{X}\right)^{-1} \mathbf{X}^{\top} \boldsymbol{\Sigma}^{-1} \frac{\partial \boldsymbol{\Sigma}}{\partial \alpha_{j}} \boldsymbol{\Sigma}^{-1} \mathbf{X}\right\}-\frac{1}{2} \operatorname{tr}\left\{\boldsymbol{\Sigma}^{-1} \frac{\partial \boldsymbol{\Sigma}}{\partial \alpha_{j}}\right\} \\
& -w_{2}\left(u_{2}\right)(\mathbf{Y}-\mathbf{X} \mathbf{b})^{\top} \boldsymbol{\Sigma}^{-1} \frac{\partial \boldsymbol{\Sigma}}{\partial \alpha_{j}} \boldsymbol{\Sigma}^{-1}(\mathbf{Y}-\mathbf{X b})
\end{aligned}
$$

em que $w_{2}\left(u_{2}\right)$ fica dada por

$$
\begin{aligned}
& w_{2}\left(u_{2}\right)=\frac{m-p}{2} d \log u_{2}+d \log \int_{0}^{1} w^{\frac{p-m}{2}-1} \exp \left\{-\frac{1}{2}\left(\frac{u_{2}}{w}\right)^{\lambda}\right\} d w \\
& =\frac{m-p}{2 u_{2}}+\frac{d \int_{0}^{1} w^{\frac{p-m}{2}-1} \exp \left\{-\frac{1}{2}\left(\frac{u_{2}}{w}\right)^{\lambda}\right\} d w}{\int_{0}^{1} w^{\frac{p-m}{2}-1} \exp \left\{-\frac{1}{2}\left(\frac{u_{2}}{w}\right)^{\lambda}\right\} d w} .
\end{aligned}
$$


Assim, para obter um estimador restrito para $\alpha_{j}$ precisamos resolver a integral da equação (3.33). Outra forma é considerar o Teorema 1 descrito no artigo Gómez-Sánchez-Manzano et al. (2006), isto é, que a distribuição de $\mathbf{W}^{\top} \mathbf{Y} \operatorname{com} \lambda$ em $(0,1]$ é uma distribuição de mistura de escala normal, denotada por $\mathbf{V}=\mathbf{W}^{\top} \mathbf{Y} \sim S M N_{m}\left(\mathbf{W}^{\top} X \boldsymbol{\beta}, \mathbf{W}^{\top} \boldsymbol{\Sigma} \mathbf{W}, F_{\lambda}\right)$, em que $F_{\lambda}$ é a função de distribuição e $f_{\lambda}$ é a função de densidade especificada na equação (2.1) desse artigo. Uma consequência do Teorema 1 é que as distribuições marginais de $\mathbf{V}$ continuam sendo mistura de escala normal. Neste caso, temos que $\mathbf{V}_{2} \sim S M N_{m-p}\left(\mathbf{0}, \mathbf{W}_{2}^{\top} \boldsymbol{\Sigma} \mathbf{W}_{2}, F_{\lambda}\right)$, em que $F_{\lambda}$ é a mesma função de distribuição de $\mathbf{V}$ em que $f_{n}$.

\section{Estimação de máxima verossimilhança perfilada modificada}

Considerando que o estimador de máxima verossimilhança de $\boldsymbol{\beta}$ para $\boldsymbol{\alpha}$ fixado é dado por $\hat{\boldsymbol{\beta}}_{\alpha}=\left(\mathbf{X}^{\top} \boldsymbol{\Sigma}^{-1} \mathbf{X}\right)^{-1} \mathbf{X}^{\top} \boldsymbol{\Sigma}^{-1} Y$, temos que a função de verossimilhança perfilada de $\boldsymbol{\alpha}$ fica dada por

$$
l_{p}(\boldsymbol{\alpha})=-\frac{1}{2} \log |\boldsymbol{\Sigma}|-\frac{1}{2}\left\{\left(\mathbf{Y}-\mathbf{X} \hat{\boldsymbol{\beta}}_{\alpha}\right)^{\top} \boldsymbol{\Sigma}^{-1}\left(\mathbf{Y}-\mathbf{X} \hat{\boldsymbol{\beta}}_{\alpha}\right)\right\}^{\lambda} .
$$

O ajuste da função de verossimilhança perfilada modificada de $\boldsymbol{\alpha}$ fica dado por

$$
l_{p m}^{*}(\boldsymbol{\alpha})=l_{p}(\boldsymbol{\alpha})-\frac{1}{2} \log \left|2 w_{g}\left(\hat{u}_{\alpha}\right) \mathbf{X}^{\top} \boldsymbol{\Sigma}^{-1} \mathbf{X}\right|,
$$

em que as funções $w_{g}\left(\hat{u}_{\alpha}\right)$ e $w_{g}^{\prime}\left(\hat{u}_{\alpha}\right)$ para a distribuição exponencial potência foram especificadas na Tabela 3.2. A função $C_{g}(\boldsymbol{\alpha})$ que foi determinada na equação (3.19) para esta distribuição fica dada por

$$
\begin{aligned}
\frac{2 w_{g}\left(\hat{u}_{\alpha}\right)^{2}-p w_{g}^{\prime}\left(\hat{u}_{\alpha}\right)}{2 w_{g}\left(\hat{u}_{\alpha}\right)} & =\frac{2\left\{-(1 / 2) \lambda \hat{u}_{\alpha}^{\lambda-1}\right\}^{2}+p(1 / 2) \lambda(1-\lambda) \hat{u}_{\alpha}^{\lambda-2}}{-2(1 / 2) \lambda \hat{u}_{\alpha}^{\lambda-1}} \\
& =\frac{1}{2}\left(\lambda \hat{u}_{\alpha}^{\lambda-1}-p(\lambda-1) \hat{u}_{\alpha}^{-1}\right) .
\end{aligned}
$$

Considerando derivar a função de verossimilhança perfilada modificada para um modelo elíptico (equação (3.19)) temos para $\alpha_{j}$ que

$$
\begin{aligned}
\frac{\partial}{\partial \alpha_{j}} l_{p m}^{*}(\alpha) & =-\frac{1}{2} \operatorname{tr}\left\{\boldsymbol{\Sigma}^{-1} \frac{\partial \boldsymbol{\Sigma}}{\partial \alpha_{j}}\right\}+\frac{1}{2} \operatorname{tr}\left\{\left(\mathbf{X}^{\top} \boldsymbol{\Sigma}^{-1} \mathbf{X}\right)^{-1} \mathbf{X}^{\top} \boldsymbol{\Sigma}^{-1} \frac{\partial \boldsymbol{\Sigma}}{\partial \alpha_{j}} \boldsymbol{\Sigma}^{-1} \mathbf{X}\right\} \\
& +\frac{1}{2}\left(\lambda \hat{u}_{\alpha}^{\lambda-1}-p(\lambda-1) \hat{u}_{\alpha}^{-1}\right)\left(\mathbf{Y}-\mathbf{X} \hat{\boldsymbol{\beta}}_{\alpha}\right)^{\top} \boldsymbol{\Sigma}^{-1} \frac{\partial \boldsymbol{\Sigma}}{\partial \alpha_{j}} \boldsymbol{\Sigma}^{-1}\left(\mathbf{Y}-\mathbf{X} \hat{\boldsymbol{\beta}}_{\alpha}\right)
\end{aligned}
$$

lembrando que $\hat{u}_{\alpha}=\left(\mathbf{Y}-\mathbf{X} \hat{\boldsymbol{\beta}}_{\alpha}\right)^{\top} \boldsymbol{\Sigma}^{-1}\left(\mathbf{Y}-\mathbf{X} \hat{\boldsymbol{\beta}}_{\alpha}\right), j=1, \ldots, q$.

Por último, para determinar a aproximação da função de verossimilhança perfilada modificada aproximada devemos calcular a matriz de informação esperada de $\boldsymbol{\beta}$ avaliada em $\boldsymbol{\beta}=\hat{\boldsymbol{\beta}}_{\alpha}$. Então, da equação (3.20), temos que

$$
\mathbf{K}_{\beta \beta}\left(\hat{\boldsymbol{\beta}}_{\alpha}, \boldsymbol{\alpha}\right)=\left.\frac{4}{m} \mathrm{E}\left(u w_{g}^{2}(u)\right)\right|_{\beta=\hat{\beta}_{\alpha}} \mathbf{X}^{\top} \boldsymbol{\Sigma}^{-1} \mathbf{X} .
$$

Sabemos desde a Tabela 3.2 que $w_{g}(u)=-1 / 2 \lambda u^{\lambda-1}$, então

$$
\begin{aligned}
\mathbf{K}_{\beta \beta}\left(\hat{\boldsymbol{\beta}}_{\alpha}, \boldsymbol{\alpha}\right) & =\left.\frac{4}{m} \mathrm{E}\left(u\left(-1 / 2 \lambda u^{\lambda-1}\right)^{2}\right)\right|_{\beta=\hat{\beta}_{\alpha}} \mathbf{X}^{\top} \boldsymbol{\Sigma}^{-1} \mathbf{X} \\
& =\left.\frac{\lambda^{2}}{m} \mathrm{E}\left(u^{2 \lambda-1}\right)\right|_{\beta=\hat{\beta}_{\alpha}} \mathbf{X}^{\top} \boldsymbol{\Sigma}^{-1} \mathbf{X} .
\end{aligned}
$$

Para calcular a esperança consideraremos a representação estocástica de um vetor aleatório $\mathbf{Y}$ com distribuição elíptica, apresentada no Apêndice C. Então, temos que $\mathbf{Y}-\boldsymbol{\mu} \stackrel{d}{=} r \mathbf{A}^{\top} \mathbf{s}$, com $\mathbf{A}^{\top} \mathbf{A}=\boldsymbol{\Sigma}$. 
Então, obtemos,

$$
\begin{aligned}
\mathrm{E}\left(u^{2 \lambda-1}\right) & =\mathrm{E}\left[\left\{(\mathbf{Y}-\boldsymbol{\mu})^{\top} \boldsymbol{\Sigma}^{-1}(\mathbf{Y}-\boldsymbol{\mu})\right\}^{2 \lambda-1}\right] \\
& =\mathrm{E}\left[\left\{\left(r \mathbf{A}^{\top} \mathbf{s}\right)^{\top} \boldsymbol{\Sigma}^{-1}\left(r \mathbf{A}^{\top} \mathbf{s}\right)\right\}^{2 \lambda-1}\right] \\
& =\mathrm{E}\left\{\left(r^{2} \mathbf{s}^{\top} \mathbf{s}\right)^{2 \lambda-1}\right\} .
\end{aligned}
$$

Nesta última igualdade vemos que $\mathrm{E}\left(u^{2 \lambda-1}\right)$ não depende da matriz $\boldsymbol{\Sigma}$. Assim, não precisamos obter uma expressão para esta esperança. Este resultado simplificará o cálculo do estimador de $\boldsymbol{\Sigma}$. Para a função de verossimilhança perfilada modificada de $\boldsymbol{\alpha}$, utilizaremos a matriz de informação esperada no lugar da observada, obtendo

$$
\begin{aligned}
l_{p m a}^{*}(\boldsymbol{\alpha}) & =l_{p}(\boldsymbol{\alpha})-\frac{1}{2} \log \left|\mathbf{X}^{\top} \boldsymbol{\Sigma}^{-1} \mathbf{X}\right|-\frac{p}{2} \log \left|\frac{4}{2 \pi m} \mathrm{E}\left(u w_{g}^{2}(u)\right)\right|_{\beta=\hat{\beta}_{\alpha}} \mid, \\
& =l_{p}(\boldsymbol{\alpha})-\frac{1}{2} \log \left|\mathbf{X}^{\top} \boldsymbol{\Sigma}^{-1} \mathbf{X}\right|-\frac{p}{2} \log \mid \text { cte } \mid
\end{aligned}
$$

Portanto, a equação de estimação com respeito a $\alpha_{j}$ fica dada por

$$
\begin{aligned}
\frac{\partial}{\partial \alpha_{j}} l_{p m a}^{*}(\boldsymbol{\alpha})= & -\frac{1}{2} \operatorname{tr}\left\{\boldsymbol{\Sigma}^{-1} \frac{\partial \boldsymbol{\Sigma}}{\partial \alpha_{j}}\right\}+\frac{\lambda}{2}\left\{\left(\mathbf{Y}-\mathbf{X} \hat{\boldsymbol{\beta}}_{\alpha}\right)^{\top} \boldsymbol{\Sigma}^{-1}\left(\mathbf{Y}-\mathbf{X} \hat{\boldsymbol{\beta}}_{\alpha}\right)\right\}^{\lambda-1} \\
& \times\left(\mathbf{Y}-\mathbf{X} \hat{\boldsymbol{\beta}}_{\alpha}\right)^{\top} \boldsymbol{\Sigma}^{-1} \frac{\partial \boldsymbol{\Sigma}}{\partial \alpha_{j}} \boldsymbol{\Sigma}^{-1}\left(\mathbf{Y}-\mathbf{X} \hat{\boldsymbol{\beta}}_{\alpha}\right) \\
+ & \frac{1}{2} \operatorname{tr}\left\{\left(\mathbf{X}^{\top} \boldsymbol{\Sigma}^{-1} \mathbf{X}\right)^{-1} \mathbf{X}^{\top} \boldsymbol{\Sigma}^{-1} \frac{\partial \boldsymbol{\Sigma}}{\partial \alpha_{j}} \boldsymbol{\Sigma}^{-1} \mathbf{X}\right\}
\end{aligned}
$$

para $j=1, \ldots, q$. Podemos ver que utilizando a matriz de informação esperada no lugar da matriz de informação observada, simplifica bastante a função de estimação de $\boldsymbol{\Sigma}$.

\subsection{Comentários}

Vimos que os métodos de estimação apresentados podem ser estendidos para o modelo linear elíptico. O estimador de $\boldsymbol{\beta}$, neste tipo de modelo, não depende da função geradora de densidade elíptica $g(\cdot)$. Portanto, similarmente ao caso normal, a estrutura dos estimadores de $\boldsymbol{\beta}$ é a mesma para todos os métodos e para qualquer distribuição elíptica. Contudo, os estimadores de $\boldsymbol{\beta}$ resultantes não serão os mesmos para os três métodos, pois dependem dos estimadores de $\boldsymbol{\Sigma}$. Os estimadores de $\boldsymbol{\Sigma}$ não têm forma fechada para nenhum dos métodos. Se comparamos as equações de estimação vemos que contêm termos diferentes.

Na seção anterior, apresentamos dois casos particulares de distribuições elípticas. No caso da distribuição t-Student multivariada, em que suas marginais continuam tendo distribuição t-Student, vimos que para o caso mais simples de estrutura de escala os estimadores de $\sigma^{2}$ são diferentes para os três métodos. Quando consideramos uma distribuição exponencial potência multivariada, a expressão da função de densidade marginal da transformação $\mathbf{W}^{\top} \mathbf{Y}$ depende de uma integral que deixa mais complexa a obtenção do estimador da matriz de escala para o método de máxima verossimilhança restrita.

Uma relação entre os estimadores de máxima verossimilhança restrita num modelo espacial elíptico foi obtida por Vallejos e Osorio (2015). Os autores utilizam os resultados obtidos por Anderson et al. (1986), que apresentam uma relação entre os estimadores da máxima verossimi- 
lhança sob normalidade e os estimadores de máxima verossimilhança em distribuições elípticas com função geradora $g(\cdot)$. Analisaremos abaixo esta relação para os diferentes métodos de estimação. Considerando $\mathbf{Y} \sim E l_{m}(\boldsymbol{\mu} ; \boldsymbol{\Sigma}, g)$, denotamos $\tilde{\boldsymbol{\mu}}$ e $\tilde{\boldsymbol{\Sigma}}$ os estimadores de máxima verossimilhança sob normalidade. Então, os estimadores de máxima verossimilhança sob modelo elíptico ficam dados por $\hat{\boldsymbol{\mu}}=\tilde{\boldsymbol{\mu}}$ e $\hat{\boldsymbol{\Sigma}}=\frac{m}{y_{g}} \tilde{\boldsymbol{\Sigma}}$, em que $n$ é a dimensão do vetor $\mathbf{Y}$ e $y_{g}=\max \left\{y^{m / 2} g(y)\right\}$. No caso do estimador de $\boldsymbol{\beta}$, como vimos na equação (3.15), esta relação existe para todas as distribuições elípticas que têm função de densidade gerada por $g(\cdot)$ e que existe ao menos sua primeira derivada. Para a estrutura de variância-covariância, como não temos expressões exatas, não podemos obter esta relação direta para o estimador de máxima verossimilhança. Mas a importância dessa relação de estimadores facilitará bastante os cálculos. Isto é, se temos o estimador de máxima verossimilhança para o caso normal e podemos determinar $y_{g}$ temos o estimador de máxima verossimilhança da estrutura da matriz de escala para uma distribuição elíptica.

Na aplicação para o estimador de máxima verossimilhança perfilada modificada, não podemos considerar a relação dos estimadores, pois as funções de verossimilhança resultantes, como seu nome indica, são uma modificação da função de verossimilhança do caso normal e do caso elíptico. Em outras palavras, não estamos relacionando estimadores de máxima verossimilhança que provêm diretamente de uma distribuição normal nem de uma distribuição elíptica. Portanto, a relação não é direta.

No caso da estimação restrita para a estrutura de $\boldsymbol{\Sigma}$, temos que as funções de densidade marginais da transformação continuam tendo distribuição normal e elíptica, respectivamente. Assim, podemos estabelecer a relação entre os estimadores da matriz $\boldsymbol{\Sigma}$, mas levando em conta que, neste caso, a função geradora de densidade é $g_{2}(\cdot)$ correspondente à transformação $\mathbf{W}_{2}^{\top} \mathbf{Y}$ com dimensão $m-p$ e $y_{g}=\max \left\{y^{(m-p) / 2} g(y)\right\}$.

Para comparar as estimativas pelos distintos métodos de estimação, no Capítulo 6 apresentamos simulações de dois modelos lineares dependentes t-Student. Considerando uma aproximação dos errores padrão das estimativas mediante um procedimento bootstrap não paramétrico. Nesse contexto, McCullagh e Tibshirani (1990) propõem, a partir do logaritmo da função de verossimilhança perfilada, um ajuste nas funções escore para que tenha média zero e variância dada por o negativo da esperança da derivada do ajuste das funções escore. Nos modelos lineares normais as estimativas resultantes coincidem com as obtidas pelo ajuste de Cox e Reid (1987) e portanto com os estimadores REML. Mas McCullagh e Tibshirani (1990) apresentam exemplo em que os métodos não coincidem. Smyth e Verbyla (1999) discutem a relação entre o método de McCullagh e Tibshirani (1990) e Cox e Reid (1987) para modelos lineares generalizados duplos.

Por último, consideramos como pesquisa futura a aplicação do ajuste das funções escore da verossimilhança perfilada proposto por McCullagh e Tibshirani (1990) nos modelos lineares elípticos e comparação das estimativas obtidas com o ajuste de Cox e Reid (1987). 


\section{Capítulo 4}

\section{Métodos de estimação no modelo misto linear elíptico}

O modelo misto linear (MML) é bastante flexível pois permite modelar diversos tipos de fenômenos aleatórios, tendo uma grande popularidade em diversas áreas de pesquisa. A estrutura dos modelos lineares mistos contém efeitos fixos e aleatórios, permitindo estudar distintas estruturas de variabilidade. Assim, este tipo de modelo tem sido frequentemente utilizado na análise de medidas repetidas, dados agrupados e dados longitudinais, entre outros. Os modelos mistos lineares têm como casos particulares o modelo linear clássico e o modelo de componentes de variância. Devido à sua grande aplicabilidade tem havido um amplo desenvolvimento de métodos de estimação, inferência, técnicas de diagnóstico e ferramentas computacionais (ver, por exemplo, Demidenko (2013), Pinheiro e Bates (2000), e Verbeke e Molenberghs (2000)). Esta variabilidade tem levado a algumas extensões, como o modelo misto linear generalizado, o modelo misto linear elíptico, o modelo misto linear com erros nas variáveis e o modelo misto não linear, entre outros. Da mesma forma que no modelo misto linear, o desenvolvimento de métodos de estimação, inferência, técnicas de diagnóstico e ferramentas computacionais tem crescido nos últimos anos.

O método de estimação de máxima verossimilhança restrita tem sido desenvolvido apenas para alguns tipos de modelo. Nesses casos os estimadores dos componentes de variâncias são, em geral, menos tendenciosos do que pelo método de máxima verossimilhança. Demidenko (2013) e Verbeke e Molenberghs (2000) apresentam o método para o modelo misto linear.

Neste capítulo desenvolvemos os procedimentos de estimação de máxima verossimilhança perfilada modificada e restrita para o modelo misto linear elíptico.

\subsection{Modelo misto linear elíptico}

Considerando o modelo proposto por Laird e Ware (1982), temos que

$$
\mathbf{Y}_{i}=\mathbf{X}_{i} \boldsymbol{\beta}+\mathbf{Z}_{i} \mathbf{d}_{i}+\boldsymbol{\epsilon}_{i}, \quad i=1, \cdots, n
$$

em que $\mathbf{Y}_{i}$ é um vetor de $m_{i}$ medidas repetidas do $i$-ésimo sujeito ou grupo, $\mathbf{X}_{i}$ é uma matriz $\left(m_{i} \times p\right)$ de valores das variáveis explicativas, $\boldsymbol{\beta}$ é um vetor de $p$-coeficientes da regressão denominado efeitos fixos, $\mathbf{Z}_{i}$ é uma matriz de planejamento de dimensão $\left(m_{i} \times q\right)$ dos efeitos aleatórios $\mathbf{d}_{i}$, que é um vetor $q$-dimensional que representa os erros entre os sujeitos ou "clusters" e $\boldsymbol{\epsilon}_{i}$ é um vetor $m_{i}$-dimensional de erros.

Em geral, assume-se que as respostas $\mathbf{Y}_{i}$ são independentes, para $i=1, \ldots, n$, e que os efeitos aleatórios $\mathbf{d}_{i}$ e os erros $\boldsymbol{\epsilon}_{i}$ são independentes com médias zero e matrizes de dispersão, $\mathbf{D}=\mathbf{D}(\gamma)$ e $\boldsymbol{\Sigma}_{m_{i}}=\boldsymbol{\Sigma}_{m_{i}}(\boldsymbol{\alpha})$, respectivamente, que representam a variabilidade entre e intragrupo. Os vetores de parâmetros desconhecidos $\boldsymbol{\alpha}$ e $\boldsymbol{\gamma}$ geralmente são de dimensão reduzida. Verbeke e Molenberghs (2000) apresentam algumas estruturas para as matrizes de dispersão que podem ser consideradas 
dependendo do objetivo da aplicação.

Uma forma de tratar este tipo de modelo é através da formulação hierárquica, dada por

- $\mathbf{Y}_{i} \mid \mathbf{d}_{i} \sim N_{m_{i}}\left(\mathbf{X}_{i} \boldsymbol{\beta}+\mathbf{Z}_{i} \mathbf{d}_{i}, \boldsymbol{\Sigma}_{m_{i}}\right)$,

- $\mathbf{d}_{i} \sim N_{q}(\mathbf{0}, \mathbf{D})$.

Assim, temos que a distribuição conjunta de $\left(\mathbf{Y}_{i}^{\top}, \mathbf{d}_{i}^{\top}\right)^{\top}$ fica expressa na forma

$$
\left[\begin{array}{c}
\mathbf{Y}_{i} \\
\mathbf{d}_{i}
\end{array}\right] \sim N_{m_{i}+q}\left(\left[\begin{array}{c}
\mathbf{X}_{i} \boldsymbol{\beta} \\
\mathbf{0}
\end{array}\right],\left[\begin{array}{cc}
\mathbf{Z}_{i} \mathbf{D} \mathbf{Z}_{i}^{\top}+\boldsymbol{\Sigma}_{m_{i}} & \mathbf{Z}_{i} \mathbf{D} \\
\mathbf{D Z}_{i}^{\top} & \mathbf{D}
\end{array}\right]\right)
$$

A inferência clássica geralmente é baseada no modelo marginal, que é obtido facilmente da distribuição conjunta, sendo dado por $\mathbf{Y}_{i} \sim N_{m_{i}}\left(\mathbf{X}_{i} \boldsymbol{\beta} ; \mathbf{Z}_{i} \mathbf{D}_{i} \mathbf{Z}_{i}^{\top}+\boldsymbol{\Sigma}_{m_{i}}\right)$. Quando o propósito da aplicação é fazer predições, é necessário prever os efeitos aleatórios, sendo a inferência realizada sob o modelo condicional

$$
\mathbf{d}_{i} \mid \mathbf{Y}_{i} \sim N_{q}\left(\mathbf{0}+\mathbf{D} \mathbf{Z}_{i}^{\top} \boldsymbol{\Sigma}_{m_{i}}^{-1}\left(\mathbf{Y}_{i}-\mathbf{X}_{i} \boldsymbol{\beta}\right) ; \mathbf{D} \mathbf{Z}_{i}^{\top} \boldsymbol{\Sigma}_{m_{i}}^{-1} \mathbf{Z}_{i} \mathbf{D}\right) .
$$

Assim, para um modelo misto linear elíptico, se consideramos a formulação hierárquica, temos que

- $\mathbf{Y}_{i} \mid \mathbf{d}_{i} \sim E l_{m_{i}}\left(\mathbf{X}_{i} \boldsymbol{\beta}+\mathbf{Z}_{i} \mathbf{d}_{i}, \boldsymbol{\Sigma}_{m_{i}}, g_{c}\right)$,

- $\mathbf{d}_{i} \sim E l_{q}\left(\mathbf{0}, \mathbf{D}, g_{d}\right) \mathrm{e}$

em que $g_{c}(\cdot)$ e $g_{d}(\cdot)$ representam as funções geradoras de densidades das variaveis $\mathbf{Y}_{i} \mid \mathbf{d}_{i}$ e $\mathbf{d}_{i}$, respectivamente, para $i=1, \ldots, n$. Nem mesmo no caso que as funções geradoras de densidades sejam iguais, a distribuição conjunta de $\left(\mathbf{Y}_{i}^{\top}, \mathbf{d}_{i}^{\top}\right)^{\top}$ não é necessariamente elíptica, e portanto dificulta a determinação da distribuição marginal de $\mathbf{Y}_{i}$.

Uma abordagem alternativa para o modelo misto linear elíptico, como foi apresentado por Osorio (2006), seria considerar que a distribuição conjunta $\left(\mathbf{Y}_{i}^{\top}, \mathbf{d}_{i}^{\top}\right)^{\top}$ tem distribuição elíptica, isto é denotado pela forma

$$
\left[\begin{array}{c}
\mathbf{Y}_{i} \\
\mathbf{d}_{i}
\end{array}\right] \sim E l_{m_{i}+q}\left(\left[\begin{array}{c}
\mathbf{X}_{i} \boldsymbol{\beta} \\
\mathbf{0}
\end{array}\right],\left[\begin{array}{cc}
\mathbf{Z}_{i} \mathbf{D} \mathbf{Z}_{i}^{\top}+\mathbf{\Sigma}_{m_{i}} & \mathbf{Z}_{i} \mathbf{D} \\
\mathbf{D} \mathbf{Z}_{i}^{\top} & \mathbf{D}
\end{array}\right], g^{*}\right)
$$

em que $g^{*}(\cdot)$ é a função geradora de densidade associada à distribuição conjunta de $\left(\mathbf{Y}_{i}^{\top}, \mathbf{d}_{i}^{\top}\right)^{\top}$. Considerando as propriedades das distribuições elípticas, dadas no Apêndice $\mathrm{C}$, temos que as distribuições marginais e condicionais também são elípticas mas não necessariamente com a mesma função geradora $g^{*}(\cdot)$. Contudo, esse enfoque não se sustenta para que os efeitos aleatórios $\mathbf{d}_{i}$ e os erros $\boldsymbol{\epsilon}_{i}$ sejam independentes. Somente podemos garantir que não sejam correlacionados, exceto para o caso normal. Lembramos que a distribuição normal é uma distribuição elíptica, assim podemos considerar que o modelo misto linear é um caso particular do modelo misto linear elíptico. Neste trabalho analisaremos os métodos de estimação visto no capítulo anterior utilizando esta última formulação do modelo misto linear elíptico.

\subsection{Métodos de estimação}

Verbeke e Molenberghs (2000) discutem a metodologia de máxima verossimilhança restrita para os componentes de variância em modelos mistos lineares. Os estimadores são obtidos a partir da maximização da função de verossimilhança de um conjunto de contrastes $\mathbf{U}=\mathbf{A}^{\top} \mathbf{Y}$, em que $\mathbf{A}$ é uma matriz $(n \times(n-p))$ de posto completo, com colunas ortogonais às colunas da matriz $\mathbf{X}$. Então, $\mathbf{U}$ tem distribuição normal, que não depende de $\boldsymbol{\beta}$, e a função de verossimilhança resultante pode ser utilizada para estimar os componentes de variância. Verbeke e Molenberghs (2000) mencionam 
que a metodologia é baseada no artigo de Harville (1974).

Da mesma forma, McGilchrist e Yau (1995) propõem o contraste $\mathbf{D}^{-1}-\mathbf{D}^{-1} \mathbf{X}\left(\mathbf{X}^{\top} \mathbf{D}^{-1} \mathbf{X}\right)^{-1} \mathbf{X}^{\top}$ $\mathbf{D}^{-1}$ para um modelo misto linear com estrutura de variância-covariância dada por $\mathbf{D}+\mathbf{Z} \mathbf{A} \mathbf{Z}^{\top}$, assumindo que $\mathbf{D}$ é conhecida e está associada à estrutura de variância-covariância do vetor de erros $\boldsymbol{\epsilon}$. Enquanto que A é uma matriz diagonal por bloco, em que cada matriz da diagonal, $\mathbf{A}_{i}$, é uma estrutura de variância-covariância que depende do vetor de parâmetros $\gamma$ desconhecidos. No mesmo artigo, os autores propõem uma aplicação do método de estimação restrita para modelos mistos lineares generalizados com estrutura de variância-covariância $\mathbf{D}$ dada pela matriz de informação observada. Portanto, utilizam o mesmo contraste do modelo misto linear mas considerando a nova estrutura da matriz D. As especificações do modelo estão dadas no artigo.

Neste capítulo vamos considerar os seguintes métodos de estimação de um modelo linear elíptico, vistos no capítulo anterior: a transformação proposta por Verbyla (1990), o método de estimação de máxima verossimilhança perfilada modificada e aproximada.

Da distribuição conjunta (4.2) obtemos a distribuição marginal $\mathbf{Y}_{i} \sim E l_{m_{i}}\left(\mathbf{X}_{i} \boldsymbol{\beta} ; \mathbf{Z}_{i} \mathbf{D}_{i} \mathbf{Z}_{i}^{\top}+\right.$ $\left.\boldsymbol{\Sigma}_{m_{i}}, g\right), i=1, \ldots, n$. Seja $\boldsymbol{\Sigma}_{i}=\mathbf{Z}_{i} \mathbf{D}_{i} \mathbf{Z}_{i}^{\top}+\boldsymbol{\Sigma}_{m_{i}}$ e $\boldsymbol{\theta}=\left(\boldsymbol{\gamma}^{\top}, \boldsymbol{\alpha}^{\top}\right)^{\top}$ o vetor de parâmetros desconhecidos das matrizes $\boldsymbol{\Sigma}_{i}$ com i=1,..,n, denotaremos por $s$ o total de elementos que contém o vetor $\boldsymbol{\theta}$. Portanto, a função de verossimilhança fica dada por

$$
L(\boldsymbol{\beta}, \boldsymbol{\theta} ; \mathbf{Y})=\prod_{i=1}^{n} L\left(\boldsymbol{\beta}, \boldsymbol{\theta} ; \mathbf{Y}_{i}\right)=\prod_{i=1}^{n}\left|\boldsymbol{\Sigma}_{i}\right|^{-1 / 2} g\left(u_{i}\right)
$$

em que $u_{i}=\left(\mathbf{Y}_{i}-\mathbf{X}_{i} \boldsymbol{\beta}\right)^{\top} \boldsymbol{\Sigma}_{i}^{-1}\left(\mathbf{Y}_{i}-\mathbf{X}_{i} \boldsymbol{\beta}\right)$. Assim, o logaritmo da função de verossimilhança fica expresso na forma

$$
\begin{aligned}
l(\boldsymbol{\beta}, \boldsymbol{\theta} ; \mathbf{Y}) & =\sum_{i=1}^{n} l\left(\boldsymbol{\beta}, \boldsymbol{\theta} ; \mathbf{Y}_{i}\right)=-\frac{1}{2} \sum_{i=1}^{n} \log \left|\boldsymbol{\Sigma}_{i}\right|+\sum_{i=1}^{n} \log g\left(u_{i}\right) \\
& =-\frac{1}{2} \sum_{i=1}^{n} \log \left|\boldsymbol{\Sigma}_{i}\right|+\sum_{i=1}^{n} \log g\left(u_{i}\right) .
\end{aligned}
$$

Em seguida, apresentamos os resultados para as equações de estimação segundo os três métodos.

\subsubsection{Estimação de máxima verossimilhança}

A função escore para $\boldsymbol{\beta}$ fica dada por

$$
\frac{\partial}{\partial \boldsymbol{\beta}} l(\boldsymbol{\beta}, \boldsymbol{\theta} ; \mathbf{Y})=-2 \sum_{i=1}^{n} w_{g}\left(u_{i}\right) \mathbf{X}_{i}^{\top} \boldsymbol{\Sigma}_{i}^{-1}\left(\mathbf{Y}_{i}-\mathbf{X}_{i} \boldsymbol{\beta}\right),
$$

em que $w_{g}\left(u_{i}\right)=d \log \left\{g\left(u_{i}\right)\right\}=g^{\prime}\left(u_{i}\right) / g\left(u_{i}\right)$ e $g^{\prime}\left(u_{i}\right)$ representa a derivada da função $g(\cdot)$.

A função escore para $\theta_{j}, \operatorname{com} j=1, \ldots, s$, fica dada por

$$
\frac{\partial}{\partial \theta_{j}} l(\boldsymbol{\beta}, \boldsymbol{\theta} ; \mathbf{Y})=-\frac{1}{2} \sum_{i=1}^{n} \operatorname{tr}\left\{\boldsymbol{\Sigma}_{i}^{-1} \frac{\partial \boldsymbol{\Sigma}_{i}}{\partial \theta_{j}}\right\}-\sum_{i=1}^{n} w_{g}\left(u_{i}\right)\left[\frac{\partial u_{i}}{\partial \theta_{j}}\right],
$$

em que a derivada parcial de $u_{i}$ com respeito a $\theta_{j}$ fica dada por

$$
\frac{\partial u_{i}}{\partial \theta_{j}}=\left(\mathbf{Y}_{i}-\mathbf{X}_{i} \boldsymbol{\beta}\right)^{\top} \boldsymbol{\Sigma}_{i}^{-1} \frac{\partial \boldsymbol{\Sigma}_{i}}{\partial \theta_{j}} \boldsymbol{\Sigma}_{i}^{-1}\left(\mathbf{Y}_{i}-\mathbf{X}_{i} \boldsymbol{\beta}\right)
$$


para $j=1, \ldots, s$.

A equação de estimação resultante para $\boldsymbol{\beta}$, pode ser escrita como

$$
\boldsymbol{\beta}=\left(\sum_{i=1}^{n} w_{g}\left(u_{i}\right) \mathbf{X}_{i}^{\top} \boldsymbol{\Sigma}_{i}^{-1} \mathbf{X}_{i}\right)^{-1}\left(\sum_{i=1}^{n} w_{g}\left(u_{i}\right) \mathbf{X}_{i}^{\top} \boldsymbol{\Sigma}_{i}^{-1} \mathbf{Y}_{i}\right)
$$

Vemos que a expressão do estimador depende da função geradora através de $w_{g}\left(u_{i}\right)$ e, em geral, esta função não é constante com respeito a $\boldsymbol{\beta}$. Portanto, não temos um estimador explícito para $\boldsymbol{\beta}$. As equações de estimação para $\theta_{j}$ ficam dadas por $\frac{\partial}{\partial \theta_{j}} l(\boldsymbol{\beta}, \boldsymbol{\theta})=0$, em que

$$
-\frac{1}{2} \sum_{i=1}^{n} \operatorname{tr}\left\{\boldsymbol{\Sigma}_{i}^{-1} \frac{\partial \boldsymbol{\Sigma}_{i}}{\partial \theta_{j}}\right\}-\sum_{i=1}^{n} w_{g}\left(u_{i}\right)\left(\mathbf{Y}_{i}-\mathbf{X}_{i} \boldsymbol{\beta}\right)^{\top} \boldsymbol{\Sigma}_{i}^{-1} \frac{\partial \boldsymbol{\Sigma}_{i}}{\partial \theta_{j}} \boldsymbol{\Sigma}_{i}^{-1}\left(\mathbf{Y}_{i}-\mathbf{X}_{i} \boldsymbol{\beta}\right)=0 \text {, }
$$

para $j=1, \ldots, s$. Para cada matriz $\boldsymbol{\Sigma}_{i}$, que depende do vetor $\boldsymbol{\theta}$, não temos um estimador de máxima verossimilhança explícito. Precisaremos de algum método de otimização para encontrar as estimativas de máxima verossimilhança de forma conjunta utilizando o logaritmo da função de verossimilhança dada na equação (4.3) ou resolvendo as equações de estimação dadas em (4.7) para $\boldsymbol{\beta}$ e em (4.8) cada componente do vetor $\boldsymbol{\theta}$. Neste último caso, podemos considerar que na $(r+1)$-iteração a equação para obter a estimativa de $\boldsymbol{\beta}$ fica dada por

$$
\boldsymbol{\beta}_{M V}^{(r+1)}=\left[\sum_{i=1}^{n} w_{g}\left(u_{i}^{(r)}\right) \mathbf{X}_{i}^{\top}\left\{\boldsymbol{\Sigma}_{i M V}^{(r)}\right\}^{-1} \mathbf{X}_{i}\right]^{-1}\left[\sum_{i=1}^{n} w_{g}\left(u_{i}^{(r)}\right) \mathbf{X}_{i}^{\top}\left\{\boldsymbol{\Sigma}_{i M V}^{(r)}\right\}^{-1} \mathbf{Y}_{i}\right]
$$

Neste caso $u_{i}^{(r)}$ é dado por $\left(\mathbf{Y}_{i}-\mathbf{X}_{i} \boldsymbol{\beta}_{M V}^{(r)}\right)^{\top}\left\{\boldsymbol{\Sigma}_{i M V}^{(r)}\right\}^{-1}\left(\mathbf{Y}_{i}-\mathbf{X}_{i} \boldsymbol{\beta}_{M V}^{(r)}\right)$, em que $\boldsymbol{\Sigma}_{i M V}^{(r)}$ denota a $r$ ésima estimativa de máxima verossimilhança da matriz $\boldsymbol{\Sigma} \operatorname{com} r=0, \ldots$

Considerando os resultados obtidos por Lange et al. (1989), Savalli et al. (2006) e Osorio et al. (2007), temos que a matriz de informação de Fisher é uma matriz diagonal por bloco dada por

$$
\mathbf{K}(\boldsymbol{\beta}, \boldsymbol{\theta})=\left[\begin{array}{cc}
\mathbf{K}_{\beta \beta} & \mathbf{0} \\
\mathbf{0} & \mathbf{K}_{\theta \theta}
\end{array}\right]
$$

em que

$$
\mathbf{K}_{\beta \beta}=\sum_{i=1}^{n}\left(\frac{4 d_{g i}}{m_{i}}\right) \mathbf{X}_{i}^{\top} \boldsymbol{\Sigma}_{i}^{-1} \mathbf{X}_{i}
$$

em que $d_{g i}=\mathrm{E}\left\{w_{g}^{2}\left(u_{i}\right) u_{i}\right\}$ e o $(j, k)$-ésimo elemento da matriz $\mathbf{K}_{i, \alpha \alpha}$ fica dado por

$$
\mathbf{K}_{i, \theta_{j} \theta_{k}}=\frac{b_{j, k, i}}{4}\left(\frac{4 f_{g i}}{m_{i}\left(m_{i}+2\right)}-1\right)+\frac{2 f_{g i}}{m_{i}\left(m_{i}+2\right)} \operatorname{tr}\left\{\boldsymbol{\Sigma}_{i}^{-1} \frac{\partial \boldsymbol{\Sigma}_{i}}{\partial \theta_{j}} \boldsymbol{\Sigma}_{i}^{-1} \frac{\partial \boldsymbol{\Sigma}_{i}}{\partial \theta_{k}}\right\},
$$

em que $f_{g i}=\mathrm{E}\left\{w_{g}^{2}\left(u_{i}\right) u_{i}^{2}\right\}$ e $b_{j, k, i}$ é $\operatorname{tr}\left\{\boldsymbol{\Sigma}_{i}^{-1} \frac{\partial \boldsymbol{\Sigma}_{i}}{\partial \theta_{j}}\right\} \operatorname{tr}\left\{\boldsymbol{\Sigma}_{i}^{-1} \frac{\partial \boldsymbol{\Sigma}_{i}}{\partial \theta_{k}}\right\}$, com $j, k$ em $1, \ldots, s$ e para $i=$ $1, \ldots, n$. Uma forma de obter as matrizes de variância e covariância assintóticas para os estimadores de máxima verossimilhança é considerar as inversas das matriz de informação de Fisher.

\subsubsection{Estimação de máxima verossimilhança restrita}

Dado que estamos considerando que os vetores $\mathbf{Y}_{i}, \operatorname{com} i=1, \ldots, n$, são independentes com distribuição elíptica, propomos uma transformação em cada um desses vetores. Portanto, teremos $n$ transformações. Ou seja, temos que $\mathbf{Y}_{i} \sim E l_{m_{i}}\left(\boldsymbol{\mu}_{i}, \boldsymbol{\Sigma}_{i}, g\right)$ com $\boldsymbol{\mu}_{i}=\mathbf{X}_{i} \boldsymbol{\beta}$ e $\boldsymbol{\Sigma}_{i}=\boldsymbol{\Sigma}_{i}(\boldsymbol{\theta})$ e a transformação $\mathbf{W}_{i}^{\top} \mathbf{Y}_{i}$, com $\mathbf{W}_{i}=\left[\mathbf{W}_{i 1}, \mathbf{W}_{i 2}\right]$, em que $\mathbf{W}_{i 1}$ e $\mathbf{W}_{i 2}$ devem seguir as condições

$$
\mathbf{W}_{i 1}^{\top} \mathbf{X}_{i}=\mathbf{I}_{p} \quad e \quad \mathbf{W}_{i 2}^{\top} \mathbf{X}_{i}=\mathbf{0},
$$


para $i=1, \ldots, n$. Lembremos também que precisamos apenas especificar a estrutura da matriz $\mathbf{W}_{i 1}$ e novamente utilizaremos a estrutura proposta por Verbyla (1990), isto é, $\mathbf{W}_{i 1}=\left(\mathbf{X}_{i}^{\top} \mathbf{X}_{i}\right)^{-1} \mathbf{X}_{i}^{\top}$. Assim, todos os resultados apresentados na Seção 3.3 são válidos para cada $\mathbf{Y}_{i}$, com $i=1, \ldots, n$. Então, podemos expressar o logaritmo da função de verossimilhança para os parâmetros $\boldsymbol{\beta}$ e $\boldsymbol{\theta}$ como a soma de cada transformação $\mathbf{W}_{i}^{\top} \mathbf{Y}_{i}$, ou seja

$$
\begin{aligned}
l\left(\boldsymbol{\beta}, \boldsymbol{\theta} ; \mathbf{Y}^{*}\right) & =\sum_{i=1}^{n} l\left(\boldsymbol{\beta}, \boldsymbol{\theta} ; \mathbf{V}_{i 1} \mid \mathbf{V}_{i 2}\right)+l\left(\boldsymbol{\theta} ; \mathbf{V}_{i 2}\right) \\
& =-\frac{1}{2} \sum_{i=1}^{n} \log \left|\boldsymbol{\Sigma}_{i(11.2)}\right|+\sum_{i=1}^{n} \log g_{u_{i 2}}\left(u_{i v}\right)-\frac{1}{2} \sum_{i=1}^{n} \log \left|\mathbf{W}_{i 2}^{\top} \boldsymbol{\Sigma}_{i} \mathbf{W}_{i 2}\right|+\sum_{i=1}^{n} \log g_{i 2}\left(u_{i 2}\right),
\end{aligned}
$$

em que $\mathbf{Y}^{*}$ denota o vetor que contém as transformações $\mathbf{W}_{i}^{\top} \mathbf{Y}_{i}, u_{i 2}$ é dado por $\left(\mathbf{Y}_{i}-\mathbf{X}_{i} \mathbf{b}_{i}\right)^{\top} \boldsymbol{\Sigma}_{i}^{-1}\left(\mathbf{Y}_{i}-\right.$ $\left.\mathbf{X}_{i} \mathbf{b}_{i}\right)$ e $\mathbf{b}_{i}$ denota a expressão $\left(\mathbf{X}_{i}^{\top} \boldsymbol{\Sigma}_{i}^{-1} \mathbf{X}_{i}\right)^{-1} \mathbf{X}_{i}^{\top} \boldsymbol{\Sigma}_{i}^{-1} \mathbf{Y}_{i}, \operatorname{com} i=1, \ldots, n$.

Segundo o método de estimação proposto por Verbyla (1990), devemos maximizar $l\left(\boldsymbol{\beta}, \boldsymbol{\Sigma} ; \mathbf{V}_{1} \mid \mathbf{V}_{2}\right)$ com respeito a $\boldsymbol{\beta}$. Assim, considerando o resultado da equação (3.14), temos que

$$
\begin{aligned}
\frac{\partial}{\partial \boldsymbol{\beta}} \sum_{i=1}^{n} l\left(\boldsymbol{\beta}, \boldsymbol{\theta} ; \mathbf{V}_{i 1} \mid \mathbf{V}_{i 2}\right) & =\sum_{i=1}^{n} \frac{\partial}{\partial \boldsymbol{\beta}} l\left(\boldsymbol{\beta}, \boldsymbol{\theta} ; \mathbf{V}_{i 1} \mid \mathbf{V}_{i 2}\right) \\
& =-2 \sum_{i=1}^{n} w_{g}\left(u_{i}\right)\left(\mathbf{X}_{i}^{\top} \boldsymbol{\Sigma}_{i}^{-1} \mathbf{X}_{i}\right)\left(\mathbf{b}_{i}-\boldsymbol{\beta}\right) .
\end{aligned}
$$

Vemos que esta última expressão é igual à equação de estimação de $\boldsymbol{\beta}$ obtida pelo método de máxima verossimilhança, equação (4.4), dado que o termo $\left(\mathbf{X}_{i}^{\top} \boldsymbol{\Sigma}_{i}^{-1} \mathbf{X}_{i}\right) \mathbf{b}_{i}$ é igual a $\mathbf{X}_{i}^{\top} \boldsymbol{\Sigma}_{i}^{-1} \mathbf{Y}_{i}$ para todo $i=1, \ldots, n$. Portanto, a equação para determinar o estimador para $\boldsymbol{\beta}$ fica dada por

$$
\boldsymbol{\beta}=\left\{\sum_{i=1}^{n} w_{g}\left(u_{i}\right) \mathbf{X}_{i}^{\top} \boldsymbol{\Sigma}_{i}^{-1} \mathbf{X}_{i}\right\}^{-1}\left\{\sum_{i=1}^{n} w_{g}\left(u_{i}\right) \mathbf{X}_{i}^{\top} \boldsymbol{\Sigma}_{i}^{-1} \mathbf{Y}_{i}\right\} .
$$

Portanto, esta última expressão é igual à equação resultante para o método de máxima verossimilhança, equação (4.7), mas que dependem da estimativa do vetor $\boldsymbol{\theta}$ que neste caso será determinado pela maximização de $\sum_{i=1}^{n} l\left(\boldsymbol{\theta} ; \mathbf{V}_{i 2}\right)$ com respeito de $\theta_{j}$, obtendo

$$
\begin{aligned}
\frac{\partial}{\partial \theta_{j}} \sum_{i=1}^{n} l\left(\boldsymbol{\theta} ; \mathbf{V}_{i 2}\right)= & -\frac{1}{2} \sum_{i=1}^{n} \operatorname{tr}\left\{\boldsymbol{\Sigma}_{i}^{-1} \frac{\partial \boldsymbol{\Sigma}_{i}}{\partial \theta_{j}}\right\}-\sum_{i=1}^{n} w_{i 2}\left(u_{i 2}\right)\left[\frac{\partial u_{i 2}}{\partial \theta_{j}}\right] \\
& -\frac{1}{2} \sum_{i=1}^{n} \operatorname{tr}\left\{\left(\mathbf{X}_{i}^{\top} \boldsymbol{\Sigma}_{i}^{-1} \mathbf{X}_{i}\right)^{-1} \mathbf{X}_{i}^{\top} \boldsymbol{\Sigma}_{i}^{-1} \frac{\partial \boldsymbol{\Sigma}_{i}}{\partial \theta_{j}} \boldsymbol{\Sigma}_{i}^{-1} \mathbf{X}_{i}\right\}
\end{aligned}
$$

em que a derivada parcial de $u_{i 2}$ com respeito a $\theta_{j}$ fica dada por

$$
\frac{\partial u_{i 2}}{\partial \theta_{j}}=\left(\mathbf{Y}_{i}-\mathbf{X}_{i} \mathbf{b}_{i}\right)^{\top}\left\{2 \boldsymbol{\Sigma}_{i}^{-1} \mathbf{X}_{i}\left(\mathbf{X}_{i}^{\top} \boldsymbol{\Sigma}_{i}^{-1} \mathbf{X}_{i}\right)^{-1} \mathbf{X}_{i}^{\top}+\mathbf{I}_{m_{i}}\right\} \boldsymbol{\Sigma}_{i}^{-1} \frac{\partial \boldsymbol{\Sigma}_{i}}{\partial \theta_{j}} \boldsymbol{\Sigma}_{i}^{-1}\left(\mathbf{Y}_{i}-\mathbf{X}_{i} \mathbf{b}_{i}\right)
$$

para $j=1, \ldots, s$ e lembrando que $u_{i 2}=\left(\mathbf{Y}_{i}-\mathbf{X}_{i} \mathbf{b}_{i}\right)^{\top} \boldsymbol{\Sigma}_{i}^{-1}\left(\mathbf{Y}_{i}-\mathbf{X}_{i} \mathbf{b}_{i}\right)$, com $i=1, \ldots, n$. Vemos que o estimador da matriz de escala não tem forma explícita e deve ser obtido com base em algum método de aproximação. Assim, obtemos estimativas de máxima verossimilhança restrita para cada $\boldsymbol{\Sigma}_{i}$; denotando $\widehat{\boldsymbol{\Sigma}}_{i M V R}$. Por conseguinte, para encontrar uma estimativa para $\boldsymbol{\beta}$ resolvemos iterativamente a seguinte equação:

$$
\boldsymbol{\beta}_{M V R}^{(r+1)}=\left\{\sum_{i=1}^{n} w_{g}\left(u_{i}^{(r)}\right) \mathbf{X}_{i}^{\top} \widehat{\boldsymbol{\Sigma}}_{i M V R}^{-1} \mathbf{X}_{i}\right\}^{-1}\left\{\sum_{i=1}^{n} w_{g}\left(u_{i}^{(r)}\right) \mathbf{X}_{i}^{\top} \widehat{\boldsymbol{\Sigma}}_{i M V R}^{-1} \mathbf{Y}_{i}\right\} .
$$


Neste caso, $u_{i}^{(r)}$ é dado por $\left(\mathbf{Y}_{i}-\mathbf{X}_{i} \boldsymbol{\beta}_{M V R}^{(r)}\right)^{\top} \widehat{\boldsymbol{\Sigma}}_{i M V R}^{-1}\left(\mathbf{Y}_{i}-\mathbf{X}_{i} \boldsymbol{\beta}_{M V R}^{(r)}\right)$ para $r=0 \ldots$

\subsubsection{Estimação baseada na verossimilhança perfilada modificada}

Consideraremos os resultados do Capítulo 3 (Seção 3.4) em que vimos o método da verossimilhança perfilada modificada para o modelo linear elíptico. Temos que o logaritmo da função de verossimilhança perfilada de $\boldsymbol{\theta}$ para o modelo misto linear elíptico fica dado por

$$
\begin{aligned}
l_{p}(\boldsymbol{\theta}) & =-\frac{1}{2} \sum_{i=1}^{n} \log \left|\boldsymbol{\Sigma}_{i}\right|+\sum_{i=1}^{n} \log g\left(\hat{u}_{i}\right) \\
& =-\frac{1}{2} \sum_{i=1}^{n} \log \left|\boldsymbol{\Sigma}_{i}\right|+\sum_{i=1}^{n} \log g\left\{\left(\mathbf{Y}_{i}-\mathbf{X}_{i} \hat{\boldsymbol{\beta}}_{\theta}\right)^{\top} \boldsymbol{\Sigma}_{i}^{-1}\left(\mathbf{Y}_{i}-\mathbf{X}_{i} \hat{\boldsymbol{\beta}}_{\theta}\right)\right\} .
\end{aligned}
$$

O termo $\hat{\boldsymbol{\beta}}_{\theta}$ representa o estimador de máxima verossimilhança parcial de $\boldsymbol{\beta}$ para $\boldsymbol{\theta}$ fixado.

\section{Ajuste da função de verossimilhança perfilada modificada}

Para obter a função de verossimilhança perfilada modificada precisamos determinar a matriz de informação observada com respeito a $\boldsymbol{\beta}$. Esta pode ser obtida da segunda derivada da função de verossimilhança com respeito a $\boldsymbol{\beta}$. Assim, derivando a equação (4.4) temos que

$\frac{\partial}{\partial \boldsymbol{\beta} \partial \boldsymbol{\beta}^{\top}} l(\boldsymbol{\beta}, \boldsymbol{\theta} ; \mathbf{Y})=4 \sum_{i=1}^{n} w_{g}^{\prime}\left(u_{i}\right) \mathbf{X}_{i}^{\top} \boldsymbol{\Sigma}_{i}^{-1}\left(\mathbf{Y}_{i}-\mathbf{X}_{i} \boldsymbol{\beta}\right)\left(\mathbf{Y}_{i}-\mathbf{X} \boldsymbol{\beta}\right)^{\top} \boldsymbol{\Sigma}_{i}^{-1} \mathbf{X}_{i}+2 \sum_{i=1}^{n} w_{g}\left(u_{i}\right) \mathbf{X}_{i}^{\top} \boldsymbol{\Sigma}_{i}^{-1} \mathbf{X}_{i}$,

em que $w_{g}^{\prime}\left(u_{i}\right)$ denota a segunda derivada da função $\log \left\{g\left(u_{i}\right)\right\}$. A matriz de informação observada com respeito a $\boldsymbol{\beta}$ avaliada no estimador de máxima verossimilhança parcial de $\boldsymbol{\beta}$, para o vetor $\boldsymbol{\theta}$ fixado, fica dada por

$$
\begin{aligned}
\mathbf{J}_{\beta \beta}\left(\hat{\boldsymbol{\beta}}_{\theta}, \boldsymbol{\theta}\right)= & 4 \sum_{i=1}^{n} w_{g}^{\prime}\left(\hat{u}_{i \theta}\right) \mathbf{X}_{i}^{\top} \boldsymbol{\Sigma}_{i}^{-1}\left(\mathbf{Y}_{i}-\mathbf{X}_{i} \hat{\boldsymbol{\beta}}_{\theta}\right)\left(\mathbf{Y}_{i}-\mathbf{X} \hat{\boldsymbol{\beta}}_{\theta}\right)^{\top} \boldsymbol{\Sigma}_{i}^{-1} \mathbf{X}_{i}+2 \sum_{i=1}^{n} w_{g}\left(\hat{u}_{i \theta}\right) \mathbf{X}_{i}^{\top} \boldsymbol{\Sigma}_{i}^{-1} \mathbf{X}_{i} \\
= & 2 \sum_{i=1}^{n} \mathbf{X}_{i}^{\top} \boldsymbol{\Sigma}_{i}^{-1 / 2}\left\{2 w_{g}^{\prime}\left(\hat{u}_{i \theta}\right) \boldsymbol{\Sigma}_{i}^{-1 / 2}\left(\mathbf{Y}_{i}-\mathbf{X}_{i} \hat{\boldsymbol{\beta}}_{\theta}\right)\left(\mathbf{Y}_{i}-\mathbf{X} \hat{\boldsymbol{\beta}}_{\theta}\right)^{\top} \boldsymbol{\Sigma}_{i}^{-1 / 2}\right. \\
& \left.+w_{g}\left(\hat{u}_{i \theta}\right) \mathbf{I}_{m_{i}}\right\} \boldsymbol{\Sigma}_{i}^{-1 / 2} \mathbf{X}_{i} \\
\equiv & 2 C_{g m}(\boldsymbol{\theta}),
\end{aligned}
$$

em que a expressão $\hat{u}_{i \theta}$ é dada por $\left(\mathbf{Y}_{i}-\mathbf{X}_{i} \hat{\boldsymbol{\beta}}_{\theta}\right)^{\top} \boldsymbol{\Sigma}^{-1}\left(\mathbf{Y}_{i}-\mathbf{X}_{i} \hat{\boldsymbol{\beta}}_{\theta}\right)$, para $i=1, \ldots, n$. Portanto, usando a equação (4.12) o ajuste da função de verossimilhança perfilada modificada de $\boldsymbol{\theta}$ fica dado por

$$
\begin{aligned}
l_{p m}^{*}(\boldsymbol{\theta}) & =l_{p}(\boldsymbol{\theta})-\frac{1}{2} \log \left\{\left|\mathbf{J}_{\beta \beta}\left(\hat{\boldsymbol{\beta}}_{\theta}, \boldsymbol{\theta}\right)\right|\right\} \\
& =l_{p}(\boldsymbol{\theta})-\frac{1}{2} \log \{2 p\}-\frac{1}{2} \log \left\{\left|C_{g m}(\boldsymbol{\theta})\right|\right\}
\end{aligned}
$$

A derivada desta ùltima expressão com respeito ao $j$-ésimo elemento de $\boldsymbol{\theta}$ fica dada por

$$
\frac{\partial}{\partial \theta_{j}} l_{p m}^{*}(\theta)=-\frac{1}{2} \sum_{i=1}^{n} \operatorname{tr}\left\{\boldsymbol{\Sigma}_{i}^{-1} \frac{\partial \boldsymbol{\Sigma}_{i}}{\partial \theta_{j}}\right\}+\sum_{i=1}^{n} w_{g}\left(\hat{u}_{i \theta}\right)\left[\frac{\partial \hat{u}_{i \theta}}{\partial \theta_{j}}\right]-\frac{1}{2} \operatorname{tr}\left\{C_{g m}(\boldsymbol{\theta})^{-1} \frac{\partial}{\partial \theta_{j}}\left[C_{g m}(\boldsymbol{\theta})\right]\right\},
$$


enquanto que a derivada de $C_{g m}(\boldsymbol{\theta})$ com respeito a $\theta_{j}$ é dada por

$$
\begin{aligned}
\frac{\partial}{\partial \theta_{j}} C_{g m}(\boldsymbol{\theta}) & =2 \sum_{i=1}^{n} w_{g}^{\prime \prime}\left(\hat{u}_{i \theta}\right)\left[\frac{\partial \hat{u}_{i \theta}}{\partial \theta_{j}}\right] \mathbf{X}_{i}^{\top} \boldsymbol{\Sigma}_{i}^{-1}\left(\mathbf{Y}_{i}-\mathbf{X}_{i} \hat{\boldsymbol{\beta}}_{\theta}\right)\left(\mathbf{Y}_{i}-\mathbf{X} \hat{\boldsymbol{\beta}}_{\theta}\right)^{\top} \boldsymbol{\Sigma}_{i}^{-1} \mathbf{X}_{i} \\
& +4 \sum_{i=1}^{n} w_{g}^{\prime}\left(\hat{u}_{i \theta}\right) \frac{\partial}{\partial \theta_{j}}\left[\mathbf{X}_{i}^{\top} \boldsymbol{\Sigma}_{i}^{-1}\left(\mathbf{Y}_{i}-\mathbf{X}_{i} \hat{\boldsymbol{\beta}}_{\theta}\right)\right]\left(\mathbf{Y}_{i}-\mathbf{X} \hat{\boldsymbol{\beta}}_{\theta}\right)^{\top} \boldsymbol{\Sigma}_{i}^{-1} \mathbf{X}_{i} \\
& +\sum_{i=1}^{n} w_{g}^{\prime}\left(\hat{u}_{i \theta}\right)\left[\frac{\partial \hat{u}_{i \theta}}{\partial \theta_{j}}\right] \mathbf{X}_{i}^{\top} \boldsymbol{\Sigma}_{i}^{-1} \mathbf{X}_{i}+\sum_{i=1}^{n} w_{g}\left(\hat{u}_{i \theta}\right) \frac{\partial}{\partial \theta_{j}}\left[\mathbf{X}_{i}^{\top} \boldsymbol{\Sigma}_{i}^{-1} \mathbf{X}_{i}\right]
\end{aligned}
$$

em que

$$
\frac{\partial}{\partial \theta_{j}}\left[\mathbf{X}_{i}^{\top} \boldsymbol{\Sigma}_{i}^{-1}\left(\mathbf{Y}_{i}-\mathbf{X}_{i} \hat{\boldsymbol{\beta}}_{\theta}\right)\right]=-\mathbf{X}_{i}^{\top} \boldsymbol{\Sigma}_{i}^{-1}\left[\frac{\partial \boldsymbol{\Sigma}_{i}}{\partial \theta_{j}}\right] \boldsymbol{\Sigma}_{i}^{-1}\left(\mathbf{Y}_{i}-\mathbf{X}_{i} \hat{\boldsymbol{\beta}}_{\theta}\right)-\mathbf{X}_{i}^{\top} \boldsymbol{\Sigma}_{i}^{-1} \mathbf{X}_{i}\left[\frac{\partial \hat{\boldsymbol{\beta}}_{\theta}}{\partial \theta_{j}}\right]
$$

e

$$
\frac{\partial \hat{u}_{i \theta}}{\partial \theta_{j}}=-2 \mathbf{X}_{i}^{\top} \boldsymbol{\Sigma}_{i}^{-1} \mathbf{X}_{i}\left[\frac{\partial \hat{\boldsymbol{\beta}}_{\theta}}{\partial \theta_{j}}\right]\left(\mathbf{Y}_{i}-\mathbf{X}_{i} \hat{\boldsymbol{\beta}}_{\theta}\right)-\left(\mathbf{Y}_{i}-\mathbf{X}_{i} \hat{\boldsymbol{\beta}}_{\theta}\right)^{\top} \boldsymbol{\Sigma}_{i}^{-1}\left[\frac{\partial \boldsymbol{\Sigma}_{i}}{\partial \theta_{j}}\right] \boldsymbol{\Sigma}_{i}^{-1}\left(\mathbf{Y}_{i}-\mathbf{X}_{i} \hat{\boldsymbol{\beta}}_{\theta}\right)
$$

para $i=1, \ldots, n$ e $j=1, \ldots, s$. A derivada parcial de $\hat{\boldsymbol{\beta}}_{\theta}$ com respeito a $\theta_{j}$ não pode ser calculada de forma exata pois não temos uma expressão explícita para $\hat{\boldsymbol{\beta}}_{\theta}$. Na equação (4.9) apresentamos uma expressão para calcular o estimador de máxima verossimilhança de $\boldsymbol{\beta}$.

Em conclusão, para obter as estimativas para este método de estimação teremos que resolver um sistema de equações de $\hat{\boldsymbol{\beta}}_{\theta}$ e substituir na função de verossimilhança perfilada modificada para $\operatorname{logo}$ maximizar esta função com respeito ao vetor $\boldsymbol{\theta}$. Uma vez obtida a estimativa de $\boldsymbol{\theta}$ podemos encontrar a estimativa para $\boldsymbol{\beta}$ resolvendo iterativamente a equação

$$
\boldsymbol{\beta}_{M V P M}^{(r+1)}=\left\{\sum_{i=1}^{n} w_{g}\left(\hat{u}_{i}^{(r)}\right) \mathbf{X}_{i}^{\top} \widehat{\boldsymbol{\Sigma}}_{i M V P M}^{-1} \mathbf{X}_{i}\right\}^{-1}\left\{\sum_{i=1}^{n} w_{g}\left(\hat{u}_{i}^{(r)}\right) \mathbf{X}_{i}^{\top} \widehat{\boldsymbol{\Sigma}}_{i P M}^{-1} \mathbf{Y}_{i}\right\}
$$

Neste caso, $\hat{u}_{i}^{(r)}$ é dado por $\left(\mathbf{Y}_{i}-\mathbf{X}_{i} \boldsymbol{\beta}_{M V P M}^{(r)}\right)^{\top} \widehat{\boldsymbol{\Sigma}}_{i M V P M}^{-1}\left(\mathbf{Y}_{i}-\mathbf{X}_{i} \boldsymbol{\beta}_{M V P M}^{(r)}\right)$ para $r=0 \ldots$

\section{Ajuste da função de verossimilhança perfilada modificada aproximada}

Para determinar a função de verossimilhança perfilada modificada aproximada precisamos obter a matriz de informação esperada. Da mesma forma que na Seção 3.4.2, utilizaremos os resultados apresentados por Lange et al. (1989) com respeito à matriz de informação esperada de uma distribuição elíptica para obtermos as derivadas com respeito a dois elementos de $\boldsymbol{\beta}$, sejam $\beta_{k}$ e $\beta_{l}$ com $k \neq l$. Consideraremos a variável $\mathbf{z}_{i}$ como $\mathbf{z}_{i}=\boldsymbol{\Sigma}_{i}^{-1 / 2}\left(\mathbf{Y}_{i}-\mathbf{X}_{i} \boldsymbol{\beta}\right)$, para $i=1, \ldots, n$ e $\mathbf{X}_{i k}$ como sendo a k-ésima coluna da matriz $\mathbf{X}_{i}$ e $\mathbf{X}_{i l}$ a l-ésima coluna da matriz $\mathbf{X}_{i}$. Então, obtemos

$$
\begin{aligned}
\frac{\partial l\left(\boldsymbol{\beta}, \boldsymbol{\theta} ; \mathbf{Y}_{i}\right)}{\partial \beta_{k}} \frac{\partial l\left(\boldsymbol{\beta}, \boldsymbol{\theta} ; \mathbf{Y}_{i}\right)}{\partial \beta_{l}} & =\left\{-2 w\left(u_{i}\right) \mathbf{X}_{i k}^{\top} \boldsymbol{\Sigma}_{i}^{-1}\left(\mathbf{Y}_{i}-\mathbf{X}_{i} \boldsymbol{\beta}\right)\right\}\left\{-2 w\left(u_{i}\right) \mathbf{X}_{i l}^{\top} \boldsymbol{\Sigma}_{i}^{-1}\left(\mathbf{Y}_{i}-\mathbf{X}_{i} \boldsymbol{\beta}\right)\right\} \\
& =4 w_{g}^{2}\left(u_{i}\right)\left(\mathbf{Y}_{i}-\mathbf{X}_{i} \boldsymbol{\beta}\right)^{\top} \boldsymbol{\Sigma}_{i}^{-1} \mathbf{X}_{i k} \mathbf{X}_{i l}^{\top} \boldsymbol{\Sigma}_{i}^{-1}\left(\mathbf{Y}_{i}-\mathbf{X}_{i} \boldsymbol{\beta}\right) \\
& =4 w_{g}^{2}\left(u_{i}\right) \mathbf{z}_{i}^{\top} \boldsymbol{\Sigma}_{i}^{-1 / 2} \mathbf{X}_{i k} \mathbf{X}_{i l}^{\top} \boldsymbol{\Sigma}_{i}^{-1 / 2} \mathbf{z}_{i},
\end{aligned}
$$

para $i=1, \ldots, n$ e $k, l=1, \ldots, p$. Para encontrarmos a matriz de informação esperada, consideramos $\left\|\mathbf{z}_{i}\right\|^{2}=\mathbf{z}_{i}^{\top} \mathbf{z}_{i}=\left(\mathbf{Y}_{i}-\mathbf{X}_{i} \boldsymbol{\beta}_{i}\right)^{\top} \boldsymbol{\Sigma}_{i}^{-1 / 2} \boldsymbol{\Sigma}_{i}^{-1 / 2}\left(\mathbf{Y}_{i}-\mathbf{X}_{i} \boldsymbol{\beta}\right)$. Assim, conforme mostrado por Lange $e t$ al. 
(1989) segue que

$$
\mathrm{E}\left\{\frac{\partial l\left(\boldsymbol{\beta}, \boldsymbol{\theta} ; \mathbf{Y}_{i}\right)}{\partial \beta_{k}} \frac{\partial l\left(\boldsymbol{\beta}, \boldsymbol{\theta} ; \mathbf{Y}_{i}\right)}{\partial \beta_{l}}\right\}=\frac{4}{m_{i}} \mathrm{E}\left\{\left\|\mathbf{z}_{i}\right\|^{2} w_{g}^{2}\left(u_{i}\right)\right\} \mathbf{X}_{i l}^{\top} \boldsymbol{\Sigma}_{i}^{-1} \mathbf{X}_{i k} .
$$

Então, a matriz de informação esperada avaliada no estimador de $\boldsymbol{\beta}$ fica dada por

$$
\mathbf{K}_{\beta \beta}\left(\hat{\boldsymbol{\beta}}_{\theta}, \boldsymbol{\theta}\right)=\left.\sum_{i=1}^{n} \frac{4}{m_{i}} \mathrm{E}\left\{\left\|\mathbf{z}_{i}\right\|^{2} w_{g}^{2}\left(u_{i}\right)\right\}\right|_{\beta=\hat{\beta}_{\theta}} \mathbf{X}_{i}^{\top} \boldsymbol{\Sigma}_{i}^{-1} \mathbf{X}_{i} .
$$

Vemos que esta última expressão depende da função geradora, através de $w_{g}^{2}\left(u_{i}\right)$.

O ajuste da função de verossimilhança perfilada modificada de $\boldsymbol{\theta}$ fica dado por

$$
\begin{aligned}
l_{p m a}^{*}(\boldsymbol{\theta}) & =l_{p}(\boldsymbol{\theta})-\frac{1}{2} \log \left|\mathbf{K}_{\beta \beta}\left(\hat{\boldsymbol{\beta}}_{\theta}, \boldsymbol{\theta}\right)\right| \\
& =l_{p}(\boldsymbol{\theta})-\frac{1}{2} \log \left|\frac{1}{2 \pi} \sum_{i=1}^{n} \frac{4}{m_{i}} \mathrm{E}\left\{\left\|\mathbf{z}_{i}\right\|^{2} w_{g}^{2}\left(u_{i}\right)\right\}\right|_{\beta=\hat{\beta}_{\theta}} \mathbf{X}_{i}^{\top} \boldsymbol{\Sigma}_{i}^{-1} \mathbf{X}_{i} \mid .
\end{aligned}
$$

O estimador de $\boldsymbol{\beta}$ é obtido da mesma forma que o estimador de máxima verossimilhança $\left(\hat{\boldsymbol{\beta}}_{M V}\right)$ mas dependerá da estimativa de $\boldsymbol{\Sigma}$ que será calculada da maximização de (4.14) com respeito a $\theta_{j}$. Aqui, diferente da equação (3.31), não podemos decompor o termo da matriz de informação esperada de $\boldsymbol{\beta}$ avaliada em $\hat{\boldsymbol{\beta}}_{\theta}$, pois temos o determinante de uma soma. Logo,

$$
\begin{aligned}
\frac{\partial}{\partial \theta_{j}} l_{p m a}^{*}(\boldsymbol{\theta})= & -\frac{1}{2} \sum_{i=1}^{n} \operatorname{tr}\left\{\boldsymbol{\Sigma}_{i}^{-1} \frac{\partial \boldsymbol{\Sigma}_{i}}{\partial \theta_{j}}\right\}-\sum_{i=1}^{n} w_{g}\left(\hat{u}_{i \theta}\right)\left[\frac{\partial \hat{u}_{i \theta}}{\partial \theta_{j}}\right] \\
- & \operatorname{tr}\left\{\left.\frac{1}{2 \pi} \sum_{i=1}^{n} \frac{4}{m_{i}} \mathrm{E}\left\{\left\|\mathbf{z}_{i}\right\|^{2} w_{g}^{2}\left(u_{i}\right)\right\}\right|_{\beta=\hat{\beta}_{\theta}} \mathbf{X}_{i}^{\top} \boldsymbol{\Sigma}_{i}^{-1} \mathbf{X}_{i}\right\} \\
& \times\left(\left.\frac{1}{2 \pi} \sum_{i=1}^{n} \frac{4}{m_{i}} \mathrm{E}\left\{\left\|\mathbf{z}_{i}\right\|^{2} w_{g}^{2}\left(u_{i}\right)\right\}\right|_{\beta=\hat{\beta}_{\theta}} \mathbf{X}_{i}^{\top} \boldsymbol{\Sigma}_{i}^{-1} \frac{\partial \boldsymbol{\Sigma}_{i}}{\partial \theta_{j}} \boldsymbol{\Sigma}_{i}^{-1} \mathbf{X}_{i}\right),
\end{aligned}
$$

em que a derivada parcial de $\hat{u}_{i \theta}$ como respeito a $\theta_{j}$ foi dada na equação (4.13), para $j=1, \ldots, s$. Vemos que a expressão resultante é mais complexa do que no caso do modelo linear elíptico considerado no capítulo anterior. Portanto, para obter as estimativas de máxima verossimilhança perfilada modificada aproximada precisamos definir um processo iterativo de estimação. Assim, da mesma forma que o método anterior, o processo iterativo para obter a estimativa de $\boldsymbol{\beta}$ fica dado por

$$
\boldsymbol{\beta}_{M V P M A}^{(r+1)}=\left\{\sum_{i=1}^{n} w_{g}\left(\hat{u}_{i}^{(r)}\right) \mathbf{X}_{i}^{\top} \widehat{\boldsymbol{\Sigma}}_{i M V P M A}^{-1} \mathbf{X}_{i}\right\}^{-1}\left\{\sum_{i=1}^{n} w_{g}\left(\hat{u}_{i}^{(r)}\right) \mathbf{X}_{i}^{\top} \widehat{\boldsymbol{\Sigma}}_{i M V P M A}^{-1} \mathbf{Y}_{i}\right\}
$$

em que $\hat{u}_{i}^{(r)}$ é dado por $\left(\mathbf{Y}_{i}-\mathbf{X}_{i} \boldsymbol{\beta}_{M V P M A}^{(r)}\right)^{\top} \widehat{\boldsymbol{\Sigma}}_{i M V P M A}^{-1}\left(\mathbf{Y}_{i}-\mathbf{X}_{i} \boldsymbol{\beta}_{M V P M A}^{(r)}\right)$, para $r=0 \ldots$

Até agora vimos que para todos os métodos de estimação a equação para determinar as estimativas de $\boldsymbol{\beta}$ tem a mesma estrutura. Essa estrutura fica dependendo da função $g(\cdot)$ mediante a função $w_{g}(\cdot)$. Portanto, a expressão da equação de estimação de $\boldsymbol{\beta}$ mudará dependendo da distribuição elíptica que seja analisada. No caso mais simples, temos o modelo misto linear normal, em que $w_{g}(\cdot)=-1 / 2$, a equação de estimação de $\boldsymbol{\beta}$ torna-se uma expressão explícita com respeito a $\boldsymbol{\beta}$ e fica dada por

$$
\hat{\boldsymbol{\beta}}=\left(\sum_{i=1}^{n} \mathbf{X}_{i}^{\top} \widehat{\boldsymbol{\Sigma}}_{i}^{-1} \mathbf{X}_{i}\right)^{-1}\left(\sum_{i=1}^{n} \mathbf{X}_{i}^{\top} \widehat{\boldsymbol{\Sigma}}_{i}^{-1} \mathbf{Y}_{i}\right)
$$


Já para o modelo misto linear t-Student, a função $w_{g}(\cdot)$ não é constate com respeito a $\boldsymbol{\beta}$, isto torna mais complexo o processo de estimação. Na próxima seção analisaremos os métodos de estimação para este modelo e para o modelo misto linear exponencial potência.

\subsection{Casos particulares de distribuições elípticas}

Nesta seção especificamos os métodos de estimação para dois distribuições que pertence à família elíptica. Assim, desenvolvemos os métodos vistos na seção anterior para o modelo misto linear tStudent e o modelo misto linear exponencial potência.

\subsubsection{Modelo misto linear t-Student}

Consideraremos $\mathbf{Y}_{1}, \ldots, \mathbf{Y}_{n}$ uma amostra aleatória em que $\mathbf{Y}_{i}$ com $i=1, \ldots, n$ é um vetor aleatório e o modelo apresentado na equação (4.1). Da mesma forma que foi apresentada na Tese de Doutorado de Osorio (2006), consideramos que a distribuição conjunta $\left(\mathbf{Y}_{i}^{\top}, \mathbf{d}_{i}^{\top}\right)^{\top}$ tem distribuição t-Student $\operatorname{com} \nu$ graus de liberdade, tal que

$$
\left[\begin{array}{c}
\mathbf{Y}_{i} \\
\mathbf{d}_{i}
\end{array}\right] \sim t_{m_{i}+q}\left\{\left[\begin{array}{c}
\mathbf{X}_{i} \boldsymbol{\beta} \\
\mathbf{0}
\end{array}\right],\left[\begin{array}{ccc}
\mathbf{Z}_{i} \mathbf{D}_{i} \mathbf{Z}_{i}^{\top}+\mathbf{\Sigma}_{m_{i}} & \mathbf{Z}_{i} \mathbf{D}_{i} & \boldsymbol{\Sigma}_{m_{i}} \\
\mathbf{D}_{i} \mathbf{Z}_{i}^{\top} & \mathbf{D}_{i} & \mathbf{0}
\end{array}\right], \nu\right\} .
$$

Então, por propriedade da distribuição t-Student multivariada temos que a distribuição marginal $\mathbf{Y}_{i} \sim t_{m_{i}}\left(\mathbf{X}_{i} \boldsymbol{\beta}, \boldsymbol{\Sigma}_{i}, \nu\right)$, em que $\boldsymbol{\Sigma}_{i}=\mathbf{Z}_{i} \mathbf{D}_{i} \mathbf{Z}_{i}^{\top}+\boldsymbol{\Sigma}_{m_{i}}$ e $\boldsymbol{\theta}=\left(\boldsymbol{\gamma}^{\top}, \boldsymbol{\alpha}^{\top}\right)^{\top}$ é o vetor de dimensão $s$, que contém os parâmetros desconhecidos de todas as matrizes $\boldsymbol{\Sigma}_{i}$, com $i=1, \ldots, n$. O parâmetro $\nu$ representa os graus de liberdade que assumiremos conhecido. Portanto, a função de verossimilhança é dada por

$$
L(\boldsymbol{\beta}, \boldsymbol{\theta} ; \mathbf{Y})=\prod_{i=1}^{n} L\left(\boldsymbol{\beta}, \boldsymbol{\theta} ; \mathbf{Y}_{i}\right)=\prod_{i=1}^{n}\left|\boldsymbol{\Sigma}_{i}\right|^{-1 / 2} c_{2 i}\left(1+u_{i} / \nu\right)^{-\left(\nu+m_{i}\right) / 2},
$$

em que $u_{i}$ é a distância dada por $\left(\mathbf{Y}_{i}-\mathbf{X}_{i} \boldsymbol{\beta}\right)^{\top} \boldsymbol{\Sigma}_{i}^{-1}\left(\mathbf{Y}_{i}-\mathbf{X}_{i} \boldsymbol{\beta}\right)$. O parâmetro $\nu$ será assumido conhecido e portanto o termo $c_{2 i}=\frac{\Gamma\left(\left(\nu+m_{i}\right) / 2\right)}{(\pi \nu)^{m_{i} / 2} \Gamma(\nu / 2)}$ não depende de parâmetros desconhecidos. $\mathrm{O}$ logaritmo da função de verossimilhança fica expresso na forma

$$
\begin{aligned}
l(\boldsymbol{\beta}, \boldsymbol{\theta} ; \mathbf{Y})=\sum_{i=1}^{n} l\left(\boldsymbol{\beta}, \boldsymbol{\theta} ; \mathbf{Y}_{i}\right) & =-\frac{1}{2} \sum_{i=1}^{n} \log \left|\boldsymbol{\Sigma}_{i}\right|+\sum_{i=1}^{n} \log c_{2 i}+\sum_{i=1}^{n} \log \left(1+u_{i} / \nu\right)^{-\left(\nu+m_{i}\right) / 2} \\
& =c t e-\frac{1}{2} \sum_{i=1}^{n} \log \left|\boldsymbol{\Sigma}_{i}\right|-\sum_{i=1}^{n} \frac{\nu+m_{i}}{2} \log \left(1+u_{i} / \nu\right) .
\end{aligned}
$$

Como vimos na seção anterior a estrutura da equação para estimar $\boldsymbol{\beta}$ é a mesma para todos os métodos. Então, para o modelo misto linear t-Student a fórmula geral das equações de estimação de $\boldsymbol{\beta}$ pode ser expressa pelo processo iterativo

$$
\boldsymbol{\beta}_{M}^{(r+1)}=\left[\sum_{i=1}^{n} \frac{\nu+m_{i}}{\nu+u_{i M}^{(r)}} \mathbf{X}_{i}^{\top}\left\{\boldsymbol{\Sigma}_{i M}^{(r)}\right\}^{-1} \mathbf{X}_{i}\right]^{-1}\left[\sum_{i=1}^{n} \frac{\nu+m_{i}}{\nu+u_{i M}^{(r)}} \mathbf{X}_{i}^{\top}\left\{\boldsymbol{\Sigma}_{i M}^{(r)}\right\}^{-1} \mathbf{Y}_{i}\right]
$$

em que o subíndice $M$ denota o método de estimação que estejamos considerando. Em seguida descreveremos os métodos de estimação para o modelo misto linear t-Student. 


\section{Estimação de máxima verossimilhança}

Considerando a função de verossimilhança dada na equação (4.16) temos que a função escore para o $j$-ésimo elemento do vetor $\boldsymbol{\theta}, \operatorname{com} j=1, \ldots, s$ fica dada por

$$
\begin{aligned}
\frac{\partial}{\partial \theta_{j}} l(\boldsymbol{\beta}, \boldsymbol{\theta} ; \mathbf{Y}) & =\frac{\partial}{\partial \theta_{j}}\left[-\frac{1}{2} \sum_{i=1}^{n} \log \left|\boldsymbol{\Sigma}_{i}\right|-\sum_{i=1}^{n} \frac{\nu+m_{i}}{2} \log \left(1+u_{i} / \nu\right)\right] \\
& =-\frac{1}{2} \sum_{i=1}^{n} \operatorname{tr}\left\{\boldsymbol{\Sigma}_{i}^{-1} \frac{\partial \boldsymbol{\Sigma}_{i}}{\partial \theta_{j}}\right\}+\frac{1}{2} \sum_{i=1}^{n} \frac{\nu+m_{i}}{\nu+u_{i}}\left[\frac{\partial u_{i}}{\partial \theta_{j}}\right]
\end{aligned}
$$

em que a derivada parcial de $u_{i}$ com respeito ao $j$-ésimo elemento do vetor $\boldsymbol{\theta}$ foi obtida na equação (4.13) para $j=1, \ldots, s$. Devemos especificar as estruturas para as matrizes $\boldsymbol{\Sigma}_{i}$ para obter as equações de estimação. Mas, em geral, não temos uma expressão explícita para os estimadores de $\boldsymbol{\theta}$.

A estimativa de $\boldsymbol{\beta}$ na $(\mathrm{r}+1)$-ésima iteração fica dada pela equação (4.17), em que o subíndice M é sustituido por MV. Então, a matriz $\boldsymbol{\Sigma}_{i M V}^{(r)}$ é a estimativa da matriz $\boldsymbol{\Sigma}_{i}$ obtida na $r$-ésima iteração pela maximização da equação (4.16) ou da solução de $\frac{\partial}{\partial \theta_{j}} l(\boldsymbol{\beta}, \boldsymbol{\theta} ; \mathbf{Y})=0$, para $j=1, \ldots, s$, considerando $\boldsymbol{\beta}=\boldsymbol{\beta}^{(r)}$.

\section{Estimação de máxima verossimilhança restrita}

Considerando os resultados obtidos na Seção 4.2 .2 e que $\mathbf{Y}_{i} \sim t_{m_{i}}\left(\mathbf{X}_{i} \boldsymbol{\beta}, \boldsymbol{\Sigma}_{i}, \nu\right)$, para $i=1, \ldots, n$, temos que a transformação fica dada por

$$
\begin{gathered}
\mathbf{W}_{i}^{\top} \mathbf{Y}_{i} \sim t_{m_{i}}\left(\mathbf{W}_{i}^{\top} \mathbf{X}_{i} \boldsymbol{\beta}, \mathbf{W}_{i}^{\top} \boldsymbol{\Sigma}_{i} \mathbf{W}_{i}, \nu\right) \\
{\left[\begin{array}{c}
\mathbf{W}_{i 1}^{\top} \mathbf{Y}_{i} \\
\mathbf{W}_{i 2}^{\top} \mathbf{Y}_{i}
\end{array}\right]=\left[\begin{array}{c}
\mathbf{V}_{i 1} \\
\mathbf{V}_{i 2}
\end{array}\right] \sim t_{m_{i}}\left\{\left[\begin{array}{c}
\boldsymbol{\beta} \\
\mathbf{0}
\end{array}\right],\left[\begin{array}{ll}
\mathbf{W}_{i 1}^{\top} \boldsymbol{\Sigma}_{i} \mathbf{W}_{i 1} & \mathbf{W}_{i 1}^{\top} \boldsymbol{\Sigma}_{i} \mathbf{W}_{i 2} \\
\mathbf{W}_{i 2}^{\top} \boldsymbol{\Sigma}_{i} \mathbf{W}_{i 1} & \mathbf{W}_{i 2}^{\top} \boldsymbol{\Sigma}_{i} \mathbf{W}_{i 2}
\end{array}\right], \nu\right\} .}
\end{gathered}
$$

Portanto, o logaritmo da função de verossimilhança marginal para as transformações dos $\mathbf{V}_{i 2}$ fica dado por

$$
\sum_{i=1}^{n} l\left(\boldsymbol{\theta} ; \mathbf{V}_{i 2}\right)=c t e-\frac{1}{2} \sum_{i=1}^{n} \log \left|\boldsymbol{\Sigma}_{i}\right|-\frac{1}{2} \sum_{i=1}^{n} \log \left|\mathbf{X}_{i}^{\top} \boldsymbol{\Sigma}_{i}^{-1} \mathbf{X}_{i}\right|-\sum_{i=1}^{n} \frac{\nu+m_{i}-p}{2} \log \left(1+u_{i 2} / \nu\right),
$$

sendo que $u_{i 2}=\left(\mathbf{Y}_{i}-\mathbf{X}_{i} \mathbf{b}_{i}\right)^{\top} \boldsymbol{\Sigma}_{i}^{-1}\left(\mathbf{Y}_{i}-\mathbf{X}_{i} \mathbf{b}_{i}\right)$ e $\mathbf{b}_{i}=\left(\mathbf{X}_{i}^{\top} \boldsymbol{\Sigma}_{i}^{-1} \mathbf{X}_{i}\right)^{-1} \mathbf{X}_{i}^{\top} \boldsymbol{\Sigma}_{i}^{-1} \mathbf{Y}_{i}$. A equação de estimação com respeito de $\theta_{j}$ fica dada por

$$
\begin{aligned}
-\frac{1}{2} \sum_{i=1}^{n} \operatorname{tr}\left\{\boldsymbol{\Sigma}_{i}^{-1} \frac{\partial \boldsymbol{\Sigma}_{i}}{\partial \theta_{j}}\right\} & -\frac{1}{2} \sum_{i=1}^{n} \operatorname{tr}\left\{\left(\mathbf{X}_{i}^{\top} \boldsymbol{\Sigma}_{i}^{-1} \mathbf{X}_{i}\right)^{-1} \mathbf{X}_{i}^{\top} \boldsymbol{\Sigma}_{i}^{-1} \frac{\partial \boldsymbol{\Sigma}_{i}}{\partial \theta_{j}} \boldsymbol{\Sigma}_{i}^{-1} \mathbf{X}_{i}\right\} \\
& +\frac{1}{2 \nu} \sum_{i=1}^{n}\left(\nu+m_{i}-p\right)\left(1+u_{i 2} / \nu\right)^{-1}\left[\frac{\partial u_{i 2}}{\partial \theta_{j}}\right]=0
\end{aligned}
$$

e a derivada parcial de $u_{i 2}$ com respeito a $\theta_{j}$ foi obtida na equação (4.11), para $i=1, \ldots, n$ e $j=1, \ldots, s$. Neste caso, a equação para estimar o vetor $\boldsymbol{\beta}$ pode ser expressa pelo processo iterativo

$$
\boldsymbol{\beta}_{M V R}^{(r+1)}=\left\{\sum_{i=1}^{n} \frac{\nu+m_{i}}{\nu+u_{i}^{(r)}} \mathbf{X}_{i}^{\top} \widehat{\boldsymbol{\Sigma}}_{i M V R}^{-1} \mathbf{X}_{i}\right\}^{-1}\left\{\sum_{i=1}^{n} \frac{\nu+m_{i}}{\nu+u_{i}^{(r)}} \mathbf{X}_{i}^{\top} \widehat{\boldsymbol{\Sigma}}_{i M V R}^{-1} \mathbf{Y}_{i}\right\},
$$


para $r=0,1, \ldots$, em que $u_{i}^{(r)}=\left(\mathbf{Y}_{i}-\mathbf{X}_{i} \boldsymbol{\beta}_{i M V R}^{(r)}\right)^{\top} \widehat{\boldsymbol{\Sigma}}_{i M V R}^{-1}\left(\mathbf{Y}_{i}-\mathbf{X}_{i} \boldsymbol{\beta}_{i M V R}^{(r)}\right)$ para $i=1, \ldots, n$. A matriz $\widehat{\boldsymbol{\Sigma}}_{i M V R}$ é a estimativa da matriz $\boldsymbol{\Sigma}_{i}$ obtida da maximização da equação (4.18) ou da solução da equação (4.19), para $j=1, \ldots, s$.

\section{Estimação baseada na função de verossimilhança perfilada modificada}

Considerando os resultados obtidos na Seção 3.4 temos que $l_{p}(\boldsymbol{\theta})$ para o modelo misto linear t-Student fica dado por

$$
l_{p}(\boldsymbol{\theta})=c t e-\frac{1}{2} \sum_{i=1}^{n} \log \left|\boldsymbol{\Sigma}_{i}\right|-\sum_{i=1}^{n} \frac{\nu+m_{i}}{2} \log \left\{1+\hat{u}_{i \theta} / \nu\right\},
$$

em que $\hat{u}_{i \theta}=\left(\mathbf{Y}_{i}-\mathbf{X}_{i} \hat{\boldsymbol{\beta}}_{\theta}\right)^{\top} \boldsymbol{\Sigma}_{i}^{-1}\left(\mathbf{Y}_{i}-\mathbf{X}_{i} \hat{\boldsymbol{\beta}}_{\theta}\right)$ para $i=1, \ldots, n$.

\section{Ajuste da função de verossimilhança perfilada modificada}

A matriz de informação de $\boldsymbol{\beta}$ avaliada no estimador de máxima verossimilhança de $\boldsymbol{\beta}$, dada na equação (4.12) para o modelo misto linear elíptico, fica dada por

$$
\mathbf{J}_{\beta \beta}\left(\hat{\boldsymbol{\beta}}_{\theta}\right)=4 \sum_{i=1}^{n}\left\{\left(\nu+m_{i}\right) / 2 \nu^{2}\right\}\left(1+\hat{u}_{i \theta} / \nu\right)^{-2} \mathbf{X}_{i}^{\top} \boldsymbol{\Sigma}_{i}^{-1} \mathbf{X}_{i}
$$

Assim, temos que a função de verossimilhança perfilada modificada de $\boldsymbol{\theta}$ neste modelo assume a forma

$$
\begin{aligned}
l_{p m}(\boldsymbol{\theta})= & -\frac{1}{2} \sum_{i=1}^{n} \log \left|\boldsymbol{\Sigma}_{i}\right|-\frac{1}{2} \sum_{i=1}^{n}\left(\nu+m_{i}\right) \log \left\{1+\hat{u}_{i \theta} / \nu\right\} \\
& -\frac{1}{2} \log \left|4 \sum_{i=1}^{n} \frac{\nu+m_{i}}{2 \nu^{2}}\left(1+\hat{u}_{i \theta} / \nu\right)^{-2} \mathbf{X}_{i}^{\top} \boldsymbol{\Sigma}_{i}^{-1} \mathbf{X}_{i}\right|
\end{aligned}
$$

A equação de estimação para $\theta_{j}$ resultante fica dada por

$$
\begin{array}{r}
\frac{1}{4} \operatorname{tr}\left[\left\{\sum_{i=1}^{n} \frac{\nu+m_{i}}{\nu}\left(1+\hat{u}_{i \theta} / \nu\right)^{-2} \mathbf{X}_{i}^{\top} \boldsymbol{\Sigma}_{i}^{-1} \mathbf{X}_{i}\right\}^{-1} \sum_{i=1}^{n} \frac{\nu+m_{i}}{\nu^{3}}\left(1+\hat{u}_{i \theta} / \nu\right)^{-2}\right. \\
\left.\left\{\left(1+\hat{u}_{i \theta} / \nu\right)^{-1}\left[\frac{\partial \hat{u}_{i \theta}}{\partial \theta_{j}}\right] \mathbf{X}_{i}^{\top} \boldsymbol{\Sigma}_{i}^{-1} \mathbf{X}_{i}+\mathbf{X}_{i}^{\top} \boldsymbol{\Sigma}_{i}^{-1} \frac{\partial \boldsymbol{\Sigma}_{i}}{\partial \theta_{j}} \boldsymbol{\Sigma}_{i}^{-1} \mathbf{X}_{i}\right\}\right] \\
-\frac{1}{2} \sum_{i=1}^{n} \operatorname{tr}\left\{\boldsymbol{\Sigma}_{i}^{-1} \frac{\partial \boldsymbol{\Sigma}_{i}}{\partial \theta_{j}}\right\}-\frac{1}{2} \sum_{i=1}^{n} \frac{\nu+m_{i}}{\nu}\left(1+\hat{u}_{i \theta} / \nu\right)^{-1}\left[\frac{\partial \hat{u}_{i \theta}}{\partial \theta_{j}}\right]=0
\end{array}
$$

em que a derivada parcial de $\hat{u}_{i \theta}$ com respeito de $\theta_{j}$ foi expresa na equação (4.13), para $j=1, \ldots, s$ e $i=1, \ldots, n$.

\section{Ajuste da função de verossimilhança perfilada modificada aproximada}

Uma aproximação da função de verossimilhança perfilada modificada pode ser considerada utilizando a matriz de informação esperada ao invés da matriz de informação de $\boldsymbol{\beta}$. A matriz de 
informação esperada avaliada no estimador de $\boldsymbol{\beta}$ fica dada por

$$
\mathbf{K}_{\beta \beta}\left(\hat{\boldsymbol{\beta}}_{\theta}\right)=\sum_{i=1}^{n} \frac{\nu+m_{i}}{\nu+m_{i}+2} \mathbf{X}_{i}^{\top} \boldsymbol{\Sigma}_{i}^{-1} \mathbf{X}_{i}
$$

Vemos que esta última expressão não depende da estimativa $\hat{\boldsymbol{\beta}}_{\theta}$ e a aproximação da função de verossimilhança perfilada modificada fica dada por

$$
l_{p m a}(\boldsymbol{\theta})=-\frac{1}{2} \sum_{i=1}^{n} \log \left|\boldsymbol{\Sigma}_{i}\right|-\frac{1}{2} \sum_{i=1}^{n}\left(\nu+m_{i}\right) \log \left\{1+\hat{u}_{i \theta} / \nu\right\}-\frac{1}{2} \log \left|\sum_{i=1}^{n} \frac{\nu^{2}\left(\nu+m_{i}\right)}{\nu+m_{i}+2} \mathbf{X}_{i}^{\top} \boldsymbol{\Sigma}_{i}^{-1} \mathbf{X}_{i}\right| .
$$

A equação de estimação assume a forma

$$
\begin{gathered}
\frac{1}{4} \operatorname{tr}\left[\left\{\sum_{i=1}^{n} \frac{\nu^{2}\left(\nu+m_{i}\right)}{\nu+m_{i}+2} \mathbf{X}_{i}^{\top} \boldsymbol{\Sigma}_{i}^{-1} \mathbf{X}_{i}\right\}^{-1} \sum_{i=1}^{n} \frac{\nu^{2}\left(\nu+m_{i}\right)}{\nu+m_{i}+2} \mathbf{X}_{i}^{\top} \boldsymbol{\Sigma}_{i}^{-1} \frac{\partial \boldsymbol{\Sigma}_{i}}{\partial \theta_{j}} \boldsymbol{\Sigma}_{i}^{-1} \mathbf{X}_{i}\right] \\
-\frac{1}{2} \sum_{i=1}^{n} \operatorname{tr}\left\{\boldsymbol{\Sigma}_{i}^{-1} \frac{\partial \boldsymbol{\Sigma}_{i}}{\partial \theta_{j}}\right\}-\frac{1}{2} \sum_{i=1}^{n}\left(\nu+m_{i}\right)\left(\nu+\hat{u}_{i \theta}\right)^{-1}\left[\frac{\partial \hat{u}_{i \theta}}{\partial \theta_{j}}\right]=0,
\end{gathered}
$$

em que a derivada parcial de $\hat{u}_{i \theta}$ com respeito de $\theta_{j}$ foi dada na equação $(4.13)$, para $j=1, \ldots, s$ e $i=1, \ldots, n$. Nestes últimos métodos de estimação a estimativa de $\boldsymbol{\beta}$ é obtida de forma similar ao caso da estimativa de máxima verossimilhança restrita.

\subsubsection{Modelo misto linear exponencial potência}

Consideraremos o modelo apresentado na equação (4.1) e que o vetor $\left(\mathbf{Y}_{i}^{\top}, \mathbf{d}_{i}^{\top}\right)^{\top}$ tem distribuição elíptica na forma dada na equação $(4.2)$, para $i=1, \ldots, n$, com função geradora de densidade $g^{*}(\cdot)$ que não precisamos especificar mas sim supor que existe. Assim, para definir o modelo misto linear exponencial potência vamos supor que a amostra aleatória $\mathbf{Y}_{1}, \ldots, \mathbf{Y}_{n}$, em que os vetores $\mathbf{Y}_{i}$ são independentes com distribuição marginal exponencial potência dada por

$$
\mathbf{Y}_{i} \sim E P_{m_{i}}\left(\boldsymbol{\mu}_{i}, \boldsymbol{\Sigma}_{i}, \lambda\right), \text { para } i=1, \ldots, n,
$$

em que $\boldsymbol{\Sigma}_{i}=\mathbf{Z}_{i} \mathbf{D}_{i} \mathbf{Z}_{i}^{\top}+\boldsymbol{\Sigma}_{m_{i}}$ e $\boldsymbol{\theta}=\left(\boldsymbol{\gamma}^{\top}, \boldsymbol{\alpha}^{\top}\right)^{\top}$ é um vetor de dimensão $s$, que contém os parâmetros desconhecidos de todas as matrizes $\boldsymbol{\Sigma}_{i}$, com $i=1, \ldots, n$. Para cada $\mathbf{Y}_{i}$ consideramos as especificações da distribuição exponencial potência dadas na Seção 3.6.2. Assim, a função de verossimilhança para este modelo fica dada por

$$
L(\boldsymbol{\beta}, \boldsymbol{\theta} ; \mathbf{Y})=\prod_{i=1}^{n} L\left(\boldsymbol{\beta}, \boldsymbol{\theta} ; \mathbf{Y}_{i}\right)=\prod_{i=1}^{n}\left|\boldsymbol{\Sigma}_{i}\right|^{-1 / 2} c_{3 i} \exp \left(-\frac{u_{i}^{\lambda}}{2}\right)
$$

em que $u_{i}$ é a distância dada por $\left(\mathbf{Y}_{i}-\mathbf{X}_{i} \boldsymbol{\beta}\right)^{\top} \boldsymbol{\Sigma}_{i}^{-1}\left(\mathbf{Y}_{i}-\mathbf{X}_{i} \boldsymbol{\beta}\right)$, o parâmetro $\lambda$ será assumido conhecido e portanto o termo $c_{3 i}=m_{i} k\left(m_{i}-\frac{m_{i}+2}{\lambda}, \frac{m_{i}+2}{\lambda}\right) /\left(2^{\frac{m_{i}+2}{\lambda}}\right)$ não depende de parâmetros desconhecidos. A função $k(\cdot)$ foi definida na equação (3.4) da Seção 3.1.

O logaritmo da função de verossimilhança fica expressa na forma

$$
\begin{aligned}
l(\boldsymbol{\beta}, \boldsymbol{\theta} ; \mathbf{Y})=\sum_{i=1}^{n} l\left(\boldsymbol{\beta}, \boldsymbol{\theta} ; \mathbf{Y}_{i}\right) & =-\frac{1}{2} \sum_{i=1}^{n} \log \left|\boldsymbol{\Sigma}_{i}\right|+\sum_{i=1}^{n} \log c_{3 i}-\frac{1}{2} \sum_{i=1}^{n} u_{i}^{\lambda} \\
& =c t e-\frac{1}{2} \sum_{i=1}^{n} \log \left|\boldsymbol{\Sigma}_{i}\right|-\frac{1}{2} \sum_{i=1}^{n} u_{i}^{\lambda}
\end{aligned}
$$


Do mesmo modo que o modelo misto linear t-Student, podemos escrever a fórmula geral das equações de estimação de $\boldsymbol{\beta}$ conforme o processo iterativo

$$
\boldsymbol{\beta}_{M V}^{(r+1)}=\left[\sum_{i=1}^{n} u_{i}^{(r)}{ }^{\lambda-1} \mathbf{X}_{i}^{\top} \boldsymbol{\Sigma}_{i M V}^{(r)}{ }^{-1} \mathbf{X}_{i}\right]^{-1}\left[\sum_{i=1}^{n} u_{i}^{(r){ }^{\lambda-1}} \mathbf{X}_{i}^{\top} \boldsymbol{\Sigma}_{i M V}^{(r)}{ }^{-1} \mathbf{Y}_{i}\right],
$$

em que o subíndice $M$ denota o método de estimação que estamos considerando. Em seguida descreveremos os métodos de estimação para o modelo misto linear exponencial potência.

\section{Estimação de máxima verossimilhança}

A função escore para o $j$-ésimo elemento do vetor $\boldsymbol{\theta}$, com $j=1, \ldots, s$, fica dada por

$$
\begin{aligned}
\frac{\partial}{\partial \theta_{j}} l(\boldsymbol{\beta}, \boldsymbol{\theta} ; \mathbf{Y}) & =\frac{\partial}{\partial \theta_{j}}\left[-\frac{1}{2} \sum_{i=1}^{n} \log \left|\boldsymbol{\Sigma}_{i}\right|-\frac{1}{2} \sum_{i=1}^{n} u_{i}^{\lambda}\right] \\
& =-\frac{1}{2} \sum_{i=1}^{n} \operatorname{tr}\left\{\boldsymbol{\Sigma}_{i}^{-1} \frac{\partial \boldsymbol{\Sigma}_{i}}{\partial \theta_{j}}\right\}-\frac{\lambda}{2} \sum_{i=1}^{n} u_{i}^{\lambda-1}\left[\frac{\partial u_{i}}{\partial \theta_{j}}\right]
\end{aligned}
$$

em que a derivada parcial de $u_{i}$ com respeito ao $j$-ésimo elemento do vetor $\boldsymbol{\theta}$ já foi obtida na equação (4.6) para $j=1, \ldots, s$ e $i=1, \ldots, n$. Por último, considerando algum método de otimização para determinar as estimativas de máxima verossimilhança para o vetor $\boldsymbol{\beta}$ da equação (4.22), em que o subíndice M é sustituido por MV. A matriz $\boldsymbol{\Sigma}_{i M V}^{(r)}$ é a estimativa da matriz $\boldsymbol{\Sigma}_{i}$ obtida na $r$-ésima iteração da maximização da equação (4.16) ou da solução de $\frac{\partial}{\partial \theta_{j}} l(\boldsymbol{\beta}, \boldsymbol{\theta} ; \mathbf{Y})=0$, para $j=1, \ldots, s$, considerando $\boldsymbol{\beta}=\boldsymbol{\beta}^{(r)}$.

\section{Estimação de máxima verossimilhança restrita}

Considerando os resultados da estimação de máxima verossimilhança restrita para o modelo misto linear elíptico obtidos na Seção 4.2.2 e da transformação para o modelo linear exponencial potência visto na Seção 3.6.2, temos que a $i$-ésima transformação $\mathbf{W}_{i 2}^{\top} \mathbf{Y}_{i 2}$ fica dada por

$$
\mathbf{W}_{i 2}^{\top} \mathbf{Y}_{i}=\mathbf{V}_{i 2} \sim E l_{m_{i}-p}\left(\mathbf{0}, \mathbf{W}_{i 2}^{\top} \boldsymbol{\Sigma}_{i} \mathbf{W}_{i 2}, g_{2}\right), \text { para } i=1, \ldots, n
$$

em que $g_{2}(\cdot)$ é a função geradora de densidade associada à transformação $\mathbf{W}_{i 2}^{\top} \mathbf{Y}_{i}$ que fica expressa na mesma forma que foi especificada para o modelo linear exponencial potência na equação (3.33).

Assim, o logaritmo da função de verossimilhança marginal para as transformações dos $\mathbf{V}_{i 2}$ fica dado por

$$
\sum_{i=1}^{n} l\left(\boldsymbol{\theta} ; \mathbf{V}_{i 2}\right)=c t e-\frac{1}{2} \sum_{i=1}^{n} \log \left|\boldsymbol{\Sigma}_{i}\right|-\frac{1}{2} \sum_{i=1}^{n} \log \left|\mathbf{X}_{i}^{\top} \boldsymbol{\Sigma}_{i}^{-1} \mathbf{X}_{i}\right|-\sum_{i=1}^{n} \log g\left(u_{i 2}\right),
$$

sendo que $u_{i 2}=\left(\mathbf{Y}_{i}-\mathbf{X}_{i} \mathbf{b}_{i}\right)^{\top} \boldsymbol{\Sigma}_{i}^{-1}\left(\mathbf{Y}_{i}-\mathbf{X}_{i} \mathbf{b}_{i}\right)$ e $\mathbf{b}_{i}=\left(\mathbf{X}_{i}^{\top} \boldsymbol{\Sigma}_{i}^{-1} \mathbf{X}_{i}\right)^{-1} \mathbf{X}_{i}^{\top} \boldsymbol{\Sigma}_{i}^{-1} \mathbf{Y}_{i}$. A equação de estimação com respeito de $\theta_{j}$ de forma geral foi dada na equação (4.10). Neste caso a função $w_{2}\left(u_{i 2}\right)$ assume a forma

$$
w_{2}\left(u_{i 2}\right)=\frac{m-p}{2 u_{i 2}}+\frac{d \int_{0}^{1} w^{\frac{p-m_{i}}{2}-1} \exp \left\{-\frac{1}{2}\left(\frac{u_{i 2}}{w}\right)^{\lambda}\right\} d w}{\int_{0}^{1} w^{\frac{p-m_{i}}{2}-1} \exp \left\{-\frac{1}{2}\left(\frac{u_{i 2}}{w}\right)^{\lambda}\right\} d w} .
$$

A estimativa de máxima verossimilhança restrita do vetor $\boldsymbol{\beta}$ é obtida da solução da equação (4.22), substituindo o subíndice M por MVR. A matriz $\widehat{\boldsymbol{\Sigma}}_{i M V R}$ é a estimativa da matriz $\boldsymbol{\Sigma}_{i}$ obtida da maximização da equação (4.23) com respeito ao vetor $\boldsymbol{\theta}$ ou da solução de um sistema de equações. 
O termo $u_{i}^{(r)}=\left(\mathbf{Y}_{i}-\mathbf{X}_{i} \boldsymbol{\beta}_{i M V R}^{(r)}\right)^{\top} \widehat{\boldsymbol{\Sigma}}_{i M V R}^{-1}\left(\mathbf{Y}_{i}-\mathbf{X}_{i} \boldsymbol{\beta}_{i M V R}^{(r)}\right)$, para $i=1, \ldots, n$.

\section{Estimação baseada na função de verossimilhança perfilada modificada}

Considerando os resultados obtidos na Seção 3.4 temos que $l_{p}(\boldsymbol{\theta})$ para o modelo misto linear exponencial potência fica dado por

$$
l_{p}(\boldsymbol{\theta})=\text { cte }-\frac{1}{2} \sum_{i=1}^{n} \log \left|\boldsymbol{\Sigma}_{i}\right|-\frac{1}{2} \sum_{i=1}^{n} \hat{u}_{i \theta}^{\lambda},
$$

em que $\hat{u}_{i \theta}=\left(\mathbf{Y}_{i}-\mathbf{X}_{i} \hat{\boldsymbol{\beta}}_{\theta}\right)^{\top} \boldsymbol{\Sigma}_{i}^{-1}\left(\mathbf{Y}_{i}-\mathbf{X}_{i} \hat{\boldsymbol{\beta}}_{\theta}\right)$, para $i=1, \ldots, n$.

\section{Ajuste da função de verossimilhança perfilada modificada}

Na equação (4.12) apresentamos a matriz de informação de $\boldsymbol{\beta}$ avaliada no estimador de máxima verossimilhança de $\boldsymbol{\beta}$ para o modelo misto linear elíptico. Entanto que $w_{g}(\cdot)$ para a distribuição exponencial potência foi dada na Tabela 3.2. Assim, temos que a matriz de informação com respeito a $\boldsymbol{\beta}$ avaliada em $\hat{\boldsymbol{\beta}}_{\theta}$ fica dada por

$$
\mathbf{J}_{\beta \beta}\left(\hat{\boldsymbol{\beta}}_{\theta}\right)=-2 \sum_{i=1}^{n} \lambda \hat{u}_{i \theta}^{\lambda-1} \mathbf{X}_{i}^{\top} \boldsymbol{\Sigma}_{i}^{-1} \mathbf{X}_{i} .
$$

A função de verossimilhança perfilada modificada de $\boldsymbol{\theta}$ neste modelo fica dada por

$$
l_{p m}(\boldsymbol{\theta})=-\frac{1}{2} \sum_{i=1}^{n} \log \left|\boldsymbol{\Sigma}_{i}\right|-\frac{1}{2} \sum_{i=1}^{n} \hat{u}_{i \theta}^{\lambda}-\frac{1}{2} \log \left|-2 \sum_{i=1}^{n} \lambda \hat{u}_{i \theta}^{\lambda-1} \mathbf{X}_{i}^{\top} \boldsymbol{\Sigma}_{i}^{-1} \mathbf{X}_{i}\right| .
$$

A equação de estimação para $\theta_{j}$ resultante assume a forma

$$
\begin{gathered}
\frac{1}{4} \operatorname{tr}\left[\left\{\sum_{i=1}^{n} \lambda \hat{u}_{i \theta}^{\lambda-1} \mathbf{X}_{i}^{\top} \boldsymbol{\Sigma}_{i}^{-1} \mathbf{X}_{i}\right\}^{-1} \sum_{i=1}^{n} \lambda \hat{u}_{i \theta}^{\lambda-2}\left\{(\lambda-1)\left[\frac{\partial \hat{u}_{i \theta}}{\partial \theta_{j}}\right] \mathbf{X}_{i}^{\top} \boldsymbol{\Sigma}_{i}^{-1} \mathbf{X}_{i}-\hat{u}_{i \theta} \mathbf{X}_{i}^{\top} \boldsymbol{\Sigma}_{i}^{-1} \frac{\partial \boldsymbol{\Sigma}_{i}}{\partial \theta_{j}} \boldsymbol{\Sigma}_{i}^{-1} \mathbf{X}_{i}\right\}\right] \\
-\frac{1}{2} \sum_{i=1}^{n} \operatorname{tr}\left\{\boldsymbol{\Sigma}_{i}^{-1} \frac{\partial \boldsymbol{\Sigma}_{i}}{\partial \theta_{j}}\right\}-\frac{\lambda}{2} \sum_{i=1}^{n} \hat{u}_{i \theta}^{\lambda-1}\left[\frac{\partial \hat{u}_{i \theta}}{\partial \theta_{j}}\right]=0,
\end{gathered}
$$

em que a derivada parcial de $\hat{u}_{i \theta}$ com respeito de $\theta_{j}$ foi obtida na equação (4.13), para $j=1, \ldots, s$ e $i=1, \ldots, n$.

\section{Ajuste da função de verossimilhança perfilada modificada aproximada}

A matriz de informação esperada com respeito a $\boldsymbol{\beta}$ fica dada por

$$
\mathbf{K}_{\beta \beta}\left(\hat{\boldsymbol{\beta}}_{\theta}\right)=\sum_{i=1}^{n} \frac{4 \lambda^{2}}{2^{1 / \lambda}} \frac{\Gamma\left(\frac{m_{i}-2}{2 \lambda}+2\right)}{m_{i} \Gamma\left(\frac{m_{i}}{2 \lambda}\right)} \mathbf{X}_{i}^{\top} \boldsymbol{\Sigma}_{i}^{-1} \mathbf{X}_{i}
$$

Denotaremos por $C_{m i}$ o fator $\frac{4 \lambda^{2}}{2^{1 / \lambda}} \frac{\Gamma\left(\frac{m_{i}-2}{2 \lambda}+2\right)}{m_{i} \Gamma\left(\frac{m_{i}}{2 \lambda}\right)}$ que não depende dos parâmetros desconhecidos, então a matriz de informação esperada fica dada por

$$
\mathbf{K}_{\beta \beta}\left(\hat{\boldsymbol{\beta}}_{\theta}\right)=\sum_{i=1}^{n} C_{m i} \mathbf{X}_{i}^{\top} \boldsymbol{\Sigma}_{i}^{-1} \mathbf{X}_{i}
$$


Vemos que esta última expressão não depende da estimativa $\hat{\boldsymbol{\beta}}_{\theta}$ e a aproximação da função de verossimilhança perfilada modificada assume a forma

$$
l_{p m a}(\boldsymbol{\theta})=-\frac{1}{2} \sum_{i=1}^{n} \log \left|\boldsymbol{\Sigma}_{i}\right|-\frac{1}{2} \sum_{i=1}^{n} \hat{u}_{i \theta}^{\lambda}-\frac{1}{2} \log \left|\sum_{i=1}^{n} C_{m i} \mathbf{X}_{i}^{\top} \boldsymbol{\Sigma}_{i}^{-1} \mathbf{X}_{i}\right| .
$$

A equação de estimação fica dada por

$$
\begin{gathered}
\frac{1}{4} \operatorname{tr}\left[\left\{\sum_{i=1}^{n} C_{m i} \mathbf{X}_{i}^{\top} \boldsymbol{\Sigma}_{i}^{-1} \mathbf{X}_{i}\right\}^{-1} \sum_{i=1}^{n}\left\{C_{m i} \mathbf{X}_{i}^{\top} \boldsymbol{\Sigma}_{i}^{-1} \frac{\partial \boldsymbol{\Sigma}_{i}}{\partial \theta_{j}} \boldsymbol{\Sigma}_{i}^{-1} \mathbf{X}_{i}\right\}\right] \\
-\frac{1}{2} \sum_{i=1}^{n} \operatorname{tr}\left\{\boldsymbol{\Sigma}_{i}^{-1} \frac{\partial \boldsymbol{\Sigma}_{i}}{\partial \theta_{j}}\right\}-\frac{\lambda}{2} \sum_{i=1}^{n} \hat{u}_{i \theta}^{\lambda-1}\left[\frac{\partial \hat{u}_{i \theta}}{\partial \theta_{j}}\right]=0,
\end{gathered}
$$

em que a derivada parcial de $\hat{u}_{i \theta}$ com respeito de $\theta_{j}$ foi dada na equação (4.13), para $j=1, \ldots, s$ e $i=1, \ldots, n$. Nestes últimos métodos de estimação a estimativa de $\boldsymbol{\beta}$ é obtida resolvendo as equações de máxima verossimilhança restrita.

\subsection{Comentários}

Neste capítulo estendemos os métodos de estimação apresentados num modelo linear elíptico no Capítulo 3 para um modelo misto linear elíptico. Segundo a formulação do modelo (4.2), em geral, a estimativa de máxima verossimilhança de $\boldsymbol{\beta}$ não tem forma explícita e a equação de estimação para este vetor de parâmetros depende da função geradora de densidade $g(\cdot)$ através de $w_{g}(\cdot)$. Portanto, o processo de estimação de máxima verossimilhança é mais complexo do que no modelo linear elíptico e, por conseguinte na formulação dos outros métodos de estimação que dependem da função de verossimilhança considerados neste trabalho. Para determinar os estimadores da matriz $\boldsymbol{\Sigma}(\boldsymbol{\theta})$, precisamos especificar sua estrutura, mas dada a forma das equações de estimação, especificadas para cada método de estimação na Seção 4.2, usualmente os estimadores não terão formas fechadas.

No método de estimação restrita, Seção 4.2.2, utilizamos para cada vetor de respostas, $\mathbf{Y}_{i}$, a transformação proposta por Verbyla (1990). Desta proposta de transformação resulta uma substituição de $\boldsymbol{\beta}$ por uma correção local dada por $\mathbf{b}_{i}=\left(\mathbf{X}_{i}^{\top} \boldsymbol{\Sigma}_{i}^{-1} \mathbf{X}_{i}\right)^{-1} \mathbf{X}_{i}^{\top} \boldsymbol{\Sigma}_{i}^{-1} \mathbf{Y}_{i}$ no logaritmo da função de verossimilhança marginal dos parâmetros $\boldsymbol{\theta}$, para $i=1, \ldots, n$. Esta formulação permite que $\mathbf{V}_{i}=\mathbf{W}_{i} \mathbf{Y}_{i}$ tenha uma distribuição elíptica e podemos utilizar as propriedades e resultados obtidos para o modelo linear elíptico (Capítulo 3). Mas esta transformação não permite recuperar o caso usual de MVR para o modelo misto linear normal em que a variável $u_{i 2}$ fica dada por $\left(\mathbf{Y}_{i}-\mathbf{X}_{i} \hat{\boldsymbol{\beta}}\right)^{\top} \boldsymbol{\Sigma}_{i}^{-1}\left(\mathbf{Y}_{i}-\mathbf{X}_{i} \hat{\boldsymbol{\beta}}\right)$, sendo $\hat{\boldsymbol{\beta}}$ a estrutura do estimador de máxima verossimilhança de $\boldsymbol{\beta}$ dado na equação (4.15).

Nos casos particulares dos modelos misto linear t-Student e misto linear exponencial potência, vimos que não temos expressões fechadas para os estimadores, mesmo quando especificamos uma estrutura para a matriz $\boldsymbol{\Sigma}$. Contudo, temos uma expressão fechada para os estimadores de $\boldsymbol{\beta}$. Da mesma forma que o modelo linear exponencial potência, vimos neste capítulo que a proposta da verossimilhança restrita para o vetor $\boldsymbol{\theta}$ fica dependendo da função de verossimilhança marginal de $\mathbf{V}_{2}$.

No entanto, para o modelo misto linear normal os ajustes da função de verossimilhança perfilada levam aos mesmos resultados uma vez que as matrizes de informação observada e esperada coincidem.

Para comparar as estimativas pelos distintos métodos de estimação, no Capítulo 6 apresentamos 
simulações de um modelo misto linear t-Student. Mediante um procedimento bootstrap não paramétrico obtivemos uma aproximação dos errores padrão das estimativas. De forma similar como foi comentado na Seção 3.7 do Capítulo 3 pode ser interessante aplicar o ajuste das funções escore da verossimilhança perfilada proposto por McCullagh e Tibshirani (1990) nos modelos mistos lineares elípticos.

No próximo capítulo discutimos a extensão de uma aproximação alternativa para a função de verossimilhança restrita de um modelo t-heteroscedástico baseado no método de aproximação parcial de Laplace proposto por Taylor e Verbyla (2006), para um modelo misto linear t-Student. 


\section{Capítulo 5}

\section{Procedimento alternativo de estimação restrita}

Discutiremos neste capítulo uma extensão do procedimento de estimação restrita proposto por Taylor e Verbyla (2006) em modelos lineares heteroscedásticos com erros t-Student, para o modelo misto linear t-Student. Para que a metodologia seja aplicada, iremos considerar a forma de mistura de escala normal discutida em Pinheiro et al. (2001) para o modelo mistos linear t-Student.

\subsection{Formulação da função de verossimilhança restrita}

Vamos supor que a distribuição conjunta $\left(\mathbf{Y}_{i}^{\top}, \mathbf{d}_{i}^{\top}\right)^{\top}$ é t-Student com $\nu$ graus de liberdade, tal que

$$
\left[\begin{array}{c}
\mathbf{Y}_{i} \\
\mathbf{d}_{i}
\end{array}\right] \sim t_{m_{i}+q}\left(\left[\begin{array}{c}
\mathbf{X}_{i} \boldsymbol{\beta} \\
\mathbf{0}
\end{array}\right],\left[\begin{array}{cc}
\mathbf{Z}_{i} \mathbf{D} \mathbf{Z}_{i}^{\top}+\mathbf{\Sigma}_{m_{i}} & \mathbf{Z}_{i} \mathbf{D} \\
\mathbf{D Z}_{i}^{\top} & \mathbf{D}
\end{array}\right], \nu\right)
$$

Então, a distribuição marginal $\mathbf{Y}_{i} \sim t_{m_{i}}\left(\mathbf{X}_{i} \boldsymbol{\beta}, \boldsymbol{\Sigma}_{i}(\boldsymbol{\theta}), \nu\right)$, em que $\boldsymbol{\Sigma}_{i}(\boldsymbol{\theta})=\mathbf{Z}_{i} \mathbf{D} \mathbf{Z}_{i}^{\top}+\boldsymbol{\Sigma}_{m_{i}}$ e $\boldsymbol{\theta}=$ $\left(\boldsymbol{\alpha}^{\top}, \boldsymbol{\gamma}^{\top}\right)^{\top}$ é o vetor de dimensão $s$, que contém todos os parâmetros desconhecidos de todas as matrizes $\boldsymbol{\Sigma}_{i}(\boldsymbol{\theta})$, com $i=1, \ldots, n$.

Considerando a formulação para um modelo linear de efeitos mistos t-Student proposta por Pinheiro et al. (2001)(equação (3.7), pág.6) podemos escrever a distribuição marginal de $\mathbf{Y}_{i}$ como o seguinte modelo hierárquico:

$$
\mathbf{Y}_{i} \mid \omega_{i} \sim N_{m i}\left(\mathbf{X}_{i} \boldsymbol{\beta},\left(1 / \omega_{i}\right)\left(\mathbf{Z}_{i} \mathbf{D} \mathbf{Z}_{i}^{\top}+\boldsymbol{\Sigma}_{m_{i}}\right)\right), \quad \omega_{i} \sim \operatorname{Gamma}(\nu / 2, \nu / 2)
$$

em que $\omega_{i}, i=1, \ldots, n$, pode ser considerado como dados faltantes e consideramos a matriz $\boldsymbol{\Sigma}_{i}=$ $\left(1 / \omega_{i}\right) \boldsymbol{\Sigma}_{i}(\boldsymbol{\theta})=\left(1 / \omega_{i}\right)\left(\mathbf{Z}_{i} \mathbf{D} \mathbf{Z}_{i}^{\top}+\boldsymbol{\Sigma}_{m_{i}}\right)$. No Apêndice E explicamos brevemente esta metodologia proposta por Taylor e Verbyla (2006). O conjunto de parâmetros como $\boldsymbol{\lambda}=\left(\boldsymbol{\beta}^{\top}, \boldsymbol{\eta}^{\top}\right)$ com $\boldsymbol{\eta}=$ $\left(\boldsymbol{\theta}^{\top}, \nu\right)^{\top}$. Portanto, a função de verossimilhança fica dada por

$$
L(\boldsymbol{\beta}, \boldsymbol{\eta} ; \mathbf{Y})=\int_{\mathbb{R}} \prod_{i=1}^{n} f_{\mathbf{Y}_{i} \mid \omega_{i}}\left(\mathbf{Y}_{i} \mid \omega_{i} ; \boldsymbol{\beta}, \boldsymbol{\eta}\right) f_{\omega_{i}}\left(\omega_{i}, \nu\right) d \omega
$$

em que

$$
\begin{aligned}
\prod_{i=1}^{n} f_{Y_{i} \mid \omega_{i}}\left(\mathbf{Y}_{i} \mid \omega_{i} ; \boldsymbol{\beta}, \boldsymbol{\eta}\right) & =(2 \pi)^{-n / 2} \prod_{i=1}^{n}\left|\boldsymbol{\Sigma}_{i}\right|^{-1 / 2} \exp \left\{-\frac{1}{2}\left(\mathbf{Y}_{i}-\mathbf{X}_{i} \boldsymbol{\beta}\right)^{\top} \boldsymbol{\Sigma}_{i}^{-1}\left(\mathbf{Y}_{i}-\mathbf{X}_{i} \boldsymbol{\beta}\right)\right\} \\
& =(2 \pi)^{-n / 2}|\boldsymbol{\Sigma}|^{-1} \exp \left\{-\frac{1}{2}(\mathbf{Y}-\mathbf{X} \boldsymbol{\beta})^{\top} \boldsymbol{\Sigma}^{-1}(\mathbf{Y}-\mathbf{X} \boldsymbol{\beta})\right\}
\end{aligned}
$$


com $\mathbf{Y}=\left(\mathbf{Y}_{1}, \ldots, \mathbf{Y}_{n}\right)^{\top}$ sendo um vetor de dimensão $(m \times 1), \mathbf{X}=\left(\mathbf{X}_{1}, \ldots, \mathbf{X}_{n}\right)^{\top}$ é uma matriz por bloco de dimensão $(m \times p)$ e $\boldsymbol{\Sigma}=\operatorname{diag}\left\{\boldsymbol{\Sigma}_{1}, \ldots, \boldsymbol{\Sigma}_{n}\right\}$ uma matriz diagonal por bloco de dimensão $(m \times m)$, sendo $m=\sum_{i=1}^{n} m_{i}$. A função de verossimilhança dos efeitos aleatórios da escala pode ser expressa na forma

$$
\prod_{i=1}^{n} f_{\omega_{i}}(\boldsymbol{\omega} ; \nu)=\frac{(\nu / 2)^{n \nu / 2}}{\Gamma(\nu / 2)^{n}} \exp \left\{-\frac{\nu}{2} \sum_{i=1}^{n} \omega_{i}\right\} \prod_{i=1}^{n} \omega_{i}^{\nu / 2-1}
$$

Considerando a transformação proposta por Verbyla (1990), definimos a matriz $\mathbf{W}=\left[\mathbf{W}_{1}, \mathbf{W}_{2}\right]$ que satisfazem às condições

$$
\mathbf{W}_{1}^{\top} \mathbf{X}=\mathbf{I}_{p} \quad e \quad \mathbf{W}_{2}^{\top} \mathbf{X}=\mathbf{0},
$$

permitindo que a variável resposta $\mathbf{Y}$ condicionada a $\boldsymbol{\omega}$ seja transformada como $\mathbf{W}^{\top} \mathbf{Y}=\left[\mathbf{W}_{1}^{\top} \mathbf{Y}, \mathbf{W}_{2}^{\top} \mathbf{Y}\right]^{\top}$ $=\left[\mathbf{V}_{1}, \mathbf{V}_{2}\right]^{\top}$, tal que

$$
\mathbf{W}^{\top} \mathbf{Y} \mid \boldsymbol{\omega} \sim N_{m}\left(\mathbf{W}^{\top} \mathbf{X} \boldsymbol{\beta}, \mathbf{W}^{\top} \boldsymbol{\Sigma} \mathbf{W}\right)
$$

$\mathrm{e}$

$$
\left[\begin{array}{l}
\mathbf{V}_{1} \\
\mathbf{V}_{2}
\end{array}\right] \mid \boldsymbol{\omega} \sim N_{m}\left(\left[\begin{array}{c}
\boldsymbol{\beta} \\
\mathbf{0}
\end{array}\right],\left[\begin{array}{ll}
\mathbf{W}_{1}^{\top} \boldsymbol{\Sigma} \mathbf{W}_{1} & \mathbf{W}_{1}^{\top} \boldsymbol{\Sigma} \mathbf{W}_{2} \\
\mathbf{W}_{2}^{\top} \boldsymbol{\Sigma} \mathbf{W}_{1} & \mathbf{W}_{2}^{\top} \boldsymbol{\Sigma} \mathbf{W}_{2}
\end{array}\right]\right)
$$

Assim, a verossimilhança marginal de $\mathbf{Y}$ pode ser expressa como

$$
L(\boldsymbol{\lambda} ; \mathbf{Y})=\int_{\mathbb{R}^{n}} f_{\mathbf{V}_{1} \mid \mathbf{V}_{2}}\left(\mathbf{v}_{1} \mid \mathbf{v}_{2}, \boldsymbol{\omega} ; \boldsymbol{\beta}, \boldsymbol{\theta}\right) f_{\mathbf{V}_{2} \mid \boldsymbol{\omega}}\left(\mathbf{v}_{2} \mid \boldsymbol{\omega} ; \boldsymbol{\theta}\right) f_{\boldsymbol{\omega}}(\boldsymbol{\omega}, \nu) d \boldsymbol{\omega} .
$$

Da transformação proposta por Verbyla (1990) para determinar a verossimilhança restrita dos parâmetros de variância num modelo linear, temos que

$$
f_{\mathbf{V}_{1} \mid \mathbf{V}_{2}}\left(\mathbf{v}_{1} \mid \mathbf{v}_{2}, \boldsymbol{\omega} ; \boldsymbol{\beta}, \boldsymbol{\theta}\right)=(2 \pi)^{-p / 2}\left|\mathbf{X}^{\top} \boldsymbol{\Sigma}^{-1} \mathbf{X}\right|^{1 / 2} \exp \left\{-\frac{1}{2}(\mathbf{Y}-\mathbf{X} \boldsymbol{\beta})^{\top} \boldsymbol{\Sigma}^{-1}(\mathbf{Y}-\mathbf{X} \boldsymbol{\beta})+\frac{1}{2} \mathbf{Y}^{\top} \mathbf{P Y}\right\}
$$

$\mathrm{e}$

$$
f_{\mathbf{V}_{2} \mid \boldsymbol{\omega}}\left(\mathbf{v}_{2} \mid \boldsymbol{\omega} ; \boldsymbol{\theta}\right)=(2 \pi)^{-(n-p) / 2}|\boldsymbol{\Sigma}|^{-1 / 2}\left|\mathbf{X}^{\top} \boldsymbol{\Sigma}^{-1} \mathbf{X}\right|^{-1 / 2} \exp \left\{-\frac{1}{2} \mathbf{Y}^{\top} \mathbf{P} \mathbf{Y}\right\}
$$

em que

$$
\mathbf{P}=\mathbf{W}_{2}\left(\mathbf{W}_{2}^{\top} \boldsymbol{\Sigma} \mathbf{W}_{2}\right)^{-1} \mathbf{W}_{2}^{\top}=\boldsymbol{\Sigma}^{-1}-\boldsymbol{\Sigma}^{-1} \mathbf{X}\left(\mathbf{X}^{\top} \boldsymbol{\Sigma}^{-1} \mathbf{X}\right)^{-1} \mathbf{X}^{\top} \boldsymbol{\Sigma}^{-1}
$$

\subsection{Aproximação da verossimilhança marginal}

Considerando o modelo proposto em (5.1) e a formulação da distribuição condicional de $\mathbf{Y}_{i}$ dado $\omega_{i}$ em (5.2), podemos utilizar a aproximação parcial de Laplace para obter uma aproximação da verossimilhança marginal num modelo linear misto t-Student e decompor a aproximação na forma dada em (E.2), sendo

$$
\begin{aligned}
L_{1}(\boldsymbol{\lambda} ; \mathbf{Y})= & \left|\mathbf{I}_{n}+\left(\mathbf{V}-\widetilde{\mathbf{H}}^{* 2}-2 \widetilde{\mathbf{R}}_{c}^{\top} \widetilde{\mathbf{H}} \widetilde{\mathbf{R}}_{c}\right)^{-1}\left(\widetilde{\mathbf{H}}^{* 2}+2 \widetilde{\mathbf{R}}_{c}^{\top} \widetilde{\mathbf{H}} \widetilde{\mathbf{R}}_{c}\right)\right|^{-1 / 2} \\
& \exp \left[-\frac{1}{2}\left\{p \log (2 \pi)-\log \left|\mathbf{X}^{\top} \widetilde{\boldsymbol{\Sigma}}^{-1} \mathbf{X}\right|+(\mathbf{Y}-\mathbf{X} \boldsymbol{\beta})^{\top} \widetilde{\boldsymbol{\Sigma}}^{-1}(\mathbf{Y}-\mathbf{X} \boldsymbol{\beta})-\mathbf{Y}^{\top} \tilde{\mathbf{P}} \mathbf{Y}\right\}\right] \\
& \exp \left\{-\frac{1}{4\left(\nu-2+m_{i}\right)} \mathbf{1}^{\top}\left(\widetilde{\mathbf{H}}^{*}-\widetilde{\mathbf{D}}+\widetilde{\mathbf{D}}_{c}\right)^{\top}\left(\widetilde{\mathbf{H}}^{*}-\widetilde{\mathbf{D}}+\widetilde{\mathbf{D}}_{c}\right) \mathbf{1}\right\}
\end{aligned}
$$


$\mathrm{e}$

$$
\begin{aligned}
L_{2}(\boldsymbol{\eta} ; \mathbf{Y})= & \left.|\widetilde{\boldsymbol{\Sigma}}|^{-1 / 2}\left|\mathbf{X}^{\top} \widetilde{\boldsymbol{\Sigma}}^{-1} \mathbf{X}\right|^{-1 / 2}|| \widetilde{\mathbf{\Omega}}|| \frac{1}{2}\left(\mathbf{V}-\widetilde{\mathbf{H}}^{* 2}-2 \widetilde{\mathbf{R}}_{c}^{\top} \widetilde{\mathbf{H}} \widetilde{\mathbf{R}}_{c}\right)\right|^{-1 / 2}(2 \pi)^{-(n-p) / 2} \exp \left\{-\frac{1}{2} \mathbf{Y}^{\top} \tilde{\mathbf{P}} \mathbf{Y}\right\} \\
& \times \frac{(\nu / 2)^{n \nu / 2}}{\Gamma(\nu / 2)^{n}} \exp \left\{-\frac{\nu}{2} \sum_{i=1}^{n} \tilde{\omega}_{i}\right\} \prod_{i=1}^{n} \tilde{\omega}_{i}^{\nu / 2-1},
\end{aligned}
$$

em que $\mathbf{V}=\left(\nu-2+m_{i}\right) \mathbf{I}_{n}$ e $\boldsymbol{\Omega}=\operatorname{diag}\left\{w_{i}\right\}$ são matrizes diagonais de dimensão $n$. A matriz $\mathbf{H}$ é uma matriz quadrada de dimensão $m$ dada por $\boldsymbol{\Sigma}^{-1 / 2} \mathbf{X}\left(\mathbf{X}^{\top} \boldsymbol{\Sigma}^{-1} \mathbf{X}\right)^{-1} \mathbf{X}^{\top} \boldsymbol{\Sigma}^{-1 / 2}$ que pode ser particionada por blocos ou submatrizes $\mathbf{H}_{i j}$, com $i$ e $j$ de $1, \ldots, n$, dadas por

$$
\mathbf{H}_{i j}= \begin{cases}\boldsymbol{\Sigma}_{i}^{-1 / 2} \mathbf{X}_{i}\left(\mathbf{X}^{\top} \boldsymbol{\Sigma}^{-1} \mathbf{X}\right)^{-1} \mathbf{X}_{i}^{\top} \boldsymbol{\Sigma}_{i}^{-1 / 2} & \text { quando } i=j \\ \boldsymbol{\Sigma}_{i}^{-1 / 2} \mathbf{X}_{i}\left(\mathbf{X}^{\top} \boldsymbol{\Sigma}^{-1} \mathbf{X}\right)^{-1} \mathbf{X}_{j}^{\top} \boldsymbol{\Sigma}_{j}^{-1 / 2} & \text { quando } i \neq j .\end{cases}
$$

A matriz $\mathbf{H}^{* 2}$ é uma matriz quadrada de dimensão $n$ cujos elementos são dados por $\operatorname{tr}\left\{\mathbf{H}_{i j} \mathbf{H}_{j i}\right\}$, para todo $i, j$ de $1, \ldots, n$, e a matriz $\mathbf{H}^{*}$ é diagonal de dimensão $n$ com elementos $\operatorname{tr}\left\{\mathbf{H}_{i i}\right\}$.

O estimador de máxima verossimilhança para $\boldsymbol{\beta}$ da função de verossimilhança condicional é dado por $\hat{\boldsymbol{\beta}}_{c}=\left(\mathbf{X}^{\top} \boldsymbol{\Sigma}^{-1} \mathbf{X}\right)^{-1} \mathbf{X}^{\top} \boldsymbol{\Sigma}^{-1} \mathbf{Y}$ e as matrizes diagonais $\mathbf{D}, \mathbf{D}_{c}$ e $\mathbf{R}_{c}$ são definidas como:

$$
\begin{gathered}
\mathbf{D}=\operatorname{diag}\left\{\omega_{i}\left(\mathbf{Y}_{i}-\mathbf{X}_{i} \boldsymbol{\beta}\right)^{\top} \boldsymbol{\Sigma}_{i}(\boldsymbol{\theta})^{-1}\left(\mathbf{Y}_{i}-\mathbf{X}_{i} \boldsymbol{\beta}\right)\right\}=\operatorname{diag}\left\{\omega_{i} d_{i}\right\} \\
\mathbf{D}_{c}=\operatorname{diag}\left\{\omega_{i}\left(\mathbf{Y}_{i}-\mathbf{X}_{i} \hat{\boldsymbol{\beta}}_{c}\right)^{\top} \boldsymbol{\Sigma}_{i}(\boldsymbol{\theta})^{-1}\left(\mathbf{Y}_{i}-\mathbf{X}_{i} \hat{\boldsymbol{\beta}}_{c}\right)\right\}=\operatorname{diag}\left\{\omega_{i} d_{i, c}\right\} \mathrm{e} \\
\mathbf{R}_{c}=\operatorname{diag}\left\{\omega_{i}^{1 / 2} \boldsymbol{\Sigma}_{i}(\boldsymbol{\theta})^{-1 / 2}\left(\mathbf{Y}_{i}-\mathbf{X}_{i} \hat{\boldsymbol{\beta}}_{c}\right)\right\}=\operatorname{diag}\left\{\omega_{i}^{1 / 2} \boldsymbol{\Sigma}_{i}(\boldsymbol{\theta})^{-1 / 2} \mathbf{r}_{i c}\right\} \\
\mathbf{R}_{c}^{\top}=\operatorname{diag}\left\{\omega_{i}^{1 / 2} \mathbf{r}_{i c}^{\top} \boldsymbol{\Sigma}_{i}(\boldsymbol{\theta})^{-1 / 2}\right\}
\end{gathered}
$$

O símbolo $(\sim)$ indica que os termos são avaliados no máximo de $\boldsymbol{\omega}$ em $h_{2}(\cdot)$. Portanto, $\tilde{\boldsymbol{\omega}}$ é obtido da solução de $h_{2}^{\prime}(\cdot)=0$, dado por

$$
\tilde{\omega}_{i}=\frac{\nu-2+m_{i}-\operatorname{tr}\left(\widetilde{\mathbf{H}}_{i i}\right)}{\nu+\tilde{d}_{i, c}}
$$

para cada $i=1, \ldots, n$.

\subsubsection{Estimação dos efeitos fixos}

Sem considerar os termos constantes com respeito a $\boldsymbol{\beta}$, o logaritmo de $L_{1}(\boldsymbol{\lambda} ; \mathbf{Y})$ fica dado por

$$
l_{1}(\boldsymbol{\lambda} ; \mathbf{Y})=-\frac{1}{2}(\mathbf{Y}-\mathbf{X} \boldsymbol{\beta})^{\top} \widetilde{\boldsymbol{\Sigma}}^{-1}(\mathbf{Y}-\mathbf{X} \boldsymbol{\beta})+\frac{1}{4\left(\nu-2+m_{i}\right)} \mathbf{1}^{\top}\left(\widetilde{\mathbf{H}}^{*}-\widetilde{\mathbf{D}}+\widetilde{\mathbf{D}}_{c}\right)^{\top}\left(\widetilde{\mathbf{H}}^{*}-\widetilde{\mathbf{D}}+\widetilde{\mathbf{D}}_{c}\right) \mathbf{1}
$$

A derivada com respeito a $\boldsymbol{\beta}$ assume a forma

$$
\begin{aligned}
\frac{\partial l_{1}(\boldsymbol{\lambda} ; \mathbf{Y})}{\partial \boldsymbol{\beta}} & =\mathbf{X}^{\top} \widetilde{\boldsymbol{\Sigma}}^{-1}(\mathbf{Y}-\mathbf{X} \boldsymbol{\beta})-\frac{2}{4\left(\nu-2+m_{i}\right)} \mathbf{1}^{\top} \frac{\partial \widetilde{\mathbf{D}}}{\partial \boldsymbol{\beta}}\left(\widetilde{\mathbf{H}}^{*}-\widetilde{\mathbf{D}}+\widetilde{\mathbf{D}}_{c}\right) \mathbf{1} \\
& =-\sum_{i=1}^{n} \frac{\left(\nu+d_{i}\right)}{\nu-2+m_{i}} \tilde{\omega}_{i} \mathbf{X}_{i}^{\top} \widetilde{\boldsymbol{\Sigma}}_{i}^{-1}\left(\mathbf{Y}_{i}-\mathbf{X}_{i} \boldsymbol{\beta}\right) .
\end{aligned}
$$

Da equação (E.3) temos que $\left(\nu+d_{i}\right)=\left(\nu+m_{i}\right) / \bar{\omega}_{i}$, substituindo esta relação na equação anterior obtemos

$$
\frac{\partial l_{1}(\boldsymbol{\lambda} ; \mathbf{Y})}{\partial \boldsymbol{\beta}}=-\sum_{i=1}^{n}\left(\frac{\nu+m_{i}}{\nu-2+m_{i}}\right) \frac{\tilde{\omega}_{i}}{\bar{\omega}_{i}} \mathbf{X}_{i}^{\top} \widetilde{\boldsymbol{\Sigma}}_{i}^{-1}\left(\mathbf{Y}_{i}-\mathbf{X}_{i} \boldsymbol{\beta}\right)
$$


Igualando a zero, temos que o estimador para $\boldsymbol{\beta}$ obtido da verossimilhança condicional fica dado por

$$
\hat{\boldsymbol{\beta}}=\left(\mathbf{X}^{\top} \widetilde{\mathbf{U}} \mathbf{X}\right)^{-1} \mathbf{X}^{\top} \tilde{\mathbf{U}} \mathbf{Y}, \quad \text { em que } \quad \widetilde{\mathbf{U}}=\operatorname{diag}\left\{\left(\frac{\left(\nu+m_{i}\right) \tilde{\omega}_{i}}{\left(\nu-2+m_{i}\right) \bar{\omega}_{i}}\right) \widetilde{\boldsymbol{\Sigma}}_{i}^{-1}\right\} .
$$

Quando a dimensão das respostas $\mathbf{Y}_{i}$ é igual a 1, para todo $i=1, \ldots, n$, isto é, $m_{i}=1$, recuperamos a estrutura do modelo t-heteroscedástico proposto por Taylor e Verbyla (2006). Para $\nu \rightarrow \infty$ a distribuição t-Student é aproximada à distribuição normal. Neste caso, vemos que se $\nu \rightarrow \infty$, $\frac{\left(\nu+m_{i}\right)}{\left(\nu-2+m_{i}\right)} \rightarrow 1$, e $\bar{\omega}_{i} \rightarrow 1$ e $\hat{\boldsymbol{\beta}}$ tenderá ao estimador de máxima verossimilhança de $\boldsymbol{\beta}$ do caso normal.

\subsubsection{Estimação dos parâmetros de variância e covariância}

A função $L_{2}(\boldsymbol{\eta} ; \mathbf{Y})$ é utilizada para a estimação dos parâmetros de variância e covariância da matriz $\boldsymbol{\Sigma}(\boldsymbol{\theta})$. Omitindo constantes, o logaritmo de $L_{2}(\boldsymbol{\eta} ; \mathbf{Y})$ fica dado por

$$
\begin{aligned}
l_{2}(\boldsymbol{\theta} ; \mathbf{Y})= & -\frac{1}{2} \log |\widetilde{\boldsymbol{\Sigma}}|-\frac{1}{2} \log \left|\mathbf{X}^{\top} \widetilde{\boldsymbol{\Sigma}}^{-1} \mathbf{X}\right|-\frac{1}{2} \log \left|\frac{1}{2}\left(\mathbf{V}-\widetilde{\mathbf{H}}^{* 2}-2 \widetilde{\mathbf{R}}_{c}^{\top} \widetilde{\mathbf{H}} \widetilde{\mathbf{R}}_{c}\right)\right|-\frac{1}{2} \mathbf{Y}^{\top} \widetilde{\mathbf{P}} \mathbf{Y} \\
& -\frac{\nu}{2} \sum_{i=1}^{n} \tilde{\omega}_{i}+(\nu / 2-1) \sum_{i=1}^{n} \log \tilde{\omega}_{i} .
\end{aligned}
$$

Seja $\widetilde{\mathbf{Q}}=\frac{1}{2}\left(\mathbf{V}-\widetilde{\mathbf{H}}^{* 2}-2 \widetilde{\mathbf{R}}_{c}^{\top} \widetilde{\mathbf{H}} \widetilde{\mathbf{R}}_{c}\right)$ uma função dos parâmetros de variância-covariância que pode ser seminegativa definida. Para garantir que $\widetilde{\mathbf{Q}}$ seja definida positiva, os componentes observados da resposta podem ser eliminados considerando o valor esperado desta matriz. Para $\tilde{\boldsymbol{\omega}}$ fixo, a esperança condicional de $\tilde{\mathbf{Q}}$ é dada por

$$
\mathbf{Q}^{*}=\mathrm{E}\{\widetilde{\mathbf{Q}} \mid \widetilde{\boldsymbol{\omega}}=\overline{\boldsymbol{\omega}}\}=\mathrm{E}\left\{\frac{1}{2}\left(\mathbf{V}-\mathbf{H}^{* 2}-2 \mathbf{R}_{c}^{\top} \mathbf{H R}_{c} \mid \overline{\boldsymbol{\omega}}\right)\right\}=\frac{1}{2}\left(\mathbf{V}-\mathbf{H}^{* 2}\right)-\mathrm{E}\left\{\mathbf{R}_{c}^{\top} \mathbf{H} \mathbf{R}_{c} \mid \overline{\boldsymbol{\omega}}\right\}
$$

em que o $(i, j)$-ésimo elemento da matriz $\mathbf{R}_{c}^{\top} \mathbf{H} \mathbf{R}_{c}$ fica dado por $\left(\omega_{i} \omega_{j}\right)^{1 / 2}\left(\mathbf{Y}_{i}-\mathbf{X}_{i} \hat{\boldsymbol{\beta}}_{c}\right)^{\top} \boldsymbol{\Sigma}_{i}^{-1 / 2}(\boldsymbol{\theta}) \mathbf{H}_{i j}$ $\boldsymbol{\Sigma}_{j}^{-1 / 2}\left(\mathbf{Y}_{j}-\mathbf{X}_{j} \hat{\boldsymbol{\beta}}_{c}\right)$, para $i, j=1, \ldots, n$. Então, podemos obter o valor esperado para cada elemento da matriz $\mathbf{R}_{c}^{\top} \mathbf{H} \mathbf{R}_{c}$

$$
\begin{aligned}
\mathrm{E}\left[\mathbf{R}_{c}^{\top} \mathbf{H} \mathbf{R}_{c} \mid \overline{\boldsymbol{\omega}}\right] & =\mathrm{E}\left\{\left(\omega_{i} \omega_{j}\right)^{1 / 2}\left(\mathbf{Y}_{i}-\mathbf{X}_{i} \hat{\boldsymbol{\beta}}_{c}\right)^{\top} \boldsymbol{\Sigma}_{i}^{-1 / 2}(\boldsymbol{\theta}) \mathbf{H}_{i j} \boldsymbol{\Sigma}_{j}^{-1 / 2}(\boldsymbol{\theta})\left(\mathbf{Y}_{j}-\mathbf{X}_{j} \hat{\boldsymbol{\beta}}_{c}\right)\right\} \\
& =\left(\omega_{i} \omega_{j}\right)^{1 / 2} \operatorname{tr}\left\{\boldsymbol{\Sigma}_{i}^{-1 / 2}(\boldsymbol{\theta}) \mathbf{H}_{i j} \boldsymbol{\Sigma}_{j}^{-1 / 2}(\boldsymbol{\theta}) \mathrm{E}\left(\mathbf{r}_{i, c} \mathbf{r}_{j, c}^{\top}\right)\right\}
\end{aligned}
$$

resultando em

$$
\left(\omega_{i} \omega_{j}\right)^{1 / 2} \operatorname{tr}\left\{\boldsymbol{\Sigma}_{i}^{-1 / 2}(\boldsymbol{\theta}) \mathbf{H}_{i j} \boldsymbol{\Sigma}_{j}^{-1 / 2}(\boldsymbol{\theta}) \mathrm{E}\left(\mathbf{r}_{i, c} \mathbf{r}_{j, c}^{\top}\right)\right\}=\left\{\begin{array}{cc}
\operatorname{tr}\left(\mathbf{H}_{i i}\right)-\operatorname{tr}\left(\mathbf{H}_{i i}^{2}\right) & \text { quando } i=j \\
-\operatorname{tr}\left(\mathbf{H}_{i j} \mathbf{H}_{j i}\right) & \text { quando } i \neq j
\end{array}\right.
$$

Portanto a matriz $\widetilde{\mathbf{Q}}^{*}$ fica dada por

$$
\widetilde{\mathbf{Q}}^{*}=\frac{1}{2}\left(\mathbf{V}+\widetilde{\mathbf{H}}^{* 2}-2 \widetilde{\mathbf{H}}^{*}\right),
$$

pode ser sustituido em $l_{2}(\boldsymbol{\eta} ; \mathbf{Y})$.

\subsection{Comentários}

Neste capítulo apresentamos uma extensão da aproximação de MVR proposta por Taylor e Verbyla (2006) para um modelo linear heteroscedástico t-Student para um modelo misto linear t-Student. Para isso, consideramos a formulação do modelo misto linear t-Student proposta por Pinheiro et al. 
(2001). Desta forma determinamos as estruturas matriciais das expressões para definir as verossimilhanças marginais dos parâmetros de interesse. Dada a formulação do modelo não podemos estender os resultados para toda a família elíptica, mas sim, pode ser utilizada para as distribuições de mistura de escala normal. Assim, por exemplo, podemos considerar o caso a distribuição exponencial potência como foi definida na Seção 3.6.2 do Capítulo 3. 


\section{Capítulo 6}

\section{Simulações e Aplicações}

Neste capítulo apresentamos simulações e aplicações de diferentes modelos linear t-Student e misto linear t-Student. Analisaremos as estimativas dos parâmetros obtidos pelos distintos métodos de estimação que foram propostos na Seção 3.6.1 para o modelo linear t-Student e na Seção 4.3.2 para o modelo misto linear t-Student. Vamos considerar algumas das estruturas da matriz $\boldsymbol{\Sigma}$ descritas na Seção 3.5 e detalhadas na Seção B.3 do Apêndice B.

Na Seção 6.1 apresentaremos simulações considerando o modelo linear t-Student com duas estruturas para a matriz $\boldsymbol{\Sigma}$. Enquanto que na Seção 6.2 analisaremos para o modelo misto linear t-Student, três modelos com a mesma estrutura para a matriz $\boldsymbol{\Sigma}$, mas com distintas estruturas para a matriz $\mathbf{X}$ em dados balanceados e não balanceados. Para todos os casos de simulação fixaremos três tamanhos de amostra 20, 50 e 100 e três valores para os graus de liberdade 4, 8 e 15 e realizaremos 500 repetições. As amostras foram geradas considerando a representação estocástica de uma distribuição elíptica (Apéndice C).

Tanto o processo de simulação quanto as aplicações foram desenvolvidas na linguagem de programação R Core Team (2018).

\subsection{Simulações do modelo linear t-Student}

Na Seção 3.6.1 vimos que no modelo linear t-Student o método de máxima verossimilhança restrita coincide com o método de máxima verossimilhança perfilada modificada. Portanto, vamos comparar apenas três estimativas do vetor de parâmetros $\alpha$ que define a estrutura de $\boldsymbol{\Sigma}$ e por consequência as estimativas de $\boldsymbol{\beta}$.

\subsubsection{Estrutura simples para a matriz escala}

Considerando a estrutura mais simples da matriz $\boldsymbol{\Sigma}$ dada por $\sigma^{2} \mathbf{I}_{m}$ no modelo linear t-Student (ver expressões na Seção 3.6.1) temos que as expressões dos estimadores de $\sigma^{2}$ são explícitas e que o único estimador que depende dos graus de liberdade é pelo método de máxima verossimilhança perfilada modificada aproximada. Também da equação (3.23) temos que a estimativa de $\boldsymbol{\beta}$ não depende de $\sigma^{2}$. Na Tabela 6.1, fixando os graus de liberdade em $\nu=4$, apresentamos os resultados obtidos por 500 simulações considerando o verdadeiro valor de $\sigma^{2}$ igual a 1 .

Na Tabela 6.1, o viés relativo é a diferença relativa em valor absoluto entre a média das estimativas e o valor verdadeiro de $\sigma^{2}$ e podemos notar uma superioridade do método de máxima verossimilhança restrita com relação ao método de máxima verossimilhança e máxima verossimilhança modificada ajustada. 
Tabela 6.1: Estimativas de $\sigma^{2}$ obtidas pelos três métodos de estimação, para $\sigma^{2}=1$.

\begin{tabular}{|c|c|c|c|c|c|c|c|c|c|}
\hline$m$ & \multicolumn{3}{|c|}{20} & \multicolumn{3}{|c|}{50} & \multicolumn{3}{|c|}{100} \\
\hline Método & MV & MVR & MVPMA & MV & MVR & MVPMA & MV & MVR & MVPMA \\
\hline Média & 0.8958 & 0.9953 & 1.4928 & 0.9543 & 0.9941 & 1.2426 & 0.9781 & 0.9978 & 1.4971 \\
\hline Desvio padrão & 0.2634 & 0.2928 & 0.4392 & 0.1700 & 0.1771 & 0.2213 & 0.1224 & 0.1249 & 0.1873 \\
\hline Viés relativo & 0.1041 & 0.0046 & 0.4928 & 0.0456 & 0.0058 & 0.2426 & 0.0219 & 0.0021 & 0.4971 \\
\hline
\end{tabular}

\subsubsection{Estrutura autoregressiva para a matriz escala}

Na Seção D.3.1 do Apêndice D explicamos brevemente o processo de estimação para cada método e especificamos o logaritmo da função de verossimilhança, a função de verossimilhança restrita e as funções de verossimilhança perfilada modificada e modificada aproximada para a estrutura de $\boldsymbol{\Sigma}$ igual a $\sigma^{2} \mathbf{R}$. Para evitar problemas com inversa de $\boldsymbol{\Sigma}$ no processo de maximização das funções, vamos considerar a decomposição da matriz inversa de $\mathbf{R}$ como $\mathbf{R}^{-1}=\mathbf{Q}^{\top} \mathbf{Q}$, em que a matriz $\mathbf{Q}$, tem a mesma estrutura utilizada por Özkale e Acar (2015) e é dada por

$$
\mathbf{Q}=\left[\begin{array}{cccccc}
\sqrt{\left(1-\rho^{2}\right)} & 0 & 0 & \ldots & 0 & 0 \\
-\rho & 1 & 0 & \ldots & 0 & 0 \\
0 & -\rho & 1 & \ldots & 0 & 0 \\
\vdots & \vdots & \vdots & \ddots & \vdots & \vdots \\
0 & 0 & 0 & \ldots & 1 & 0 \\
0 & 0 & 0 & \ldots & -\rho & 1
\end{array}\right]
$$

Desta forma, podemos simplificar alguns termos das funções devido a que

- $\left|\mathbf{R}^{-1}\right|=\left|\mathbf{Q}^{\top} \mathbf{Q}\right|=|\mathbf{Q}|^{2}=1-\rho^{2}$, e о $|\mathbf{R}|=\frac{1}{1-\rho^{2}}$.

- A forma quadrática de $u=(\mathbf{Y}-\mathbf{X} \boldsymbol{\beta})^{\top} \mathbf{R}^{-1}(\mathbf{Y}-\mathbf{X} \boldsymbol{\beta})$ pode ser escrita na forma $u=$ $(\mathbf{Y}-\mathbf{X} \boldsymbol{\beta})^{\top} \mathbf{Q}^{\top} \mathbf{Q}(\mathbf{Y}-\mathbf{X} \boldsymbol{\beta})=\left(\mathbf{Y}^{*}-\mathbf{X}^{*} \boldsymbol{\beta}\right)^{\top}\left(\mathbf{Y}^{*}-\mathbf{X}^{*} \boldsymbol{\beta}\right), \operatorname{com} \mathbf{Y}^{*}=\mathbf{Q} \mathbf{Y}$ e $\mathbf{X}^{*}=\mathbf{Q X}$.

\section{Resultados obtidos}

Para gerar as amostras consideramos os seguintes valores, o vetor $\boldsymbol{\alpha}=\left(\sigma^{2}, \rho\right)$ é dado por $\boldsymbol{\alpha}=(1,0.5)$ e o vetor $\boldsymbol{\beta}$ dado por $\boldsymbol{\beta}=(0.4,1.5)$. Na Figura 6.1 apresentamos os resultados das estimativas de $\sigma^{2}$ e $\rho$ obtidas por cada método de estimação para distintos valores de $\nu$ e $m$. Os gráficos estão ordenados por linha, em que cada linha representa um método de estimação e cada gráfico na linha representa o tamanho de amostra. Dentro de cada gráfico apresentamos as estimativas para os distintos graus de liberdade considerados na geração das amostras. Assim, se observamos cada gráfico vemos que à medida que aumentam os graus de liberdade a variabilidade das estimações fica menor. Por outro lado, se observamos cada linha notamos que não temos uma grande variação devido ao aumento do tamanho de amostra (não há uma grande variação na escala dos gráficos). Por último, observamos que as estimativas que apresentam mais discordância do verdadeiro valor são as obtidas por máxima verossimilhança perfilada modificada aproximada. Na Tabela 6.2 apresentamos as estimativas de $\sigma^{2}$ obtidas pelos distintos métodos de estimação e pelos diferentes esquemas de simulação. 

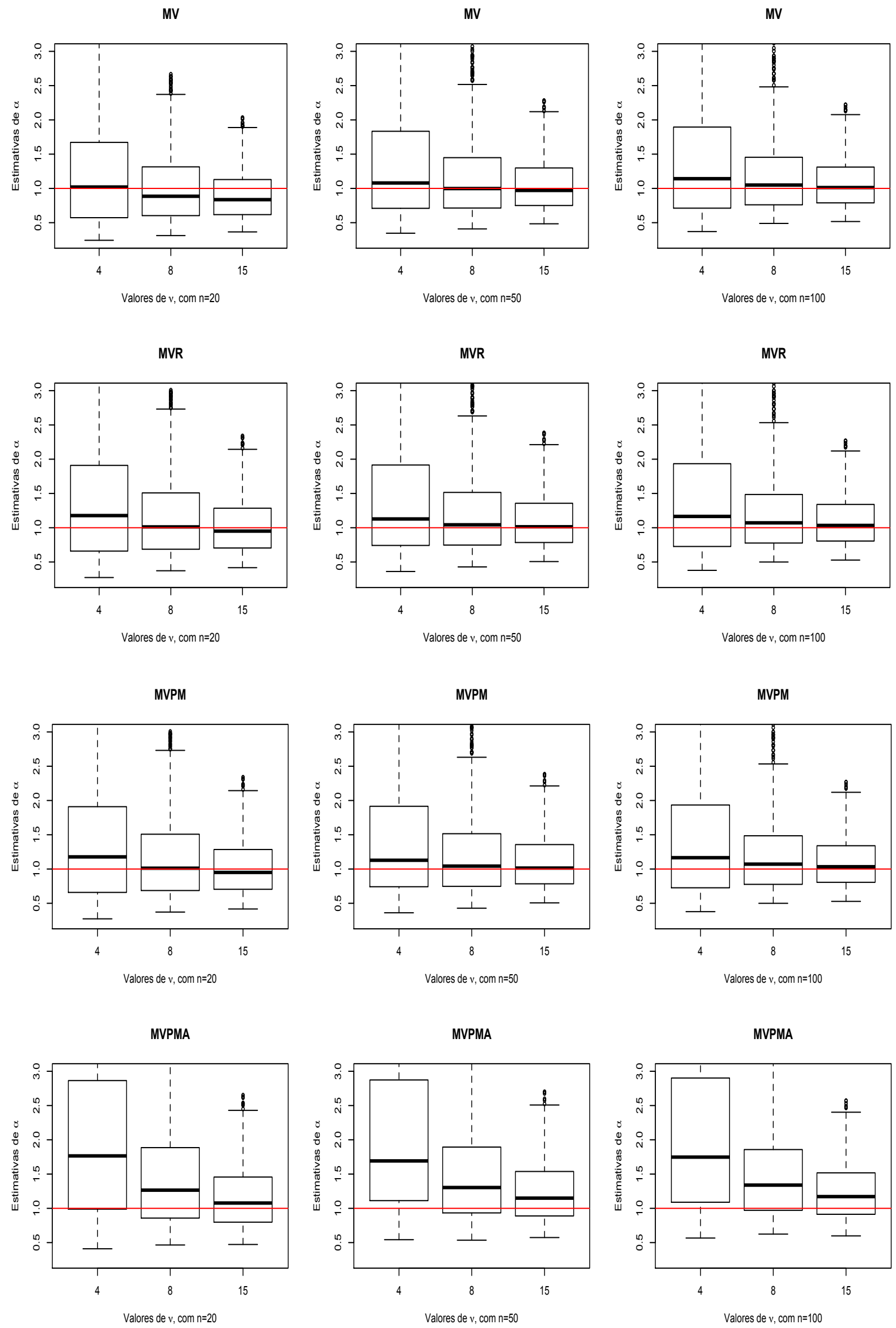

Figura 6.1: Gráficos boxplots para as estimativas de $\sigma^{2}$, considerando um modelo linear $t$-Student com estrutura autoregressiva para a matriz escala, os verdadeiros valores para $\sigma^{2}=1$ e $\rho=0.5$. 
Tabela 6.2: Estimativas de $\sigma^{2}$ obtidas por diferentes métodos de estimação, considerando o valor de $\sigma^{2}=1$.

\begin{tabular}{|c|c|c|c|c|c|c|c|c|c|c|c|c|c|}
\hline & \multicolumn{4}{|c|}{$\nu=4$} & \multicolumn{4}{|c|}{$\nu=8$} & \multicolumn{4}{|c|}{$\nu=15$} \\
\hline & & MV & MVR & MVPM & MVPMA & MV & MVR & MVPM & MVPMA & MV & MVR & MVPM & MVPMA \\
\hline \multirow{3}{*}{$m=20$} & Média & 1.2803 & 1.4632 & 1.4632 & 2.1957 & 0.9655 & 1.1031 & 1.1031 & 1.3789 & 0.8720 & 0.9951 & 0.9951 & 1.1277 \\
\hline & Desvio padrão & 0.8188 & 0.9372 & 0.9372 & 1.4068 & 0.4402 & 0.5036 & 0.5036 & 0.6295 & 0.2955 & 0.3370 & 0.3370 & 0.3819 \\
\hline & Viés relativo & 0.2803 & 0.4632 & 0.4632 & 1.1957 & 0.0345 & 0.1031 & 0.1031 & 0.3789 & 0.1280 & 0.0049 & 0.0049 & 0.1277 \\
\hline \multirow{3}{*}{$m=50$} & Média & 1.2930 & 1.3516 & 1.3516 & 2.0273 & 1.1250 & 1.1761 & 1.1761 & 1.4697 & 1.0738 & 1.1223 & 1.1223 & 1.2720 \\
\hline & Desvio padrão & 0.7457 & 0.7799 & 0.7799 & 1.1696 & 0.4705 & 0.4918 & 0.4918 & 0.6142 & 0.3315 & 0.3466 & 0.3466 & 0.3929 \\
\hline & Viés relativo & 0.2930 & 0.3516 & 0.3516 & 1.0273 & 0.1250 & 0.1761 & 0.1761 & 0.4697 & 0.0738 & 0.1223 & 0.1223 & 0.2720 \\
\hline \multirow{3}{*}{$m=100$} & Média & 1.3758 & 1.4051 & 1.4051 & 2.1076 & 1.1205 & 1.1439 & 1.1439 & 1.4306 & 1.0691 & 1.0918 & 1.0918 & 1.2373 \\
\hline & Desvio padrão & 0.7988 & 0.8155 & 0.8155 & 1.2233 & 0.3932 & 0.4007 & 0.4007 & 0.5022 & 0.3015 & 0.3078 & 0.3078 & 0.3489 \\
\hline & Viés relativo & 0.3758 & 0.4051 & 0.4051 & 1.1076 & 0.1205 & 0.1439 & 0.1439 & 0.4306 & 0.0691 & 0.0918 & 0.0918 & 0.2373 \\
\hline
\end{tabular}

Tabela 6.3: Estimativas de $\rho$ obtidas por diferentes métodos de estimação, considerando o valor de $\rho=0.5$.

\begin{tabular}{|c|c|c|c|c|c|c|c|c|c|c|c|c|c|}
\hline & \multicolumn{4}{|c|}{$\nu=4$} & \multicolumn{4}{|c|}{$\nu=8$} & \multicolumn{4}{|c|}{$\nu=15$} \\
\hline & & MV & MVR & MVPM & MVPMA & MV & MVR & MVPM & MVPMA & MV & MVR & MVPM & MVPMA \\
\hline \multirow{3}{*}{$m=20$} & Média & 0.287 & 0.460 & 0.460 & 0.460 & 0.282 & 0.455 & 0.455 & 0.455 & 0.288 & 0.461 & 0.461 & 0.461 \\
\hline & Desvio padrão & 0.221 & 0.264 & 0.264 & 0.265 & 0.225 & 0.270 & 0.270 & 0.271 & 0.224 & 0.269 & 0.269 & 0.269 \\
\hline & Viés relativo & 0.427 & 0.081 & 0.081 & 0.081 & 0.435 & 0.090 & 0.090 & 0.090 & 0.424 & 0.077 & 0.077 & 0.077 \\
\hline \multirow{3}{*}{$m=50$} & Média & 0.419 & 0.482 & 0.482 & 0.482 & 0.417 & 0.479 & 0.479 & 179 & 0.418 & 0.480 & 0.480 & 0.480 \\
\hline & Desvio padrão & 0.132 & 0.139 & 0.139 & & 0.132 & 0.139 & 0.139 & 39 & 0.133 & 0.139 & 0.139 & 0.139 \\
\hline & Viés relativo & 0.162 & 0.037 & 0.037 & 0.037 & 0.167 & 0.042 & 0.042 & 0.042 & 0.164 & 0.040 & 0.040 & 0.040 \\
\hline \multirow{3}{*}{$m=100$} & Média & 0.460 & 0.490 & 0.490 & 0.490 & 0.462 & 0.493 & 0.493 & 0.493 & 0.458 & 0.488 & 0.488 & 0.488 \\
\hline & Desvio padrão & 0.090 & 0.092 & 0.092 & 0.092 & 0.091 & 0.093 & 0.093 & 0.093 & 0.091 & 0.093 & 0.093 & 0.093 \\
\hline & Viés relativo & 0.080 & 0.019 & 0.019 & 0.019 & 0.075 & 0.014 & 0.014 & 0.014 & 0.084 & 0.023 & 0.023 & 0.0230 \\
\hline
\end{tabular}


Na Figura 6.2 apresentamos as estimativas do parâmetro $\rho$ observando que não existe maior variabilidade para os diferentes métodos de estimação. Na Tabela 6.3 apresentamos os valores das estimativas de $\rho$ para os diferentes métodos de estimação. Estas estimativas apresentam menor viés relativo à medida que o tamanho da amostra é maior.

\subsection{Simulações para o modelo misto linear t-Student}

As especificações do modelo misto linear t-Student foram dadas na Seção 4.3 .2 e vamos considerar para estrutura da matriz $\boldsymbol{\Sigma}_{i}$ a matriz $\alpha \mathbf{I}_{m i}+\gamma \mathbf{J}_{m i}$, em que o parâmetro $\alpha$ é associado ao vetor de erros $\boldsymbol{\epsilon}_{i}$ e $\gamma$ é associado ao vetor de efeitos aleatórios. Na Seção D.3.2 do Apêndice D explicamos brevemente o algoritmo de estimação.

Considerando esta estrutura de $\boldsymbol{\Sigma}$ temos um limitante para a correção proposta para a aplicação do método de máxima verosimilhança restrita da transformação de cada $\mathbf{Y}_{i}$, para $i=$ $1, \ldots, n$, obtemos a forma quadrática $u_{i 2}=\left(\mathbf{Y}_{i}-\mathbf{X}_{i} \mathbf{b}_{i}\right)^{\top} \boldsymbol{\Sigma}_{i}^{-1}\left(\mathbf{Y}_{i}-\mathbf{X}_{i} \mathbf{b}_{i}\right)$, em que $\mathbf{b}_{i}$ é dado por $\left(\mathbf{X}_{i}^{\top} \boldsymbol{\Sigma}_{i}^{-1} \mathbf{X}_{i}\right)^{-1} \mathbf{X}_{i}^{\top} \boldsymbol{\Sigma}_{i}^{-1} \mathbf{Y}_{i}$ (ver Seção 4.2.2). Podemos escrever $u_{i 2}$ na seguinte forma:

$$
u_{i 2}=\mathbf{Y}_{i}^{\top} \boldsymbol{\Sigma}_{i}^{-1 / 2}\left(\mathbf{I}_{m i}-\mathbf{H}_{i}\right) \boldsymbol{\Sigma}_{i}^{-1 / 2} \mathbf{Y}_{i}
$$

em que a matriz $\mathbf{H}_{i}$ é dada por $\boldsymbol{\Sigma}_{i}^{-1 / 2} \mathbf{X}_{i}\left(\mathbf{X}_{i}^{\top} \boldsymbol{\Sigma}_{i}^{-1} \mathbf{X}_{i}\right)^{-1} \mathbf{X}_{i}^{\top} \boldsymbol{\Sigma}_{i}^{-1 / 2}$. Um aspecto importante é que devemos assegurar que existe a inversa de $\left(\mathbf{X}_{i}^{\top} \boldsymbol{\Sigma}_{i}^{-1} \mathbf{X}_{i}\right)$, para $i=1, \ldots, n$, isto geralmente não é possível. Outra consideração é se $\mathbf{H}_{i}$ for a matriz identidade $\mathbf{I}_{m i}$, a transformação proposta não tem sentido pois o $u_{i 2}$ resultante é igual a zero. Este último resultado pode ser obtido quando $\mathbf{X}_{i}$ é igual a uma matriz identidade $\mathbf{I}_{m i}$, para $i=1, \ldots, n$.

Vamos considerar um modelo balanceado com a mesma estrutura de $\boldsymbol{\Sigma}$ e comparamos as estimativas obtidas por máxima verossimilhança e os ajustes da verossimilhança perfilada modificada. Também comparamos os resultados com as estimativas obtidas pela biblioteca heavy de Osorio (2018) no software R. Apresentamos os resultados obtidos de 1000 amostras geradas considerando os valores de $\alpha=5$ e $\gamma=5$.

\subsubsection{Modelo misto linear t-Student balanceado}

Nesta seção, consideramos um modelo balanceado, isto é, que todos os grupos contêm a mesma quantidade de medidas, $m_{i}$ igual a $m$. A matriz $\mathbf{X}_{i}$ é igual a $x_{i} \mathbf{1}_{m}$, em que $x_{i}$ é escalar e a matriz $\mathbf{Z}_{i}$ continua sendo um vetor de uns mas neste modelo tem dimensão $(m \times 1)$, para $i=1, \ldots, n$. Então, o modelo para cada resposta $\mathbf{Y}_{i}$ fica dado por

$$
\mathbf{Y}_{i}=\beta x_{i} \mathbf{1}_{m}+d_{i} \mathbf{1}_{m}+\boldsymbol{\epsilon}_{i}, \quad i=1, \ldots, n,
$$

em que o efeito aleatório $\mathbf{d}_{i}$ é uma variável aleatória univariada e $\boldsymbol{\epsilon}_{i}$ é um vetor aleatório de dimensão $(m \times 1)$. Então, cada resposta pode ser escrita da seguinte forma:

$$
\mathbf{Y}_{i}=\beta x_{i} \mathbf{1}_{m}+\left[\mathbf{1}_{m}, \mathbf{I}_{m}\right]\left[d_{i}, \boldsymbol{\epsilon}_{i}\right]^{\top},
$$

com $i=1, \cdots, n$. Supondo que o vetor $\left[d_{i}, \boldsymbol{\epsilon}_{i}\right]_{(m+1 \times 1)}^{\top}$ tem distribuição t-Student multivariada com parâmetro de posição igual a $\mathbf{0}$ e matriz de escala na forma

$$
\boldsymbol{\Sigma}_{i}^{*}=\left[\begin{array}{cc}
\gamma & \mathbf{0}^{\top} \\
\mathbf{0} & \alpha \mathbf{I}_{m}
\end{array}\right] .
$$

Então, para gerar as amostras vamos considerar que $\mathbf{Y}_{i}$ tem distribuição t-Student multivariada com parâmetro de posição $x_{i} \beta \mathbf{1}_{m}$ e matriz de escala dada por $\boldsymbol{\Sigma}_{i}=\alpha \mathbf{I}_{m}+\gamma \mathbf{J}_{m}$.

Considerando $m$ igual a 5 e os tamanhos da amostra (quantidade de indivíduos ou grupos) 25, 100, 200 e 800 e os graus de liberdade $\nu$ iguais a 4, 30 e 100. Para os valores dos parâmetros 
consideramos $\beta$ igual a $2, \alpha$ e $\gamma$ iguais a 5 . Os valores de $x_{i}$ foram gerados de uma distribuição uniforme no intervalo $[-2 ; 2]$, para $i=1, \cdots, n$.

\section{Resultados obtidos}

Os gráficos apresentados na Figura 6.3 mostram as estimativas de $\alpha$. Cada linha representa um método de estimação e cada gráfico na linha representa o tamanho da amostra. Não observamos grandes diferenças entre os gráficos das estimativas obtidas pelos diferentes métodos. Mas observamos que à medida que o tamanho de amostra e os graus de liberdade aumentam a variabilidade das estimativas diminui.

Na Figura 6.4 vemos que as estimativas de $\gamma$ obtidas pelos diferentes métodos têm maior variabilidade que as estimativas de $\alpha$. Nestas estimativas, também observamos uma diminuição da variabilidade à medida que o tamanho da amostra aumenta. Para o aumento dos graus de liberdade vemos um efeito menor na variabilidade das estimativas obtidas pelos diferentes métodos para o parâmetro $\gamma$.

Na Tabela 6.4, vemos que não há grande diferença entre os viéses relativos das estimativas. Da mesma forma que observamos na Figura 6.3, vemos que há um efeito dos tamanhos de amostra e graus de liberdade na variabilidade das estimativas obtidas pelos diferentes métodos de estimação. As diferenças das estimativas obtidas para $\alpha$, na Tabela 6.4, vemos que o viés relativo das estimativas de $\gamma$ tende a ser menor no método de máxima verossimilhança perfilada modificada do que nos outros métodos. Por último, vemos que em todos os casos as estimativas obtidas para $\gamma$ têm maior variabilidade do que as estimativas obtidas para $\alpha$.

Na Tabela 6.5 apresentamos os viéses relativos das estimativas de $\alpha$ e $\gamma$ para comparar o ajuste considerando uma t-Student e um modelo misto linear normal. Os dados foram gerados de um modelo misto linear t-Student com 4 graus de liberdade. Vemos que os viéses relativos do ajuste normal ficam próximos de um, isto é, que as médias das estimativas obtidas pelos métodos são o dobro do verdadeiro valor do parâmetro. Os viéses relativos do ajuste t-Student são menores do que 0.030 e diminuem à medida que o tamanho da amostra aumenta.

$\mathrm{Na}$ Tabela 6.6 da mesma forma que a tabela anterior apresentamos os viéses relativos das estimativas de $\alpha$ e $\gamma$ para comparar o ajuste considerando uma t-Student e um modelo misto linear normal. Mas os dados foram gerados considerando um modelo misto linear normal. Neste caso o ajuste normal apresenta um melhor comportamento, no caso do viéses relativos das estimativas de $\alpha$ estão em torno de zero e das estimativas de $\gamma$ são menores do que 0.055 e diminuem à medida que aumenta o tamanho dos grupos. Os viéses relativos do ajuste t-Student são maiores que os viéses do ajuste normal, mas também diminuem à medida que o tamanho dos grupos aumenta. A diferença na Tabela 6.5 é que não há maiores diferenças entre os métodos de estimação. Neste caso os viéses relativos de máxima verossimilhança perfilada modificada são menores do que de máxima verossimilhança. 

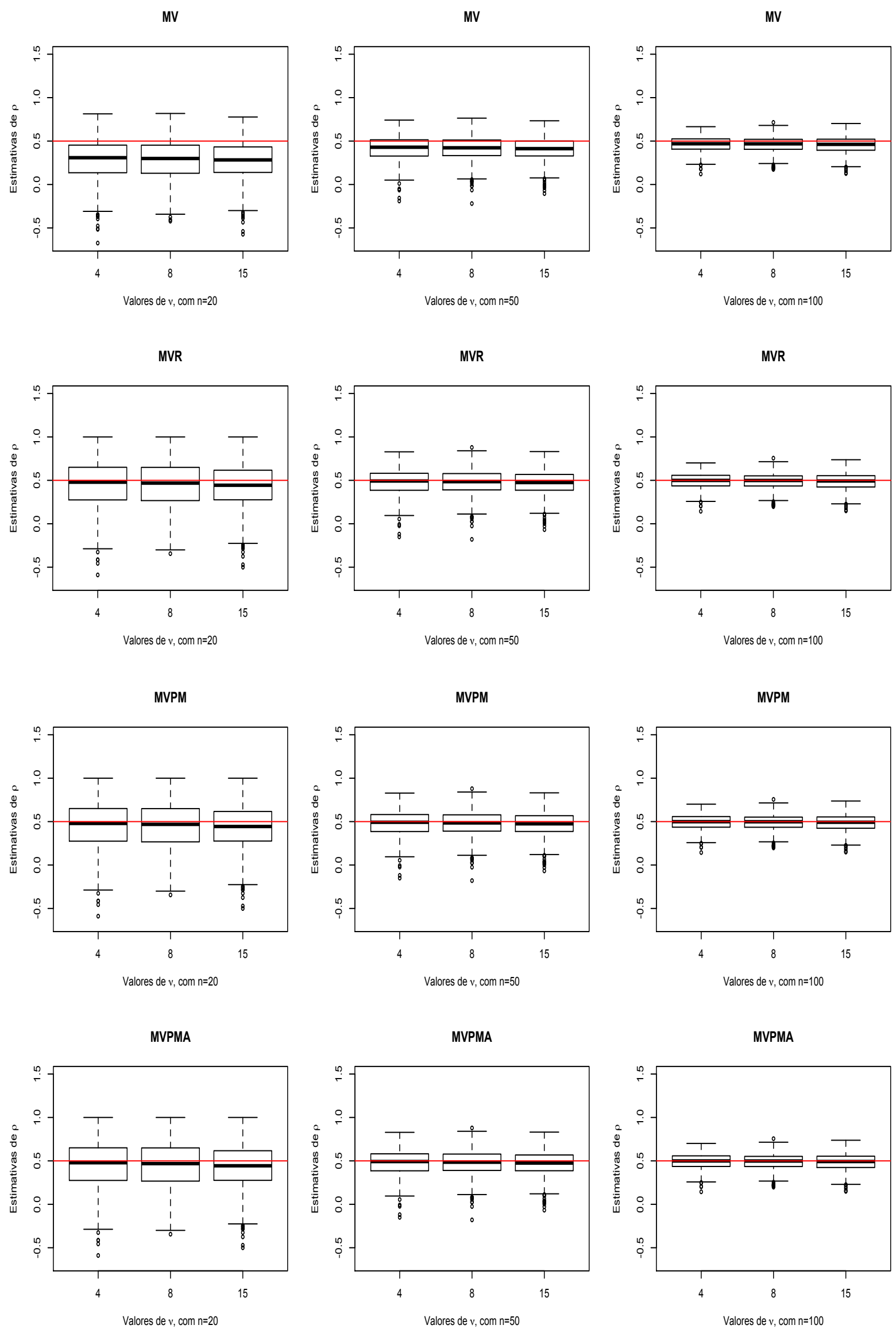

Figura 6.2: Gráficos boxplots para as estimativas de $\rho$, considerando um modelo linear $t$-Student com estrutura autoregressiva para a matriz escala, os verdadeiros valores para $\sigma^{2}=1$ e $\rho=0.5$. 
MV-heavy

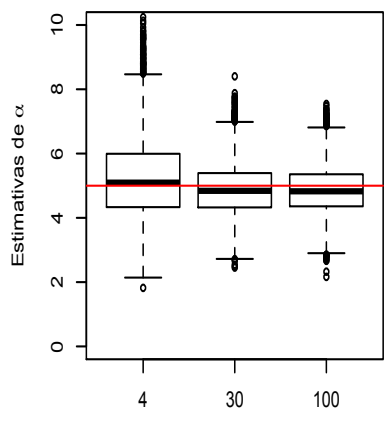

Valores de $v, \operatorname{com} n=25$
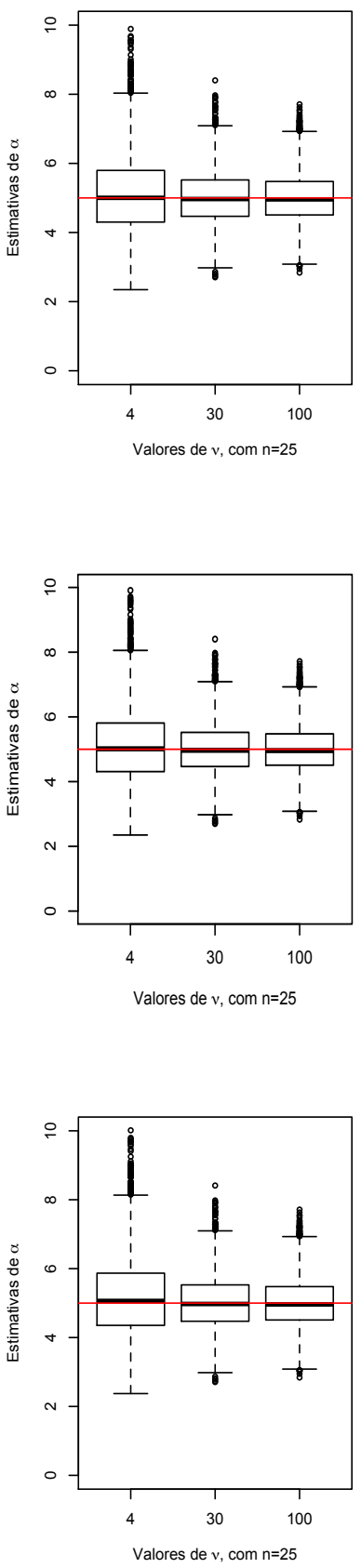
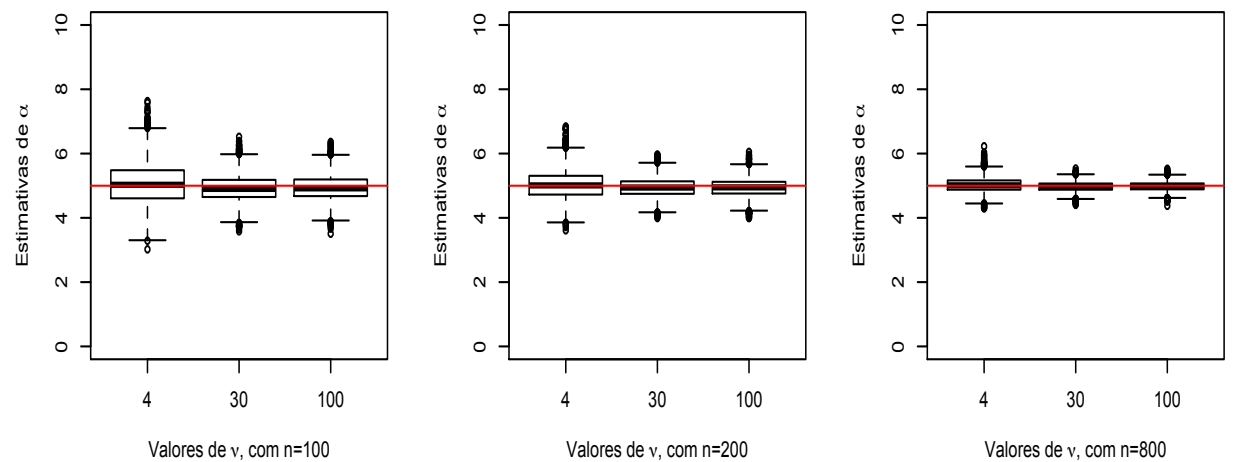

MV t-Student
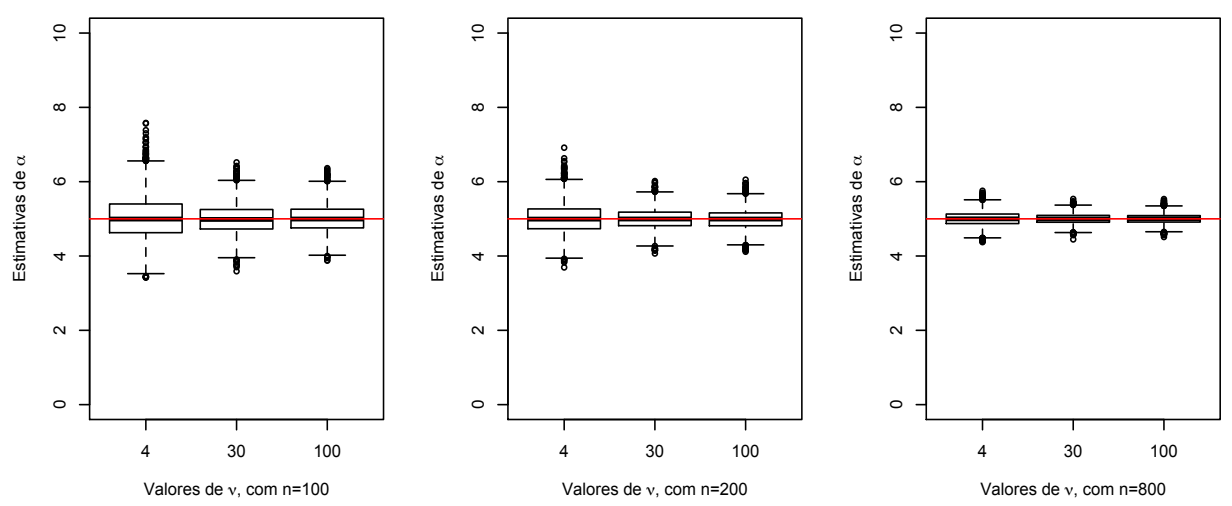

MVPM
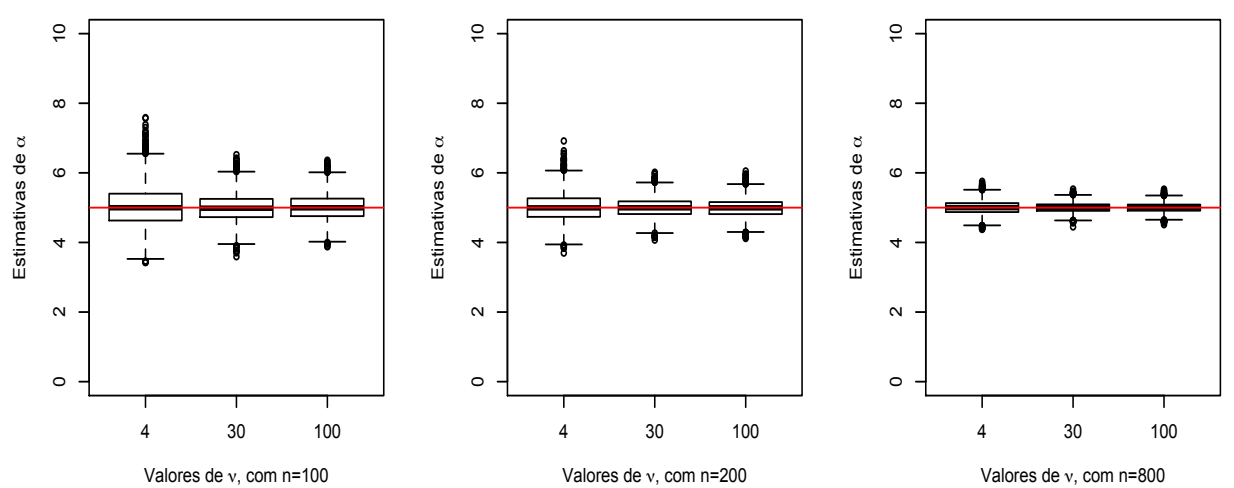

MVPMA

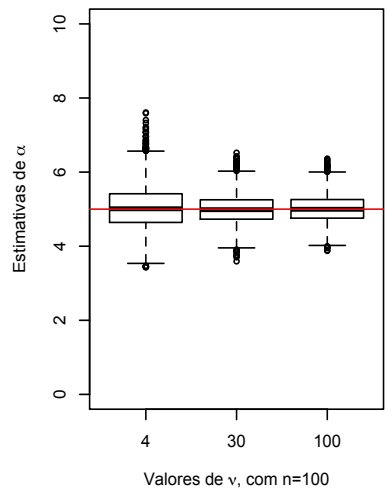

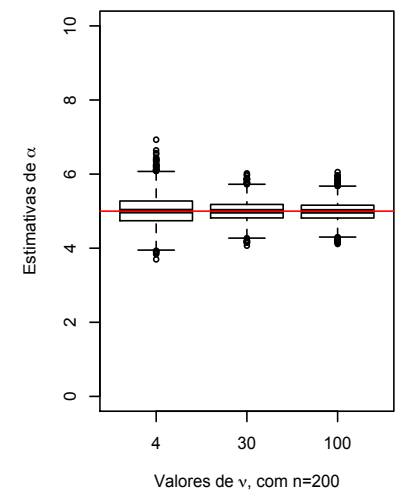

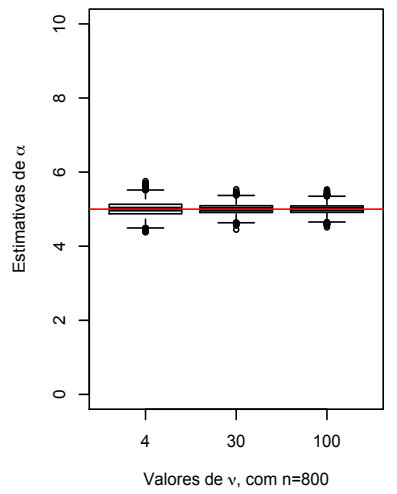

Figura 6.3: Gráficos boxplots para as estimativas de $\alpha$, considerado um modelo misto linear $t$-Student balanceado com valores dos parâmetros $\alpha=5$ e $\gamma=5$. 

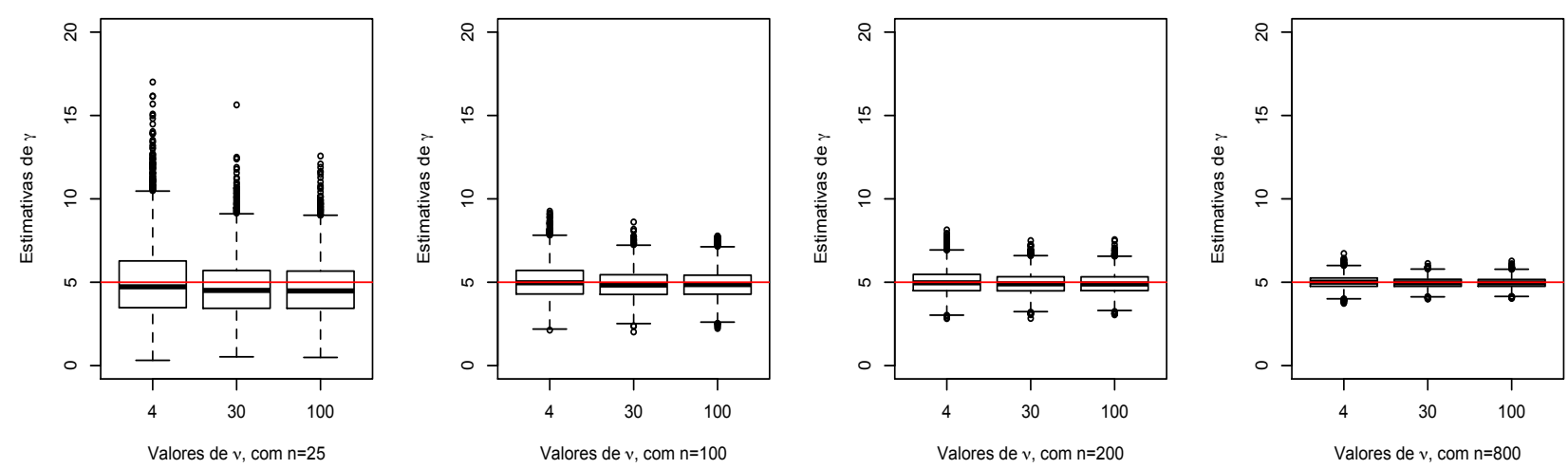

MV t-Student
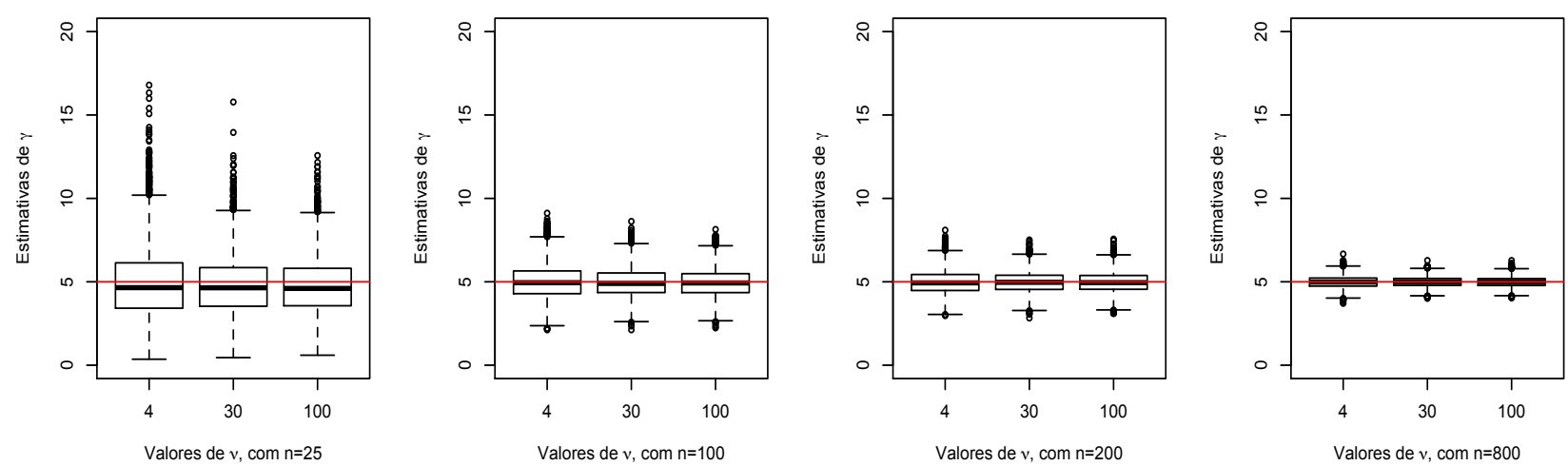

MVPM
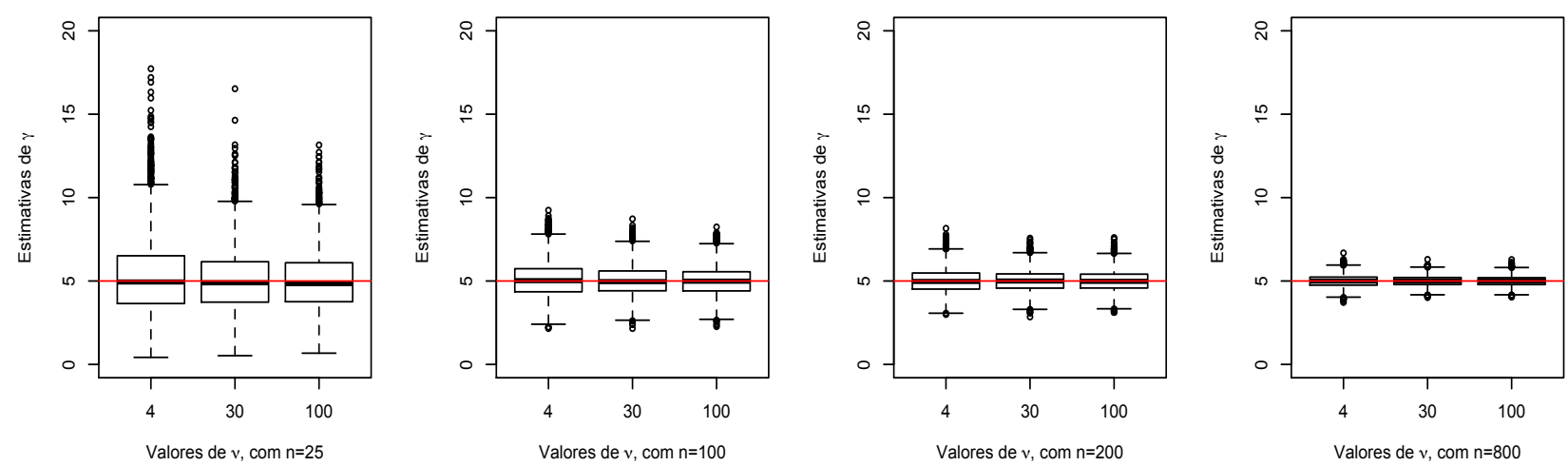

MVPMA
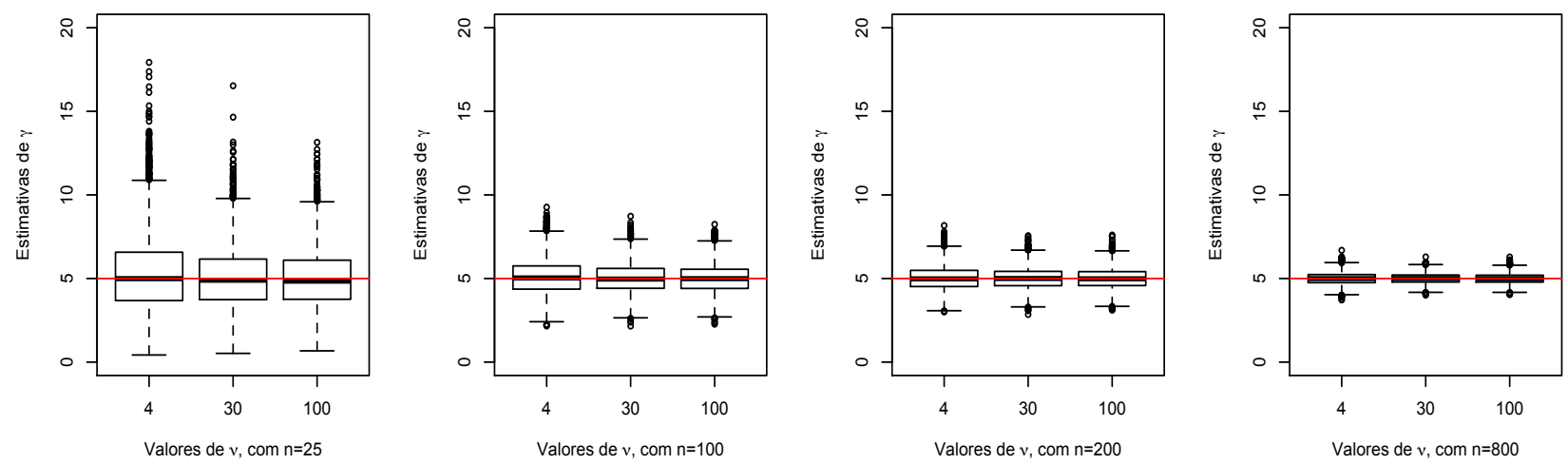

Figura 6.4: Gráficos boxplots para as estimativas de $\gamma$, considerado um modelo misto linear $t$-Student balanceado com valores dos parâmetros $\alpha=5$ e $\gamma=5$. 
Tabela 6.4: Estimativas de $\alpha$ e $\gamma$ obtidas pelos diferentes métodos de estimação, considerando o modelo misto linear t-Student balanceado ( $m=5)$ com valores de $\alpha=5$ e $\gamma=5$.

\begin{tabular}{|c|c|c|c|c|c|c|c|c|c|c|}
\hline \multirow{2}{*}{\multicolumn{2}{|c|}{$\alpha=5$}} & \multicolumn{3}{|c|}{$\nu=4$} & \multicolumn{3}{|c|}{$\nu=30$} & \multicolumn{3}{|c|}{$\nu=100$} \\
\hline & & MV & MVPM & MVPMA & MV & MVPM & MVPMA & MV & MVPM & MVPMA \\
\hline \multirow{3}{*}{$n=25$} & Média & 5.122 & 5.134 & 5.185 & 4.966 & 4.967 & 4.974 & 4.981 & 4.981 & 4.983 \\
\hline & Desvio padrão & 1.155 & 1.158 & 1.169 & 0.751 & 0.752 & 0.753 & 0.690 & 0.690 & 0.690 \\
\hline & Viés relativo & 0.024 & 0.027 & 0.037 & 0.007 & 0.007 & 0.005 & 0.004 & 0.004 & 0.003 \\
\hline \multirow{3}{*}{$n=100$} & Média & 5.006 & 5.008 & 5.021 & 4.984 & 4.984 & 4.986 & 5.002 & 5.002 & 5.002 \\
\hline & Desvio padrão & 0.544 & 0.544 & 0.546 & 0.391 & 0.391 & 0.391 & 0.368 & 0.368 & 0.368 \\
\hline & Viés relativo & 0.001 & 0.002 & 0.004 & 0.003 & 0.003 & 0.003 & 0.000 & 0.000 & 0.000 \\
\hline \multirow{3}{*}{$n=200$} & Média & 5.014 & 5.016 & 5.022 & 5.007 & 5.007 & 5.008 & 4.998 & 4.998 & 4.998 \\
\hline & Desvio padrão & 0.395 & 0.395 & 0.395 & 0.281 & 0.280 & 0.280 & 0.254 & 0.254 & 0.254 \\
\hline & Viés relativo & 0.003 & 0.003 & 0.004 & 0.001 & 0.001 & 0.002 & 0.000 & 0.000 & 0.000 \\
\hline \multirow{3}{*}{$n=800$} & Média & 5.006 & 5.007 & 5.008 & 5.000 & 5.000 & 5.000 & 5.003 & 5.003 & 5.003 \\
\hline & Desvio padrão & 0.188 & 0.188 & 0.188 & 0.141 & 0.141 & 0.141 & 0.126 & 0.126 & 0.126 \\
\hline & Viés relativo & 0.001 & 0.001 & 0.002 & 0.000 & 0.000 & 0.000 & 0.001 & 0.001 & 0.001 \\
\hline \multirow{2}{*}{\multicolumn{2}{|c|}{$\gamma=5$}} & \multicolumn{3}{|c|}{$\nu=4$} & \multicolumn{3}{|c|}{$\nu=30$} & \multicolumn{3}{|c|}{$\nu=100$} \\
\hline & & MV & MVPM & MVPMA & MV & MVPM & MVPMA & MV & MVPM & MVPMA \\
\hline \multirow{3}{*}{$n=25$} & Média & 5.009 & 5.333 & 5.380 & 4.867 & 5.126 & 5.131 & 4.719 & 4.962 & 4.964 \\
\hline & Desvio padrão & 2.087 & 2.202 & 2.221 & 1.842 & 1.923 & 1.924 & 1.692 & 1.764 & 1.765 \\
\hline & Viés relativo & 0.002 & 0.067 & 0.076 & 0.027 & 0.025 & 0.026 & 0.056 & 0.008 & 0.007 \\
\hline \multirow{3}{*}{$n=100$} & Média & 4.940 & 5.016 & 5.029 & 4.902 & 4.965 & 4.967 & 4.962 & 5.023 & 5.023 \\
\hline & Desvio padrão & 0.994 & 1.007 & 1.010 & 0.867 & 0.876 & 0.877 & 0.865 & 0.874 & 0.874 \\
\hline & Viés relativo & 0.012 & 0.003 & 0.006 & 0.020 & 0.007 & 0.007 & 0.008 & 0.005 & 0.005 \\
\hline \multirow{3}{*}{$n=200$} & Média & 4.967 & 5.005 & 5.011 & 4.959 & 4.991 & 4.991 & 4.953 & 4.984 & 4.984 \\
\hline & Desvio padrão & 0.703 & 0.708 & 0.709 & 0.639 & 0.642 & 0.642 & 0.596 & 0.599 & 0.599 \\
\hline & Viés relativo & 0.007 & 0.001 & 0.002 & 0.008 & 0.002 & 0.002 & 0.009 & 0.003 & 0.003 \\
\hline \multirow{3}{*}{$n=800$} & Média & 4.999 & 5.009 & 5.010 & 4.975 & 4.983 & 4.983 & 5.003 & 5.011 & 5.011 \\
\hline & Desvio padrão & 0.358 & 0.359 & 0.359 & 0.329 & 0.329 & 0.330 & 0.302 & 0.302 & 0.302 \\
\hline & Viés relativo & 0.000 & 0.002 & 0.002 & 0.005 & 0.003 & 0.003 & 0.001 & 0.002 & 0.002 \\
\hline
\end{tabular}




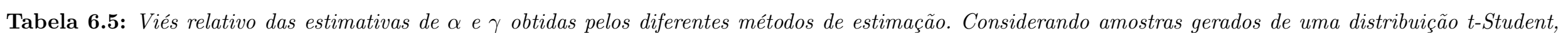
com $m$ de tamanhos 5,15 e 45 e valores de $\alpha=5$ e $\gamma=5$.

\begin{tabular}{|c|c|c|c|c|c|c|c|c|c|c|c|c|}
\hline \multirow{3}{*}{$\alpha=5$} & \multicolumn{4}{|c|}{$\mathrm{m}=5$} & \multicolumn{4}{|c|}{$\mathrm{m}=15$} & \multicolumn{4}{|c|}{$\mathrm{m}=45$} \\
\hline & \multicolumn{2}{|c|}{ t-Student } & \multicolumn{2}{|c|}{ Normal } & \multicolumn{2}{|c|}{ t-Student } & \multicolumn{2}{|c|}{ Normal } & \multicolumn{2}{|c|}{ t-Student } & \multicolumn{2}{|c|}{ Normal } \\
\hline & MV & MVPM & MV & MVPM & MV & MVPM & MV & MVPM & MV & MVPM & MV & MVPM \\
\hline$n=25$ & 0.024 & 0.027 & 1.001 & 1.001 & 0.025 & 0.026 & 1.011 & 1.011 & 0.028 & 0.029 & 1.088 & 1.088 \\
\hline$n=100$ & 0.001 & 0.002 & 0.991 & 0.991 & 0.005 & 0.005 & 0.999 & 0.999 & 0.008 & 0.008 & 0.999 & 0.999 \\
\hline$n=200$ & 0.003 & 0.003 & 0.999 & 0.999 & 0.003 & 0.003 & 1.006 & 1.006 & 0.002 & 0.002 & 1.011 & 1.011 \\
\hline$n=800$ & 0.001 & 0.001 & 0.999 & 0.999 & 0.001 & 0.001 & 0.996 & 0.996 & 0.000 & 0.000 & 0.999 & 0.999 \\
\hline \multirow{3}{*}{$\gamma=5$} & \multicolumn{4}{|c|}{$\mathrm{m}=5$} & \multicolumn{4}{|c|}{$\mathrm{m}=15$} & \multicolumn{4}{|c|}{$\mathrm{m}=45$} \\
\hline & \multicolumn{2}{|c|}{ t-Student } & \multicolumn{2}{|c|}{ Normal } & \multicolumn{2}{|c|}{ t-Student } & \multicolumn{2}{|c|}{ Normal } & \multicolumn{2}{|c|}{ t-Student } & \multicolumn{2}{|c|}{ Normal } \\
\hline & MV & MVPM & MV & MVPM & MV & MVPM & MV & MVPM & MV & MVPM & MV & MVPM \\
\hline$n=25$ & 0.002 & 0.067 & 0.867 & 0.961 & 0.018 & 0.025 & 0.923 & 1.005 & 0.018 & 0.025 & 0.923 & 1.006 \\
\hline$n=100$ & 0.012 & 0.003 & 0.983 & 1.007 & 0.006 & 0.006 & 0.983 & 1.003 & 0.005 & 0.006 & 0.983 & 1.003 \\
\hline$n=200$ & 0.007 & 0.001 & 0.975 & 0.987 & 0.003 & 0.003 & 0.982 & 0.992 & 0.003 & 0.004 & 0.982 & 0.992 \\
\hline$n=800$ & 0.000 & 0.002 & 0.989 & 0.992 & 0.000 & 0.001 & 0.995 & 0.998 & 0.001 & 0.001 & 0.995 & 0.998 \\
\hline
\end{tabular}

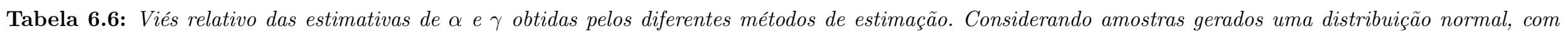
$m$ (grupos) de tamanhos 5, 15 e 45, tamanhos de amostra 25, 100, 200 e 800, para valores de $\alpha=5$ e $\gamma=5$.

\begin{tabular}{|c|c|c|c|c|c|c|c|c|c|c|c|c|}
\hline \multirow{3}{*}{$\alpha=5$} & \multicolumn{4}{|c|}{$m=5$} & \multicolumn{4}{|c|}{$\mathrm{m}=15$} & \multicolumn{4}{|c|}{$\mathrm{m}=45$} \\
\hline & \multicolumn{2}{|c|}{ t-Student } & \multicolumn{2}{|c|}{ Normal } & \multicolumn{2}{|c|}{ t-Student } & \multicolumn{2}{|c|}{ Normal } & \multicolumn{2}{|c|}{ t-Student } & \multicolumn{2}{|c|}{ Normal } \\
\hline & MV & MVPM & MV & MVPM & MV & MVPM & MV & MVPM & MV & MVPM & MV & MVPM \\
\hline$n=25$ & 0.195 & 0.195 & 0.001 & 0.001 & 0.102 & 0.102 & 0.002 & 0.002 & 0.040 & 0.040 & 0.001 & 0.001 \\
\hline$n=100$ & 0.198 & 0.198 & 0.001 & 0.001 & 0.101 & 0.101 & 0.001 & 0.001 & 0.040 & 0.040 & 0.000 & 0.000 \\
\hline$n=200$ & 0.200 & 0.200 & 0.000 & 0.000 & 0.101 & 0.101 & 0.001 & 0.001 & 0.041 & 0.041 & 0.000 & 0.000 \\
\hline$n=800$ & 0.200 & 0.200 & 0.000 & 0.000 & 0.103 & 0.103 & 0.000 & 0.000 & 0.041 & 0.041 & 0.000 & 0.000 \\
\hline \multirow{3}{*}{$\gamma=5$} & \multicolumn{4}{|c|}{$\mathrm{m}=5$} & \multicolumn{4}{|c|}{$\mathrm{m}=15$} & \multicolumn{4}{|c|}{$\mathrm{m}=45$} \\
\hline & \multicolumn{2}{|c|}{ t-Student } & \multicolumn{2}{|c|}{ Normal } & \multicolumn{2}{|c|}{ t-Student } & \multicolumn{2}{|c|}{ Normal } & \multicolumn{2}{|c|}{ t-Student } & \multicolumn{2}{|c|}{ Normal } \\
\hline & MV & MVPM & MV & MVPM & MV & MVPM & MV & MVPM & MV & MVPM & MV & MVPM \\
\hline$n=25$ & 0.235 & 0.191 & 0.053 & 0.006 & 0.140 & 0.097 & 0.048 & 0.005 & 0.081 & 0.040 & 0.046 & 0.006 \\
\hline$n=100$ & 0.207 & 0.195 & 0.011 & 0.001 & 0.110 & 0.099 & 0.011 & 0.001 & 0.049 & 0.038 & 0.009 & 0.001 \\
\hline$n=200$ & 0.203 & 0.197 & 0.006 & 0.000 & 0.106 & 0.101 & 0.003 & 0.003 & 0.045 & 0.040 & 0.005 & 0.000 \\
\hline$n=800$ & 0.201 & 0.200 & 0.002 & 0.000 & 0.103 & 0.102 & 0.000 & 0.002 & 0.043 & 0.042 & 0.003 & 0.002 \\
\hline
\end{tabular}




\subsection{Aplicações}

Nesta seção consideraremos duas aplicações associadas a dois modelos considerados nos cenários de simulação da seção anterior.

\subsubsection{Volume do Plasma}

Os modelos associados à comparação de medições têm sido muito utilizados nos estudos clínicos devido à importância de comparar as medições feitas por dois ou mais instrumentos e avaliar a concordância de suas medições. Os dados utilizados nesta seção correspondem ao volume de plasma de 99 pacientes medidos em porcentagem utilizando dois métodos de medição: Hurley e Nadler. Originalmente, foram fornecidos por Cotes et al. (1986) num estudo clínico e posteriormente reportado por Martin e Altman (1999) na análise de uma medida de concordância em comparação de instrumentos de medição. Estes autores apresentam gráficos para analisar a concordância das medidas dos instrumentos. Choudhary e Nagaraja (2017) discutem os gráficos e as análises propostas por Martin e Altman (1999) e ajustam um modelo misto linear. Os dados podem ser obtidos da biblioteca MethComp de Carstensen et al. (2015) do software R.

\section{Descrição dos dados}

Na Figura 6.5 tem-se a relação entre as medições. Se as medições dos métodos forem idênticas, no gráfico (a) esperamos todos os pontos sobre a reta na diagonal. Mas como podemos notar pelo gráfico (a) medições do método Nalder são maiores do que as medições do método Hurley. Ainda mais, como a nuvem de pontos não é exatamente paralela à reta, as medições do método Nalder parecem não ser proporcionais às medições do método Hurley. No gráfico (b) representa-se as diferenças das medições dos métodos para cada paciente. Vemos mais claramente que são diferentes e dada a nuvem de pontos podemos supor uma tendência linear entre as medidas. Nos gráficos (c) e (d) consideramos a transformação do logaritmo das medições dos métodos, este tipo de transformação é utilizada para remover uma possível heteroscedasticidade.

\section{Modelo proposto}

Vamos denotar o vetor do logaritmo das medições do método Hurley por $\mathbf{Y}_{1}$ e do método Nalder como $\mathbf{Y}_{2}$. O modelo usualmente proposto para estudar a relação entre as medições dos métodos é um modelo linear. Considerando o $i$-ésimo paciente, com $i=1, \ldots, 99$, o modelo fica dado por

$$
\begin{aligned}
& Y_{i 1}=s_{i}+\epsilon_{i 1} \\
& Y_{i 2}=\beta_{0}+s_{i}+\epsilon_{i 2}
\end{aligned}
$$

em que $s_{i}$ é uma variável aleatória que representa o verdadeiro volume do plasma do $i$-ésimo paciente mas que não pode ser medido de forma exata. Enquanto $\epsilon_{i j}$, com $j=1,2$, representa o erro de medição do $j$-ésimo método associado ao $i$-ésimo paciente. O método Hurley foi assumido como o método de referência, portanto as medições do método Nalder são representadas da mesma forma que o método Hurley mais uma constante $\beta_{0}$ (viés aditivo). Então, uma forma de comparar as medições é observando o valor de $\beta_{0}$, se tende a zero podemos pensar que as medições dos métodos são similares. Como vimos nos gráficos (a) e (c) da Figura 6.5 as medições do método Nalder são maiores do que as medições do método Hurley, portanto esperamos que a estimativa de $\beta_{0}$ seja positiva. Assumindo que variável aleatória $s_{i}$ tem média $\mu_{s}$ e variância $\gamma$, vamos considerar $d_{i}$ uma variável aleatória que representa a transformação $s_{i}-\mu_{s}$, com média zero e variância $\gamma$. Assim, $Y_{i 1}$ pode ser escrita como: $\mu_{s}+d_{i}+\epsilon_{i 1}$ e $Y_{i 2}$ como $\beta_{0}+\mu_{s}+d_{i}+\epsilon_{i 2}$. Para os erros $\epsilon_{i 1}$ e $\epsilon_{i 2}$ vamos supor que ambos tem média 0 e variância $\alpha$.

Para escrever o modelo misto linear t-Student, consideramos que as medições do volume do plasma de ambos os métodos correspondem ao mesmo paciente e temos que o $i$-ésimo vetor de 


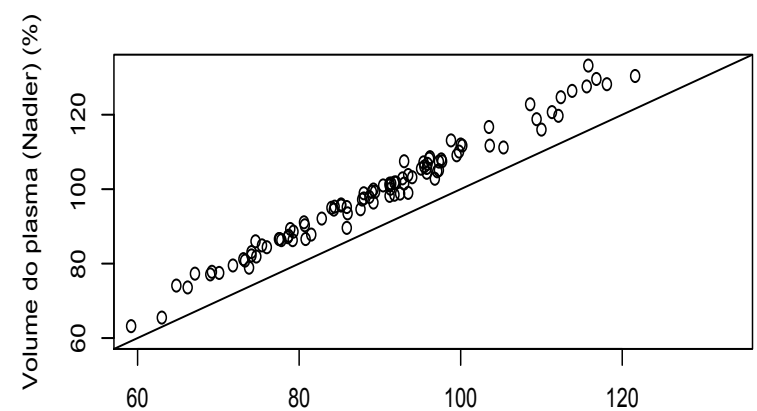

(a) Volume do plasma (Hurley) (\%)

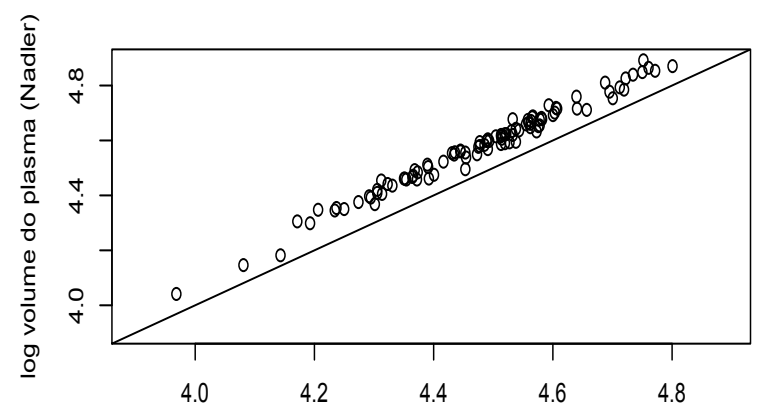

(c) log volume do plasma (Hurley)

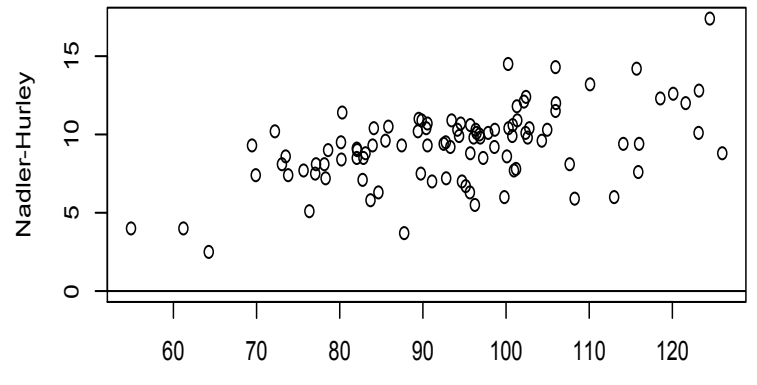

(b) Média entre Nadler e Hurley

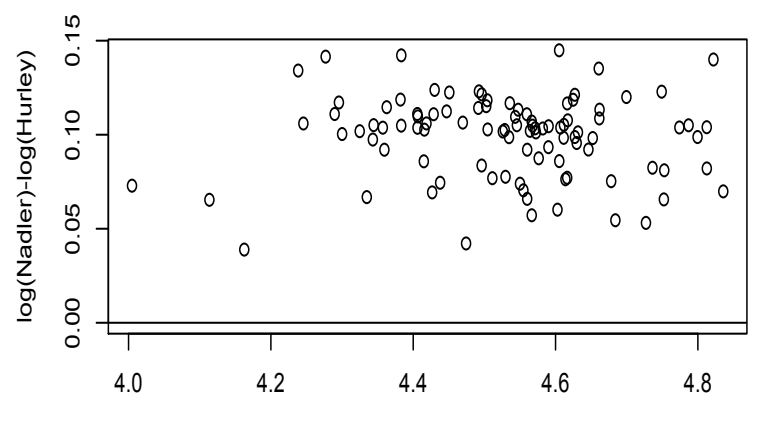

(d) Média entre log(Nadler) e log(Hurley)

Figura 6.5: Gráficos de comparação do volume do plasma obtidos pelos métodos Hurley e Nalder.

respostas é dado por $\mathbf{Y}_{i}=\left(Y_{i 1}, Y_{i 2}\right)^{\top}$ de dimensão $2 \times 1$, para $i=1, \ldots, 99$, e o modelo pode ser escrito como:

$$
\mathbf{Y}_{i}=\mathbf{I}_{2} \boldsymbol{\beta}+d_{i} \mathbf{1}_{2}+\boldsymbol{\epsilon}_{i},
$$

em que $\boldsymbol{\beta}$ é um vetor $(2 \times 1)$ com elementos $\beta_{1}=\mu_{s}$ e $\beta_{2}=\mu_{s}+\beta_{0}$. Assim, a interpretação de $\beta_{0}$ continua tendo o mesmo sentido. Supondo que o vetor $\left(d_{i}, \boldsymbol{\epsilon}_{i}^{\top}\right)^{\top}$ tem distribuição t-Student com média igual $\mathbf{0}_{3}$ e matriz de escala dada por

$$
\left(\begin{array}{cc}
\gamma & \mathbf{0}^{\top} \\
\mathbf{0} & \alpha \mathbf{I}_{2}
\end{array}\right)
$$

e $\nu$ graus de liberdade. Então, temos que $\mathbf{Y}_{i} \sim t_{2}(\boldsymbol{\beta}, \boldsymbol{\Sigma}, \nu)$, com $\boldsymbol{\Sigma}=\alpha \mathbf{I}_{2}+\gamma \mathbf{J}_{2}$, para $i=1, \ldots, 99$. Dado que os graus de liberdade são considerados fixos, vamos escolher os graus de liberdade que tenham menor critério de informação de Akaike (1974)(AIC). Na Tabela 6.7 vemos que o menor AIC é obtido considerando 5 graus de liberdade.

Tabela 6.7: Escolha dos graus de liberdade.

\begin{tabular}{|c|c|c|c|c|c|c|}
\hline$\nu$ & 3 & 4 & 5 & 6 & 7 & 8 \\
\hline AIC & 280.791 & 532.743 & $\mathbf{2 6 0 . 2 5 5}$ & 288.842 & 290.234 & 291.428 \\
\hline
\end{tabular}

Na Tabela 6.8 apresentamos as estimativas de máxima verossimilhança (MV) e máxima verossimilhança restrita (MVR) considerando um modelo misto linear normal. Para o modelo misto linear t-Student temos as estimativas obtidas pelos métodos de máxima verossimilhança (MV), máxima verossimilhança perfilada modificada (MVPM) e máxima verossimilhança perfilada modificada apróximada (MVPMA). Dado que apenas temos duas covariáveis e 99 dados, vemos que as 
estimativas de MVR não tem discrepância com respeito às estimativas de MV no modelo normal. No modelo t-Student vemos que as estimativas obtidas pelos diferentes métodos são similares. O mesmo ocorre quando comparamos as estimativas sob erros normais e erros t-Student. Em conclusão, o logaritmo das medições do método de Nalder em média excedem em 0.10 o logaritmo das medições do método de Hurley.

Tabela 6.8: Resultados das estimativas considerando o modelo normal e o modelo t-Student. ${ }^{a}$

\begin{tabular}{|l|rr|rrr|}
\cline { 2 - 6 } \multicolumn{1}{c|}{} & \multicolumn{2}{|c|}{ Normal } & \multicolumn{3}{c|}{ t-Student, com $\nu=5$} \\
\cline { 2 - 7 } & MV & MVR & MV & MVPM & MVPMA \\
\hline$\beta_{0}$ & 0.0989 & 0.0989 & 0.1009 & 0.1009 & 0.1008 \\
$\beta_{1}$ & 4.4789 & 4.4789 & 4.4852 & 4.4851 & 4.4851 \\
$\alpha$ & 0.0002 & 0.0002 & 0.0002 & 0.0002 & 0.0002 \\
$\gamma$ & 0.0250 & 0.0253 & 0.0171 & 0.0173 & 0.0174 \\
\hline
\end{tabular}

Para avaliar o ajuste dos modelos normal e t-Student, utilizaremos um gráfico da distância transformadas (ver Little (1988), Lange et al. (1989)). De forma geral, denotamos a distância de Mahalanobis, $\left(\mathbf{Y}_{i}-\mathbf{X}_{i} \boldsymbol{\beta}\right)^{\top} \boldsymbol{\Sigma}_{i}^{-1}\left(\mathbf{Y}_{i}-\mathbf{X}_{i} \boldsymbol{\beta}\right)$, por $\delta_{i}$ e a distância avaliada nos estimadores de máxima verossimilhança dos parâmetros desconhecidos por $\hat{\delta}_{i}$, para $i=1, \ldots, n$. Além disso, consideramos a propriedade das distribuições elípticas dada na equação (C.5) da Seção C.2 do Apêndice C.

Para o modelo normal temos que $\delta_{i}$ tem distribuição $\chi^{2}$ com $m_{i}$ graus de liberdade, para $i=1, \ldots, n$. Por propriedade dos estimadores de máxima verossimilhança num modelo normal temos que a distribuição assintótica de $\hat{\delta}_{i}$ tem a mesma distribuição $\chi^{2} \operatorname{com} m_{i}$ graus de liberdade, para $i=1, \ldots, n$. Assim, da transformação de Wilson-Hilferty (ver Johnson et al. (1994)) temos que a distância transforma fica dada por

$$
d_{i}=\frac{\left(\frac{\hat{\delta}_{i}}{m_{i}}\right)^{1 / 3}-1+\left(\frac{2}{9 m_{i}}\right)}{\left(\frac{2}{9 m_{i}}\right)^{1 / 2}}
$$

em que $d_{i}$ tem aproximadamente uma distribuição normal padrão, para $i=1, \ldots, n$.

Para o modelo t-Student, temos que $\delta_{i} / m_{i}$ tem distribuição $F$ com $m_{i}$ e $\nu$ graus de liberdade, para $i=1, \ldots, n$. A distribuição assintótica de $\hat{\delta}_{i}$ também tem distribuição $\mathrm{F}$ com $m_{i}$ e $\nu$ graus de liberdade, para $i=1, \ldots, n$. Da transformação de Wilson-Hilferty (ver Johnson et al. (1994)) temos que a distância transformada do modelo t-Student fica dada por

$$
d t_{i}=\frac{\left(1-\frac{2}{9 \nu}\right)\left(\frac{\hat{\delta}_{i}}{m_{i}}\right)^{1 / 3}-1+\frac{2}{9 m_{i}}}{\left\{\frac{2}{9 \nu}\left(\frac{\hat{\delta}_{i}}{m_{i}}\right)^{2 / 3}+\frac{2}{9 m_{i}}\right\}^{1 / 2}}
$$

em que $d t_{i}$ tem aproximadamente uma distribuição normal padrão, para $i=1, \ldots, n$.

Para os dados do volume de plasma, temos que $m_{i}=2$, para $i=1, \ldots, 99$ e $\nu=5$. Na Figura 6.6 comparamos os ajustes das distâncias transformadas dos modelos normal e t-Student com respeito aos quantis de uma distribuição normal padrão. Vemos que o ajuste b) das distâncias transformadas do modelo t-Student tem uma maior quantidade de pontos sobre a reta, portanto apresenta um ajuste mais adequado que o modelo normal.

Mediante o procedimento bootstrap não paramétrico na Tabela 6.9 apresentamos um resumo das estimativas obtidas da reamostragem de 1000 amostras. Também, especificamos os desvios padrão que podem ser considerados uma aproximação aos erros padrão das estimativas. Vemos que, em geral, os desvios padrão das estimativas de MVPM e MVPMA são menores à excepção do parâmetro $\gamma$ 
a)

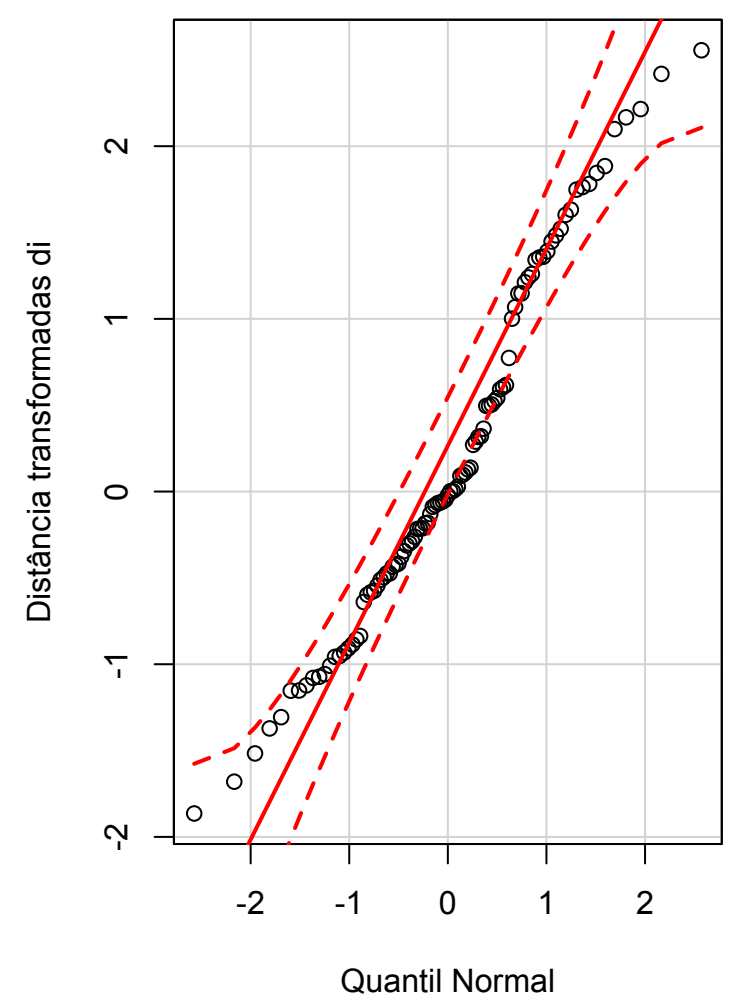

b)

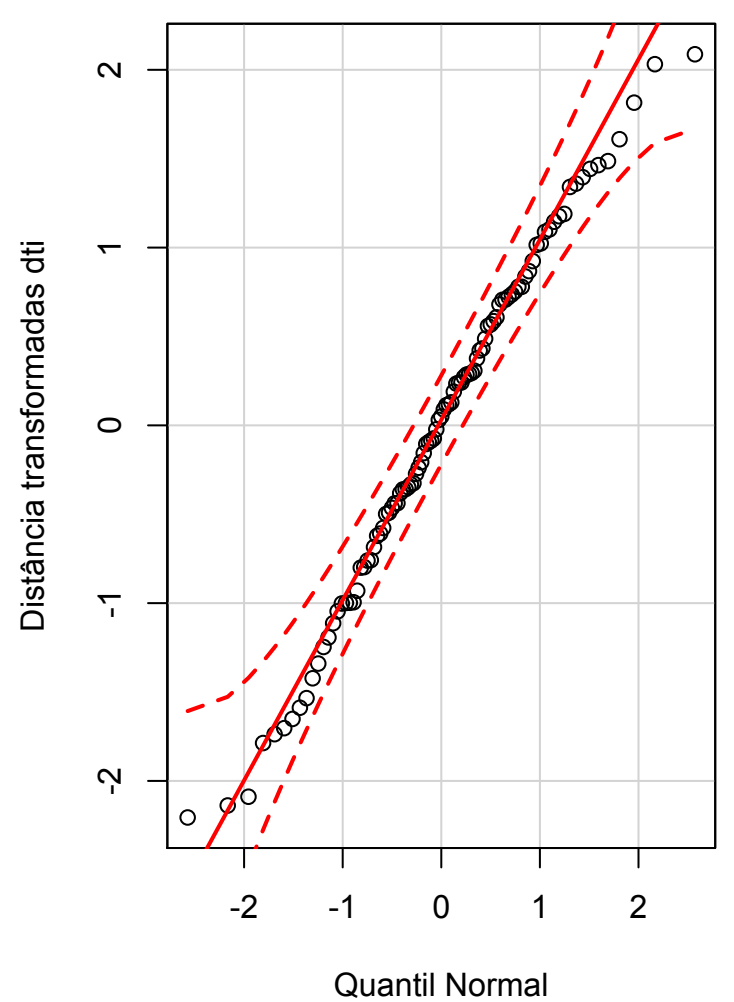

Figura 6.6: qqplots das distâncias transformadas, a) modelo normal e b) modelo t-Student.

Tabela 6.9: Estimativas bootstrap dos parâmetros para o modelo normal e modelo t-Student, obtidas pelos diferentes métodos de estimação.

\begin{tabular}{|ll|cc|ccr|}
\cline { 3 - 7 } \multicolumn{1}{c|}{} & \multicolumn{2}{c|}{ Normal } & \multicolumn{3}{c|}{ t-Student, com $\nu=5$} \\
\cline { 3 - 7 } \multicolumn{2}{c|}{} & MV & MVR & MV & MVPM & MVPMA \\
\hline \multirow{2}{*}{$\beta_{0}$} & Média & 0.01192 & 0.01193 & 0.10020 & 0.10138 & 0.10137 \\
& Desvio padrão & 0.00696 & 0.00697 & 0.02391 & 0.00211 & 0.00211 \\
\hline \multirow{2}{*}{$\beta_{1}$} & Média & 4.51882 & 4.51882 & 4.64597 & 4.58308 & 4.58306 \\
& Desvio padrão & 0.01547 & 0.01548 & 0.15891 & 0.01572 & 0.01572 \\
\hline \multirow{2}{*}{$\alpha$} & Média & 0.02782 & 0.02807 & 0.00153 & 0.00015 & 0.00016 \\
& Desvio padrão & 0.00426 & 0.00430 & 0.03046 & 0.00005 & 0.00005 \\
\hline \multirow{2}{*}{$\gamma$} & Média & 0.00020 & 0.00024 & 0.24525 & 0.01743 & 0.01756 \\
& Desvio padrão & 0.00057 & 0.00062 & 0.50759 & 0.00305 & 0.00307 \\
\hline
\end{tabular}

\subsubsection{Dados censitários de Boston}

No estudo realizado por Harrison e Rubinfeld (1978) foi proposto um modelo hedônico do preço da habitação para medir a disposição de pagar por ar limpo. Para isso, utilizam uma amostra de setores censitários de Boston Standard Metropolitan Statistical Area, em 1970. Os autores utilizam como variável dependente o logaritmo do valor mediano das casas ocupadas pelos proprietários no setor censitário considerando um modelo de efeitos fixos. Belsley et al. (1980) fazem uma análise dos dados propondo um modelo de regressão linear multipla. Enquanto que Zhong et al. (2002) propõem um modelo misto linear para um subconjunto dos dados. Na Tese de Doutorado de Borssoi (2013) e no artigo de Borssoi et al. (2017) é utilizado o mesmo subconjunto de dados de Zhong et al. (2002), porém num modelo misto linear elíptico com uma variável explicativa sujeita a erros de medição. 
Tabela 6.10: Descrição das variáveis dados censitários de Boston.

\begin{tabular}{l|l} 
Variável & Descrição da variável \\
\hline LMV & $\begin{array}{l}\text { Logaritmo do valor mediano das casas ocupadas pelos proprietários } \\
\text { em USD } \\
\text { Taxa de criminalidade por cidade }\end{array}$ \\
CRIME & $\begin{array}{l}\text { Variável dummy do Rio Charles com valor 1 se limites das vias sobre } \\
\text { o rio e 0 caso contrário }\end{array}$ \\
NOXSQ & $\begin{array}{l}\text { Concentração de óxido de nitrogênio (partes por 100 milhões) ao quadrado } \\
\text { ROÓmero médio de quartos ao quadrado }\end{array}$ \\
AGE & $\begin{array}{l}\text { Proporção de unidades ocupadas pelos proprietários construídas } \\
\text { antes de } 1940\end{array}$ \\
DIST & $\begin{array}{l}\text { Logaritmo das distâncias ponderadas para cinco centros de emprego da } \\
\text { região de Boston } \\
(B k-0.63)^{2} \text { em que } B k \text { é a proporção de negros na população }\end{array}$ \\
BLACK & Logaritmo da proporção da população com baixa renda \\
LSTAT
\end{tabular}

Neste caso consideramos o subconjunto de dados utilizados por estes últimos autores.

Os dados originais e a descrição das variáveis podem ser obtido do software $\mathrm{R}$ na biblioteca $\mathrm{ml}$ bench de Leisch e Dimitriadou (2010). O subconjunto de dados utilizados por Zhong et al. (2002) e que analisaremos nesta seção, está também disponível na Tese de Doutorado de Borssoi (2013). Consideramos um modelo misto linear t-Student.

\section{Descrição dos dados}

Os dados correspondem a 132 setores censitários dentro de 15 distritos de Boston obtidos em 1970. Na Tabela 6.10 apresentamos a descrição das variáveis que consideraremos nesta análise.

$\mathrm{Na}$ Tabela 6.11 apresentamos algumas estatísticas descritivas da variável LMV dos 15 distritos considerados. Observamos que os distritos considerados estão codificados do número 75 até o 89. O valor $m_{i}$, com $i=1, \ldots, 15$, representa a quantidade de setores para cada distrito. O distrito que contém a maior quantidade de setores é o distrito 84. Entanto que os distritos 77 e 78 são os que contêm informação apenas de três e dois setores, respectivamente. Além disso, a informação contida dentro desses distritos é a mesma para todos seus setores. Por outro lado, o distrito 81 tem a menor média (9.055) mas tem o maior coeficiente de variabilidade (CV(\%) 4.17). Levando em conta que a variável LMV é o logaritmo da variável original, observamos que os coeficientes de variabilidade dos distritos não são muito diferentes. Contudo, no primeiro gráfico da Figura 6.7, boxplot da variável LMV com respeito a cada distrito, observamos diferenças entre seus valores medianos.

Da mesma forma que foi apresentado na Tese de Doutorado de Borssoi (2013), as Figuras 6.7 e 6.8 apresentam os boxplots de cada covariável segundo cada distrito. Vemos que há diferenças entre os valores medianos dos distritos. Porém, para as covariáveis AGE e BLACK observamos que entre os distritos 75 e 81 não percebemos diferenças. Em relação aos pontos discrepantes vemos que a covariável CRIME é aquela que apresenta maior quantidade de pontos discrepantes. Nas Figuras 6.9 e 6.10 apresentamos os gráficos de dispersão de cada covariável com respeito à variável dependente LMV. Vemos, novamente, que a covariável CRIME apresenta pontos discrepantes da nuvem de pontos. O mesmo efeito que foi visto nos gráficos de dispersão das covariáveis ROOM e NOXSQ. Nos gráficos de dispersão das covariáveis AGE, DIST e BLACK não é clara a tendência linear, observamos uma mudança na variabilidade da variável dependente LMV à medida que as covariáveis aumentam. Contudo, na seguinte seção analisaremos um modelo misto linear t-Student considerando todas as covariáveis descritas nesta seção. Também, vamos comparar os resultados considerando um modelo misto linear. 
Tabela 6.11: Estatísticas descritivas da variável dependente LMV para os 15 distritos considerados.

\begin{tabular}{|c|r|rrrrrrrr|}
\hline Distrito & $m_{i}$ & Média & DP & CV(\%) & Min & Q1 & Med & Q3 & Max \\
\hline 75 & 8 & 9.941 & 0.130 & 1.380 & 9.729 & 9.871 & 9.964 & 10.030 & 10.130 \\
76 & 6 & 10.320 & 0.399 & 3.865 & 9.994 & 10.010 & 10.130 & 10.670 & 10.820 \\
77 & 3 & 10.820 & 0.000 & 0.000 & 10.820 & 10.820 & 10.820 & 10.820 & 10.820 \\
78 & 2 & 9.532 & 0.000 & 0.000 & 9.532 & 9.532 & 9.532 & 9.532 & 9.532 \\
79 & 7 & 9.415 & 0.154 & 1.635 & 9.230 & 9.273 & 9.480 & 9.518 & 9.616 \\
80 & 11 & 9.302 & 0.329 & 3.537 & 8.882 & 9.131 & 9.259 & 9.384 & 10.050 \\
81 & 13 & 9.055 & 0.378 & 4.170 & 8.517 & 8.748 & 9.048 & 9.433 & 9.532 \\
82 & 8 & 9.807 & 0.290 & 2.959 & 9.384 & 9.678 & 9.753 & 9.900 & 10.240 \\
83 & 19 & 9.314 & 0.310 & 3.331 & 8.854 & 9.059 & 9.297 & 9.557 & 9.943 \\
84 & 23 & 9.485 & 0.213 & 2.244 & 9.036 & 9.372 & 9.503 & 9.619 & 9.820 \\
85 & 11 & 9.770 & 0.177 & 1.814 & 9.449 & 9.657 & 9.852 & 9.901 & 9.971 \\
86 & 6 & 9.910 & 0.073 & 0.735 & 9.857 & 9.864 & 9.891 & 9.906 & 10.050 \\
87 & 7 & 9.715 & 0.319 & 3.287 & 9.393 & 9.514 & 9.589 & 9.847 & 10.300 \\
88 & 4 & 10.060 & 0.057 & 0.569 & 9.990 & 10.030 & 10.060 & 10.090 & 10.130 \\
89 & 4 & 9.921 & 0.045 & 0.450 & 9.857 & 9.914 & 9.933 & 9.940 & 9.962 \\
\hline
\end{tabular}
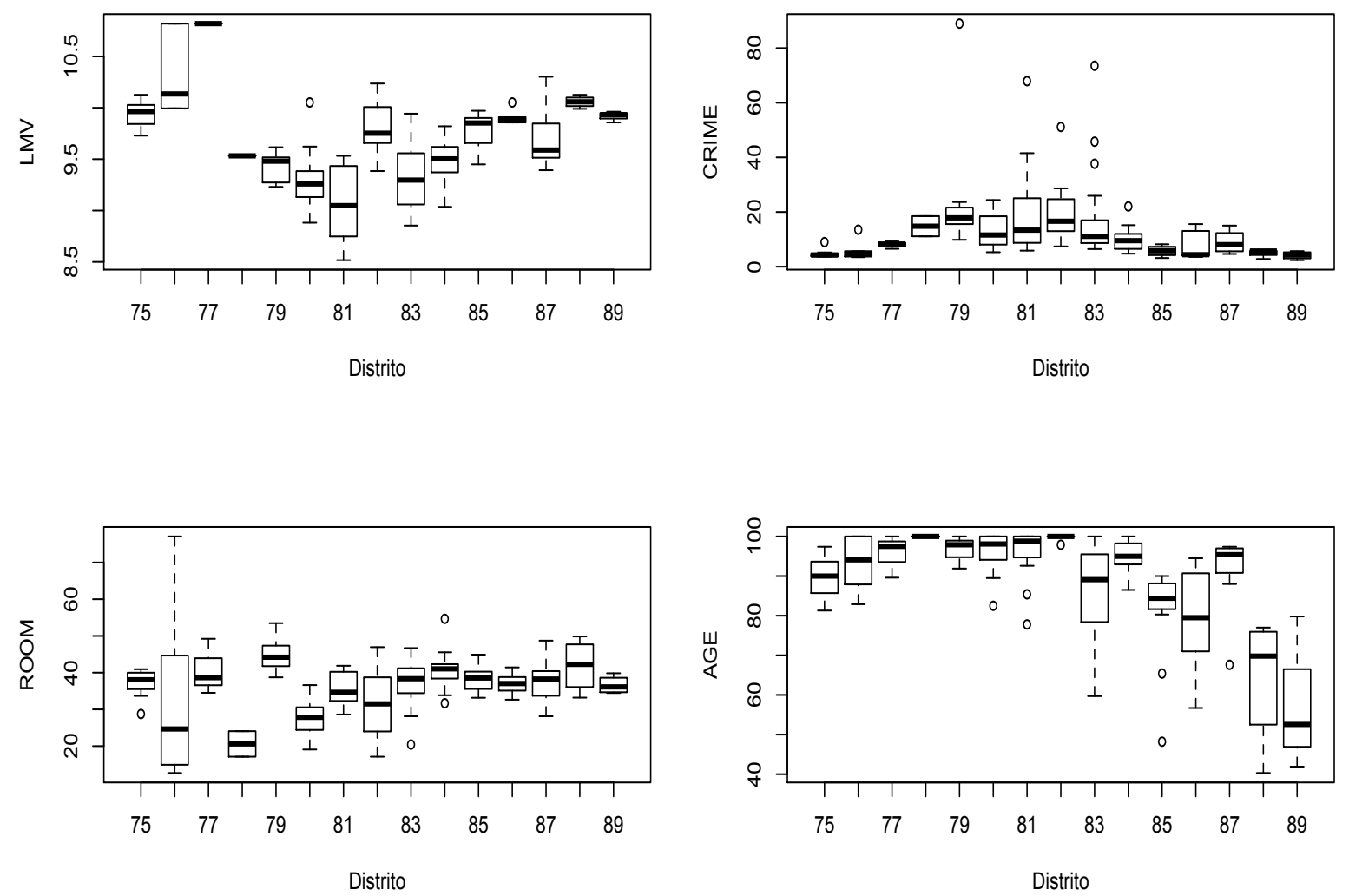

Figura 6.7: Gráficos boxplots das variáveis LMV, CRIME, ROOM e AGE com respeito aos distritos.

\section{Modelo proposto}

O modelo proposto é um modelo misto linear t-Student não balanceado, temos que $\mathbf{Y}_{i}$ é o vetor de respostas que representa a variável LMV, descrita na seção anterior, associada ao $i$-ésimo distrito, com $i=1, \ldots, 15$. A dimensão de cada $\mathbf{Y}_{i}$ é determinada pela quantidade de setores contidos em 


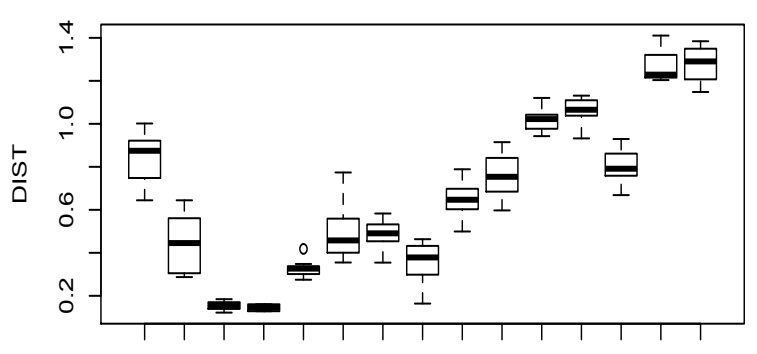

$\begin{array}{lllllllllllllll}75 & 76 & 77 & 78 & 79 & 80 & 81 & 82 & 83 & 84 & 85 & 86 & 87 & 88 & 89\end{array}$

Distrito

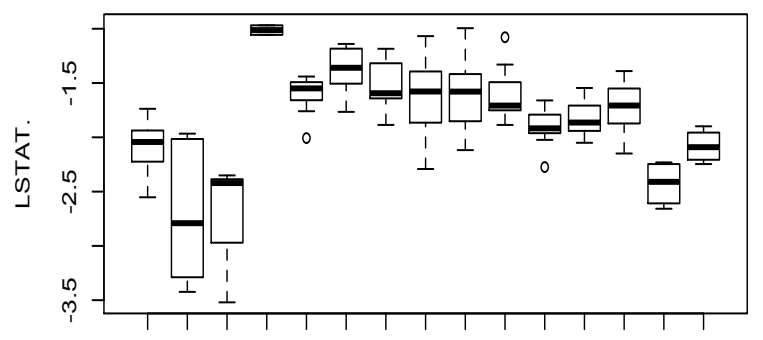

$\begin{array}{lllllllllllllll}75 & 76 & 77 & 78 & 79 & 80 & 81 & 82 & 83 & 84 & 85 & 86 & 87 & 88 & 89\end{array}$

Distrito

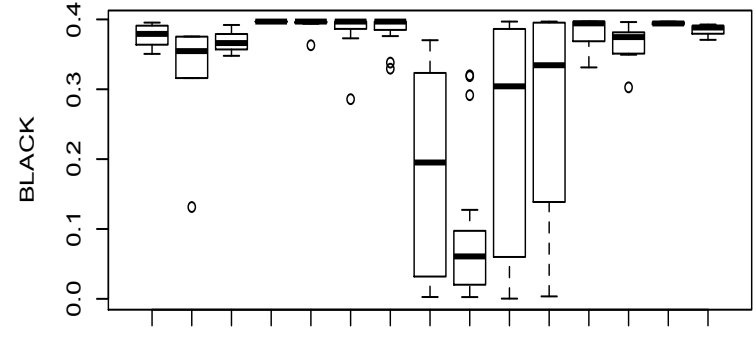

$\begin{array}{lllllllllllllll}75 & 76 & 77 & 78 & 79 & 80 & 81 & 82 & 83 & 84 & 85 & 86 & 87 & 88 & 89\end{array}$

Distrito

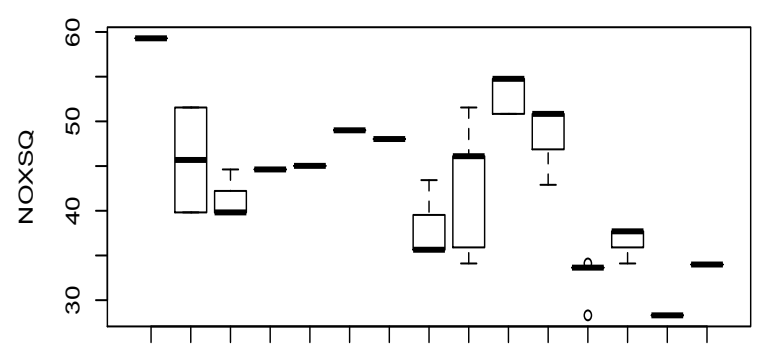

$\begin{array}{lllllllllllllll}75 & 76 & 77 & 78 & 79 & 80 & 81 & 82 & 83 & 84 & 85 & 86 & 87 & 88 & 89\end{array}$

Distrito

Figura 6.8: Gráficos boxplots das variáveis DIST, BLACK, LSTAT e NOXSQ, com respeito aos distritos.

cada distrito. Assim, para cada distrito podemos escrever o modelo da seguinte forma:

$$
\mathbf{Y}_{i}=\mathbf{X}_{i} \boldsymbol{\beta}+\mathbf{Z}_{i} d_{i}+\boldsymbol{\epsilon}_{i}, \operatorname{com} i=1, \ldots, 15
$$

em que $\mathbf{X}_{i}$ é uma matriz $m_{i} \times 9$ que contém um vetor de uns e as 8 covariáveis descritas na seção anterior. Portanto, o vetor de parâmetros $\boldsymbol{\beta}$ é de dimensão $9 \times 1$. Neste caso, $d_{i}$ é o efeito aleatório associado ao $i$-ésimo distrito e a matriz $\mathbf{Z}_{i}$ será considerada como um vetor de uns de dimensão $m_{i}$ e que denotaremos por $\mathbf{1}_{m i}$.

Vamos supor que os erros têm distribuição t-Student e portanto, o vetor $\mathbf{Y}_{i} \sim t_{m_{i}}\left(\mathbf{X}_{i} \boldsymbol{\beta}, \boldsymbol{\Sigma}_{i}, \nu\right)$, em que a matriz $\boldsymbol{\Sigma}_{i}=\alpha \mathbf{I}_{m i}+\gamma \mathbf{J}_{i}$, uma matriz $m_{i} \times m_{i}$, com $\mathbf{I}_{m i}$ sendo uma matriz identidade de ordem $m_{i}$ e $\mathbf{J}_{i}$ uma matriz quadrada $m_{i} \times m_{i}$, cujos elementos são todos uns, para $i=1, \ldots, 15$.

Para determinar os graus de liberdade $\nu$ consideramos a análise sugerida por Borssoi et al. (2017), que consiste na escolha dos graus de liberdade que tenham menor critério de informação de Akaike (1974)(AIC). Na Tabela 6.12 apresentamos os AIC obtidos para distintos graus de liberdade, observamos que o menor valor é obtido quando consideramos 7 graus de liberdade. Portanto, para o ajuste do modelo consideraremos uma distribuição t-Student $\operatorname{com} \nu=7$.

Tabela 6.12: Valores do critério de informação para a distribuição t-Student com diferentes graus de liberdade $\nu$.

\begin{tabular}{|c|c|c|c|c|c|c|}
\hline$\nu$ & 3 & 4 & 5 & 6 & $\mathbf{7}$ & 8 \\
\hline AIC & 87.732 & 87.995 & 88.505 & 88.381 & $\mathbf{8 7 . 5 9 2}$ & 87.831 \\
\hline
\end{tabular}

Na Tabela 6.13 apresentamos as estimativas dos parâmetros obtidas pelos diferentes métodos de estimação, considerando um modelo misto linear e um modelo misto linear t-Student. Lembrando 

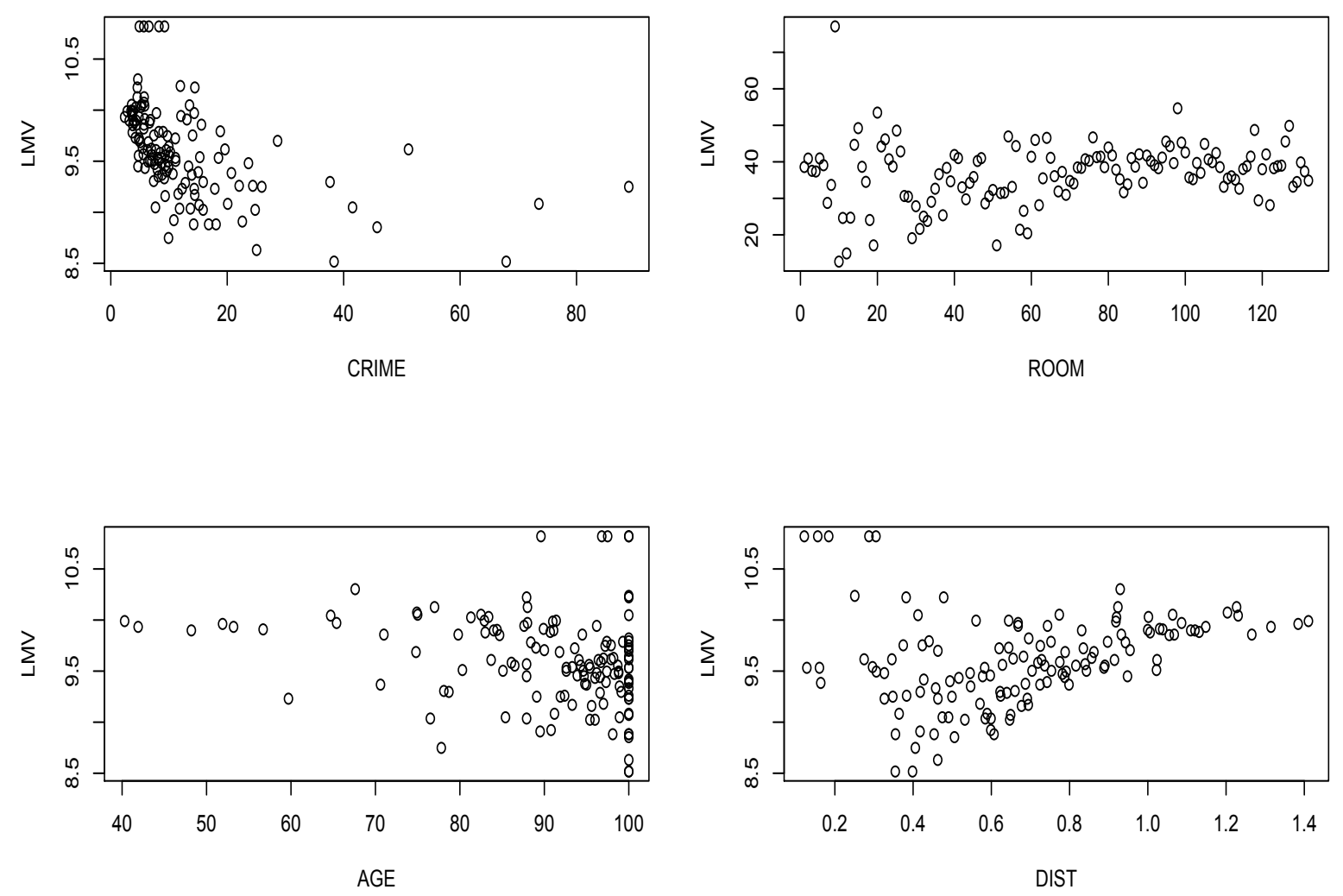

Figura 6.9: Gráficos de dispersão das variáveis CRIME, ROOM, AGE e DIST em relação com à variável $L M V$.

que MV denota as estimativas de máxima verossimilhança, MVR as estimativas de máxima verossimilhança restrita, MVPM estimativas de máxima verossimilhança perfilada modificada e MVPMA estimativas de máxima verossimilhança perfilada modificada aproximada. Vemos que as estimativas obtidas por MV e MVR no modelo normal não apresentam grandes diferenças. As maiores diferenças são dadas nos parâmetros associados às covariáveis DIST e BLACK e ao parâmetro $\gamma$, sendo a variância associada ao efeito aleatório. Comparando esses resultados com as estimativas do modelo t-Student vemos que o parâmetro associado à covariável CHAS é a que apresenta uma maior diferença, mudando de um valor negativo no modelo normal a um aporte positivo no valor da média da variável LMV no modelo t-Student. Na descrição das variáveis feita por Harrison e Rubinfeld (1978) esta covariável tem relação com as comodidades próximas do Rio Charles, portanto sua relação deveria ser positiva. Comparando as estimativas obtidas pelos métodos de estimação do modelo t-Student não vemos grandes variações. Contudo e da mesma forma que a comparação entre as estimativas obtidas no modelo normal, as estimativas que apresentam maior variação são dos parâmetros associados às covariáveis DIST e BLACK e do parâmetro $\gamma$, sendo menores do que as estimativas obtidas pelo método de máxima verossimilhança com respeito aos métodos de MVPM e MVPMA.

Da mesma forma que na aplicação anterior, Seção 6.3.1, vamos avaliar o ajuste dos modelos normal e t-Student, utilizando um gráfico da distância transformadas (ver Little (1988), Lange et al. (1989)). Considerando as distâncias transformadas apresentadas nas equações (6.1) e (6.2), em que $m_{i}$ são os tamanhos dos distritos, para $i=1, \ldots, 15$ e $\nu$ é igual a 7 . Na Figura 6.11 apresentamos os qqplots (gráficos de quantis) das diferenças transformadas do modelo normal e t-Student com respeito à distribuição normal padrão. Vemos que o ajuste b) das distâncias transformadas do modelo t-Student tem uma maior quantidade de pontos sobre a reta, portanto apresenta um ajuste 

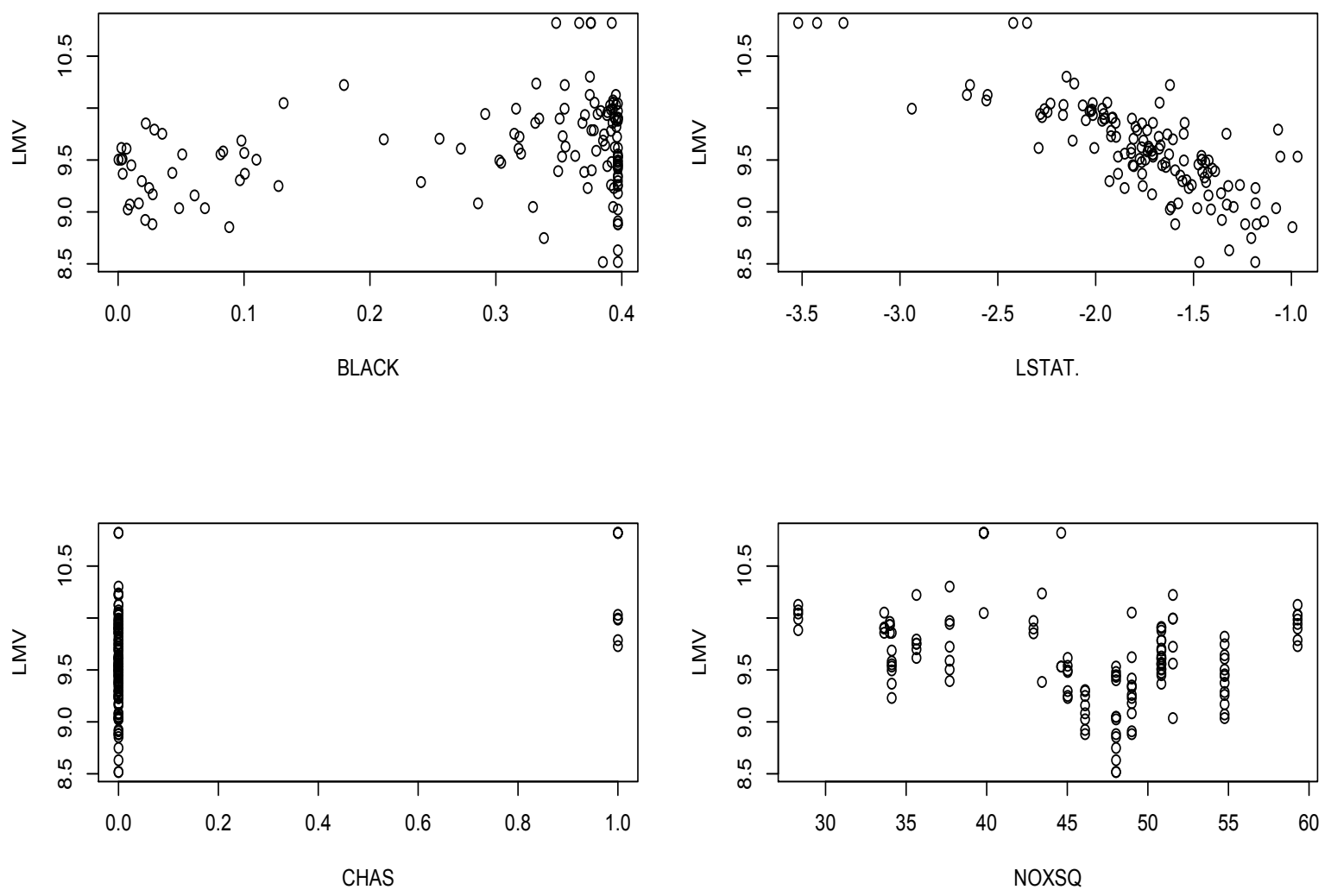

Figura 6.10: Gráficos de dispersão das variáveis BLACK, LSTAT, CHAS e NOXSQ em relação com à variável $L M V$.

Tabela 6.13: Estimativas dos parâmetros considerando o modelo normal e o modelo t-Student, obtidas pelos diferentes métodos de estimação. ${ }^{b}$

\begin{tabular}{|l|rr|rrr|}
\cline { 2 - 6 } \multicolumn{1}{c|}{} & \multicolumn{2}{c|}{ Normal } & \multicolumn{3}{c|}{ t-Student, com $\nu=7$} \\
\cline { 2 - 6 } \multicolumn{1}{c|}{} & \multicolumn{1}{c|}{ MV } & \multicolumn{1}{c|}{ MVR } & \multicolumn{1}{c|}{ MV } & MVPM & MVPMA \\
\hline$\beta_{0}$ & 9.0759 & 9.0662 & 9.0495 & 8.9848 & 8.9726 \\
$\beta_{1}$ (CRIME) & -0.0073 & -0.0072 & -0.0063 & -0.0063 & -0.0063 \\
$\beta_{2}$ (ROOM) & -0.0009 & -0.0007 & -0.0005 & -0.0012 & -0.0012 \\
$\beta_{3}$ (AGE) & 0.0006 & 0.0006 & 0.0010 & 0.0009 & 0.0009 \\
$\beta_{4}$ (DIST) & 0.0172 & 0.0264 & 0.0192 & 0.0900 & 0.0939 \\
$\beta_{5}$ (BLACK) & 0.4477 & 0.4570 & 0.4727 & 0.5007 & 0.5001 \\
$\beta_{6}$ (LSTAT) & -0.5394 & -0.5319 & -0.5418 & -0.5184 & -0.5215 \\
$\beta_{7}$ (CHAS) & -0.0352 & -0.0437 & 0.0032 & 0.0003 & 0.0000 \\
$\beta_{8}$ (NOXSQ) $)$ & -0.0103 & -0.0104 & -0.0109 & -0.0093 & -0.0093 \\
$\alpha$ & 0.0292 & 0.0308 & 0.0239 & 0.0236 & 0.0266 \\
$\gamma$ & 0.0444 & 0.0554 & 0.0250 & 0.0320 & 0.0353 \\
\hline
\end{tabular}

${ }^{b}$ Nas Tabelas F.1 e F.2 apresentamos os desvios padrão considerando o procedimento bootstrap.

mais adequado que o modelo normal.

Por último, realizamos um procedimento bootstrap não paramétrico para analisar o comportamento assintótico das estimativas. Realizamos 500 e 1000 amostras tendo resultados similares. Também consideramos dois possíveis cenários para um distrito ser escolhido no processo de reamostragem. O primeiro é que todos os distritos tenham a mesma probabilidade de ser escolhido. $\mathrm{O}$ segundo, que os distritos de maior tamanho tenham maior probabilidade de ser escolhido. 
a)

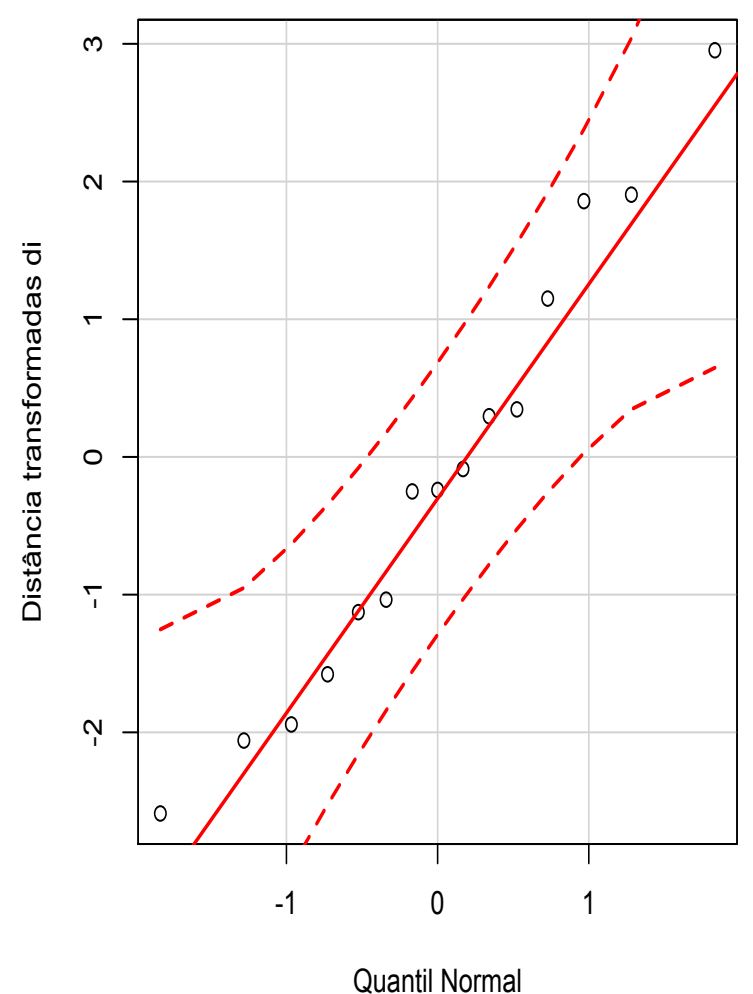

b)

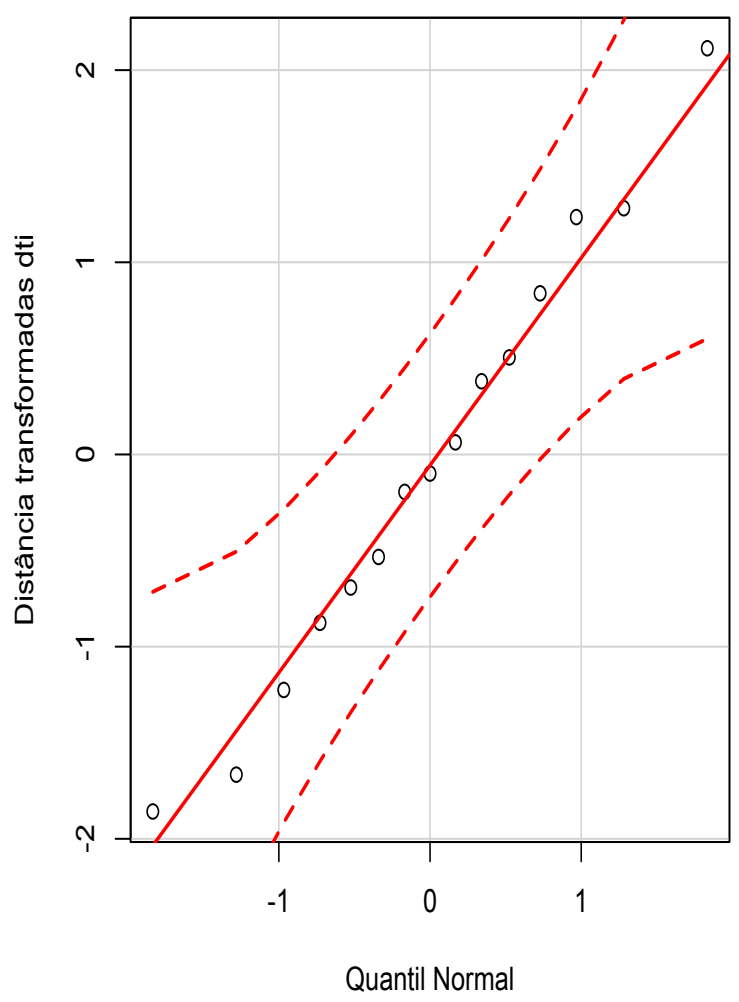

Figura 6.11: qqplots das distâncias transformadas, a) modelo normal e b) modelo t-Student.

Nas Tabelas F.1 e F.2 do Apêndice F.1 apresentamos os resultados de ambos os cenários. Para cada parâmetro foram obtidos a estimativas bootstrap e seu desvio padrão. Em geral, as estimativas têm pouca variabilidade, mas no primeiro cenário a variabilidade das estimativas é levemente maior. O comportamento das estimativas do parâmetro $\alpha$ é similar para ambos os cenários e todos os métodos de estimação. No caso do parâmetro $\gamma$, as estimativas médias obtidas para o primeiro cenário são maiores do que o segundo, assim também os desvios padrão.

\subsection{Comentários}

Na Seção 6.1 apresentamos dois modelos lineares t-Student com duas estruturas de $\boldsymbol{\Sigma}$ diferentes. Vemos que as estimativas de máxima verossimilhança restrita são similares às estimativas de máxima verossimilhança perfilada modificada. Também vemos que as estimativas que apresentam maior discrepância são as de máxima verossimilhança perfilada modificada aproximada. Nos modelos mistos lineares t-Student, apresentados na Seção 6.2, temos, em geral, que as estimativas de máxima verossimilhança e máxima verossimilhança perfilada modificada para o parâmetro $\alpha$ associado ao erro aleatório têm viés relativo similar em todos os modelos e combinação de valores de $\alpha$ e $\gamma$. Enquanto que as estimativas de máxima verossimilhança perfilada modificada aproximadas são mais variáveis mas tendem a ser melhores à medida que $n$ aumenta. Para o parâmetro $\gamma$, associado ao efeito aleatório, vemos que para todos os modelos as estimativas de máxima verossimilhança perfilada modificada têm viés relativo menor do que os outros métodos de estimação. Na Seção 6.3 vimos que as estimativas não apresentam grande variabilidades entre os métodos de estimação de máxima verossimilhança, máxima verossimilhança perfilada modificada e aproximada, mas vimos que o comportamento das estimativas de máxima verossimilhança perfilada modificada e aproximada têm menor variabilidade. 


\section{Capítulo 7}

\section{Discussões}

\subsection{Considerações Finais}

Neste trabalho estudamos a metodologia de máxima verosimilhança restrita amplamente utilizada em modelos mistos. Vista num modelo normal, consiste na transformação da variável em estudo para obter uma função de verossimilhança dos parâmetros da matriz $\boldsymbol{\Sigma}$ que não dependa do vetor $\boldsymbol{\beta}$ mas que considera a perda de informação dos dados ao ter que estimar $\boldsymbol{\beta}$. Visto de forma mais geral, o objetivo é obter uma função de verossimilhança marginal dos parâmetros de interesse que não dependa dos parâmetros de incômodo. O principal objetivo foi estudar a metodologia restrita para propor uma extensão aos modelos mistos lineares elípticos. Como a distribuição normal pertence à família elíptica, inicialmente analisamos a aplicabilidade da metodologia de verosimilhança restrita para o modelo linear elíptico, que tem propriedades similares ao modelo linear normal. Então, estender esta metodologia é imediata, se consideramos a relação dos estimadores de máxima verossimilhança das distribuições elípticas com os estimadores de máxima verossimilhança da distribuição normal apresentados por Anderson et al. (1986). Um aspecto importante nesta metodologia é que as transformações das variáveis com distribuições elípticas continuam sendo elípticas mas não necessariamente com a mesma função geradora de densidade. Outra consideração desta metodologia é que no caso normal conseguimos estimatidores não viesados para os parâmetros associados à matriz $\boldsymbol{\Sigma}$. Porém, isto não pode ser sustentado às outras distribuições da família elíptica.

De forma mais geral, a função de verossimilhança perfilada modificada é uma aproximação à verossimilhança marginal dos parâmetros de interesse. No modelo linear normal a função de verossimilhança perfilada modificada resultante coincide com a função de verossimilhança restrita para os parâmetros da matriz $\boldsymbol{\Sigma}$. Mas para os modelos lineares elípticos isto não pode ser concluído como foi visto no Capítulo 3.

No modelo misto linear elíptico a extensão da metodologia restrita não é direta, devido a que na formulação do modelo, descrito no Capítulo 4, a função de verossimilhança resultante é o produto de densidades elípticas que não necessariamente pertence à família elíptica. Neste aspecto, propusemos uma modificação do método de estimação restrita mas não conseguimos recuperar o caso normal. Assim, usamos a função de verossimilhança perfilada modificada como uma aproximação à verossimilhança marginal dos parâmetros associados à matriz $\boldsymbol{\Sigma}$.

No Capítulo 5, de forma alternativa, propomos estender a aproximação da MVR proposta por Taylor e Verbyla (2006) para uma formulação do modelo misto linear t-Student. Porém, dada sua formulação não é possível ser estendida à família elíptica.

Nos modelos apresentados no Capítulo 6 vimos que as estimativas de máxima verossimilhança perfilada modificada apresentam, em geral, viés relativo igual ou menor às estimativas de máxima verossimilhança. Nas aplicações vimos que as estimativas de máxima verossimilhança perfilada modificada e aproximada têm um comportamento mais estável no procedimento de bootstrap. 


\subsection{Sugestões para pesquisas futuras}

Neste trabalho apresentamos resultados gerais para os modelos lineares elípticos em que o desenvolvimento da metodologia depende das propriedades das distribuições. Vimos que as expressões obtidas considerando a distribuição t-Student são mais complexas do que as obtidas na distribuição normal. No caso da distribuição exponencial potência vimos que sua densidade marginal não continua sendo uma distribuição exponencial potência. Portanto, consideramos relevante analisar esta metodologia para outras distribuições elípticas.

Por outro lado, consideramos que a metodologia de aproximação apresentada no Capítulo 5 pode ser estendida para a classe de distribuições de mistura de escala normal. Consideramos esta aproximação em uma ferramenta útil para diversos modelos.

Analisamos algumas estruturas para a matriz $\Sigma$ e apresentamos suas equações de estimação nos modelos lineares elípticos que podem ser estendidos aos modelos mistos lineares elípticos. Assim, consideramos que isto pode ser utilizado para muitas aplicações.

Nos aspectos de simulações, há muitos cenários em que podemos analisar as estimativas. Por exemplo, no modelo misto linear t-Student, podemos incorporar um maior número de covariáveis ou propor outras estruturas para a matriz dos efeitos aleatórios. 


\section{Apêndice A}

\section{Conceitos preliminares}

\section{A.1 Método de estimação restrita}

Nesta seção apresentamos algumas propriedades matriciais que utilizaremos no desenvolvimento da transformação $\mathbf{W}^{\top} \mathbf{Y}$ proposta por Verbyla (1990) e que foi discutida na Seção 2.3.1. O lema definido a seguir permitirá rescrever algumas matrizes envolvidas na transformação $\mathbf{W}^{\top} \mathbf{Y}$.

Lema A.1.1 Sejam $\mathbf{W}_{2}$ e $\mathbf{X}$ matrizes que satisfazem $\mathbf{W}_{2}^{\top} \mathbf{X}=\mathbf{0}$ e $\boldsymbol{\Sigma}$ uma matriz positiva definida, então

$$
\boldsymbol{\Sigma}-\boldsymbol{\Sigma} \mathbf{W}_{2}\left(\mathbf{W}_{2}^{\top} \boldsymbol{\Sigma} \mathbf{W}_{2}\right)^{-1} \mathbf{W}_{2}^{\top} \boldsymbol{\Sigma}=\mathbf{X}\left(\mathbf{X}^{\top} \boldsymbol{\Sigma}^{-1} \mathbf{X}\right)^{-1} \mathbf{X}^{\top}
$$

Verbyla (1990) considera a prova feita por James (1973).

A dedução do lema é apresentada considerando a consequência que, se temos uma matriz $\mathbf{P}$ com projeção ortogonal $R(\mathbf{P})=\mathbf{P}\left(\mathbf{P}^{\top} \mathbf{P}\right)^{-1} \mathbf{P}^{\top}$, então seu complemento ortogonal será $\mathbf{I}_{m}-R(\mathbf{P})$, em que $\mathbf{I}_{m}$ denota a matriz identidade de ordem $m$.

Além disso, considerando que a matriz $\boldsymbol{\Sigma}$ é simétrica e pela decomposição espectral pode ser escrita como $\mathbf{N} \boldsymbol{\Lambda} \mathbf{N}^{\top}$, em que a matriz $\mathbf{N}$ é uma matriz ortogonal tal que $\mathbf{N}^{\top} \mathbf{N}=\mathbf{N N}^{\top}=\mathbf{I}_{m}$ e $\boldsymbol{\Lambda}$ é uma matriz diagonal que contém os autovalores de $\boldsymbol{\Sigma}$. Portanto, $\boldsymbol{\Sigma}^{s}=\mathbf{N} \boldsymbol{\Lambda}^{s} \mathbf{N}^{\top}$ para todo $s$ em $\mathbb{R}$. Então, temos, por exemplo, que $\boldsymbol{\Sigma}^{-1}=\boldsymbol{\Sigma}^{-1 / 2} \boldsymbol{\Sigma}^{-1 / 2}$.

Agora, se definimos $\mathbf{X}^{*}=\mathbf{\Sigma}^{-1 / 2} \mathbf{X}$ com projeção ortogonal $R\left(\mathbf{X}^{*}\right)$ e seu complemento ortogonal será $\mathbf{I}_{m}-\mathbf{X}^{*}\left(\mathbf{X}^{* T} \mathbf{X}^{*}\right)^{-1} \mathbf{X}^{* T}$, em que $\mathbf{I}_{m}$ denota a matriz identidade de ordem $m$. Desde o lema, $\mathbf{W}_{2}^{\top} \mathbf{X}=\mathbf{0}$, que pode ser interpretado que as colunas de $\mathbf{W}_{2}$ formam uma base do complemento ortogonal de $\mathbf{X}$. Ou seja, o complemento ortogonal de $\mathbf{X}$ pode ser determinado pela projeção de $R\left(\mathbf{W}_{2}\right)$. Assim, podemos definir uma matriz de projeção ortogonal $R\left(\mathbf{W}_{2}^{*}\right)$, com $\mathbf{W}_{2}^{*}=\Sigma^{1 / 2} \mathbf{W}_{2}$. Então, o complemento ortogonal de $\mathbf{X}^{*}$ será,

$$
\begin{aligned}
\mathbf{I}_{m}-\mathbf{X}^{*}\left(\mathbf{X}^{* T} \mathbf{X}^{*}\right)^{-1} \mathbf{X}^{* T} & =R\left(\mathbf{W}_{2}^{*}\right) \\
& =\mathbf{W}_{2}^{*}\left(\mathbf{W}_{2}^{* T} \mathbf{W}_{2}^{*}\right)^{-1} \mathbf{W}_{2}^{* T} .
\end{aligned}
$$

Então, podemos escrever (A.2) com respeito a $\mathbf{X}$ e $\mathbf{W}$ como

$$
\mathbf{I}_{m}-\boldsymbol{\Sigma}^{-1 / 2} \mathbf{X}\left(\mathbf{X}^{\top} \boldsymbol{\Sigma}^{-1} \mathbf{X}\right)^{-1} \mathbf{X}^{\top} \boldsymbol{\Sigma}^{-1 / 2}=\boldsymbol{\Sigma}^{1 / 2} \mathbf{W}_{2}\left(\mathbf{W}_{2}^{\top} \boldsymbol{\Sigma} \mathbf{W}_{2}\right)^{-1} \mathbf{W}_{2}^{\top} \boldsymbol{\Sigma}^{1 / 2} .
$$

Daqui, obtemos três resultados importantes:

- Por um lado, pré e pós multiplicando (A.3) por $\boldsymbol{\Sigma}^{1 / 2}$ obtemos a igualdade do Lema A.1.

- Agora, se pré e pós multiplicamos a equação (A.3) por $\boldsymbol{\Sigma}^{-1 / 2}$, obtemos

$$
\boldsymbol{\Sigma}^{-1}-\boldsymbol{\Sigma}^{-1} \mathbf{X}\left(\mathbf{X}^{\top} \boldsymbol{\Sigma}^{-1} \mathbf{X}\right)^{-1} \mathbf{X}^{\top} \boldsymbol{\Sigma}^{-1}=\mathbf{W}_{2}\left(\mathbf{W}_{2}^{\top} \boldsymbol{\Sigma} \mathbf{W}_{2}\right)^{-1} \mathbf{W}_{2}^{\top} .
$$

Podemos ver que o lado esquerdo desta equação não depende nem de $\mathbf{W}_{1}$ nem de $\mathbf{W}_{2}$. 
- Por último, pré e pós multiplicando a equação (A.3) por $\mathbf{W}_{1}^{\top}$ e $\mathbf{W}_{1}$, respectivamente, obtemos

$$
\mathbf{W}_{1}^{\top} \boldsymbol{\Sigma} \mathbf{W}_{1}-\mathbf{W}_{1}^{\top} \boldsymbol{\Sigma} \mathbf{W}_{2}\left(\mathbf{W}_{2}^{\top} \boldsymbol{\Sigma} \mathbf{W}_{2}\right)^{-1} \mathbf{W}_{2}^{\top} \boldsymbol{\Sigma} \mathbf{W}_{1}=\mathbf{W}_{1}^{\top} \mathbf{X}\left(\mathbf{X}^{\top} \boldsymbol{\Sigma}^{-1} \mathbf{X}\right)^{-1} \mathbf{X}^{\top} \mathbf{W}_{1}
$$

Aqui, a expressão da esquerda tem a estrutura da matriz de variância-covariância de uma distribuição condicional, sendo que a matriz de variância-covariância condicional de $\mathbf{V}_{1}$ dado $\mathbf{V}_{2}$ da transformação definida na Seção 2.3.1 em que $\mathbf{W}^{\top} \mathbf{Y}$ fica dada por

$$
\operatorname{Var}\left(\mathbf{V}_{1}\right)-\operatorname{Cov}\left(\mathbf{V}_{1}, \mathbf{V}_{2}\right) \operatorname{Var}\left(\mathbf{V}_{2}\right)^{-1} \operatorname{Cov}\left(\mathbf{V}_{2}, \mathbf{V}_{1}\right)=\mathbf{W}_{1}^{\top} \mathbf{X}\left(\mathbf{X}^{\top} \boldsymbol{\Sigma}^{-1} \mathbf{X}\right)^{-1} \mathbf{X}^{\top} \mathbf{W}_{1}
$$

Portanto, vemos que a matriz de variância-covariância condicional de $\mathbf{V}_{1}$ dado $\mathbf{V}_{2}$ não depende de $\mathbf{W}_{2}$.

Mais adiante, as expressões (A.4) e (A.5), derivadas do lema, serão utilizadas para facilitar os cálculos na separação da função de verossimilhança.

Para os cálculos dos determinantes, Verbyla (1990) propõe o uso da seguinte propriedade:

Seja a decomposição de

$$
\left|\mathbf{W}^{\top} \boldsymbol{\Sigma} \mathbf{W}\right|=\left|\mathbf{X}^{\top} \boldsymbol{\Sigma}^{-1} \mathbf{X}\right|^{-1}\left|\mathbf{W}_{2}^{\top} \boldsymbol{\Sigma} \mathbf{W}_{2}\right|,
$$

considerando que $\mathbf{W}$ é uma matriz quadrada e $\boldsymbol{\Sigma}$ é uma matriz positiva definida, temos que,

$$
\left|\mathbf{W}^{\top} \mathbf{\Sigma} \mathbf{W}\right|=\left|\mathbf{W}^{\top} \mathbf{W}\right||\boldsymbol{\Sigma}| \text {. }
$$

Então, das equações (A.6) e (A.7) temos que

$$
\left|\mathbf{W}_{2}^{\top} \boldsymbol{\Sigma} \mathbf{W}_{2}\right|=\left|\mathbf{X}^{\top} \boldsymbol{\Sigma}^{-1} \mathbf{X}\right|\left|\mathbf{W}^{\top} \mathbf{W}\right||\boldsymbol{\Sigma}| \text {. }
$$

Desta forma, o determinante da matriz de variância-covariância de $\mathbf{V}_{2}$ pode ser decomposto em três termos, isto também facilitará nossos cálculos da Seção 2.3.2.

\section{A.2 Função de verossimilhança perfilada modificada}

1. Função de verossimilhança perfilada é uma aproximação da função de verossimilhança, que consiste em substituir os parâmetros de incômodo por seus estimadores de máxima verossimilhança (EMV), isto para cada valor fixo do parâmetro de interesse. Seja $L(\boldsymbol{\theta}, \boldsymbol{\eta})$ uma função de verossimilhança com parâmetros $\boldsymbol{\theta}$ e $\boldsymbol{\eta}$ desconhecidos de dimensões $q$ e $p$, respectivamente, com $q+p<m$, em que $\boldsymbol{\theta}$ é o parâmetro de interesse.

Definição A.2.1 Dada a função de verossimilhança conjunta, $L(\boldsymbol{\theta}, \boldsymbol{\eta})$, a verossimilhança perfilada de $\boldsymbol{\theta}$ é definida por

$$
L(\boldsymbol{\theta})=\max _{\boldsymbol{\eta}} L(\boldsymbol{\theta}, \boldsymbol{\eta}),
$$

em que a maximização é calculada para um valor fixo de $\boldsymbol{\theta}$.

Portanto, o estimador de máxima verossimilhança de $\boldsymbol{\eta}$, estará em função de $\boldsymbol{\theta}$. Então, escrevemos

$$
L_{p}(\boldsymbol{\theta})=L\left(\boldsymbol{\theta}, \hat{\boldsymbol{\eta}}_{\theta}\right) .
$$

Não é possível trabalhar diretamente com os estimadores obtidos da função de verossimilhança perfilada, pois a mesma não é uma função de verossimilhança apropriada, no sentido que, não é baseada na probabilidade de alguma quantidade observada. Por outra parte, não tem as propriedades das funções escore, pois a esperança da função escore nem sempre será zero, e a variância dos estimadores não é igual à matriz de informação esperada de Fisher. Isso conduz a estimativas viesadas e a uma sobreestimação dos erros padrão das estimativas. 
2. Aproximação para a distribuição do estimador de máxima verossimilhança, considerando $\hat{\boldsymbol{\eta}}$ o EMV de $\boldsymbol{\eta}$, temos que sua distribuição assintótica fica dada por

$$
\hat{\boldsymbol{\eta}} \stackrel{a}{\sim} N_{p}\left(\boldsymbol{\eta}, \mathbf{J}_{\eta \eta}(\hat{\boldsymbol{\eta}})^{-1}\right),
$$

em que $\mathbf{J}_{\eta \eta}(\hat{\boldsymbol{\eta}})$ representa a matriz de informação observada com respeito ao parâmetro $\boldsymbol{\eta}$, avaliada em $\hat{\boldsymbol{\eta}}$. Assim, a função de densidade aproximada de $\hat{\boldsymbol{\eta}}$ assume a forma

$$
f_{\eta}(\hat{\boldsymbol{\eta}}) \approx\left|\mathbf{J}_{\eta \eta}(\hat{\boldsymbol{\eta}}) /(2 \pi)\right|^{1 / 2} \exp \left\{-(\hat{\boldsymbol{\eta}}-\boldsymbol{\eta})^{\top} \mathbf{J}_{\eta \eta}(\hat{\boldsymbol{\eta}})(\hat{\boldsymbol{\eta}}-\boldsymbol{\eta}) / 2\right\}
$$

Por outro lado, consideremos $D$ como sendo a estatística da razão de verossimihanças, em que

$$
D=2 \log \frac{L(\hat{\boldsymbol{\eta}})}{L(\boldsymbol{\eta})}
$$

sabemos que sua distribuição assintótica (ver, por exemplo, Lee et al. (2006)) é dada por

$$
D \approx(\hat{\boldsymbol{\eta}}-\boldsymbol{\eta})^{\top} \mathbf{J}_{\eta \eta}(\hat{\boldsymbol{\eta}})(\hat{\boldsymbol{\eta}}-\boldsymbol{\eta}) \sim \chi_{p}^{2} .
$$

Neste sentido, podemos usar essa aproximação da forma quadrática, substituindo na equação (A.10) e assim, obter outra aproximação para a densidade de $\hat{\boldsymbol{\eta}}$, dada por

$$
f_{\eta}(\hat{\boldsymbol{\eta}}) \approx\left|\mathbf{J}_{\eta \eta}(\hat{\boldsymbol{\eta}}) /(2 \pi)\right|^{1 / 2} \frac{L(\boldsymbol{\eta})}{L(\hat{\boldsymbol{\eta}})}
$$

Essa última aproximação é denominada verossimilhança baseada em $p$-fórmula que foi proposta por Barndorff-Nielsen (1980). Esse resultado deveria ser entendido como uma maneira de formular a densidade da amostra, em que $\boldsymbol{\eta}$ é fixado e $\hat{\boldsymbol{\eta}}$ varia. Porém, a $p$-fórmula nem sempre tem integral igual a 1. Barndorff-Nielsen (1980) propõe uma fórmula melhorada, denominada $p^{*}$-fórmula que assume a forma

$$
p_{\eta}^{*}(\hat{\boldsymbol{\eta}}) \approx c(\boldsymbol{\eta})\left|\mathbf{J}_{\eta \eta}(\hat{\boldsymbol{\eta}}) /(2 \pi)\right|^{1 / 2} \frac{L(\boldsymbol{\eta})}{L(\hat{\boldsymbol{\eta}})} .
$$

Deveríamos esperar que na maioria dos casos $c(\boldsymbol{\eta})$ seja muito próximo de um, de fato, $c(\hat{\boldsymbol{\eta}}) \approx$ $1+B(\boldsymbol{\eta}) / n$, em que $B(\boldsymbol{\eta})$ é limitado por cima por $n$. Se existe dificuldade na derivação analítica de $c(\boldsymbol{\eta})$, isso pode ser resolvido numericamente. Lee et al. (2006) sugerem que, quando se trabalha com aproximações de funções de verossimilhanças, é mais conveniente trabalhar com a $p$-fórmula.

\section{A.2.1 Método de estimação baseado na função de verossimilhança perfilada}

Para explicar o método, consideraremos um modelo multiparamétrico. Seja $(\hat{\boldsymbol{\theta}}, \hat{\boldsymbol{\eta}})$ os EMVs de $(\boldsymbol{\theta}, \boldsymbol{\eta})$. Desde a equação (A.11) temos a seguinte aproximação para a função de densidade dos estimadores de $(\boldsymbol{\theta}, \boldsymbol{\eta})$ :

$$
f(\hat{\boldsymbol{\theta}}, \hat{\boldsymbol{\eta}}) \approx|\mathbf{J}(\hat{\boldsymbol{\theta}}, \hat{\boldsymbol{\eta}}) /(2 \pi)|^{1 / 2} \frac{L(\boldsymbol{\theta}, \boldsymbol{\eta})}{L(\hat{\boldsymbol{\theta}}, \hat{\boldsymbol{\eta}})}
$$

Seja $\hat{\boldsymbol{\eta}}_{\theta}$ o EMV de $\boldsymbol{\eta}$ para um valor fixo de $\boldsymbol{\theta}$, e $\mathbf{J}_{\eta \eta}\left(\hat{\boldsymbol{\eta}}_{\theta}\right)$ é a correspondente matriz de informação observada e avaliada no estimador de máxima verossimilhança de $\boldsymbol{\eta}$. Então, para um valor fixo de $\boldsymbol{\theta}$ a função de densidade aproximada de $\hat{\boldsymbol{\eta}}_{\theta}$ fica dada por

$$
f\left(\hat{\boldsymbol{\eta}}_{\theta}\right) \approx\left|\mathbf{J}_{\eta \eta}\left(\hat{\boldsymbol{\eta}}_{\theta}\right) /(2 \pi)\right|^{1 / 2} \frac{L(\boldsymbol{\theta}, \boldsymbol{\eta})}{L\left(\boldsymbol{\theta}, \hat{\boldsymbol{\eta}}_{\theta}\right)},
$$


e podemos notar pela equação (A.9), que $L\left(\boldsymbol{\theta}, \hat{\boldsymbol{\eta}}_{\theta}\right)$ é a função de verossimilhança perfilada de $\boldsymbol{\theta}$. Assim, considerando uma mudança de variável, obtemos a função de densidade marginal de $\hat{\boldsymbol{\eta}}$,

$$
\begin{aligned}
f(\hat{\boldsymbol{\eta}}) & =f\left(\hat{\boldsymbol{\eta}}_{\theta}\right)\left|\frac{\partial \hat{\boldsymbol{\eta}}_{\theta}}{\partial \hat{\boldsymbol{\eta}}}\right| \\
& \approx\left|\mathbf{J}_{\eta \eta}\left(\hat{\boldsymbol{\eta}}_{\theta}\right) /(2 \pi)\right|^{1 / 2} \frac{L(\boldsymbol{\theta}, \boldsymbol{\eta})}{L\left(\boldsymbol{\theta}, \hat{\boldsymbol{\eta}}_{\theta}\right)}\left|\frac{\partial \hat{\boldsymbol{\eta}}_{\theta}}{\partial \hat{\boldsymbol{\eta}}}\right| .
\end{aligned}
$$

Então, podemos escrever a função de densidade da distribuição condicional de $\hat{\boldsymbol{\theta}}$ dado $\hat{\boldsymbol{\eta}}$ como

$$
\begin{aligned}
f(\hat{\boldsymbol{\theta}} \mid \hat{\boldsymbol{\eta}}) & =\frac{f(\hat{\boldsymbol{\theta}}, \hat{\boldsymbol{\eta}})}{f(\hat{\boldsymbol{\eta}})} \\
& \approx \frac{|\mathbf{J}(\hat{\boldsymbol{\theta}}, \hat{\boldsymbol{\eta}}) /(2 \pi)|^{1 / 2} \frac{L(\boldsymbol{\theta}, \boldsymbol{\eta})}{L(\hat{\boldsymbol{\theta}}, \hat{\boldsymbol{\eta}})}}{\left|\mathbf{J}_{\eta \eta}\left(\hat{\boldsymbol{\eta}}_{\theta}\right) /(2 \pi)\right|^{1 / 2} \frac{L(\boldsymbol{\theta}, \boldsymbol{\eta})}{L\left(\boldsymbol{\theta}, \hat{\boldsymbol{\eta}}_{\theta}\right)}\left|\frac{\partial \hat{\boldsymbol{\eta}}_{\theta}}{\partial \hat{\boldsymbol{\eta}}}\right|} \\
& \approx|\mathbf{J}(\hat{\boldsymbol{\theta}}, \hat{\boldsymbol{\eta}}) /(2 \pi)|^{1 / 2} \frac{L\left(\boldsymbol{\theta}, \hat{\boldsymbol{\eta}}_{\theta}\right)}{L(\hat{\boldsymbol{\theta}}, \hat{\boldsymbol{\eta}})}\left|\mathbf{J}_{\eta \eta}\left(\hat{\eta}_{\theta}\right) /(2 \pi)\right|^{-1 / 2}\left|\frac{\partial \hat{\boldsymbol{\eta}}}{\partial \hat{\boldsymbol{\eta}}_{\theta}}\right| \\
& \approx f\left(\boldsymbol{\theta}, \hat{\boldsymbol{\eta}}_{\theta}\right)\left|\mathbf{J}_{\eta \eta}\left(\hat{\boldsymbol{\eta}}_{\theta}\right) /(2 \pi)\right|^{-1 / 2}\left|\frac{\partial \hat{\boldsymbol{\eta}}}{\partial \hat{\boldsymbol{\eta}}_{\theta}}\right| .
\end{aligned}
$$

Nessa última expressão, o termo $f\left(\boldsymbol{\theta}, \hat{\boldsymbol{\eta}}_{\theta}\right)$ é a $p$-fórmula proposta por Barndorff-Nielsen (1980). Então, o logaritmo da aproximação da função de verossimilhança condicional de $\boldsymbol{\theta}$ fica dado por,

$$
l_{p m}(\boldsymbol{\theta}) \approx l\left(\boldsymbol{\theta}, \hat{\boldsymbol{\eta}}_{\theta}\right)-\frac{1}{2} \log \left|\mathbf{J}_{\eta \eta}\left(\hat{\boldsymbol{\eta}}_{\theta}\right) /(2 \pi)\right|+\log \left|\frac{\partial \hat{\boldsymbol{\eta}}}{\partial \hat{\boldsymbol{\eta}}_{\theta}}\right| .
$$

Sendo este resultado a função de verossimilhança perfilada modificada proposta por Barndorff-Nielsen (1983). A quantidade $\frac{1}{2} \log \left|\mathbf{J}_{\eta \eta}\left(\hat{\boldsymbol{\eta}}_{\theta}\right) /(2 \pi)\right|$ pode ser interpretada como sendo a informação com respeito a $\boldsymbol{\theta}$ obtida por $\hat{\boldsymbol{\eta}}_{\theta}$ na função de verossimilhança perfilada ordinária. Enquanto que o termo Jacobiano $\left|\frac{\partial \hat{\boldsymbol{\eta}}}{\partial \hat{\boldsymbol{\eta}}_{\theta}}\right|$ se mantém invariante na função de verosimilhança perfilada modificada com respeito às transformações dos parâmetros de incômodo. Em situações de boa sorte, deveríamos ter parâmetros ortogonais no sentido que $\hat{\boldsymbol{\eta}}=\hat{\boldsymbol{\eta}}_{\theta}$, implicando em $\left|\frac{\partial \hat{\boldsymbol{\eta}}}{\partial \hat{\boldsymbol{\eta}}_{\theta}}\right|=1$. Então, esse termo desaparece da equação (A.12). Cox e Reid (1987) provaram que para $\boldsymbol{\theta}$ escalar, é possível definir o parâmetro de incômodo como sendo $\boldsymbol{\eta}$, tal que $\left|\frac{\partial \hat{\boldsymbol{\eta}}}{\partial \hat{\boldsymbol{\eta}}_{\theta}}\right| \approx 1$. Então, um ajuste da verossimilhança perfilada modificada será

$$
l_{p m}(\boldsymbol{\theta}) \equiv l\left(\boldsymbol{\theta}, \hat{\boldsymbol{\eta}}_{\theta}\right)-\frac{1}{2} \log \left|\mathbf{J}_{\eta \eta}\left(\hat{\boldsymbol{\eta}}_{\theta}\right) /(2 \pi)\right| .
$$

Essa fórmula é apresentada no livro de Lee et al. (2006). A notação está relacionada à ênfase de estarmos perfilando a função de verossimilhança $l$, sobre o parâmetro de incômodo $\boldsymbol{\eta}$. Para facilitar os cálculos, Lee et al. (2006) sugerem que pode-se trabalhar com a matriz de informação esperada de Fisher no lugar da matriz de informação observada.

Por último, da Definição 1.3.1 sabemos que o logaritmo da função de verossimilhança perfilada de $\boldsymbol{\theta}, l_{p}\left(\boldsymbol{\theta}, \hat{\boldsymbol{\eta}}_{\theta}\right)$, é a maximização da função $l(\boldsymbol{\theta}, \boldsymbol{\eta})$ com respeito a $\boldsymbol{\eta}$. Podemos ver que,

$$
\max _{\boldsymbol{\eta}} l(\boldsymbol{\theta}, \boldsymbol{\eta})=\max _{\boldsymbol{\eta}} l^{*}(\boldsymbol{\theta}, \boldsymbol{\eta})
$$

em que

$$
l^{*}(\boldsymbol{\theta}, \boldsymbol{\eta}) \equiv l(\boldsymbol{\theta}, \boldsymbol{\eta} ; \mathbf{Y})-\frac{1}{2} \log \left|\mathbf{J}_{\eta \eta}\left(\hat{\boldsymbol{\eta}_{\theta}}\right) /(2 \pi)\right| .
$$


O segundo termo de $l^{*}(\boldsymbol{\theta}, \boldsymbol{\eta})$ não depende de $\boldsymbol{\eta}$, então, maximizar $l^{*}(\boldsymbol{\theta}, \boldsymbol{\eta})$ com respeito a $\boldsymbol{\eta}$ é o mesmo que maximizar o logaritmo da função de verossimilhança $l(\boldsymbol{\theta}, \boldsymbol{\eta}, \mathbf{Y})$ com respeito a $\boldsymbol{\eta}$.

Este ajuste da função de verossimilhança perfilada modificada foi proposto por Lee et al. (2006), que mostram uma aplicação para o caso normal o qual é discutido na Seção 2.4. 
APÊNDICE A 


\section{Apêndice B}

\section{Cálculos de Matrizes}

\section{B.1 Fatorizações de Matrizes}

\section{B.1.1 Termos para a distribuição condicional $V_{1} \mid V_{2}$}

Nesta seção apresentamos os cálculos de alguns termos importantes referentes ao método de transformação proposto por Verbyla (1990).

1. Para a equação (3.11) usaremos a pós multiplicação do Lema 1.4 .1 por $\boldsymbol{\Sigma}^{-1}$. Então, temos que

$$
\begin{aligned}
\mathbf{I}_{m}-\boldsymbol{\Sigma} \mathbf{W}_{2}\left(\mathbf{W}_{2}^{\top} \boldsymbol{\Sigma} \mathbf{W}_{2}\right)^{-1} \mathbf{W}_{2} & =\mathbf{X}\left(\mathbf{X}^{\top} \boldsymbol{\Sigma}^{-1} \mathbf{X}\right)^{-1} \mathbf{X}^{\top} \boldsymbol{\Sigma}^{-1} \\
\boldsymbol{\Sigma} \mathbf{W}_{2}\left(\mathbf{W}_{2}^{\top} \boldsymbol{\Sigma} \mathbf{W}_{2}\right)^{-1} \mathbf{W}_{2} & =\mathbf{I}_{m}-\mathbf{X}\left(\mathbf{X}^{\top} \boldsymbol{\Sigma}^{-1} \mathbf{X}\right)^{-1} \mathbf{X}^{T} \boldsymbol{\Sigma}^{-1}
\end{aligned}
$$

Logo, a equação (3.11) fica dada por

$$
\begin{aligned}
\boldsymbol{\mu}_{1}\left(\mathbf{V}_{2}\right) & =\boldsymbol{\beta}+\mathbf{W}_{1}^{\top} \boldsymbol{\Sigma} \mathbf{W}_{2}\left(\mathbf{W}_{2}^{\top} \boldsymbol{\Sigma} \mathbf{W}_{2}\right)^{-1} \mathbf{W}_{2} \mathbf{Y} \\
& =\boldsymbol{\beta}+\mathbf{W}_{1}^{\top}\left(\mathbf{I}_{m}-\mathbf{X}\left(\mathbf{X}^{\top} \boldsymbol{\Sigma}^{-1} \mathbf{X}\right)^{-1} \mathbf{X}^{\top} \boldsymbol{\Sigma}^{-1}\right) \mathbf{Y}
\end{aligned}
$$

Agora, substituindo a transformação de $\mathbf{W}_{1}^{\top}=\left(\mathbf{X}^{\top} \mathbf{X}\right)^{-1} \mathbf{X}^{\top}$, obtemos que

$$
\begin{aligned}
\boldsymbol{\mu}_{1}\left(\mathbf{V}_{2}\right) & =\boldsymbol{\beta}+\left(\mathbf{X}^{\top} \mathbf{X}\right)^{-1} \mathbf{X}^{\top}\left(\mathbf{I}_{m}-\mathbf{X}\left(\mathbf{X}^{\top} \boldsymbol{\Sigma}^{-1} \mathbf{X}\right)^{-1} \mathbf{X}^{\top} \boldsymbol{\Sigma}^{-1}\right) \mathbf{Y} \\
& =\boldsymbol{\beta}+\left(\mathbf{X}^{\top} \mathbf{X}\right)^{-1} \mathbf{X}^{\top} \mathbf{Y}-\left(\mathbf{X}^{\top} \mathbf{X}\right)^{-1} \mathbf{X}^{\top} \mathbf{X}\left(\mathbf{X}^{\top} \boldsymbol{\Sigma}^{-1} \mathbf{X}\right)^{-1} \mathbf{X}^{\top} \boldsymbol{\Sigma}^{-1} \mathbf{Y} \\
& =\boldsymbol{\beta}+\left(\mathbf{X}^{\top} \mathbf{X}\right)^{-1} \mathbf{X}^{\top} \mathbf{Y}-\left(\mathbf{X}^{\top} \boldsymbol{\Sigma}^{-1} \mathbf{X}\right)^{-1} \mathbf{X}^{\top} \boldsymbol{\Sigma}^{-1} \mathbf{Y}
\end{aligned}
$$

Assim, a expressão para a média da distribuição condicional de $\mathbf{V}_{1} \mid \mathbf{V}_{2}$ pode ser expressa em termos de componentes do modelo sem depender da transformação $\mathbf{W}_{2}$. Também podemos ver que $\mathbf{W}_{1}^{\top} \mathbf{X}=\left(\mathbf{X}^{\top} \mathbf{X}\right)^{-1} \mathbf{X}^{\top} \mathbf{X}=\mathbf{I}_{p}$, e simplificar a matriz de variância-covariância da distribuição condicional

$$
\begin{aligned}
\mathbf{W}_{1}^{\top} \mathbf{X}\left(\mathbf{X}^{\top} \boldsymbol{\Sigma}^{-1} \mathbf{X}\right)^{-1} \mathbf{X}^{\top} \mathbf{W}_{1} & =\left(\mathbf{X}^{\top} \mathbf{X}\right)^{-1} \mathbf{X}^{\top} \mathbf{X}\left(\mathbf{X}^{\top} \boldsymbol{\Sigma}^{-1} \mathbf{X}\right)^{-1} \mathbf{X}^{\top} \mathbf{X}\left(\mathbf{X}^{\top} \mathbf{X}\right)^{-1} \\
& =\left(\mathbf{X}^{\top} \boldsymbol{\Sigma}^{-1} \mathbf{X}\right)^{-1}
\end{aligned}
$$

Vemos que a matriz de variância-covariância fica bastante reduzida. 
2. Usando o resultado da equação (B.1) e sabendo que $\mathbf{V}_{1}=\mathbf{W}_{1}^{\top} \mathbf{Y}$, podemos escrever

$$
\begin{aligned}
\left(\mathbf{V}_{1}-\boldsymbol{\mu}_{1}\left(\mathbf{V}_{2}\right)\right) & =\mathbf{W}_{1}^{\top} \mathbf{Y}-\boldsymbol{\beta}-\left(\mathbf{X}^{\top} \mathbf{X}\right)^{-1} \mathbf{X}^{\top} \mathbf{Y}+\left(\mathbf{X}^{\top} \boldsymbol{\Sigma}^{-1} \mathbf{X}\right)^{-1} \mathbf{X}^{T} \boldsymbol{\Sigma}^{-1} \mathbf{Y} \\
& =\left(\mathbf{X}^{\top} \mathbf{X}\right)^{-1} \mathbf{X}^{\top} \mathbf{Y}-\left(\mathbf{X}^{\top} \mathbf{X}\right)^{-1} \mathbf{X}^{\top} \mathbf{Y}+\left(\mathbf{X}^{\top} \boldsymbol{\Sigma}^{-1} \mathbf{X}\right)^{-1} \mathbf{X}^{T} \boldsymbol{\Sigma}^{-1} \mathbf{Y}-\boldsymbol{\beta} \\
& =\left(\mathbf{X}^{\top} \boldsymbol{\Sigma}^{-1} \mathbf{X}\right)^{-1} \mathbf{X}^{T} \boldsymbol{\Sigma}^{-1} \mathbf{Y}-\boldsymbol{\beta} \\
& =\mathbf{b}-\boldsymbol{\beta} .
\end{aligned}
$$

Note que o termo chamado b é o estimador de máxima verossimilhança de $\boldsymbol{\beta}$.

3. Portanto, a forma quadrática da verossimilhança da distribuição condicional (2.7) fica expressa na seguinte forma:

$$
\left(\mathbf{V}_{1}-\boldsymbol{\mu}_{1}\left(\mathbf{V}_{2}\right)\right)^{\top}\left(\left(\mathbf{X}^{\top} \boldsymbol{\Sigma}^{-1} \mathbf{X}\right)^{-1}\right)^{-1}\left(\mathbf{V}_{1}-\boldsymbol{\mu}_{1}\left(\mathbf{V}_{2}\right)\right)=(\mathbf{b}-\boldsymbol{\beta})^{\top}\left(\mathbf{X}^{\top} \boldsymbol{\Sigma}^{-1} \mathbf{X}\right)(\mathbf{b}-\boldsymbol{\beta}) .
$$

\section{B.1.2 Outras fatorizações}

Um resultado importante utilizado na função de verossimilhança perfilada modificada é quando a expressão da matriz de informação de $\boldsymbol{\beta}$ avaliada no estimador de máxima verossimilhança $\hat{\boldsymbol{\beta}}_{\alpha}$ fica simplificada ao desenvolver o seguinte termo:

$$
\mathbf{X}^{\top} \boldsymbol{\Sigma}^{-1}\left(\mathbf{Y}-\mathbf{X} \hat{\boldsymbol{\beta}}_{\alpha}\right)=\left(\mathbf{X}^{\top} \boldsymbol{\Sigma}^{-1} \mathbf{Y}-\mathbf{X}^{\top} \boldsymbol{\Sigma}^{-1} \mathbf{X} \hat{\boldsymbol{\beta}}_{\alpha}\right) .
$$

Lembrando que $\hat{\boldsymbol{\beta}}_{\alpha}$ fica dado por $\left(\mathbf{X}^{\top} \boldsymbol{\Sigma}^{-1} \mathbf{X}\right)^{-1} \mathbf{X}^{\top} \boldsymbol{\Sigma}^{-1} \mathbf{Y}$, então temos que

$$
\begin{aligned}
\left(\mathbf{X}^{\top} \boldsymbol{\Sigma}^{-1} \mathbf{Y}-\mathbf{X}^{\top} \boldsymbol{\Sigma}^{-1} \mathbf{X} \hat{\boldsymbol{\beta}}_{\alpha}\right) & =\left(\mathbf{X}^{\top} \boldsymbol{\Sigma}^{-1} \mathbf{Y}-\mathbf{X}^{\top} \boldsymbol{\Sigma}^{-1} \mathbf{X}\left(\mathbf{X}^{\top} \boldsymbol{\Sigma}^{-1} \mathbf{X}\right)^{-1} \mathbf{X}^{\top} \boldsymbol{\Sigma}^{-1} \mathbf{Y}\right) \\
& =\left(\mathbf{X}^{\top} \boldsymbol{\Sigma}^{-1} \mathbf{Y}-\mathbf{I}_{p} \mathbf{X}^{\top} \boldsymbol{\Sigma}^{-1} \mathbf{Y}\right) \\
& =\left(\mathbf{X}^{\top} \boldsymbol{\Sigma}^{-1} \mathbf{Y}-\mathbf{X}^{\top} \boldsymbol{\Sigma}^{-1} \mathbf{Y}\right) \\
& =\mathbf{0}
\end{aligned}
$$

\section{B.2 Cálculos das derivadas de um elemento do vetor $\alpha$}

A seguir apresentamos os cálculos de algumas derivadas que serão usados no trabalho. Consideremos que o vetor $\boldsymbol{\alpha}$ contém os parâmetros que compõem a matriz de variância-covariância $\boldsymbol{\Sigma}$, então $\alpha_{j}$ corresponde ao $j$-ésimo elemento do vetor $\boldsymbol{\alpha}$ para $j=1, \ldots, q$.

1. Igualdade da função escore do logaritmo da função de verossimilhança perfilada modificada equação (2.11) com respeito de $\alpha_{j}$, temos que

$$
\begin{array}{r}
\frac{\partial}{\partial \alpha_{j}}\left(\mathbf{Y}-\mathbf{X} \hat{\boldsymbol{\beta}}_{\alpha}\right)^{\top} \boldsymbol{\Sigma}^{-1}\left(\mathbf{Y}-\mathbf{X} \hat{\boldsymbol{\beta}}_{\alpha}\right)=\frac{\partial}{\partial \alpha_{j}}\left(\mathbf{Y}^{\top} \boldsymbol{\Sigma}^{-1} \mathbf{Y}-\mathbf{Y}^{\top} \boldsymbol{\Sigma}^{-1} \mathbf{X} \hat{\boldsymbol{\beta}}_{\alpha}\right) \\
=\frac{\partial}{\partial \alpha_{j}}\left(\mathbf{Y}^{\top} \boldsymbol{\Sigma}^{-1} \mathbf{Y}-\mathbf{Y}^{\top} \boldsymbol{\Sigma}^{-1} \mathbf{X}\left(\mathbf{X}^{\top} \boldsymbol{\Sigma}^{-1} \mathbf{X}\right)^{-1} \mathbf{X}^{\top} \boldsymbol{\Sigma}^{-1} \mathbf{Y}\right) \\
=-\mathbf{Y}^{\top} \boldsymbol{\Sigma}^{-1} \frac{\partial \boldsymbol{\Sigma}}{\partial \alpha_{j}} \boldsymbol{\Sigma}^{-1} \mathbf{Y}+2 \mathbf{Y}^{\top} \boldsymbol{\Sigma}^{-1} \frac{\partial \boldsymbol{\Sigma}}{\partial \alpha_{j}} \boldsymbol{\Sigma}^{-1} \mathbf{X}\left(\mathbf{X}^{\top} \boldsymbol{\Sigma}^{-1} \mathbf{X}\right)^{-1} \mathbf{X}^{\top} \boldsymbol{\Sigma}^{-1} \mathbf{Y} \\
-\mathbf{Y}^{\top} \boldsymbol{\Sigma}^{-1} \mathbf{X}\left(\mathbf{X}^{\top} \boldsymbol{\Sigma}^{-1} \mathbf{X}\right)^{-1} \mathbf{X}^{\top} \boldsymbol{\Sigma}^{-1} \frac{\partial \boldsymbol{\Sigma}}{\partial \alpha_{j}} \boldsymbol{\Sigma}^{-1} \mathbf{X}\left(\mathbf{X}^{\top} \boldsymbol{\Sigma}^{-1} \mathbf{X}\right)^{-1} \mathbf{X}^{\top} \boldsymbol{\Sigma}^{-1} \mathbf{Y} \\
=-\mathbf{Y}^{\top} \boldsymbol{\Sigma}^{-1} \frac{\partial \boldsymbol{\Sigma}}{\partial \alpha_{j}} \boldsymbol{\Sigma}^{-1} \mathbf{Y}+2 \mathbf{Y}^{\top} \boldsymbol{\Sigma}^{-1} \frac{\partial \boldsymbol{\Sigma}}{\partial \alpha_{j}} \boldsymbol{\Sigma}^{-1} \mathbf{X} \hat{\boldsymbol{\beta}}_{\alpha}-\hat{\boldsymbol{\beta}}_{\alpha}^{\top} \mathbf{X}^{\top} \boldsymbol{\Sigma}^{-1} \frac{\partial \boldsymbol{\Sigma}}{\partial \alpha_{j}} \boldsymbol{\Sigma}^{-1} \mathbf{X} \hat{\boldsymbol{\beta}}_{\alpha} \\
=\left(\mathbf{Y}-\mathbf{X} \hat{\boldsymbol{\beta}}_{\alpha}\right)^{\top} \boldsymbol{\Sigma}^{-1} \frac{\partial \boldsymbol{\Sigma}}{\partial \alpha_{j}} \boldsymbol{\Sigma}^{-1}\left(\mathbf{Y}-\mathbf{X} \hat{\boldsymbol{\beta}}_{\alpha}\right),
\end{array}
$$


para $j=1, \ldots, q$.

2. A derivada do termo (B.2) fica dada por

$$
\frac{\partial}{\partial \alpha_{j}}\left(\mathbf{X}^{\top} \boldsymbol{\Sigma}^{-1} \mathbf{X}\right)^{-1}=\left(\mathbf{X}^{\top} \boldsymbol{\Sigma}^{-1} \mathbf{X}\right)^{-1} \mathbf{X}^{\top} \boldsymbol{\Sigma}^{-1} \frac{\partial \boldsymbol{\Sigma}}{\partial \alpha_{j}} \boldsymbol{\Sigma}^{-1} \mathbf{X}\left(\mathbf{X}^{\top} \boldsymbol{\Sigma}^{-1} \mathbf{X}\right)^{-1}
$$

3. Derivando a equação (B.3) com respeito de $\alpha_{j}$, temos que

$$
\begin{aligned}
\frac{\partial}{\partial \alpha_{j}}\left(\mathbf{V}_{1}-\boldsymbol{\mu}_{1}\left(\mathbf{V}_{2}\right)\right) & =\frac{\partial}{\partial \alpha_{j}}(\mathbf{b}-\boldsymbol{\beta})=\frac{\partial}{\partial \alpha_{j}} \mathbf{b} \\
& =\frac{\partial}{\partial \alpha_{j}}\left(\mathbf{X}^{\top} \boldsymbol{\Sigma}^{-1} \mathbf{X}\right)^{-1} \mathbf{X}^{T} \boldsymbol{\Sigma}^{-1} \mathbf{Y} \\
& =\left(\mathbf{X}^{\top} \boldsymbol{\Sigma}^{-1} \mathbf{X}\right)^{-1}\left(\mathbf{X}^{\top} \boldsymbol{\Sigma}^{-1} \frac{\partial \boldsymbol{\Sigma}}{\partial \alpha_{j}} \boldsymbol{\Sigma}^{-1} \mathbf{X} \mathbf{b}-\mathbf{X}^{\top} \boldsymbol{\Sigma}^{-1} \frac{\partial \boldsymbol{\Sigma}}{\partial \alpha_{j}} \boldsymbol{\Sigma}^{-1} \mathbf{Y}\right)
\end{aligned}
$$

4. Agora para derivar a forma quadrática da equação (B.4) com respeito a $\alpha_{j}$, temos que

$$
\begin{aligned}
\frac{\partial}{\partial \alpha_{j}}(\mathbf{b}-\boldsymbol{\beta})^{\top}\left(\mathbf{X}^{\top} \boldsymbol{\Sigma}^{-1} \mathbf{X}\right)(\mathbf{b}-\boldsymbol{\beta})= & \left(\mathbf{X}^{\top} \boldsymbol{\Sigma}^{-1} \frac{\partial \boldsymbol{\Sigma}}{\partial \alpha_{j}} \boldsymbol{\Sigma}^{-1} \mathbf{X} \mathbf{b}-\mathbf{X}^{\top} \boldsymbol{\Sigma}^{-1} \frac{\partial \boldsymbol{\Sigma}}{\partial \alpha_{j}} \boldsymbol{\Sigma}^{-1} \mathbf{Y}\right) \\
& \times\left(\mathbf{X}^{\top} \boldsymbol{\Sigma}^{-1} \mathbf{X}\right)^{-1}\left(\mathbf{X}^{\top} \boldsymbol{\Sigma}^{-1} \mathbf{X}\right)(\mathbf{b}-\boldsymbol{\beta}) \\
& -(\mathbf{b}-\boldsymbol{\beta})^{\top}\left(\mathbf{X}^{\top} \boldsymbol{\Sigma}^{-1} \frac{\partial \boldsymbol{\Sigma}}{\partial \alpha_{j}} \boldsymbol{\Sigma}^{-1} \mathbf{X}\right)(\mathbf{b}-\boldsymbol{\beta}) \\
& +(\mathbf{b}-\boldsymbol{\beta})^{\top}\left(\mathbf{X}^{\top} \boldsymbol{\Sigma}^{-1} \mathbf{X}\right)\left(\mathbf{X}^{\top} \boldsymbol{\Sigma}^{-1} \mathbf{X}\right)^{-1} \\
& \times\left(\mathbf{X}^{\top} \boldsymbol{\Sigma}^{-1} \frac{\partial \boldsymbol{\Sigma}}{\partial \alpha_{j}} \boldsymbol{\Sigma}^{-1} \mathbf{X} \mathbf{b}-\mathbf{X}^{\top} \boldsymbol{\Sigma}^{-1} \frac{\partial \boldsymbol{\Sigma}}{\partial \alpha_{j}} \boldsymbol{\Sigma}^{-1} \mathbf{Y}\right) \\
= & 2\left(\mathbf{X}^{\top} \boldsymbol{\Sigma}^{-1} \frac{\partial \boldsymbol{\Sigma}}{\partial \alpha_{j}} \boldsymbol{\Sigma}^{-1} \mathbf{X} \mathbf{b}-\mathbf{X}^{\top} \boldsymbol{\Sigma}^{-1} \frac{\partial \boldsymbol{\Sigma}}{\partial \alpha_{j}} \boldsymbol{\Sigma}^{-1} \mathbf{Y}\right)(\mathbf{b}-\boldsymbol{\beta}) \\
- & (\mathbf{b}-\boldsymbol{\beta})^{\top}\left(\mathbf{X}^{\top} \boldsymbol{\Sigma}^{-1} \frac{\partial \boldsymbol{\Sigma}}{\partial \alpha_{j}} \boldsymbol{\Sigma}^{-1} \mathbf{X}\right)(\mathbf{b}-\boldsymbol{\beta}) .
\end{aligned}
$$

5. Derivando a matriz de variância-covariância da distribuição marginal de $\mathbf{V}_{2}$ com respeito a $\alpha_{j}$ considerando usando o a expressão de $\mathbf{b}$, obtemos

$$
\begin{aligned}
\frac{\partial}{\partial \alpha_{j}} \mathbf{V}_{2}^{\top}\left(\mathbf{W}_{2}^{\top} \boldsymbol{\Sigma} \mathbf{W}_{2}\right) \mathbf{V}_{2}= & \frac{\partial}{\partial \alpha_{j}} \mathbf{Y}^{\top} \mathbf{W}_{2}\left(\mathbf{W}_{2}^{\top} \boldsymbol{\Sigma} \mathbf{W}_{2}\right) \mathbf{W}_{2}^{\top} \mathbf{Y} \\
= & \frac{\partial}{\partial \alpha_{j}} \mathbf{Y}^{\top}\left(\boldsymbol{\Sigma}^{-1}-\boldsymbol{\Sigma}^{-1} \mathbf{X}\left(\mathbf{X}^{\top} \boldsymbol{\Sigma}^{-1} \mathbf{X}\right)^{-1} \mathbf{X}^{\top} \boldsymbol{\Sigma}^{-1}\right) \mathbf{Y} \\
= & \mathbf{Y}^{\top} \boldsymbol{\Sigma}^{-1} \frac{\partial \boldsymbol{\Sigma}}{\partial \alpha_{j}} \boldsymbol{\Sigma}^{-1} \mathbf{Y}+\mathbf{Y}^{\top} \boldsymbol{\Sigma}^{-1} \frac{\partial \boldsymbol{\Sigma}}{\partial \alpha_{j}} \boldsymbol{\Sigma}^{-1} \mathbf{X} \mathbf{b} \\
& -\mathbf{b}\left(\mathbf{X}^{\top} \boldsymbol{\Sigma}^{-1} \frac{\partial \boldsymbol{\Sigma}}{\partial \alpha_{j}} \boldsymbol{\Sigma}^{-1} \mathbf{X} \mathbf{b}-\mathbf{X}^{\top} \boldsymbol{\Sigma}^{-1} \frac{\partial \boldsymbol{\Sigma}}{\partial \alpha_{j}} \boldsymbol{\Sigma}^{-1} \mathbf{Y}\right) \\
= & -(\mathbf{Y}-\mathbf{X} \mathbf{b})^{\top} \boldsymbol{\Sigma}^{-1} \frac{\partial \boldsymbol{\Sigma}}{\partial \alpha_{j}} \boldsymbol{\Sigma}^{-1}(\mathbf{Y}-\mathbf{X b}) .
\end{aligned}
$$

\section{B.3 Cálculos das derivadas da matriz escala}

Nesta seção detalhamos algumas derivadas e funções das estruturas de matriz escala que consideramos no Capítulo 3. 
1. Para a estrutura da matriz $\boldsymbol{\Sigma}=\sigma^{2} \boldsymbol{\Sigma}_{c}$, em que $\boldsymbol{\Sigma}_{c}$ é uma matriz simétrica, positiva definida e conhecida, ou seja não depende de $\sigma^{2}$. A inversa de $\boldsymbol{\Sigma}$ fica dada por $\boldsymbol{\Sigma}^{-1}=\left(1 / \sigma^{2}\right) \boldsymbol{\Sigma}_{c}^{-1}$ e temos que a derivada parcial com respeito a $\sigma^{2}$ fica dada por

$$
\frac{\partial \boldsymbol{\Sigma}}{\partial \sigma^{2}}=\frac{\partial}{\partial \sigma^{2}}\left[\sigma^{2} \boldsymbol{\Sigma}_{c}\right]=\boldsymbol{\Sigma}_{c}
$$

Algumas funções das derivadas parciais que utilizaremos nos casos particulares do Capítulo 3 , dadas por

$$
\begin{aligned}
& \boldsymbol{\Sigma}^{-1} \frac{\partial \boldsymbol{\Sigma}}{\partial \sigma^{2}}=\frac{1}{\sigma^{2}} \boldsymbol{\Sigma}_{c}^{-1} \frac{\partial}{\partial \sigma^{2}}\left[\sigma^{2} \boldsymbol{\Sigma}_{c}\right]=\frac{1}{\sigma^{2}} \mathbf{I}_{m}, \\
& \boldsymbol{\Sigma}^{-1} \frac{\partial \boldsymbol{\Sigma}}{\partial \sigma^{2}} \boldsymbol{\Sigma}^{-1}=\frac{1}{\sigma^{2}} \boldsymbol{\Sigma}_{c}^{-1} \frac{\partial}{\partial \sigma^{2}}\left[\sigma^{2} \boldsymbol{\Sigma}_{c}\right] \frac{1}{\sigma^{2}} \boldsymbol{\Sigma}_{c}^{-1}=\frac{1}{\left(\sigma^{2}\right)^{2}} \boldsymbol{\Sigma}_{c}^{-1}, \\
& \operatorname{tr}\left(\boldsymbol{\Sigma}^{-1} \frac{\partial \boldsymbol{\Sigma}}{\partial \sigma^{2}}\right)=\operatorname{tr}\left(\frac{1}{\sigma^{2}} \mathbf{I}_{m}\right)=\frac{1}{\sigma^{2}} m, \\
& \operatorname{tr}\left\{\left(\mathbf{X}^{\top} \boldsymbol{\Sigma}^{-1} \mathbf{X}\right)^{-1} \mathbf{X}^{\top} \boldsymbol{\Sigma}^{-1} \frac{\partial \boldsymbol{\Sigma}}{\partial \sigma^{2}} \boldsymbol{\Sigma}^{-1} \mathbf{X}\right\}=\frac{\sigma^{2}}{\left(\sigma^{2}\right)^{2}} \operatorname{tr}\left\{\left(\mathbf{X}^{\top} \boldsymbol{\Sigma}_{c}^{-1} \mathbf{X}\right)^{-1} \mathbf{X}^{\top} \boldsymbol{\Sigma}_{c}^{-1} \mathbf{X}\right\} \\
& =\frac{1}{\sigma^{2}} \operatorname{tr}\left(\mathbf{I}_{p}\right)=\frac{p}{\sigma^{2}}
\end{aligned}
$$

$\mathrm{e}$

$$
(\mathbf{Y}-\mathbf{X} \boldsymbol{\beta})^{\top} \boldsymbol{\Sigma}^{-1} \frac{\partial \boldsymbol{\Sigma}}{\partial \sigma^{2}} \boldsymbol{\Sigma}^{-1}(\mathbf{Y}-\mathbf{X} \boldsymbol{\beta})=\frac{1}{\left(\sigma^{2}\right)^{2}}(\mathbf{Y}-\mathbf{X} \boldsymbol{\beta})^{\top} \boldsymbol{\Sigma}_{c}^{-1}(\mathbf{Y}-\mathbf{X} \boldsymbol{\beta}) .
$$

Um caso particular é quando a matriz $\boldsymbol{\Sigma}_{c}$ é identidade $\mathbf{I}_{m}$, então a estrutura da matriz $\boldsymbol{\Sigma}=\sigma^{2} \mathbf{I}_{m}$, em que sua inversa fica dada por $\boldsymbol{\Sigma}^{-1}=\left(1 / \sigma^{2}\right) \mathbf{I}_{m}$. Assim, temos que a derivada parcial com respeito a $\sigma^{2}$ fica dada por

$$
\frac{\partial \boldsymbol{\Sigma}}{\partial \sigma^{2}}=\frac{\partial}{\partial \sigma^{2}}\left[\sigma^{2} \mathbf{I}_{m}\right]=\mathbf{I}_{m}
$$

e as funções das derivadas parciais são dadas por

$$
\begin{aligned}
& \boldsymbol{\Sigma}^{-1} \frac{\partial \boldsymbol{\Sigma}}{\partial \sigma^{2}}=\frac{1}{\sigma^{2}} \mathbf{I}_{m} \frac{\partial}{\partial \sigma^{2}}\left[\sigma^{2} \mathbf{I}_{m}\right]=\frac{1}{\sigma^{2}} \mathbf{I}_{m}, \\
& \boldsymbol{\Sigma}^{-1} \frac{\partial \boldsymbol{\Sigma}}{\partial \sigma^{2}} \boldsymbol{\Sigma}^{-1}=\frac{1}{\sigma^{2}} \mathbf{I}_{m} \frac{\partial}{\partial \sigma^{2}}\left[\sigma^{2} \mathbf{I}_{m}\right] \frac{1}{\sigma^{2}} \mathbf{I}_{m}=\frac{1}{\left(\sigma^{2}\right)^{2}} \mathbf{I}_{m}, \\
& \operatorname{tr}\left(\boldsymbol{\Sigma}^{-1} \frac{\partial \boldsymbol{\Sigma}}{\partial \sigma^{2}}\right)=\operatorname{tr}\left(\left(1 / \sigma^{2}\right) \mathbf{I}_{m} \frac{\partial}{\partial \sigma^{2}}\left[\sigma^{2} \mathbf{I}_{m}\right]\right)=\frac{1}{\sigma^{2}} m, \\
& \operatorname{tr}\left\{\left(\mathbf{X}^{\top} \boldsymbol{\Sigma}^{-1} \mathbf{X}\right)^{-1} \mathbf{X}^{\top} \boldsymbol{\Sigma}^{-1} \frac{\partial \boldsymbol{\Sigma}}{\partial \sigma^{2}} \boldsymbol{\Sigma}^{-1} \mathbf{X}\right\}=\frac{\sigma^{2}}{\left(\sigma^{2}\right)^{2}} \operatorname{tr}\left\{\left(\mathbf{X}^{\top} \mathbf{I}_{m} \mathbf{X}\right)^{-1} \mathbf{X}^{\top} \mathbf{I}_{m} \mathbf{X}\right\} \\
& =\frac{1}{\sigma^{2}} \operatorname{tr}\left(\mathbf{I}_{p}\right)=\frac{p}{\sigma^{2}}
\end{aligned}
$$


e

$$
\begin{aligned}
(\mathbf{Y}-\mathbf{X} \boldsymbol{\beta})^{\top} \boldsymbol{\Sigma}^{-1} \frac{\partial \boldsymbol{\Sigma}}{\partial \sigma^{2}} \boldsymbol{\Sigma}^{-1}(\mathbf{Y}-\mathbf{X} \boldsymbol{\beta}) & =(\mathbf{Y}-\mathbf{X} \boldsymbol{\beta})^{\top} \frac{1}{\sigma^{2}} \mathbf{I}_{m} \frac{\partial}{\partial \sigma^{2}}\left[\sigma^{2} \mathbf{I}_{m}\right] \frac{1}{\sigma^{2}} \mathbf{I}_{m}(\mathbf{Y}-\mathbf{X} \boldsymbol{\beta}) \\
& =\frac{1}{\left(\sigma^{2}\right)^{2}}(\mathbf{Y}-\mathbf{X} \boldsymbol{\beta})^{\top}(\mathbf{Y}-\mathbf{X} \boldsymbol{\beta})
\end{aligned}
$$

2. Considerando a matriz $\boldsymbol{\Sigma}$ como uma combinação linear de matrizes da forma $\sum_{j=1}^{q} \alpha_{j} \mathbf{G}_{j}$, a matriz $\mathbf{G}_{j}$ é conhecida de dimensão $m \times m$, cuja estrutura é dada por uma matriz diagonal por bloco que contém uma matriz $\boldsymbol{\Sigma}_{j}$ naj-ésima posição com $j=1, \ldots, q$. A matriz $\boldsymbol{\Sigma}_{j}$ é simétrica e positiva definida de dimensão $\left(m_{j} \times m_{j}\right)$ para $j=1, \ldots, q$ em que $q<m-p$. Portanto, podemos escrever a matriz $\boldsymbol{\Sigma}$ na forma

$$
\boldsymbol{\Sigma}=\alpha_{1} \mathbf{G}_{1}+\ldots+\alpha_{j} \mathbf{G}_{j}+\ldots+\alpha_{q} \mathbf{G}_{q}=\left[\begin{array}{cccccc}
\alpha_{1} \boldsymbol{\Sigma}_{1} & \mathbf{0} & \ldots & \ldots & \ldots & \mathbf{0} \\
\mathbf{0} & \alpha_{2} \boldsymbol{\Sigma}_{2} & \mathbf{0} & \ldots & \ldots & \vdots \\
\vdots & \mathbf{0} & \ddots & \mathbf{0} & \ldots & \vdots \\
\vdots & \vdots & \mathbf{0} & \alpha_{j} \boldsymbol{\Sigma}_{j} & \mathbf{0} & \vdots \\
\vdots & \vdots & \vdots & \mathbf{0} & \ddots & \mathbf{0} \\
\mathbf{0} & \ldots & \ldots & \ldots & \mathbf{0} & \alpha_{q} \boldsymbol{\Sigma}_{q}
\end{array}\right] .
$$

A inversa da matriz $\boldsymbol{\Sigma}$ pode ser escrita como $\boldsymbol{\Sigma}^{-1}=\sum_{i=1}^{q}\left(1 / \alpha_{i}\right) \mathbf{G}_{i}^{-1}$, em que $\mathbf{G}_{j}^{-1}$ denota uma matriz por blocos em que na $j$-ésima posição contém uma matriz $\boldsymbol{\Sigma}_{j}^{-1}$, isto é dado por

$$
\boldsymbol{\Sigma}^{-1}=\left[\begin{array}{cccccc}
\left(1 / \alpha_{1}\right) \boldsymbol{\Sigma}_{1}^{-1} & \mathbf{0} & \ldots & \ldots & \ldots & \mathbf{0} \\
\mathbf{0} & \left(1 / \alpha_{2}\right) \boldsymbol{\Sigma}_{2}^{-1} & \mathbf{0} & \ldots & \ldots & \vdots \\
\vdots & \mathbf{0} & \ddots & \mathbf{0} & \ldots & \vdots \\
\vdots & \vdots & \mathbf{0} & \left(1 / \alpha_{j}\right) \boldsymbol{\Sigma}_{j}^{-1} & \mathbf{0} & \vdots \\
\vdots & \vdots & \vdots & \mathbf{0} & \ddots & \mathbf{0} \\
\mathbf{0} & \cdots & \cdots & \cdots & \mathbf{0} & \left(1 / \alpha_{q}\right) \boldsymbol{\Sigma}_{q}^{-1}
\end{array}\right]
$$

A derivada parcial com respeito a $\alpha_{j}$ será dada por

$$
\frac{\partial \boldsymbol{\Sigma}}{\partial \alpha_{j}}=\frac{\partial}{\partial \alpha_{j}}\left[\sum_{i=1}^{q} \alpha_{i} \mathbf{G}_{i}\right]=\mathbf{G}_{j}
$$

Enquanto que as funções das derivadas parciais que utilizaremos nos casos particulares do Capítulo 3 ficam dadas por

$$
\boldsymbol{\Sigma}^{-1} \frac{\partial \boldsymbol{\Sigma}}{\partial \alpha_{j}}=\sum_{i=1}^{q} \frac{1}{\alpha_{i}} \mathbf{G}_{i}^{-1} \frac{\partial}{\partial \alpha_{j}}\left[\sum_{i=1}^{q} \alpha_{i} \mathbf{G}_{i}\right]=\frac{1}{\alpha_{j}} \mathbf{I}_{j}
$$

em que $\mathbf{I}_{j}$ é uma matriz por bloco com uma matriz identidade naj-ésima posição da diagonal, denotada por $\mathbf{I}_{m_{j}}$ de dimensão $\left(m_{j} \times m_{j}\right)$. Assim,

$$
\boldsymbol{\Sigma}^{-1} \frac{\partial \boldsymbol{\Sigma}}{\partial \alpha_{j}} \boldsymbol{\Sigma}^{-1}=\sum_{i=1}^{q} \frac{1}{\alpha_{i}} \mathbf{G}_{i}^{-1} \frac{\partial}{\partial \alpha_{j}}\left[\sum_{i=1}^{q} \alpha_{i} \mathbf{G}_{i}\right] \sum_{i=1}^{q} \frac{1}{\alpha_{i}} \mathbf{G}_{i}^{-1}=\frac{1}{\alpha_{j}^{2}} \mathbf{G}_{j}^{-1} .
$$


Os traços das duas últimas expressões são dadas nas seguintes equações:

$\operatorname{tr}\left(\boldsymbol{\Sigma}^{-1} \frac{\partial \boldsymbol{\Sigma}}{\partial \alpha_{j}}\right)=\operatorname{tr}\left(\frac{1}{\alpha_{j}} \mathbf{I}_{j}\right)=\frac{1}{\alpha_{j}} \operatorname{tr}\left(\mathbf{I}_{m_{j}}\right)=\frac{1}{\alpha_{j}} m_{j}$

e

$$
\begin{aligned}
\operatorname{tr}\left\{\left(\mathbf{X}^{\top} \boldsymbol{\Sigma}^{-1} \mathbf{X}\right)^{-1} \mathbf{X}^{\top} \boldsymbol{\Sigma}^{-1} \frac{\partial \boldsymbol{\Sigma}}{\partial \sigma^{2}} \boldsymbol{\Sigma}^{-1} \mathbf{X}\right\} & =\operatorname{tr}\left\{\left(\mathbf{X}^{\top} \sum_{i=1}^{q} \frac{1}{\alpha_{i}} \mathbf{G}_{i}^{-1} \mathbf{X}\right)^{-1} \mathbf{X}^{\top} \frac{1}{\alpha_{j}^{2}} \mathbf{G}_{j}^{-1} \mathbf{X}\right\} \\
& =\frac{1}{\alpha_{j}^{2}} \operatorname{tr}\left\{\left(\sum_{i=1}^{q} \frac{1}{\alpha_{i}} \mathbf{X}_{i}^{\top} \boldsymbol{\Sigma}_{i}^{-1} \mathbf{X}_{i}\right) \mathbf{X}_{j}^{\top} \boldsymbol{\Sigma}_{j}^{-1} \mathbf{X}_{j}\right\}
\end{aligned}
$$

Por último, uma forma quadrática que contém a derivada parcial de $\boldsymbol{\Sigma}$ com respeito a $\alpha_{j}$ fica dada por

$$
\begin{aligned}
(\mathbf{Y}-\mathbf{X} \boldsymbol{\beta})^{\top} \boldsymbol{\Sigma}^{-1} \frac{\partial \boldsymbol{\Sigma}}{\partial \alpha_{j}} \boldsymbol{\Sigma}^{-1}(\mathbf{Y}-\mathbf{X} \boldsymbol{\beta}) & =\frac{1}{\alpha_{j}^{2}}(\mathbf{Y}-\mathbf{X} \boldsymbol{\beta})^{\top} \mathbf{G}_{j}^{-1}(\mathbf{Y}-\mathbf{X} \boldsymbol{\beta}) \\
& =\frac{1}{\alpha_{j}^{2}}\left(\mathbf{Y}_{j}-\mathbf{X}_{j} \boldsymbol{\beta}\right)^{\top} \boldsymbol{\Sigma}_{j}^{-1}\left(\mathbf{Y}_{j}-\mathbf{X}_{j} \boldsymbol{\beta}\right)
\end{aligned}
$$

em que $\mathbf{Y}_{j}$ e $\mathbf{X}_{j}$ representam as respectivas partições do vetor $\mathbf{Y}$ e a matriz $\mathbf{X}$ da mesma forma que a matriz $\boldsymbol{\Sigma}$.

Um caso particular é quando $\boldsymbol{\Sigma}=\alpha_{1} \mathbf{G}_{1}+\alpha_{2} \mathbf{G}_{2}$, isto é quando $j$ é igual a 2 . Então, temos que a inversa de $\Sigma$ pode ser denotada como $\left(1 / \alpha_{1}\right) \mathbf{G}_{1}^{-1}+\left(1 / \alpha_{2}\right) \mathbf{G}_{2}^{-1}$ e a derivada parcial de $\boldsymbol{\Sigma}$ com respeito a $\alpha_{1}$ é dada por

$$
\frac{\partial \boldsymbol{\Sigma}}{\partial \alpha_{1}}=\frac{\partial}{\partial \alpha_{1}}\left[\alpha_{1} \mathbf{G}_{1}+\alpha_{2} \mathbf{G}_{2}\right]=\mathbf{G}_{1},
$$

da mesma forma para $\alpha_{2}$ a derivada parcial fica dada por $\partial \boldsymbol{\Sigma} / \partial \alpha_{2}=\mathbf{G}_{2}$. Assim, para $\alpha_{1}$ temos que

$\boldsymbol{\Sigma}^{-1} \frac{\partial \boldsymbol{\Sigma}}{\partial \alpha_{1}}=\frac{1}{\alpha_{1}} \mathbf{I}_{1}$

em que $\mathbf{I}_{1}$ é uma matriz por bloco com uma matriz identidade na primeira posição, de dimensão $\left(m_{1} \times m_{1}\right)$

$$
\boldsymbol{\Sigma}^{-1} \frac{\partial \boldsymbol{\Sigma}}{\partial \alpha_{1}} \boldsymbol{\Sigma}^{-1}=\frac{1}{\alpha_{1}^{2}} \mathbf{G}_{1}^{-1}
$$

Os traços das duas últimas expressões são dadas por

$\operatorname{tr}\left(\boldsymbol{\Sigma}^{-1} \frac{\partial \boldsymbol{\Sigma}}{\partial \alpha_{1}}\right)=\frac{1}{\alpha_{1}} m_{1}$

e

$\operatorname{tr}\left\{\left(\mathbf{X}^{\top} \boldsymbol{\Sigma}^{-1} \mathbf{X}\right)^{-1} \mathbf{X}^{\top} \boldsymbol{\Sigma}^{-1} \frac{\partial \boldsymbol{\Sigma}}{\partial \sigma^{2}} \boldsymbol{\Sigma}^{-1} \mathbf{X}\right\}=\frac{1}{\alpha_{1}^{2}} \operatorname{tr}\left\{\left(\frac{1}{\alpha_{1}} \mathbf{X}_{1}^{\top} \mathbf{G}_{1}^{-1} \mathbf{X}_{1}+\frac{1}{\alpha_{2}} \mathbf{X}_{2}^{\top} \mathbf{G}_{2}^{-1} \mathbf{X}_{2}\right)^{-1} \mathbf{X}_{1}^{\top} \mathbf{G}_{1}^{-1} \mathbf{X}_{1}\right\}$

Enquanto que, uma forma quadrática que contém a derivada parcial de $\boldsymbol{\Sigma}$ com respeito a $\alpha_{1}$ 
fica dada por

$$
(\mathbf{Y}-\mathbf{X} \boldsymbol{\beta})^{\top} \boldsymbol{\Sigma}^{-1} \frac{\partial \boldsymbol{\Sigma}}{\partial \alpha_{1}} \boldsymbol{\Sigma}^{-1}(\mathbf{Y}-\mathbf{X} \boldsymbol{\beta})=\frac{1}{\alpha_{1}^{2}}\left(\mathbf{Y}_{1}-\mathbf{X}_{1} \boldsymbol{\beta}\right)^{\top} \boldsymbol{\Sigma}_{1}^{-1}\left(\mathbf{Y}_{1}-\mathbf{X}_{1} \boldsymbol{\beta}\right) .
$$

Aqui consideramos a partição do vetor $\mathbf{Y}$ em $\left(\mathbf{Y}_{1}^{\top}, \mathbf{Y}_{2}^{\top}\right)^{\top}$, em que $\mathbf{Y}_{1}$ tem dimensão $\left(m_{1} \times 1\right)$ e a matriz $\mathbf{X}$ como $\left(\mathbf{X}_{1}^{\top}, \mathbf{X}_{2}^{\top}\right)^{\top}$ em que $\mathbf{X}_{1}$ tem dimensão $\left(m_{1} \times p\right)$. As derivadas com respeito a $\alpha_{2}$ têm a mesma forma.

3. Um caso em que a estrutura da matriz $\boldsymbol{\Sigma}$ é definida pela relação de dois parâmetros e dado por $\boldsymbol{\Sigma}=\sigma^{2} \mathbf{I}_{m}+\tau \mathbf{J}_{m}$, em que $\mathbf{J}_{m}$ é uma matriz cujos elementos são 1 e pode ser escrita como $\mathbf{1 1}^{\top}$, em que $\mathbf{1}$ denota um vetor de dimensão $(m \times 1)$ compostos por 1. Portanto, podemos escrever a matriz $\boldsymbol{\Sigma}$ na forma

$$
\boldsymbol{\Sigma}=\sigma^{2} \mathbf{I}_{m}+\tau \mathbf{J}_{m}=\left[\begin{array}{cccccc}
\sigma^{2}+\tau & \tau & \tau & \tau & \tau & \tau \\
\tau & \sigma^{2}+\tau & \tau & \ldots & \cdots & \vdots \\
\vdots & \tau & \ddots & \tau & \cdots & \vdots \\
\vdots & \vdots & \tau & \sigma^{2}+\tau & \tau & \vdots \\
\vdots & \vdots & \vdots & \tau & \ddots & \tau \\
\tau & \cdots & \cdots & \cdots & \tau & \sigma^{2}+\tau
\end{array}\right] .
$$

A matriz inversa de $\boldsymbol{\Sigma}$ fica dada por

$$
\boldsymbol{\Sigma}^{-1}=\frac{1}{\sigma^{2}}\left(\mathbf{I}_{m}-\frac{\tau}{\sigma^{2}+m \tau} \mathbf{J}_{m}\right)=\frac{1}{\sigma^{2}\left(\sigma^{2}+m \tau\right)}\left\{\left(\sigma^{2}+m \tau\right) \mathbf{I}_{m}-\tau \mathbf{J}_{m}\right\}
$$

para $\sigma^{2} \neq 0$ e $\sigma^{2} \neq m \tau$. O determinante de $\boldsymbol{\Sigma}$ fica dado por

$$
|\boldsymbol{\Sigma}|=\alpha^{m-1}(\alpha+m \tau)
$$

Consideraremos que a matriz $\mathbf{J}_{m}$ é uma matriz simétrica, isto é $\mathbf{J}_{m}^{\top}=\mathbf{J}_{m}$, e que $\mathbf{J}_{m} \mathbf{J}_{m}=$ $\mathbf{1 1}^{\top} \mathbf{1} \mathbf{1}^{\top}=m \mathbf{J}_{m}$.

A derivada parcial de $\boldsymbol{\Sigma}$ com respeito a $\sigma^{2}$ fica dada por

$$
\frac{\partial \boldsymbol{\Sigma}}{\partial \sigma^{2}}=\mathbf{I}_{m}
$$

Duas funções da derivada parcial com respeito a $\sigma^{2}$ são dadas por

$$
\boldsymbol{\Sigma}^{-1} \frac{\partial \boldsymbol{\Sigma}}{\partial \sigma^{2}}=\frac{1}{\sigma^{2}}\left(\mathbf{I}_{m}-\frac{\tau}{\sigma^{2}+m \tau} \mathbf{J}_{m}\right) \mathbf{I}_{m}=\frac{1}{\sigma^{2}}\left(\mathbf{I}_{m}-\frac{\tau}{\sigma^{2}+m \tau} \mathbf{J}_{m}\right)
$$

e

$$
\begin{aligned}
\boldsymbol{\Sigma}^{-1} \frac{\partial \boldsymbol{\Sigma}}{\partial \sigma^{2}} \boldsymbol{\Sigma}^{-1} & =\frac{1}{\sigma^{2}}\left(\mathbf{I}_{m}-\frac{\tau}{\sigma^{2}+m \tau} \mathbf{J}_{m}\right) \frac{1}{\sigma^{2}}\left(\mathbf{I}_{m}-\frac{\tau}{\sigma^{2}+m \tau} \mathbf{J}_{m}\right) \\
& =\frac{1}{\left(\sigma^{2}\right)^{2}}\left\{\mathbf{I}_{m}-2 \frac{\tau}{\sigma^{2}+m \tau} \mathbf{J}_{m}+m\left(\frac{\tau}{\sigma^{2}+m \tau}\right)^{2} \mathbf{J}_{m}\right\} \\
& =\left\{\frac{1}{\left(\sigma^{2}\right)\left(\sigma^{2}+m \tau\right)}\right\}^{2}\left\{\left(\sigma^{2}+m \tau\right)^{2} \mathbf{I}_{m}-\left(2 \tau \sigma^{2}+m \tau^{2}\right) \mathbf{J}_{m}\right\} .
\end{aligned}
$$


Os traços de duas funções da derivada parcial de $\boldsymbol{\Sigma}$ com respeito a $\sigma^{2}$ ficam dados por

$$
\begin{aligned}
\operatorname{tr}\left(\boldsymbol{\Sigma}^{-1} \frac{\partial \boldsymbol{\Sigma}}{\partial \sigma^{2}}\right)=\frac{1}{\sigma^{2}} \operatorname{tr}\left(\mathbf{I}_{m}-\frac{\tau}{\sigma^{2}+m \tau} \mathbf{J}_{m}\right) & =\frac{1}{\sigma^{2}}\left\{\operatorname{tr}\left(\mathbf{I}_{m}\right)-\frac{\tau}{\sigma^{2}+m \tau} \operatorname{tr}\left(\mathbf{J}_{m}\right)\right\} \\
& =\frac{1}{\sigma^{2}}\left\{m-\frac{\tau}{\sigma^{2}+m \tau} m\right\} \\
& =\frac{m}{\sigma^{2}}\left\{\frac{\sigma^{2}+(m-1) \tau}{\sigma^{2}+m \tau}\right\}
\end{aligned}
$$

e

$$
\begin{aligned}
& \operatorname{tr}\left\{\left(\mathbf{X}^{\top} \boldsymbol{\Sigma}^{-1} \mathbf{X}\right)^{-1} \mathbf{X}^{\top} \boldsymbol{\Sigma}^{-1} \frac{\partial \boldsymbol{\Sigma}}{\partial \sigma^{2}} \boldsymbol{\Sigma}^{-1} \mathbf{X}\right\} \\
& =c(\sigma, \tau) \operatorname{tr}\left[\left\{\left(\sigma^{2}+m \tau\right) \mathbf{X}^{\top} \mathbf{X}-\tau \mathbf{X}^{\top} \mathbf{J}_{m} \mathbf{X}\right\}^{-1}\left\{\left(\sigma^{2}+m \tau\right)^{2} \mathbf{X}^{\top} \mathbf{X}-\left(2 \tau \sigma^{2}+m \tau^{2}\right) \mathbf{X}^{\top} \mathbf{J}_{m} \mathbf{X}\right\}\right]
\end{aligned}
$$

em que $c(\sigma, \tau)$ é dado por $\left(\sigma^{2}\right)\left(\sigma^{2}+m \tau\right)^{-1}$.

Enquanto que, uma forma quadrática que contém a derivada parcial de $\boldsymbol{\Sigma}$ com respeito a $\sigma^{2}$ fica dada por

$$
\begin{aligned}
(\mathbf{Y}-\mathbf{X} \boldsymbol{\beta})^{\top} \boldsymbol{\Sigma}^{-1} \frac{\partial \boldsymbol{\Sigma}}{\partial \sigma^{2}} \boldsymbol{\Sigma}^{-1}(\mathbf{Y}-\mathbf{X} \boldsymbol{\beta}) & =\frac{1}{\sigma^{2}}(\mathbf{Y}-\mathbf{X} \boldsymbol{\beta})^{\top} \mathbf{I}_{m}(\mathbf{Y}-\mathbf{X} \boldsymbol{\beta}) \\
& -c_{2}(\sigma, \tau)(\mathbf{Y}-\mathbf{X} \boldsymbol{\beta})^{\top} \mathbf{J}_{m}(\mathbf{Y}-\mathbf{X} \boldsymbol{\beta}) \\
& =\frac{1}{\sigma^{2}} \sum_{i=1}^{n}\left(Y_{i}-\mathbf{X}_{i} \boldsymbol{\beta}\right)^{2}-c_{2}(\sigma, \tau)\left\{\sum_{i=1}^{n}\left(Y_{i}-\mathbf{X}_{i} \boldsymbol{\beta}\right)\right\}^{2} \\
& =\frac{1}{\sigma^{2}} S_{Y Y}-c_{2}(\sigma, \tau) S_{Y}^{2},
\end{aligned}
$$

em que $c_{2}(\sigma, \tau)$ é dado por $\frac{\left(2 \tau \sigma^{2}+m \tau^{2}\right)}{\left\{\sigma^{2}\left(\sigma^{2}+m \tau\right)\right\}^{2}}$.

Já a derivada parcial de $\boldsymbol{\Sigma}$ com respeito a $\tau$ fica dada por

$$
\frac{\partial \boldsymbol{\Sigma}}{\partial \tau}=\mathbf{J}_{m}
$$

Duas funções da derivada parcial de $\boldsymbol{\Sigma}$ com respeito a $\tau$ são dadas por

$$
\begin{aligned}
\boldsymbol{\Sigma}^{-1} \frac{\partial \boldsymbol{\Sigma}}{\partial \tau} & =\frac{1}{\sigma^{2}}\left(\mathbf{I}_{m}-\frac{\tau}{\sigma^{2}+m \tau} \mathbf{J}_{m}\right) \mathbf{J}_{m}=\frac{1}{\sigma^{2}}\left(\mathbf{J}_{m}-\frac{\tau}{\sigma^{2}+m \tau} \mathbf{J}_{m} \mathbf{J}_{m}\right) \\
& =\frac{1}{\sigma^{2}}\left(1-\frac{\tau m}{\sigma^{2}+m \tau}\right) \mathbf{J}_{m}=\frac{1}{\sigma^{2}}\left(\frac{\sigma^{2}}{\sigma^{2}+m \tau}\right) \mathbf{J}_{m} \\
& =\frac{1}{\sigma^{2}+m \tau} \mathbf{J}_{m}
\end{aligned}
$$


e

$$
\begin{aligned}
\boldsymbol{\Sigma}^{-1} \frac{\partial \boldsymbol{\Sigma}}{\partial \tau} \boldsymbol{\Sigma}^{-1} & =\left(\frac{1}{\sigma^{2}+m \tau}\right) \mathbf{J}_{m} \frac{1}{\sigma^{2}}\left(\mathbf{I}_{m}-\frac{\tau}{\sigma^{2}+m \tau} \mathbf{J}_{m}\right) \\
& =\left(\frac{1}{\sigma^{2}+m \tau}\right)\left(\frac{1}{\sigma^{2}+m \tau}\right) \mathbf{J}_{m} \\
& =\left(\frac{1}{\sigma^{2}+m \tau}\right)^{2} \mathbf{J}_{m}
\end{aligned}
$$

Os traço das duas últimas expressões com respeito a $\tau$ ficam dados por

$\operatorname{tr}\left(\boldsymbol{\Sigma}^{-1} \frac{\partial \boldsymbol{\Sigma}}{\partial \tau}\right)=\operatorname{tr}\left\{\frac{1}{\sigma^{2}+m \tau} \mathbf{J}_{m}\right\}=\frac{1}{\sigma^{2}+m \tau} \operatorname{tr}\left(\mathbf{J}_{m}\right)=\frac{m}{\sigma^{2}+m \tau}$

e

$\operatorname{tr}\left\{\left(\mathbf{X}^{\top} \boldsymbol{\Sigma}^{-1} \mathbf{X}\right)^{-1} \mathbf{X}^{\top} \boldsymbol{\Sigma}^{-1} \frac{\partial \boldsymbol{\Sigma}}{\partial \tau} \boldsymbol{\Sigma}^{-1} \mathbf{X}\right\}=c_{3}(\sigma, \tau) \operatorname{tr}\left[\left\{\left(\sigma^{2}+m \tau\right) \mathbf{X}^{\top} \mathbf{X}-\tau \mathbf{X}^{\top} \mathbf{J}_{m} \mathbf{X}^{-1} \mathbf{X}^{\top} \mathbf{J}_{m} \mathbf{X}\right.\right.$

em que $c_{3}(\sigma, \tau)$ é dado por $\frac{\sigma^{2}}{\sigma^{2}+m \tau}$.

Por último, a forma quadrática que contém a derivada parcial de $\boldsymbol{\Sigma}$ com respeito a $\tau$ fica dada por

$$
\begin{aligned}
(\mathbf{Y}-\mathbf{X} \boldsymbol{\beta})^{\top} \boldsymbol{\Sigma}^{-1} \frac{\partial \boldsymbol{\Sigma}}{\partial \tau} \boldsymbol{\Sigma}^{-1}(\mathbf{Y}-\mathbf{X} \boldsymbol{\beta}) & =\left(\frac{1}{\sigma^{2}+m \tau}\right)^{2}(\mathbf{Y}-\mathbf{X} \boldsymbol{\beta})^{\top} \mathbf{J}_{m}(\mathbf{Y}-\mathbf{X} \boldsymbol{\beta}) \\
& =\left(\frac{1}{\sigma^{2}+m \tau}\right)^{2}\left\{\sum_{i=1}^{n}\left(Y_{i}-\mathbf{X}_{i} \boldsymbol{\beta}\right)\right\}^{2} \\
& =\left(\frac{1}{\sigma^{2}+m \tau}\right)^{2} S_{Y}^{2}
\end{aligned}
$$

4. Quando a matriz $\boldsymbol{\Sigma}$ fica dada por $\sigma^{2} \mathbf{R}, \mathbf{R}$ fica dada por

$$
\mathbf{R}=\left[\begin{array}{cccccc}
1 & \rho & \rho^{2} & \ldots & \rho^{m-2} & \rho^{m-1} \\
\rho & 1 & \rho & \ldots & \rho^{m-3} & \rho^{m-2} \\
\rho^{2} & \rho & 1 & \ldots & \rho^{m-4} & \rho^{m-3} \\
\vdots & \vdots & \vdots & \ddots & \vdots & \vdots \\
\rho^{m-2} & \rho^{m-3} & \rho^{m-4} & \ldots & 1 & \rho \\
\rho^{m-1} & \rho^{m-2} & \rho^{m-3} & \ldots & \rho & 1
\end{array}\right]
$$

A matriz inversa de $\mathbf{R}$ tem a seguinte estrutura:

$$
\mathbf{R}^{-1}=\frac{1}{1-\rho^{2}}\left[\begin{array}{cccccc}
1 & -\rho & 0 & \cdots & 0 & 0 \\
-\rho & 1+\rho^{2} & -\rho & \cdots & 0 & 0 \\
0 & -\rho & 1+\rho^{2} & \cdots & 0 & 0 \\
\vdots & \vdots & \vdots & \ddots & \vdots & \vdots \\
0 & 0 & 0 & \cdots & 1+\rho^{2} & -\rho \\
0 & 0 & 0 & \cdots & -\rho & 1
\end{array}\right] .
$$

A matriz tem três tipos de elementos, $r_{1,1}=r_{m, m}=1 /\left(1-\rho^{2}\right), r_{i, i+1}=r_{i, i-1}=-\rho /\left(1-\rho^{2}\right)$ e $r_{i, i}=\left(1+\rho^{2}\right) /\left(1-\rho^{2}\right)$, para $i=2, \ldots, n-1$. Os demais elementos são zeros. A derivada 
parcial de $\mathbf{R}$ com respeito a $\rho$ fica dada por

$$
\frac{\partial \mathbf{R}}{\partial \rho}=\left[\begin{array}{cccccc}
0 & 1 & 2 \rho & \ldots & (m-2) \rho^{m-3} & (m-1) \rho^{m-2} \\
1 & 0 & 1 & \ldots & (m-3) \rho^{m-4} & (m-2) \rho^{m-3} \\
2 \rho & 1 & 0 & \ldots & (m-4) \rho^{m-5} & (m-3) \rho^{m-4} \\
\vdots & \vdots & \vdots & \ddots & \vdots & \vdots \\
(m-2) \rho^{m-3} & (m-3) \rho^{m-4} & (m-4) \rho^{m-5} & \ldots & 0 & 1 \\
(m-1) \rho^{m-2} & (m-2) \rho^{m-3} & (m-3) \rho^{m-4} & \ldots & 1 & 0
\end{array}\right] .
$$

Algumas funções úteis da derivada parcial com respeito a $\rho$, ficam dadas por $\mathbf{R}^{-1} \frac{\partial \mathbf{R}}{\partial \rho}=$

$\frac{1}{1-\rho^{2}}\left[\begin{array}{cccccc}-\rho & 1 & \rho & \ldots & \rho^{m-1} & \rho^{m-2} \\ 1-\rho^{2} & -2 \rho & 1-\rho^{2} & \ldots & \rho^{m-4}-\rho^{m-2} & \rho^{m-3}-\rho^{m-1} \\ \rho-\rho^{3} & 1-\rho^{2} & -2 \rho & \ldots & \rho^{m-5}-\rho^{m-3} & \rho^{m-4}-\rho^{m-2} \\ \vdots & \vdots & \vdots & \ddots & \vdots & \vdots \\ \rho^{m-3}-\rho^{m-1} & \rho^{m-4}-\rho^{m-2} & \rho^{m-5}-\rho^{m-3} & \ldots & -2 \rho & 1-\rho^{2} \\ \rho^{m-2} & \rho^{m-3} & \ldots & \ldots & 1 & -\rho\end{array}\right]$

$\mathrm{e}$

$$
\mathbf{R}^{-1} \frac{\partial \mathbf{R}}{\partial \rho} \mathbf{R}^{-1}=-\frac{1}{\left(1-\rho^{2}\right)^{2}}\left[\begin{array}{cccccc}
-2 \rho & 1+\rho^{2} & 0 & \cdots & 0 & 0 \\
1+\rho^{2} & 4 \rho & 1+\rho^{2} & \cdots & 0 & 0 \\
0 & 1+\rho^{2} & 4 \rho & \cdots & 0 & 0 \\
\vdots & \vdots & \vdots & \ddots & \vdots & \vdots \\
0 & 0 & 0 & \cdots & 4 \rho & 1+\rho^{2} \\
0 & 0 & \cdots & \cdots & 1+\rho^{2} & -2 \rho
\end{array}\right] .
$$

Então, temos que o traço de $\mathbf{R}^{-1} \frac{\partial \mathbf{R}}{\partial \rho}$ fica dado por

$$
\operatorname{tr}\left(\mathbf{R}^{-1} \frac{\partial \mathbf{R}}{\partial \rho}\right)=\frac{1}{1-\rho^{2}}(-2 \rho(m-1))=\frac{-2 \rho(m-1)}{1-\rho^{2}} .
$$

Seja a matriz $\mathbf{X}$ particionada em vetores $\mathbf{X}_{i}$ com $i=1, \ldots, m$ de dimensão $(1 \times p)$, então temos que

$$
\begin{aligned}
\mathbf{X}^{\top} \mathbf{R}^{-1} \mathbf{X} & =\frac{1}{1-\rho^{2}}\left[\begin{array}{llll}
(1-\rho) \mathbf{X}_{1}^{\top} & (1-\rho)^{2} \mathbf{X}_{2}^{\top} & \ldots & (1-\rho) \mathbf{X}_{m}^{\top}
\end{array}\right]\left[\begin{array}{c}
\mathbf{X}_{1} \\
\mathbf{X}_{2} \\
\vdots \\
\mathbf{X}_{m-1} \\
\mathbf{X}_{m}
\end{array}\right] \\
& =\frac{1}{(1-\rho)(1+\rho)}\left((1-\rho) \mathbf{X}_{1}^{\top} \mathbf{X}_{1}+(1-\rho)^{2} \mathbf{X}_{2}^{\top} \mathbf{X}_{2}+\ldots+(1-\rho) \mathbf{X}_{m}^{\top} \mathbf{X}_{m}\right) \\
& =\frac{(1-\rho)}{(1-\rho)(1+\rho)}\left(\mathbf{X}_{1}^{\top} \mathbf{X}_{1}+(1-\rho) \mathbf{X}_{2}^{\top} \mathbf{X}_{2}+\ldots+\mathbf{X}_{m}^{\top} \mathbf{X}_{m}\right) \\
& =\frac{1}{(1+\rho)}\left(\sum_{i=1}^{m} \mathbf{X}_{i}^{\top} \mathbf{X}_{i}-\rho \sum_{i=2}^{m-1} \mathbf{X}_{i}^{\top} \mathbf{X}_{i}\right) .
\end{aligned}
$$


Agora considerando os elementos do vetor $\mathbf{Y}$, temos que

$\mathbf{X}^{\top} \mathbf{R}^{-1} \mathbf{Y}$

$$
\begin{aligned}
& =\frac{1}{1-\rho^{2}}\left[\begin{array}{lllll}
(1-\rho) \mathbf{X}_{1}^{\top} & (1-\rho)^{2} \mathbf{X}_{2}^{\top} & \ldots & (1-\rho)^{2} \mathbf{X}_{m-1}^{\top} & (1-\rho) \mathbf{X}_{m}^{\top}
\end{array}\right]\left[\begin{array}{c}
Y_{1} \\
Y_{2} \\
\vdots \\
Y_{m-1} \\
Y_{m}
\end{array}\right] \\
& =\frac{1}{(1+\rho)}\left(\sum_{i=1}^{m} \mathbf{X}_{i}^{\top} Y_{i}-\rho \sum_{i=2}^{m-1} \mathbf{X}_{i}^{\top} Y_{i}\right) .
\end{aligned}
$$

Enquanto que, a matriz $\mathbf{X}^{\top} \mathbf{R}^{-1} \frac{\partial \mathbf{R}}{\partial \rho} \mathbf{R}^{-1} \mathbf{X}$ fica dada por

$$
\begin{aligned}
& \left(\mathbf{X}^{\top} \mathbf{R}^{-1} \frac{\partial \mathbf{R}}{\partial \rho} \mathbf{R}^{-1} \mathbf{X}\right) \\
& =\frac{1}{\left(1-\rho^{2}\right)^{2}}\left[\begin{array}{llll}
(1-\rho)^{2} \mathbf{X}_{1}^{\top} & 2(1+\rho)^{2} \mathbf{X}_{2}^{\top} & \ldots & (1-\rho)^{2} \mathbf{X}_{m}^{\top}
\end{array}\right]\left[\begin{array}{c}
\mathbf{X}_{1} \\
\mathbf{X}_{2} \\
\vdots \\
\mathbf{X}_{m-1} \\
\mathbf{X}_{m}
\end{array}\right] \\
& =\frac{\mathbf{X}_{1}^{\top} \mathbf{X}_{1}+\mathbf{X}_{m}^{\top} \mathbf{X}_{m}}{(1+\rho)^{2}}+\frac{2 \sum_{i=2}^{m-1} \mathbf{X}_{i}^{\top} \mathbf{X}_{i}}{(1-\rho)^{2}} .
\end{aligned}
$$

Como a função traço de uma matriz é a soma dos elementos da diagonal principal da matriz, o traço de um escalar é igual ao escalar. Assim, neste caso, o traço de $\left(\mathbf{X}^{\top} \mathbf{R}^{-1} \mathbf{X}\right)^{-1} \mathbf{X}^{\top}$ $\mathbf{R}^{-1} \frac{\partial \mathbf{R}}{\partial \rho} \mathbf{R}^{-1} \mathbf{X}$ fica dado por

$$
\begin{aligned}
& \left(\mathbf{X}^{\top} \mathbf{R}^{-1} \mathbf{X}\right)^{-1} \mathbf{X}^{\top} \mathbf{R}^{-1} \frac{\partial \mathbf{R}}{\partial \rho} \mathbf{R}^{-1} \mathbf{X} \\
& =\frac{\mathbf{X}_{1}^{\top} \mathbf{X}_{1}+\mathbf{X}_{m}^{\top} \mathbf{X}_{m}}{(1+\rho)\left(\sum_{i=1}^{m} \mathbf{X}_{i}^{\top} \mathbf{X}_{i}-\rho \sum_{i=2}^{m-1} \mathbf{X}_{i}^{\top} \mathbf{X}_{i}\right)}+\frac{2(1+\rho) \sum_{i=2}^{m-1} \mathbf{X}_{i}^{\top} \mathbf{X}_{i}}{(1-\rho)^{2}\left(\sum_{i=1}^{m} \mathbf{X}_{i}^{\top} \mathbf{X}_{i}-\rho \sum_{i=2}^{m-1} \mathbf{X}_{i}^{\top} \mathbf{X}_{i}\right)} \\
& =\frac{\mathbf{X}_{1}^{\top} \mathbf{X}_{1}+\mathbf{X}_{m}^{\top} \mathbf{X}_{m}}{(1+\rho)\left(\sum_{i=1}^{m} \mathbf{X}_{i}^{\top} \mathbf{X}_{i}-\rho \sum_{i=2}^{m-1} \mathbf{X}_{i}^{\top} \mathbf{X}_{i}\right)}+\frac{2(1+\rho) \sum_{i=2}^{m-1} \mathbf{X}_{i}^{\top} \mathbf{X}_{i}}{(1-\rho)^{2}\left(\sum_{i=1}^{m} \mathbf{X}_{i}^{\top} \mathbf{X}_{i}-\rho \sum_{i=2}^{m-1} \mathbf{X}_{i}^{\top} \mathbf{X}_{i}\right)}
\end{aligned}
$$

Além disso, a forma quadrática $(\mathbf{Y}-\mathbf{X} \boldsymbol{\beta})^{\top} \boldsymbol{\Sigma}^{-1} \frac{\partial \boldsymbol{\Sigma}}{\partial \rho} \boldsymbol{\Sigma}^{-1}(\mathbf{Y}-\mathbf{X} \boldsymbol{\beta})$ fica dada por

$$
\begin{aligned}
(\mathbf{Y}-\mathbf{X} \boldsymbol{\beta})^{\top} \boldsymbol{\Sigma}^{-1} \frac{\partial \boldsymbol{\Sigma}}{\partial \rho} \boldsymbol{\Sigma}^{-1}(\mathbf{Y}-\mathbf{X} \boldsymbol{\beta}) & =\frac{1}{\left(1-\rho^{2}\right)^{2}}\left[(1-\rho)^{2}\left\{\left(Y_{1}-\mathbf{X}_{1} \boldsymbol{\beta}\right)^{2}+\left(Y_{m}-\mathbf{X}_{m} \boldsymbol{\beta}\right)^{2}\right\}\right. \\
& \left.+2(1+\rho)^{2} \sum_{i=2}^{m-1}\left(Y_{i}-\mathbf{X}_{i} \boldsymbol{\beta}\right)^{2}\right]
\end{aligned}
$$

Um caso particular é considerar a matriz $\mathbf{X}$ como um vetor de uns com dimensão $(m \times 1)$, 
então, considerando a equação (B.23) temos que

$$
\begin{aligned}
& \left(\mathbf{X}^{\top} \mathbf{R}^{-1} \mathbf{X}\right)=\mathbf{1}^{\top} \mathbf{R}^{-1} \mathbf{1} \\
& =\frac{1}{1-\rho^{2}}\left[\begin{array}{llll}
1-\rho & -2 \rho+1+\rho^{2} & \ldots & 1-\rho
\end{array}\right]\left[\begin{array}{c}
1 \\
1 \\
\vdots \\
1 \\
1
\end{array}\right] \\
& =\frac{m-(m-2) \rho}{1+\rho},
\end{aligned}
$$

e da equação (B.24) temos

$$
\begin{aligned}
& \left(\mathbf{X}^{\top} \mathbf{R}^{-1} \mathbf{Y}\right)=\mathbf{1}^{\top} \mathbf{R}^{-1} \mathbf{Y} \\
& =\frac{1}{1-\rho^{2}}\left[\begin{array}{llll}
1-\rho & -2 \rho+1+\rho^{2} & \ldots & 1-\rho
\end{array}\right]\left[\begin{array}{c}
Y_{1} \\
Y_{2} \\
\vdots \\
Y_{m-1} \\
Y_{m}
\end{array}\right] \\
& =\frac{\sum_{i=1}^{m} Y_{i}-\rho \sum_{i=2}^{m-1} Y_{i}}{1+\rho} .
\end{aligned}
$$

Enquanto que, a matriz $\mathbf{X}^{\top} \mathbf{R}^{-1} \frac{\partial \mathbf{R}}{\partial \rho} \mathbf{R}^{-1} \mathbf{X}$ fica dada por

$\mathbf{1}^{\top} \mathbf{R}^{-1} \frac{\partial \mathbf{R}}{\partial \rho} \mathbf{R}^{-1} \mathbf{1}$

$$
\begin{aligned}
& =\frac{1}{\left(1-\rho^{2}\right)^{2}}\left[\begin{array}{llll}
\rho^{2}-2 \rho+1 & 2\left(1+\rho^{2}\right)+4 \rho & \ldots & \rho^{2}-2 \rho+1
\end{array}\right]\left[\begin{array}{c}
1 \\
1 \\
\vdots \\
1 \\
1
\end{array}\right] \\
& =\frac{2}{(1+\rho)^{2}}+\frac{2(m-2)}{(1-\rho)^{2}} .
\end{aligned}
$$

Análogo à equação (B.25)

$$
\begin{aligned}
& \left(\mathbf{1}^{\top} \mathbf{R}^{-1} \mathbf{1}\right)^{-1} \mathbf{1}^{\top} \mathbf{R}^{-1} \frac{\partial \mathbf{R}}{\partial \rho} \mathbf{R}^{-1} \mathbf{1} \\
& =\left\{\frac{m-(m-2) \rho}{1+\rho}\right\}^{-1}\left\{\frac{2}{(1+\rho)^{2}}+\frac{2(m-2)}{(1-\rho)^{2}}\right\} .
\end{aligned}
$$

Finalmente, como o vetor $\mathbf{X}$ só tem uma coluna, isso é que $p=1$ então o parâmetro $\boldsymbol{\beta}$ é escalar. Assim a forma quadrática $(\mathbf{Y}-\mathbf{X} \boldsymbol{\beta})^{\top} \boldsymbol{\Sigma}^{-1} \frac{\partial \boldsymbol{\Sigma}}{\partial \rho} \boldsymbol{\Sigma}^{-1}(\mathbf{Y}-\mathbf{X} \boldsymbol{\beta})$ fica dada por

$$
\begin{aligned}
(\mathbf{Y}-\mathbf{1} \beta)^{\top} \mathbf{R}^{-1} \frac{\partial \mathbf{R}}{\partial \rho} \mathbf{R}^{-1}(\mathbf{Y}-\mathbf{1} \beta) & =\frac{1}{\left(1-\rho^{2}\right)^{2}}\left[(1-\rho)^{2}\left\{\left(Y_{1}-\beta\right)^{2}+\left(Y_{m}-\beta\right)^{2}\right\}\right. \\
& \left.+2(1+\rho)^{2} \sum_{i=2}^{m-1}\left(Y_{i}-\beta\right)^{2}\right] .
\end{aligned}
$$




\section{Apêndice C}

\section{Distribuição elíptica}

Neste apêndice apresentamos a definição da distribuição elíptica e algumas propriedades que serão importantes para desenvolver os métodos de estimação dos parâmetros dessa distribuição. Os resultados apresentados neste apêndice são baseados na Tese de Doutorado de Arellano (1994).

\section{C.1 Definição da família elíptica}

Definição C.1.1 Consideremos um vetor aleatório $\mathbf{Y}$ de dimensão $(m \times 1)$ com distribuição elíptica simétrica com parâmetros $\boldsymbol{\mu}_{(m \times 1)}$ e $\Sigma_{(m \times m)}$, se

$$
\mathbf{Y} \stackrel{d}{=} \boldsymbol{\mu}+\mathbf{A}^{\top} \mathbf{S}
$$

em que $\mathbf{A}$ é uma matriz $(k \times m)$, tal que $\mathbf{A}^{\top} \mathbf{A}=\boldsymbol{\Sigma}$ e o posto de $\boldsymbol{\Sigma}$ é $k, k \leq m$, e $\mathbf{S}$ é um vetor aleatório com distribuição esférica de dimensão $k$ e função escalar $\phi$. Então, $\mathbf{Y}$ tem distribuição eliptica e escreve-se como $\mathbf{Y} \sim E l_{m}(\boldsymbol{\mu}, \boldsymbol{\Sigma}, \phi)$.

A função $\phi$ é determinada pela seguinte relação. Seja $\psi(t)=\mathrm{E}\left(e^{i t^{T} y}\right)$ a função característica de $\mathbf{Y}$, que tem forma dada por

$$
\psi(t)=e^{i t^{T} \boldsymbol{\mu}} \phi\left(t^{\top} \boldsymbol{\Sigma} t\right) .
$$

Uma condição necessária para que a função de densidade de $\mathbf{Y}$ exista é que o posto de $\boldsymbol{\Sigma}$ seja $m$, isto é, que $\boldsymbol{\Sigma}>0$. Então, para $k=m$, temos que $\mathbf{A}$ é uma matriz quadrada e o posto de $\boldsymbol{\Sigma}$ é completo, isto é equivalente a dizer que $\boldsymbol{\Sigma}$ é não singular. Assim, podemos escrever $\mathbf{I}_{m}=\mathbf{A}^{\top} \boldsymbol{\Sigma}^{-1} \mathbf{A}$ e a representação estocástica de $\mathbf{Y}$ fica dada por

$$
\mathbf{Y} \stackrel{d}{=} \boldsymbol{\mu}+r \mathbf{A}^{\top} \mathbf{s} \quad r \geq 0
$$

em que $r$ é uma variável aleatória independente de $\mathbf{s}$. O vetor $\mathbf{s}$ tem distribuição uniforme no espaço esférico unitário em $\mathbf{R}^{m}$ e é a dimensão deste vetor que determina a dimensão de $\mathbf{Y}$.

Então, a função de densidade de $\mathbf{Y}$ existe e pode ser denotada por $\mathbf{Y} \sim E l_{m}(\boldsymbol{\mu}, \boldsymbol{\Sigma}, g)$ e sua forma é dada por

$$
f_{\mathbf{Y}}(\mathbf{y} ; \boldsymbol{\mu}, \boldsymbol{\Sigma})=|\boldsymbol{\Sigma}|^{-1 / 2} g\left\{(\mathbf{y}-\boldsymbol{\mu})^{\top} \boldsymbol{\Sigma}^{-1}(\mathbf{y}-\boldsymbol{\mu})\right\}
$$

em que $g(\cdot)$ é uma função contínua que define a densidade de $\mathbf{Y}$ e que denominaremos função geradora da densidade de $\mathbf{Y}$.

\section{C.2 Propriedades da família elíptica}

Vamos considerar o vetor aleatório $\mathbf{Y}$ com distribuição $E l_{m}(\boldsymbol{\mu}, \boldsymbol{\Sigma}, \phi)$, em que $\boldsymbol{\mu}=\mathbf{X} \boldsymbol{\beta} \in \mathbb{R}$ e $\boldsymbol{\Sigma} \geq 0$ e tem as seguintes propriedades: 
1. Seja $\mathbf{B}_{(m \times n)}$ uma matriz e $\mathbf{V}_{(n \times 1)}$ um vetor, então,

$$
\mathbf{V}+\mathbf{B}^{\top} \mathbf{Y} \sim E l_{n}\left(\mathbf{V}+\mathbf{B}^{\top} \boldsymbol{\mu}, \mathbf{B}^{\top} \boldsymbol{\Sigma} \mathbf{B}, \phi\right) .
$$

Podemos ver que a distribuição de uma transformação linear de $\mathbf{Y}$ mantém a distribuição elíptica mas os parâmetros mudam.

2. Se consideramos a seguinte partição de $\mathbf{Y}, \boldsymbol{\mu}$ e $\boldsymbol{\Sigma}$ :

$$
\mathbf{Y}=\left[\begin{array}{l}
\mathbf{Y}_{1} \\
\mathbf{Y}_{2}
\end{array}\right], \quad \boldsymbol{\mu}=\left[\begin{array}{l}
\boldsymbol{\mu}_{1} \\
\boldsymbol{\mu}_{2}
\end{array}\right] \quad \boldsymbol{\Sigma}=\left[\begin{array}{ll}
\boldsymbol{\Sigma}_{11} & \boldsymbol{\Sigma}_{12} \\
\boldsymbol{\Sigma}_{21} & \boldsymbol{\Sigma}_{22}
\end{array}\right]
$$

Então, as distribuições marginais também têm distribuição elíptica,

$$
\begin{aligned}
& \mathbf{Y}_{1} \sim E l_{n}\left(\boldsymbol{\mu}_{1}, \boldsymbol{\Sigma}_{11}, \phi\right) \\
& \mathbf{Y}_{2} \sim E l_{m-n}\left(\boldsymbol{\mu}_{2}, \boldsymbol{\Sigma}_{22}, \phi\right) .
\end{aligned}
$$

Agora, se consideramos que a função de densidade de $\mathbf{Y}$ existe, a função geradora de densidade $g(\cdot)$ pode ser especificada. Então, as funções de densidade das marginais de $\mathbf{Y}_{1}$ e $\mathbf{Y}_{2}$ terão distribuição elíptica mas as funções geradoras de densidades podem não ser iguais a $g(\cdot)$, portanto as denotaremos como $g_{1}(\cdot)$ e $g_{2}(\cdot)$, respectivamente. Assim, as distribuições marginais podem ser denotadas como

$$
\begin{aligned}
& \mathbf{Y}_{1} \sim E l_{n}\left(\boldsymbol{\mu}_{1}, \boldsymbol{\Sigma}_{11}, g_{1}\right) \\
& \mathbf{Y}_{2} \sim E l_{m-n}\left(\boldsymbol{\mu}_{2}, \boldsymbol{\Sigma}_{22}, g_{2}\right) .
\end{aligned}
$$

Para obter a função de densidade de uma das variáveis marginais, por exemplo, a variável $\mathbf{Y}_{2}$, teremos que integrar a função de densidade conjunta com respeito a $\mathbf{Y}_{1}$, então

$$
f_{\mathbf{Y}_{2}}\left(\mathbf{y}_{2}\right)=\int_{R^{n}}|\boldsymbol{\Sigma}|^{-1 / 2} g\left\{(\mathbf{Y}-\boldsymbol{\mu})^{\top} \boldsymbol{\Sigma}^{-1}(\mathbf{Y}-\boldsymbol{\mu})\right\} d \mathbf{y}_{1} .
$$

Consideraremos a seguinte propriedade do determinante para a partição de $\boldsymbol{\Sigma}$ de (C.2):

$$
\begin{aligned}
|\boldsymbol{\Sigma}| & =\left|\boldsymbol{\Sigma}_{11}\right|\left|\boldsymbol{\Sigma}_{22}-\boldsymbol{\Sigma}_{21} \boldsymbol{\Sigma}_{11}^{-1} \boldsymbol{\Sigma}_{12}\right| \\
& =\left|\boldsymbol{\Sigma}_{22}\right|\left|\boldsymbol{\Sigma}_{11}-\boldsymbol{\Sigma}_{12} \boldsymbol{\Sigma}_{22}^{-1} \boldsymbol{\Sigma}_{21}\right| .
\end{aligned}
$$

Isto ocorre, sempre que as matrizes $\boldsymbol{\Sigma}_{11}$ e $\boldsymbol{\Sigma}_{22}$ forem quadradas e não singulares.

Então, podemos escrever a equação (C.3) como,

$$
\begin{aligned}
f_{\mathbf{Y}_{2}}\left(\mathbf{y}_{2}\right) & =\left|\boldsymbol{\Sigma}_{22}\right|^{-1 / 2} \int_{R^{n}}\left|\boldsymbol{\Sigma}_{11}-\boldsymbol{\Sigma}_{12} \boldsymbol{\Sigma}_{22}^{-1} \boldsymbol{\Sigma}_{21}\right|^{-1 / 2} g\left\{(\mathbf{Y}-\boldsymbol{\mu})^{\top} \boldsymbol{\Sigma}^{-1}(\mathbf{Y}-\boldsymbol{\mu})\right\} d \mathbf{y}_{1} \\
& =\left|\boldsymbol{\Sigma}_{22}\right|^{-1 / 2} g_{2}\left\{\left(\mathbf{Y}_{2}-\boldsymbol{\mu}_{2}\right)^{\top} \boldsymbol{\Sigma}_{22}^{-1}\left(\mathbf{Y}_{2}-\boldsymbol{\mu}_{2}\right)\right\} .
\end{aligned}
$$

Assim, a função $g_{2}(\cdot)$ dependerá da resolução da integral.

3. Usando a partição do item anterior, com $0<n<m$, temos que

$$
\left(\mathbf{Y}_{1} \mid \mathbf{Y}_{2}=\mathbf{y}_{2}\right) \stackrel{d}{=} \boldsymbol{\mu}_{1.2}+r_{q\left(\mathbf{y}_{2}\right)} \mathbf{A}_{11.2}^{\top} u^{(n)} \sim E l_{n}\left(\boldsymbol{\mu}_{1.2}, \boldsymbol{\Sigma}_{11.2}, \phi_{q\left(\mathbf{y}_{2}\right)}\right),
$$

em que

$$
\begin{aligned}
\boldsymbol{\mu}_{1.2} & =\boldsymbol{\mu}_{1}+\boldsymbol{\Sigma}_{12} \boldsymbol{\Sigma}_{22}^{-1}\left(y_{2}-\boldsymbol{\mu}_{2}\right) \\
\boldsymbol{\Sigma}_{11.2} & =\boldsymbol{\Sigma}_{11}-\boldsymbol{\Sigma}_{12} \boldsymbol{\Sigma}_{22}^{-1} \boldsymbol{\Sigma}_{21}=\mathbf{A}_{11.2}^{\top} \mathbf{A}_{11.2}
\end{aligned}
$$


$\mathrm{e}$

$$
q\left(\mathbf{y}_{2}\right)=\left(\mathbf{y}_{2}-\boldsymbol{\mu}_{2}\right)^{\top} \boldsymbol{\Sigma}_{22}^{-1}\left(\mathbf{y}_{2}-\boldsymbol{\mu}_{2}\right) .
$$

Agora, se existe a função de densidade de $\mathbf{Y}$ usaremos a seguinte notação:

$$
\left(\mathbf{Y}_{1} \mid \mathbf{Y}_{2}=\mathbf{y}_{2}\right) \stackrel{d}{=} \boldsymbol{\mu}_{1.2}+r_{q\left(\mathbf{y}_{2}\right)} \mathbf{A}_{11.2}^{\top} u^{(n)} \sim E l_{n}\left(\boldsymbol{\mu}_{1.2}, \boldsymbol{\Sigma}_{11.2}, g_{q\left(\mathbf{y}_{2}\right)}\right)
$$

em que $g_{q\left(\mathbf{y}_{2}\right)}$ é a função geradora associada à função de densidade da distribuição condicional $\mathbf{Y}_{1} \mid \mathbf{Y}_{2}$ e pode ser obtida desde,

$$
\begin{aligned}
f\left(\mathbf{Y}_{1} \mid \mathbf{Y}_{2}\right) & =\frac{f_{\mathbf{Y}}(\mathbf{y})}{f_{\mathbf{Y}_{2}\left(\mathbf{y}_{2}\right)}}=\frac{|\mathbf{\Sigma}|^{-1 / 2} g\left\{(\mathbf{Y}-\boldsymbol{\mu})^{\top} \boldsymbol{\Sigma}^{-1}(\mathbf{Y}-\boldsymbol{\mu})\right\}}{\left|\Sigma_{22}\right|^{-1} g_{2}\left(q\left(\mathbf{y}_{2}\right)\right)} \\
& =\frac{\left|\Sigma_{11.2}\right|^{-1 / 2}\left|\Sigma_{22}\right|^{-1 / 2} g\left\{\left(\mathbf{Y}_{1}-\boldsymbol{\mu}_{1.2}\right)^{\top} \boldsymbol{\Sigma}_{11.2}^{-1}\left(\mathbf{Y}_{1}-\boldsymbol{\mu}_{1.2}\right)+q\left(\mathbf{y}_{2}\right)\right\}}{\left|\boldsymbol{\Sigma}_{22}\right|^{-1 / 2} g_{2}\left(q\left(\mathbf{y}_{2}\right)\right)} \\
& =\left|\Sigma_{11.2}\right|^{-1 / 2} \frac{g\left\{\left(\mathbf{Y}_{1}-\boldsymbol{\mu}_{1.2}\right)^{\top} \boldsymbol{\Sigma}_{11.2}^{-1}\left(\mathbf{Y}_{1}-\boldsymbol{\mu}_{1.2}\right)+q\left(\mathbf{y}_{2}\right)\right\}}{g_{2}\left(q\left(\mathbf{y}_{2}\right)\right)} \\
& =\left|\Sigma_{11.2}\right|^{-1 / 2} g_{q\left(\mathbf{y}_{2}\right)}\left\{\left(\mathbf{Y}_{1}-\boldsymbol{\mu}_{1.2}\right)^{\top} \boldsymbol{\Sigma}_{11.2}^{-1}\left(\mathbf{Y}_{1}-\boldsymbol{\mu}_{1.2}\right)\right\}
\end{aligned}
$$

em que $g_{2}(\cdot)$ é a função geradora que define a densidade marginal de $\mathbf{Y}_{2}$ e portanto, a última igualdade mostra que a distribuição condicional também tem distribuição elíptica com função geradora de densidade $g_{q\left(\mathbf{y}_{2}\right)}$ é dada pela razão de $g(\mathbf{y})$ e $g_{2}\left(q\left(\mathbf{y}_{2}\right)\right)$. Para ilustrar os cálculos, mostraremos um caso particular, quando $\mathbf{Y}$ tem distribuição t-Student multivariada com parâmetros, $\boldsymbol{\mu}$ e $\boldsymbol{\Sigma}$. Temos que a distribuição condicional de $\mathbf{Y}_{1} \mid \mathbf{Y}_{2}$, é dada por

$$
\mathbf{Y}_{1} \mid \mathbf{Y}_{2} \sim t_{n}\left(\boldsymbol{\mu}_{1.2}, \mathbf{\Sigma}_{11.2}, g_{q\left(\mathbf{y}_{2}\right)}, \nu\right)
$$

Da equação (C.4) temos que $g_{q\left(\mathbf{y}_{2}\right)}$ é a razão de $g(\mathbf{y})$ e $g_{2}\left(q\left(\mathbf{y}_{2}\right)\right)$ e da Tabela 3.1 temos que $g(u)=c_{2}(\nu+u)^{-(\nu+m) / 2}, \operatorname{com} u=(\mathbf{Y}-\boldsymbol{\mu})^{\top} \boldsymbol{\Sigma}^{-1}(\mathbf{Y}-\boldsymbol{\mu})$ e $q\left(\mathbf{y}_{2}\right)=u_{2}$. Portanto, a densidade condicional fica dada por

$$
\begin{aligned}
f\left(\mathbf{V}_{1} \mid \mathbf{V}_{2}\right) & =\left|\boldsymbol{\Sigma}_{11.2}\right|^{-1 / 2} \frac{c_{2}\left(\nu+(\mathbf{Y}-\boldsymbol{\mu})^{\top} \boldsymbol{\Sigma}^{-1}(\mathbf{Y}-\boldsymbol{\mu})\right)^{-(\nu+m) / 2}}{c_{q\left(\mathbf{y}_{2}\right)}\left(\nu+\left(\mathbf{y}_{2}-\boldsymbol{\mu}_{2}\right)^{\top} \boldsymbol{\Sigma}_{22}^{-1}\left(\mathbf{y}_{2}-\boldsymbol{\mu}_{2}\right)\right)^{-(\nu+m-n) / 2}} \\
& =\left|\boldsymbol{\Sigma}_{11.2}\right|^{-1 / 2} \frac{c_{2}}{c_{q\left(\mathbf{y}_{2}\right)}} \lambda_{q\left(\mathbf{y}_{2}\right)}^{-(\nu+m-n) / 2}\left(\lambda_{q\left(\mathbf{y}_{2}\right)}+\left(\mathbf{Y}_{1}-\boldsymbol{\mu}_{1.2}\right)^{\top} \boldsymbol{\Sigma}_{11.2}^{-1}\left(\mathbf{Y}_{1}-\boldsymbol{\mu}_{1.2}\right)\right)^{-(\nu+m) / 2}
\end{aligned}
$$

$\operatorname{com} \lambda_{q\left(\mathbf{y}_{2}\right)}=\nu+q\left(y_{2}\right)$ e da equação (3.2) as funções $c_{2}$ e $c_{q\left(y_{2}\right)}$ são,

$$
c_{2}=k(m, \nu) \nu^{\nu / 2} \text { e } \quad c_{q\left(\mathbf{y}_{2}\right)}=k(m-n, \nu) \nu^{\nu / 2},
$$

em que $k(m, \nu)$ é uma função que depende da dimensão da distribuição e dos graus de liberdades $\nu$. Então, temos que

$$
\begin{aligned}
\frac{c_{2}}{c_{2 u_{2}}} & =\frac{k(m, \nu) \nu^{\nu / 2}}{k(m-n, \nu) \nu^{\nu / 2}}=\frac{\frac{\Gamma\{1 / 2(\nu+m)\}}{\pi^{m / 2} \Gamma(\nu / 2)}}{\frac{\Gamma\{1 / 2(\nu+m-n)\}}{\pi^{(m-n) / 2} \Gamma(\nu / 2)}} \\
& =\frac{\Gamma\{1 / 2(\nu+m)\}}{\pi^{n / 2} \Gamma\{1 / 2(\nu+m-n)\}}=k(n, v+m-n) .
\end{aligned}
$$


Assim, a função de densidade da distribuição condicional $\mathbf{Y}_{1} \mid \mathbf{Y}_{2}$, fica dada por

$$
\begin{aligned}
f\left(\mathbf{V}_{1} \mid \mathbf{V}_{2}\right)= & \left|\boldsymbol{\Sigma}_{11.2}\right|^{-1 / 2} k(n, v+m-n) \lambda_{q\left(y_{2}\right)}^{-(\nu+m-n) / 2} \\
& \times\left(\lambda_{q\left(y_{2}\right)}+\left(\mathbf{Y}_{1}-\boldsymbol{\mu}_{1.2}\right)^{\top} \boldsymbol{\Sigma}_{11.2}^{-1}\left(\mathbf{Y}_{1}-\boldsymbol{\mu}_{1.2}\right)\right)^{-(\nu+m) / 2} \\
= & \left|\boldsymbol{\Sigma}_{11.2}\right|^{-1 / 2} g_{q\left(y_{2}\right)}\left(\left(\mathbf{Y}_{1}-\boldsymbol{\mu}_{1.2}\right)^{\top} \boldsymbol{\Sigma}_{11.2}^{-1}\left(\mathbf{Y}_{1}-\boldsymbol{\mu}_{1.2}\right)\right) .
\end{aligned}
$$

A função de densidade resultante corresponde a uma distribuição t-Student generalizada que denotaremos como

$$
\mathbf{Y}_{1} \mid \mathbf{Y}_{2} \sim t_{n}\left(\boldsymbol{\mu}_{1.2}, \boldsymbol{\Sigma}_{11.2}, \nu+m-n, \lambda_{q\left(y_{2}\right)}\right)
$$

em que $\lambda_{q\left(y_{2}\right)}$ não contém novos parâmetros desconhecidos, sendo uma função dos graus de liberdade, vetor de médias e matriz de escala do vetor aleatório $Y_{2}$.

4. A forma quadrática $u=(\mathbf{Y}-\boldsymbol{\mu})^{\top} \boldsymbol{\Sigma}^{-1}(\mathbf{Y}-\boldsymbol{\mu})$ tem a mesma distribuição que $r^{2}$, isto pode ser denotado como

$$
(\mathbf{Y}-\boldsymbol{\mu})^{\top} \boldsymbol{\Sigma}^{-1}(\mathbf{Y}-\boldsymbol{\mu}) \stackrel{d}{=} r^{2}
$$

em que $r$ é a variável aleatória descrita na representação estocástica de uma distribuição elíptica, dada na equação (C.1). Assim, quando o vetor $\mathbf{Y}$, de dimensão $m$, tem distribuição normal, $r^{2}$ tem distribuição qui-quadrado com $m$ graus de liberdade. Agora, se o vetor $\mathbf{Y}$ de dimensão $m$ tem distribuição t-Student com $\nu$ graus de liberdade, $r^{2} / m$ tem distribuição $\mathrm{F}$ com $m$ e $\nu$ graus de liberdade. 


\section{Apêndice D}

\section{Modelo linear misto t-Student}

\section{D.1 Equações de estimação}

Equações de estimação do $j$-ésimo elemento do vetor $\boldsymbol{\theta}$, com $j=1, \ldots, s$.

- Função de Verossimilhança

$$
\begin{aligned}
\frac{\partial}{\partial \theta_{j}} l(\boldsymbol{\beta}, \boldsymbol{\theta}) & =\frac{\partial}{\partial \theta_{j}}\left[-\frac{1}{2} \sum_{i=1}^{n} \log \left|\boldsymbol{\Sigma}_{i}\right|+\sum_{i=1}^{n} \frac{\nu+m_{i}}{2} \log \left(1+u_{i} / \nu\right)\right] \\
& =-\frac{1}{2} \sum_{i=1}^{n} \operatorname{tr}\left\{\boldsymbol{\Sigma}_{i}^{-1} \frac{\partial \boldsymbol{\Sigma}_{i}}{\partial \theta_{j}}\right\}-\sum_{i=1}^{n}\left\{\left(\nu+m_{i}\right) / 2 \nu\right\}\left(1+u_{i} / \nu\right)^{-1}\left[\frac{\partial u_{i}}{\partial \theta_{j}}\right]=0 .
\end{aligned}
$$

- Verossimilhança restrita

$$
\begin{aligned}
-\frac{1}{2} \sum_{i=1}^{n} \operatorname{tr}\left\{\boldsymbol{\Sigma}_{i}^{-1} \frac{\partial \boldsymbol{\Sigma}_{i}}{\partial \theta_{j}}\right\} & -\frac{1}{2} \sum_{i=1}^{n} \operatorname{tr}\left\{\left(\mathbf{X}_{i}^{\top} \boldsymbol{\Sigma}_{i}^{-1} \mathbf{X}_{i}\right)^{-1} \mathbf{X}_{i}^{\top} \boldsymbol{\Sigma}_{i}^{-1} \frac{\partial \boldsymbol{\Sigma}_{i}}{\partial \theta_{j}} \boldsymbol{\Sigma}_{i}^{-1} \mathbf{X}_{i}\right\} \\
& +\frac{1}{2 \nu} \sum_{i=1}^{n}\left(\nu+m_{i}-p\right)\left(1+u_{i 2} / \nu\right)^{-1}\left[\frac{\partial u_{i 2}}{\partial \theta_{j}}\right]=0,
\end{aligned}
$$

em que $u_{i 2}=\left(\mathbf{Y}_{i}-\mathbf{X}_{i} \mathbf{b}_{i}\right)^{\top} \boldsymbol{\Sigma}_{i}^{-1}\left(\mathbf{Y}_{i}-\mathbf{X}_{i} \mathbf{b}_{i}\right)$ e $\mathbf{b}_{i}=\left(\mathbf{X}_{i}^{\top} \boldsymbol{\Sigma}_{i}^{-1} \mathbf{X}_{i}\right)^{-1} \mathbf{X}_{i}^{\top} \boldsymbol{\Sigma}_{i}^{-1} \mathbf{Y}_{i}$.

- Verossimilhança perfilada modificada

$$
\begin{gathered}
\frac{1}{4} \operatorname{tr}\left[\left\{\sum_{i=1}^{n} \frac{\nu+m_{i}}{\nu}\left(1+\hat{u}_{i} / \nu\right)^{-2} \mathbf{X}_{i}^{\top} \boldsymbol{\Sigma}_{i}^{-1} \mathbf{X}_{i}\right\}^{-1} \sum_{i=1}^{n} \frac{\nu+m_{i}}{\nu^{3}}\left(1+\hat{u}_{i} / \nu\right)^{-2}\right. \\
\left.\left\{\left(1+\hat{u}_{i} / \nu\right)^{-1}\left[\frac{\partial \hat{u}_{i}}{\partial \theta_{j}}\right] \mathbf{X}_{i}^{\top} \boldsymbol{\Sigma}_{i}^{-1} \mathbf{X}_{i}+\mathbf{X}_{i}^{\top} \boldsymbol{\Sigma}_{i}^{-1} \frac{\partial \boldsymbol{\Sigma}_{i}}{\partial \theta_{j}} \boldsymbol{\Sigma}_{i}^{-1} \mathbf{X}_{i}\right\}\right] \\
-\frac{1}{2} \sum_{i=1}^{n} \operatorname{tr}\left\{\boldsymbol{\Sigma}_{i}^{-1} \frac{\partial \boldsymbol{\Sigma}_{i}}{\partial \theta_{j}}\right\}-\frac{1}{2} \sum_{i=1}^{n} \frac{\nu+m_{i}}{\nu}\left(1+\hat{u}_{i} / \nu\right)^{-1}\left[\frac{\partial \hat{u}_{i}}{\partial \theta_{j}}\right]=0
\end{gathered}
$$

em que $\hat{u}_{i}=\left(\mathbf{Y}_{i}-\mathbf{X}_{i} \hat{\boldsymbol{\beta}}_{\theta}\right)^{\top} \boldsymbol{\Sigma}_{i}^{-1}\left(\mathbf{Y}_{i}-\mathbf{X}_{i} \hat{\boldsymbol{\beta}}_{\theta}\right)$

- Verossimilhança perfilada modificada aproximada

$$
\frac{1}{4} \operatorname{tr}\left[\left\{\sum_{i=1}^{n} \frac{\nu^{2}\left(\nu+m_{i}\right)}{\nu+m_{i}+2} \mathbf{X}_{i}^{\top} \boldsymbol{\Sigma}_{i}^{-1} \mathbf{X}_{i}\right\}^{-1} \sum_{i=1}^{n} \frac{\nu^{2}\left(\nu+m_{i}\right)}{\nu+m_{i}+2} \mathbf{X}_{i}^{\top} \boldsymbol{\Sigma}_{i}^{-1} \frac{\partial \boldsymbol{\Sigma}_{i}}{\partial \theta_{j}} \boldsymbol{\Sigma}_{i}^{-1} \mathbf{X}_{i}\right]
$$




$$
-\frac{1}{2} \sum_{i=1}^{n} \operatorname{tr}\left\{\boldsymbol{\Sigma}_{i}^{-1} \frac{\partial \boldsymbol{\Sigma}_{i}}{\partial \theta_{j}}\right\}-\frac{1}{2} \sum_{i=1}^{n}\left(\nu+m_{i}\right)\left(\nu+\hat{u}_{i}\right)^{-1}\left[\frac{\partial \hat{u}_{i}}{\partial \theta_{j}}\right]=0 .
$$

\section{D.2 Cálculos e derivadas}

\section{D.2.1 Matriz de informação}

As respectivas matrizes de informação são dadas por

$$
\begin{gathered}
\frac{\partial^{2}}{\partial \boldsymbol{\beta}^{\top} \partial \boldsymbol{\beta}} l(\boldsymbol{\beta}, \boldsymbol{\theta})=4 \sum_{i=1}^{n}\left\{\left(\nu+m_{i}\right) / 2 \nu^{2}\right\}\left(1+u_{i} / \nu\right)^{-2} \mathbf{X}_{i}^{\top} \boldsymbol{\Sigma}_{i}^{-1} \mathbf{X}_{i}, \\
\frac{\partial^{2}}{\partial \theta_{j} \partial \boldsymbol{\beta}} l(\boldsymbol{\beta}, \boldsymbol{\theta})=2 \sum_{i=1}^{n}\left\{\left(\nu+m_{i}\right) / 2 \nu^{2}\right\}\left(1+u_{i} / \nu\right)^{-2}\left[\frac{\partial u_{i}}{\partial \theta_{j}}\right] \mathbf{X}_{i}^{\top} \boldsymbol{\Sigma}_{i}^{-1}\left(\mathbf{Y}_{i}-\mathbf{X}_{i} \boldsymbol{\beta}\right) \\
+2 \sum_{i=1}^{n}\left\{\left(\nu+m_{i}\right) / 2 \nu\right\}\left(1+u_{i} / \nu\right)^{-1} \mathbf{X}_{i}^{\top} \boldsymbol{\Sigma}_{i}^{-1} \frac{\partial \boldsymbol{\Sigma}_{i}}{\partial \theta_{j}} \boldsymbol{\Sigma}_{i}^{-1}\left(\mathbf{Y}_{i}-\mathbf{X}_{i} \boldsymbol{\beta}\right)
\end{gathered}
$$

e

$$
\begin{aligned}
\frac{\partial^{2}}{\partial \theta_{k} \partial \theta_{j}} l(\boldsymbol{\beta}, \boldsymbol{\theta})= & \frac{1}{2} \sum_{i=1}^{n} \operatorname{tr}\left\{\boldsymbol{\Sigma}_{i}^{-1} \frac{\partial \boldsymbol{\Sigma}_{i}}{\partial \theta_{k}} \boldsymbol{\Sigma}_{i}^{-1} \frac{\partial \boldsymbol{\Sigma}_{i}}{\partial \theta_{j}}\right\}+\sum_{i=1}^{n}\left\{\left(\nu+m_{i}\right) / 2 \nu^{2}\right\}\left(1+u_{i} / \nu\right)^{-2}\left[\frac{\partial u_{i}}{\partial \theta_{k}}\right]\left[\frac{\partial u_{i}}{\partial \theta_{j}}\right] \\
& +\sum_{i=1}^{n}\left\{\left(\nu+m_{i}\right) / 2 \nu\right\}\left(1+u_{i} / \nu\right)^{-1}\left[\frac{\partial^{2} u_{i}}{\partial \theta_{k} \partial \theta_{j}}\right]
\end{aligned}
$$

em que a derivada parcial de $u_{i}$ com respeito ao $j$-ésimo elemento do vetor $\boldsymbol{\theta}$ fica dada por

$$
\frac{\partial u_{i}}{\partial \theta_{j}}=\left(\mathbf{Y}_{i}-\mathbf{X}_{i} \boldsymbol{\beta}\right)^{\top} \boldsymbol{\Sigma}_{i}^{-1} \frac{\partial \boldsymbol{\Sigma}_{i}}{\partial \theta_{j}} \boldsymbol{\Sigma}_{i}^{-1}\left(\mathbf{Y}_{i}-\mathbf{X}_{i} \boldsymbol{\beta}\right)
$$

para qualquer $k$ distinto de $j$ com $k, j=1, \ldots, s$ temos que

$$
\frac{\partial u_{i}}{\partial \theta_{k}} \frac{\partial u_{i}}{\partial \theta_{j}}=\left(\mathbf{Y}_{i}-\mathbf{X}_{i} \boldsymbol{\beta}\right)^{\top} \boldsymbol{\Sigma}_{i}^{-1} \frac{\partial \boldsymbol{\Sigma}_{i}}{\partial \theta_{k}} \boldsymbol{\Sigma}_{i}^{-1}\left(\mathbf{Y}_{i}-\mathbf{X}_{i} \boldsymbol{\beta}\right)\left(\mathbf{Y}_{i}-\mathbf{X}_{i} \boldsymbol{\beta}\right)^{\top} \boldsymbol{\Sigma}_{i}^{-1} \frac{\partial \boldsymbol{\Sigma}_{i}}{\partial \theta_{j}} \boldsymbol{\Sigma}_{i}^{-1}\left(\mathbf{Y}_{i}-\mathbf{X}_{i} \boldsymbol{\beta}\right)
$$

e

$$
\frac{\partial^{2} u_{i}}{\partial \theta_{k} \partial \theta_{j}}=\left(\mathbf{Y}_{i}-\mathbf{X}_{i} \boldsymbol{\beta}\right)^{\top}\left\{2 \boldsymbol{\Sigma}_{i}^{-1} \frac{\partial \boldsymbol{\Sigma}_{i}}{\partial \theta_{k}} \boldsymbol{\Sigma}_{i}^{-1} \frac{\partial \boldsymbol{\Sigma}_{i}}{\partial \theta_{j}} \boldsymbol{\Sigma}_{i}^{-1}+\boldsymbol{\Sigma}_{i}^{-1} \frac{\partial^{2} \boldsymbol{\Sigma}_{i}}{\partial \theta_{k} \theta_{j}} \boldsymbol{\Sigma}_{i}^{-1}\right\}\left(\mathbf{Y}_{i}-\mathbf{X}_{i} \boldsymbol{\beta}\right)
$$

\section{D.2.2 Matriz de informação esperada para $\beta$}

Baseados nos cálculos apresentados por Lange et al. (1989) para um modelo elíptico, consideramos as derivadas com respeito a dois elementos de $\boldsymbol{\beta}$, a variável $\mathbf{z}_{i}$, como $\mathbf{z}_{i}=\boldsymbol{\Sigma}_{i}^{-1 / 2}\left(\mathbf{Y}_{i}-\mathbf{X}_{i} \boldsymbol{\beta}\right)$ e $\mathbf{X}_{i l}^{\top}$ como sendo a $l$-ésima linha da matriz $\mathbf{X}_{i}, \operatorname{com} l=1, \cdots, m$ e seja $l^{\prime}$ outra linha da matriz $\mathbf{X}_{i}$ distinta a $l$. Então, obtemos

$$
\frac{\partial l_{i}(\boldsymbol{\beta}, \boldsymbol{\theta})}{\partial \boldsymbol{\beta}_{l}} \frac{\partial l_{i}(\boldsymbol{\beta}, \boldsymbol{\theta})}{\partial \boldsymbol{\beta}_{l^{\prime}}}=4\left\{\left(\nu+m_{i}\right) / 2\right\}^{2}\left(\nu+\left\|\mathbf{z}_{i}\right\|^{2}\right)^{-2} \mathbf{z}_{i}^{\top} \boldsymbol{\Sigma}_{i}^{-1 / 2} \mathbf{X}_{i l} \mathbf{X}_{i l^{\prime}}^{\top} \boldsymbol{\Sigma}_{i}^{-1 / 2} \mathbf{z}_{i} .
$$

Para encontrarmos a matriz de informação esperada, consideramos $\left\|\mathbf{z}_{i}\right\|^{2}=\mathbf{z}_{i}^{\top} \mathbf{z}_{i}$ $=\left(\mathbf{Y}_{i}-\mathbf{X}_{i} \boldsymbol{\beta}_{i}\right)^{\top} \boldsymbol{\Sigma}_{i}^{-1 / 2} \boldsymbol{\Sigma}_{i}^{-1 / 2}\left(\mathbf{Y}_{i}-\mathbf{X}_{i} \boldsymbol{\beta}\right)$. Assim, conforme mostrado por Lange et al. (1989) segue 
que

$$
\mathrm{E}\left\{\frac{\partial l_{i}(\boldsymbol{\beta}, \boldsymbol{\theta})}{\partial \boldsymbol{\beta}_{l}} \frac{\partial l_{i}(\boldsymbol{\beta}, \boldsymbol{\theta})}{\partial \boldsymbol{\beta}_{l^{\prime}}}\right\}=\frac{\nu^{2}\left(\nu+m_{i}\right)}{\nu+m_{i}+2} \mathbf{X}_{i l^{\prime}}^{\top} \boldsymbol{\Sigma}_{i}^{-1} \mathbf{X}_{i l}
$$

D.2.3 Derivadas parciais de $u_{i 2}$ e $\mathbf{b}_{i} \operatorname{com}$ respeito a $\theta_{j}$

$$
\begin{aligned}
\frac{\partial u_{i 2}}{\partial \theta_{j}} & =-2\left(\mathbf{Y}_{i}-\mathbf{X}_{i} \mathbf{b}_{i}\right)^{\top} \boldsymbol{\Sigma}_{i}^{-1} \mathbf{X}_{i}\left[\frac{\partial \mathbf{b}_{i}}{\partial \theta_{j}}\right]+\left(\mathbf{Y}_{i}-\mathbf{X}_{i} \mathbf{b}_{i}\right)^{\top} \boldsymbol{\Sigma}_{i}^{-1} \frac{\partial \boldsymbol{\Sigma}_{i}}{\partial \theta_{j}} \boldsymbol{\Sigma}_{i}^{-1}\left(\mathbf{Y}_{i}-\mathbf{X}_{i} \mathbf{b}_{i}\right) \\
& =\left(\mathbf{Y}_{i}-\mathbf{X}_{i} \mathbf{b}_{i}\right)^{\top}\left\{2 \boldsymbol{\Sigma}_{i}^{-1} \mathbf{X}_{i}\left(\mathbf{X}_{i}^{\top} \boldsymbol{\Sigma}_{i}^{-1} \mathbf{X}_{i}\right)^{-1} \mathbf{X}_{i}^{\top}+\mathbf{I}_{m_{i}}\right\} \boldsymbol{\Sigma}_{i}^{-1} \frac{\partial \boldsymbol{\Sigma}_{i}}{\partial \theta_{j}} \boldsymbol{\Sigma}_{i}^{-1}\left(\mathbf{Y}_{i}-\mathbf{X}_{i} \mathbf{b}_{i}\right)
\end{aligned}
$$

e

$$
\frac{\partial \mathbf{b}_{i}}{\partial \theta_{j}}=\left(\mathbf{X}_{i}^{\top} \boldsymbol{\Sigma}_{i}^{-1} \mathbf{X}_{i}\right)^{-1} \mathbf{X}_{i}^{\top} \boldsymbol{\Sigma}_{i}^{-1} \frac{\partial \boldsymbol{\Sigma}_{i}}{\partial \theta_{j}} \boldsymbol{\Sigma}_{i}^{-1}\left(\mathbf{Y}_{i}-\mathbf{X}_{i} \mathbf{b}_{i}\right)
$$

D.2.4 Derivadas parciais de $\hat{u}_{i}$ e $\hat{\boldsymbol{\beta}}_{\theta}$ com respeito a $\theta_{j}$

$$
\frac{\partial \hat{u}_{i}}{\partial \theta_{j}}=-2\left(\mathbf{Y}_{i}-\mathbf{X}_{i} \hat{\boldsymbol{\beta}}_{\theta}\right)^{\top} \boldsymbol{\Sigma}_{i}^{-1}\left[\frac{\partial \hat{\boldsymbol{\beta}}_{\theta}}{\partial \theta_{j}}\right]+\left(\mathbf{Y}_{i}-\mathbf{X}_{i} \hat{\boldsymbol{\beta}}_{\theta}\right)^{\top} \boldsymbol{\Sigma}_{i}^{-1} \frac{\partial \boldsymbol{\Sigma}_{i}}{\partial \theta_{j}} \boldsymbol{\Sigma}_{i}^{-1}\left(\mathbf{Y}_{i}-\mathbf{X}_{i} \hat{\boldsymbol{\beta}}_{\theta}\right)
$$

e

$$
\begin{aligned}
\frac{\partial \hat{\boldsymbol{\beta}}_{\theta}}{\partial \theta_{j}}= & {\left[-\sum_{i=1}^{n}\left\{\left(\nu+m_{i}\right) / 2 \nu^{2}\right\}\left(1+u_{i} / \nu\right)^{-2}\left[\frac{\partial u_{i}}{\partial \theta_{j}}\right] \mathbf{X}_{i}^{\top} \boldsymbol{\Sigma}_{i}^{-1} \mathbf{Y}_{i}+\sum_{i=1}^{n}\left\{\left(\nu+m_{i}\right) / 2 \nu\right\}\left(1+u_{i} / \nu\right)^{-1} \mathbf{X}_{i}^{\top} \boldsymbol{\Sigma}_{i}^{-1} \frac{\partial \boldsymbol{\Sigma}_{i}}{\partial \theta_{j}} \boldsymbol{\Sigma}_{i}^{-1} \mathbf{Y}_{i}\right] } \\
& \times\left[\sum_{i=1}^{n}\left\{\left(\nu+m_{i}\right) / 2 \nu\right\}\left(1+u_{i} / \nu\right)^{-1} \mathbf{X}_{i}^{\top} \boldsymbol{\Sigma}_{i}^{-1} \mathbf{X}_{i}\right] \\
& -\left[\sum_{i=1}^{n}\left\{\left(\nu+m_{i}\right) / 2 \nu\right\}\left(1+u_{i} / \nu\right)^{-1} \mathbf{X}_{i}^{\top} \boldsymbol{\Sigma}_{i}^{-1} \mathbf{Y}_{i}\right]\left[\sum_{i=1}^{n}\left\{\left(\nu+m_{i}\right) / 2 \nu\right\}\left(1+u_{i} / \nu\right)^{-1} \mathbf{X}_{i}^{\top} \boldsymbol{\Sigma}_{i}^{-1} \mathbf{X}_{i}\right]^{-2} \\
& \times\left[-\sum_{i=1}^{n}\left\{\left(\nu+m_{i}\right) / 2 \nu^{2}\right\}\left(1+u_{i} / \nu\right)^{-2}\left[\frac{\partial u_{i}}{\partial \theta_{j}}\right] \mathbf{X}_{i}^{\top} \boldsymbol{\Sigma}_{i}^{-1} \mathbf{X}_{i}\right. \\
& \left.+\sum_{i=1}^{n}\left\{\left(\nu+m_{i}\right) / 2 \nu\right\}\left(1+u_{i} / \nu\right)^{-1} \mathbf{X}_{i}^{\top} \boldsymbol{\Sigma}_{i}^{-1} \frac{\partial \boldsymbol{\Sigma}_{i}}{\partial \theta_{j}} \boldsymbol{\Sigma}_{i}^{-1} \mathbf{X}_{i}\right]
\end{aligned}
$$

para $j=1, \ldots, s$.

\section{D.3 Modelos simulados}

\section{D.3.1 Estrutura autoregressiva}

Considerando os resultados da Seção 3.5.4 para um modelo autoregressivo elíptico, em que $\boldsymbol{\Sigma}=\sigma^{2} \mathbf{R}$ e a matriz $\mathbf{R}$ foi especificado na equação (3.27), então temos que o vetor $\boldsymbol{\alpha}$ associado à matriz $\boldsymbol{\Sigma}$ é dado por $\boldsymbol{\alpha}=\left(\sigma^{2}, \rho\right)$. Para o estudo de simulação, diferente do apresentado na Seção 3.5.4 em que $\mathbf{X}$ é um vetor $(m \times 1)$, consideraremos a matriz $\mathbf{X}$ de dimensão $(m \times 2)$, isto é, que o vetor $\boldsymbol{\beta}$ contém dois parâmetros, dados por $\left(\beta_{1}, \beta_{2}\right)$. Como não temos expressões explícitas para os estimadores descreveremos brevemente o processo iterativo. Dado um valor inicial 
$\boldsymbol{\alpha}^{(0)}=\left(\sigma^{2^{(0)}}, \rho^{(0)}\right)$ obtemos um valor inicial para $\boldsymbol{\beta}^{(0)}$ dado por

$$
\boldsymbol{\beta}^{(0)}=\left(\mathbf{X}^{\top} \mathbf{R}^{-1(0)} \mathbf{X}\right)^{-1} \mathbf{X}^{\top} \mathbf{R}^{-1(0)} \mathbf{Y}
$$

Seja $r$ a $r$-ésima iteração do processo de estimação, com $r=0, \ldots$, Então, temos que $\boldsymbol{\alpha}^{(r)}=$ $\left(\sigma^{2(r)}, \rho^{(r)}\right)$ é a $r$-ésima estimativa para $\boldsymbol{\alpha}$ e que a $r$-ésima estimativa de $\boldsymbol{\beta}$ tem a mesma forma que $\boldsymbol{\beta}^{(0)}$, resultando em

$$
\boldsymbol{\beta}^{(r)}=\left(\mathbf{X}^{\top} \mathbf{R}^{(r)^{-1}} \mathbf{X}\right)^{-1} \mathbf{X}^{\top} \mathbf{R}^{(r)^{-1}} \mathbf{Y}
$$

Assim, podemos escrever um algoritmo de estimação para cada método.

\section{Método de máxima verossimilhança}

Denotaremos por $\boldsymbol{\alpha}_{M V}^{(r)}$ e $\boldsymbol{\beta}_{M V}^{(r)}$ as estimativas obtidas na $r$-ésima iteração do processo de estimação de máxima verossimilhança de $\boldsymbol{\alpha}$ e de $\boldsymbol{\beta}$, respectivamente. Então, na $(r+1)$ iteração temos:

Passo 1: Considerando $\boldsymbol{\alpha}_{M V}^{(r)}$ o valor inicial, a estimativa de máxima verossimilhança de $\boldsymbol{\alpha}$ na $(r+1)$ iteração pode ser obtida maximizando o logaritmo da função de verossimilhança dado por

$$
l(\boldsymbol{\beta}, \boldsymbol{\alpha} ; \mathbf{Y})=-\frac{m}{2} \log \sigma^{2}-\frac{1}{2} \log |\mathbf{R}|-\frac{\nu+m}{2} \log \left[1+\left\{\frac{1}{\sigma^{2} \nu}(\mathbf{Y}-\mathbf{X} \boldsymbol{\beta})^{\top} \mathbf{R}^{-1}(\mathbf{Y}-\mathbf{X} \boldsymbol{\beta})\right\}\right] .
$$

Passo 2: Considerando a estimativa $(r+1)$ de $\rho$, obtida no passo anterior, a atualização da estimativa de máxima verossimilhança do vetor $\boldsymbol{\beta}$ na iteração $(r+1)$ será dada por

$$
\boldsymbol{\beta}_{M V}^{(r+1)}=\left(\mathbf{X}^{\top} \mathbf{R}_{M V}^{(r)}{ }^{-1} \mathbf{X}\right)^{-1} \mathbf{X}^{\top} \mathbf{R}_{M V}^{(r)}{ }^{-1} \mathbf{Y} .
$$

\section{Método de máxima verossimilhança restrita}

Seja $\boldsymbol{\alpha}_{M V R}^{(r)}=\left(\sigma_{M V R}^{2}{ }^{(r)}, \rho_{M V R}^{(r)}\right)$ a estimativa de máxima verossimilhança restrita do vetor $\boldsymbol{\alpha}$ obtida na $r$-ésima iteração. Então, na iteração $(r+1)$ maximizamos o logaritmo da função de verossimilhança de $\mathbf{V}_{2}$ dado por

$$
\begin{array}{r}
l\left(\boldsymbol{\alpha} ; \mathbf{V}_{2}\right)=-\frac{m}{2} \log \sigma^{2}-\frac{1}{2} \log |\mathbf{R}|+\frac{p}{2} \log \sigma^{2}-\frac{1}{2} \log \left|\mathbf{X}^{\top} \mathbf{R}^{-1} \mathbf{X}\right| \\
-\frac{\nu+m-p}{2} \log \left[1+\left\{\frac{1}{\sigma^{2} \nu}\left(\mathbf{Y}-\mathbf{X B}_{\mathbf{R}}\right)^{\top} \mathbf{R}^{-1}\left(\mathbf{Y}-\mathbf{X B}_{\mathbf{R}}\right)\right\}\right]
\end{array}
$$

$\operatorname{com} \mathbf{B}_{\mathbf{R}}=\left(\mathbf{X}^{\top} \mathbf{R}^{-1} \mathbf{X}\right)^{-1} \mathbf{X}^{\top} \mathbf{R}^{-1} \mathbf{Y}$.

Uma vez obtida a estimativa restrita do vetor $\boldsymbol{\alpha}$, a estimativa restrita do vetor de $\boldsymbol{\beta}$ é dada por

$$
\hat{\boldsymbol{\beta}}_{M V R}=\left(\mathbf{X}^{\top} \widehat{\mathbf{R}}_{M V R}^{-1} \mathbf{X}\right)^{-1} \mathbf{X}^{\top} \widehat{\mathbf{R}}_{M V R}^{-1} \mathbf{Y}
$$

em que $\widehat{\mathbf{R}}_{M V R}$ denota a estimativa restrita da matriz $\mathbf{R}$ resultante no processo de maximização.

\section{Método de máxima verossimilhança perfilada modificada}

Sendo o vetor $\boldsymbol{\alpha}_{M V P M}^{(r)}$ como a estimativa de máxima verossimilhança perfilada modificada obtida na $r$-ésima iteração. Na $(r+1)$-iteração, considerando $\boldsymbol{\alpha}_{M V P M}^{(r)}$ como o valor inicial, temos que o vetor $\boldsymbol{\alpha}_{M V P M}^{(r+1)}$ é obtido da maximização a função de verossimilhança perfilada modificada, 
dada por

$l_{p m}^{*}(\boldsymbol{\alpha})=l_{p}(\boldsymbol{\alpha})+\frac{p}{2} \log \sigma^{2}+\frac{p}{2} \log \left|1+\left\{\frac{1}{\sigma^{2} \nu}\left(\mathbf{Y}-\mathbf{X B}_{\mathbf{R}}\right)^{\top} \mathbf{R}^{-1}\left(\mathbf{Y}-\mathbf{X B}_{\mathbf{R}}\right)\right\}\right|-\frac{1}{2} \log \left|\mathbf{X}^{\top} \mathbf{R}^{-1} \mathbf{X}\right|$,

em que $l_{p}(\boldsymbol{\alpha})$ a função perfilada do vetor $\boldsymbol{\alpha}$, dada por

$$
l_{p}(\boldsymbol{\alpha})=-\frac{m}{2} \log \sigma^{2}-\frac{1}{2} \log |\mathbf{R}|+\log g\left\{\frac{1}{\sigma^{2}}\left(\mathbf{Y}-\mathbf{X B}_{\mathbf{R}}\right)^{\top} \mathbf{R}^{-1}\left(\mathbf{Y}-\mathbf{X B}_{\mathbf{R}}\right)\right\} .
$$

A atualização da estimativa do vetor de $\boldsymbol{\beta}$ é dada por

$$
\hat{\boldsymbol{\beta}}_{M V P M}=\left(\mathbf{X}^{\top} \widehat{\mathbf{R}}_{M V P M}^{-1} \mathbf{X}\right)^{-1} \mathbf{X}^{\top} \widehat{\mathbf{R}}_{M V P M}^{-1} \mathbf{Y},
$$

em que $\widehat{\mathbf{R}}_{M V P M}$ denota a estimativa de máxima verossimilhança perfilada modificada da matriz R.

\section{Método de máxima verossimilhança perfilada modificada aproximada}

Similar ao caso anterior temos que a função de verossimilhança perfilada modificada aproximada é dada por

$$
l_{p m a}^{*}(\boldsymbol{\alpha})=l_{p}(\boldsymbol{\alpha})-\frac{p}{2} \log \sigma^{2}-\frac{p}{2} \log \left|\frac{\nu+m}{2 \pi(\nu+m+2)}\right|-\frac{1}{2} \log \left|\mathbf{X}^{\top} \mathbf{R}^{-1} \mathbf{X}\right|,
$$

em que $l_{p}(\boldsymbol{\alpha})$ foi especificada na equação (D.1). Então, considerando os vetores $\boldsymbol{\alpha}_{M V P M A}^{(r)}$ e $\boldsymbol{\beta}_{M V P M A}^{(r)}$ como as estimativas de máxima verossimilhança perfilada modificada aproximada obtidas na $r$-ésima iteração, temos que o processo na $(r+1)$ iteração fica dado por:

- Obter o vetor $\boldsymbol{\alpha}_{M V P M A}^{(r+1)}$ da maximização de função de verossimilhança perfilada modificada considerando o vetor $\boldsymbol{\alpha}_{M V P M A}^{(r)}$ como valor inicial.

- A atualização da estimativa do vetor de $\boldsymbol{\beta}$ na $(r+1)$ iteração é dada por

$$
\hat{\boldsymbol{\beta}}_{M V P M A}=\left(\mathbf{X}^{\top} \widehat{\mathbf{R}}_{M V P M A}^{-1} \mathbf{X}\right)^{-1} \mathbf{X}^{\top} \widehat{\mathbf{R}}_{M V P M A}^{-1} \mathbf{Y},
$$

em que $\widehat{\mathbf{R}}_{M V P M A}$ denota estimativa de máxima verossimilhança perfilada modificada aproximada da matriz $\mathbf{R}$.

\section{D.3.2 Modelo misto linear t-Student}

Considerando o modelo apresentado na equação (4.1) do Capítulo 4 e as especificações para o modelo misto linear t-Student dadas na Seção 4.3.2 temos que a matriz $\boldsymbol{\Sigma}_{i}$ é dada por $\mathbf{Z}_{i} \mathbf{D}_{i} \mathbf{Z}_{i}^{\top}+\boldsymbol{\Sigma}_{m_{i}}$ e $\boldsymbol{\theta}=\left(\boldsymbol{\gamma}^{\top}, \boldsymbol{\alpha}^{\top}\right)^{\top}$ é o vetor de parâmetros desconhecidos as matrizes $\boldsymbol{\Sigma}_{i}$, com $i=1, \ldots, n$. A continuação descrevemos, brevemente o processo de estimação para cada método de estimação. Consideramos, em geral, para $\boldsymbol{\theta}^{\top}=(\boldsymbol{\alpha}, \boldsymbol{\gamma})$ temos valores iniciais $\boldsymbol{\theta}^{(0)}=\left(\boldsymbol{\alpha}^{(0)}, \boldsymbol{\gamma}^{(0)}\right)$ e para $\boldsymbol{\beta}$ temos $\boldsymbol{\beta}^{(0)}$. Na seção (B.3) do Apêndice B apresentamos alguns resultados das estruturas da matriz $\boldsymbol{\Sigma}$ que podem ser utilizadas.

Então, denotaremos por $r$ a $r$-ésima iteração do processo de estimação, com $r=0, \ldots$ Assim, temos que $\boldsymbol{\theta}^{(r)}=\left(\boldsymbol{\alpha}^{(r)}, \boldsymbol{\gamma}^{(r)}\right)$ é a $r$-ésima estimativa para $\boldsymbol{\theta}$ e que a $(r+1)$-ésima estimativa de $\boldsymbol{\beta}$ resulta dada por

$$
\boldsymbol{\beta}_{M}^{(r+1)}=\left[\sum_{i=1}^{n} \frac{\nu+m_{i}}{\nu+u_{i M}^{(r)}} \mathbf{X}_{i}^{\top}\left\{\boldsymbol{\Sigma}_{i M}^{(r)}\right\}^{-1} \mathbf{X}_{i}\right]^{-1}\left[\sum_{i=1}^{n} \frac{\nu+m_{i}}{\nu+u_{i M}^{(r)}} \mathbf{X}_{i}^{\top}\left\{\boldsymbol{\Sigma}_{i M}^{(r)}\right\}^{-1} \mathbf{Y}_{i}\right]
$$


em que $u_{i}^{(r)}=\left(\mathbf{Y}_{i}-\mathbf{X}_{i} \boldsymbol{\beta}_{M}^{(r)}\right)^{\top} \boldsymbol{\Sigma}_{i M}^{(r)}{ }^{-1}\left(\mathbf{Y}_{i}-\mathbf{X}_{i} \boldsymbol{\beta}_{M}^{(r)}\right)$. Assim, podemos escrever um algoritmo de estimação para cada método.

\section{Método de máxima verossimilhança}

Denotaremos por $\boldsymbol{\theta}_{M V}^{(r)}$ e $\boldsymbol{\beta}_{M V}^{(r)}$ as estimativas obtidas na $r$-ésima iteração do processo de estimação de máxima verossimilhança de $\boldsymbol{\theta}$ e de $\boldsymbol{\beta}$, respectivamente. Então, a $(r+1)$-iteração pode ser obtida ao maximizar o logaritmo da função de verossimilhança com respeito a $\boldsymbol{\theta}$ e $\boldsymbol{\beta}$ em forma conjunta, considerando algum método de otimização e os valores iniciais são dados pelas estimativas obtidas na $r$-ésima maximização. Outra forma é separar o processo de estimação nos seguintes passos:

Passo1: Considerando $\boldsymbol{\theta}_{M V}^{(r)}$ e $\boldsymbol{\beta}_{M V}^{(r)}$ a estimativa de máxima verossimilhança de vetor $\boldsymbol{\theta}$ na $(r+1)$ iteração pode ser obtida maximizando o logaritmo da função de verossimilhança dada por

$l\left(\boldsymbol{\beta}_{M V}^{(r)}, \boldsymbol{\theta} ; \mathbf{Y}\right)$ que denota o logaritmo da função de verossimilhança avaliada na estimativa de máxima verossimilhança de $\boldsymbol{\beta}$ obtida na $r$-ésima iteração. A expressão do logaritmo da função de verossimilhança foi dado na equação (4.3) e as funções escore para $\boldsymbol{\beta}$ e $\boldsymbol{\theta}$ são dadas nas equações (4.4) e (4.5) do Capítulo 4.

Passo 2: Substituindo $\boldsymbol{\theta}_{M V}^{(r+1)}$ e $\boldsymbol{\beta}_{M V}^{(r)}$ na equação (4.17) obtemos a estimativa de máxima verossimilhança de $\boldsymbol{\beta}$ na $(r+1)$-iteração, isto é dado por

$$
\boldsymbol{\beta}_{M V}^{(r+1)}=\left[\sum_{i=1}^{n} \frac{\nu+m_{i}}{\nu+u_{i M V}^{(r)}} \mathbf{X}_{i}^{\top}\left\{\boldsymbol{\Sigma}_{i M V}^{(r)}\right\}^{-1} \mathbf{X}_{i}\right]^{-1}\left[\sum_{i=1}^{n} \frac{\nu+m_{i}}{\nu+u_{i M V}^{(r)}} \mathbf{X}_{i}^{\top}\left\{\boldsymbol{\Sigma}_{i M V}^{(r)}\right\}^{-1} \mathbf{Y}_{i}\right]
$$

em que $u_{i M V}^{(r)}=\left(\mathbf{Y}_{i}-\mathbf{X}_{i} \boldsymbol{\beta}_{M V}^{(r)}\right)^{\top} \boldsymbol{\Sigma}_{i V}^{(r+1)^{-1}}\left(\mathbf{Y}_{i}-\mathbf{X}_{i} \boldsymbol{\beta}_{M V}^{(r)}\right)$.

\section{Método de máxima verossimilhança restrita}

Seja $\boldsymbol{\theta}_{M V R}^{(r)}=\left(\boldsymbol{\alpha}_{M V R}{ }^{(r)}, \boldsymbol{\gamma}_{M V R}^{(r)}\right)$ e $\boldsymbol{\beta}_{M V R}^{(r)}$ a estimativa de máxima verossimilhança restrita do vetor $\boldsymbol{\alpha}$ obtida na $r$-ésima iteração. Então, na iteração $(r+1)$ temos que:

- O vetor $\boldsymbol{\theta}_{M V R}^{(r)}$ é considerado o valor inicial e $\boldsymbol{\theta}_{M V R}^{(r+1)}$ será obtido da maximização da função dada na equação (4.18) em que $\mathbf{b}_{i}=\left(\mathbf{X}_{i}^{\top} \boldsymbol{\Sigma}_{i}^{-1} \mathbf{X}_{i}\right)^{-1} \mathbf{X}_{i}^{\top} \boldsymbol{\Sigma}_{i}^{-1} \mathbf{Y}_{i}$ e $u_{i 2}=\left(\mathbf{Y}_{i}-\mathbf{X}_{i} \mathbf{b}_{i}\right)^{\top} \boldsymbol{\Sigma}_{i}^{-1}\left(\mathbf{Y}_{i}-\mathbf{X}_{i} \mathbf{b}_{i}\right)$.

- Uma vez obtida a estimativa restrita do vetor $\boldsymbol{\theta}$, devemos resolver um sistema de equações para obter a estimativa restrita do vetor $\boldsymbol{\beta}$, dada por

$$
\boldsymbol{\beta}_{M V R}^{r+1}=\left(\sum_{i=1}^{n} \frac{\nu+m_{i}}{\nu+u_{i M V R}^{(r)}} \mathbf{X}_{i}^{\top} \widehat{\boldsymbol{\Sigma}}_{i M V R}^{-1} \mathbf{X}_{i}\right)^{-1}\left(\sum_{i=1}^{n} \frac{\nu+m_{i}}{\nu+u_{i M V R}^{(r)}} \mathbf{X}_{i}^{\top} \widehat{\boldsymbol{\Sigma}}_{i M V R}^{-1} \mathbf{Y}_{i}\right),
$$

em que $\widehat{\boldsymbol{\Sigma}}_{i M V R}$ denota a estimativa restrita da matriz $\mathbf{R}$ resultante no processo iterativo anterior e $u_{i M V R}^{(r)}=\left(\mathbf{Y}_{i}-\mathbf{X}_{i} \boldsymbol{\beta}_{M V R}^{(r)}\right)^{\top} \widehat{\boldsymbol{\Sigma}}_{i M V R}^{-1}\left(\mathbf{Y}_{i}-\mathbf{X}_{i} \boldsymbol{\beta}_{M V R}^{(r)}\right)$.

\section{Método de máxima verossimilhança perfilada modificada}

Consideremos o vetor $\boldsymbol{\theta}_{M V P M}^{(r)}$ como a estimativa de máxima verossimilhança perfilada modificada obtida na $r$-ésima iteração. Portanto, na $(r+1)$-iteração temos:

- O vetor $\boldsymbol{\theta}_{M V P M}^{(r+1)}$ é obtido da maximização de função de verossimilhança perfilada modificada expressa na equação (4.20), considerando $\boldsymbol{\theta}_{M V P M}^{(r)}$ como o valor inicial, sendo $\hat{\boldsymbol{\beta}}_{\theta}$ a estimativa de máxima verossimilhança obtida no proceso iterativo de máxima verossimilhança. 
- Estimativa do vetor de $\boldsymbol{\beta}$ na $(r+1)$-iteração é a solução do sistema de equações dado por

$$
\boldsymbol{\beta}_{M V P M}^{r+1}=\left(\sum_{i=1}^{n} \frac{\nu+m_{i}}{\nu+u_{i M V P M}^{(r)}} \mathbf{X}_{i}^{\top} \widehat{\boldsymbol{\Sigma}}_{i M V P M}^{-1} \mathbf{X}_{i}\right)^{-1}\left(\sum_{i=1}^{n} \frac{\nu+m_{i}}{\nu+u_{i M V P M}^{(r)}} \mathbf{X}_{i}^{\top} \widehat{\boldsymbol{\Sigma}}_{i M V P M}^{-1} \mathbf{Y}_{i}\right),
$$

em que $u_{i M V P M}^{(r)}$ é dada por $\left(\mathbf{Y}_{i}-\mathbf{X}_{i} \boldsymbol{\beta}_{\theta}^{(r)}\right)^{\top} \widehat{\boldsymbol{\Sigma}}_{i M V P M}^{-1}\left(\mathbf{Y}_{i}-\mathbf{X}_{i} \boldsymbol{\beta}_{\theta}^{(r)}\right)$ e $\widehat{\boldsymbol{\Sigma}}_{i M V P M}$ denota a estimativa de máxima verossimilhança perfilada modificada da matriz $\boldsymbol{\Sigma}_{i}$.

\section{Método de máxima verossimilhança perfilada modificada aproximada}

Similar ao caso anterior temos que a função de verossimilhança perfilada modificada aproximada é dada na equação (4.21) e considerando os vetores $\boldsymbol{\theta}_{M V P M A}^{(r)}$ e $\boldsymbol{\beta}_{M V P M A}^{(r)}$ como as estimativas de máxima verossimilhança perfilada modificada aproximada obtidas na $r$-ésima iteração, temos que o processo na $(r+1)$-iteração fica dado por:

- Obter o vetor $\boldsymbol{\theta}_{M V P M A}^{(r+1)}$ da maximização de função de verossimilhança perfilada modificada considerando o vetor $\boldsymbol{\theta}_{M V P M A}^{(r)}$ como valor inicial.

- A atualização da estimativa do vetor $\boldsymbol{\beta}$ na $(r+1)$-iteração é dado por $\boldsymbol{\beta}_{M V P M A}^{r+1}=\left(\sum_{i=1}^{n} \frac{\nu+m_{i}}{\nu+u_{i M V P M A}^{(r)}} \mathbf{X}_{i}^{\top} \widehat{\boldsymbol{\Sigma}}_{i M V P M A}^{-1} \mathbf{X}_{i}\right)^{-1}\left(\sum_{i=1}^{n} \frac{\nu+m_{i}}{\nu+u_{i M V P M A}^{(r)}} \mathbf{X}_{i}^{\top} \widehat{\boldsymbol{\Sigma}}_{i M V P M A}^{-1} \mathbf{Y}_{i}\right)$,

em que $\widehat{\boldsymbol{\Sigma}}_{i M V P M A}$ denota estimativa de máxima verossimilhança perfilada modificada aproximada da matriz $\boldsymbol{\Sigma}_{i}$. 


\section{Apêndice E}

\section{Aproximação parcial de Laplace}

Nesta seção estendemos a aproximação parcial de Laplace, desenvolvida por Taylor e Verbyla (2006) no modelo linear heteroscedastico t-Student, para o modelo apresentado no Capítulo 5. Considerando a formulação do modelo descrito na Seção 5.1, podemos escrever as seguintes funções:

$$
h_{1}(\mathbf{Y}, \boldsymbol{\omega} ; \boldsymbol{\lambda})=\log \left\{f_{\mathbf{V}_{1} \mid \mathbf{V}_{2}}\left(\mathbf{v}_{1} \mid \mathbf{v}_{2}, \boldsymbol{\omega} ; \boldsymbol{\beta}, \boldsymbol{\theta}, \nu\right)\right\}
$$

e

$$
h_{2}(\mathbf{Y}, \boldsymbol{\omega} ; \boldsymbol{\eta})=\log \left\{f_{\mathbf{V}_{2}}\left(\mathbf{v}_{2} \mid \boldsymbol{\omega} ; \boldsymbol{\theta}\right)\right\}+\log \left\{f_{\boldsymbol{\omega}}(\boldsymbol{\omega} ; \nu)\right\} .
$$

A função $h_{1}(\cdot)$ depende do vetor completo do modelo e a função $h_{2}(\cdot)$ depende dos parâmetros de interesse $\boldsymbol{\eta}$. Lembrando que $\boldsymbol{\eta}$ é um subconjunto de parâmetros dado por $(\boldsymbol{\theta}, \nu)$. Assim, a função de verossimilhança marginal $\mathbf{Y}$ dada na equação (5.3) pode ser expressada como

$$
L(\boldsymbol{\lambda} ; \mathbf{Y})=\int_{\mathbb{R}^{n}} \exp \left\{h_{1}(\mathbf{Y}, \boldsymbol{\omega} ; \boldsymbol{\lambda})+h_{2}(\mathbf{Y}, \boldsymbol{\omega} ; \boldsymbol{\eta})\right\} d \boldsymbol{\omega} .
$$

Esta partição permite utilizar a expansão de série de Taylor de integrais em torno do máximo de $\boldsymbol{\omega}$ em $h_{2}(\cdot)$, denominado $\tilde{\boldsymbol{\omega}}$. Então, a derivada parcial da função $h_{2}(\cdot)$ com respeito a $\boldsymbol{\omega}$ avaliada em $\tilde{\boldsymbol{\omega}}$ é zero, denotada por $h_{2}^{\prime}(\tilde{\boldsymbol{\omega}})=0$ e a função de verossimilhança marginal $\mathbf{Y}$ aproximadamente pode ser escrita como

$$
\begin{aligned}
L(\boldsymbol{\lambda} ; \mathbf{Y}) \approx & \exp \left\{h_{1}(\mathbf{Y}, \tilde{\boldsymbol{\omega}} ; \boldsymbol{\lambda})+h_{2}(\mathbf{Y}, \tilde{\boldsymbol{\omega}} ; \boldsymbol{\eta})\right\} \int_{\mathbb{R}^{n}} \exp \left[(\boldsymbol{\omega}-\tilde{\boldsymbol{\omega}})^{\top} h_{1}^{\prime}(\mathbf{Y}, \tilde{\boldsymbol{\omega}} ; \boldsymbol{\lambda})\right. \\
& \left.-\frac{1}{2}(\boldsymbol{\omega}-\tilde{\boldsymbol{\omega}})^{\top}\left\{-H_{1}(\mathbf{Y}, \boldsymbol{\omega} ; \boldsymbol{\lambda})\right\}(\boldsymbol{\omega}-\tilde{\boldsymbol{\omega}})-\frac{1}{2}(\boldsymbol{\omega}-\tilde{\boldsymbol{\omega}})^{\top}\left\{-H_{2}(\mathbf{Y}, \boldsymbol{\omega} ; \boldsymbol{\eta})\right\}(\boldsymbol{\omega}-\tilde{\boldsymbol{\omega}})\right] d \boldsymbol{\omega},
\end{aligned}
$$

em que $h_{1}^{\prime}(\mathbf{Y}, \tilde{\boldsymbol{\omega}} ; \boldsymbol{\lambda})$ é um vetor de derivadas parciais com $i$-ésimo elemento $\partial h_{1}(\cdot) / \partial \omega_{i}$ e $H_{1}(\mathbf{Y}, \boldsymbol{\omega} ; \boldsymbol{\lambda})$ é a matriz das segundas derivadas parciais com respeito a $\boldsymbol{\omega}$ com elemento $(i, j), \partial^{2} h_{1}(\cdot) / \partial \omega_{i} \partial \omega_{j}$. Da mesma forma, temos que $h_{2}^{\prime}(\mathbf{Y}, \tilde{\boldsymbol{\omega}} ; \boldsymbol{\eta})$ é um vetor de derivadas parciais com $i$-ésimo elemento $\partial h_{2}(\cdot) / \partial \omega_{i}$ e $H_{2}(\mathbf{Y}, \boldsymbol{\omega} ; \boldsymbol{\eta})$ é a matriz das segundas derivadas parciais com respeito a $\boldsymbol{\omega}$ com elemento $(i, j)$ igual a $\partial^{2} h_{2}(\cdot) / \partial \omega_{i} \partial \omega_{j}$. Completando os quadrados dos componentes, temos

$$
\begin{aligned}
L(\boldsymbol{\lambda} ; \mathbf{Y}) \approx & \exp \left\{h_{1}(\mathbf{Y}, \tilde{\boldsymbol{\omega}} ; \boldsymbol{\lambda})+h_{2}(\mathbf{Y}, \tilde{\boldsymbol{\omega}} ; \boldsymbol{\eta})\right\} \exp \left[h_{1}^{\prime}(\mathbf{Y}, \tilde{\boldsymbol{\omega}} ; \boldsymbol{\lambda})^{\top}\{M(\mathbf{Y}, \tilde{\boldsymbol{\omega}} ; \boldsymbol{\eta})\}^{-1} h_{1}^{\prime}(\mathbf{Y}, \tilde{\boldsymbol{\omega}} ; \boldsymbol{\lambda})\right] \\
& \times \int_{\mathbb{R}^{n}} \exp \left\{-\frac{1}{2} a(\mathbf{Y}, \boldsymbol{\omega} ; \boldsymbol{\eta})^{\top} M(\mathbf{Y}, \tilde{\boldsymbol{\omega}} ; \boldsymbol{\eta}) a(\mathbf{Y}, \boldsymbol{\omega} ; \boldsymbol{\eta})\right\} d \boldsymbol{\omega}
\end{aligned}
$$

em que

$$
a(\mathbf{Y}, \boldsymbol{\omega} ; \boldsymbol{\eta})=(\boldsymbol{\omega}-\tilde{\boldsymbol{\omega}})-\{M(\mathbf{Y}, \tilde{\boldsymbol{\omega}} ; \boldsymbol{\eta})\}^{-1} h_{1}^{\prime}(\mathbf{Y}, \tilde{\boldsymbol{\omega}} ; \boldsymbol{\lambda})
$$


e

$$
M(\mathbf{Y}, \tilde{\boldsymbol{\omega}} ; \boldsymbol{\eta})=-H_{1}(\mathbf{Y}, \boldsymbol{\omega} ; \boldsymbol{\lambda})-H_{2}(\mathbf{Y}, \boldsymbol{\omega} ; \boldsymbol{\eta}) .
$$

A última integral da equação (E.1) tem forma padrão e pode ser considerada como uma função de densidade de distribuição normal $n$-variada com vetor de média $\left[\tilde{\boldsymbol{\omega}}-\{M(\mathbf{Y}, \tilde{\boldsymbol{\omega}} ; \boldsymbol{\eta})\}^{-1} h_{1}^{\prime}(\mathbf{Y}, \tilde{\boldsymbol{\omega}} ; \boldsymbol{\lambda})\right]$ e matriz de variância-covariância $M(\mathbf{Y}, \tilde{\boldsymbol{\omega}} ; \boldsymbol{\eta})$. Assim, completando a integral da verossimilhança marginal fica

$$
\begin{aligned}
L(\boldsymbol{\lambda} ; \mathbf{Y}) \approx & (2 \pi)^{n / 2}|M(\mathbf{Y}, \tilde{\boldsymbol{\omega}} ; \boldsymbol{\eta})|^{-1 / 2} \exp \left\{h_{1}(\mathbf{Y}, \tilde{\boldsymbol{\omega}} ; \boldsymbol{\lambda})\right. \\
& \left.+h_{2}(\mathbf{Y}, \tilde{\boldsymbol{\omega}} ; \boldsymbol{\eta})\right\} \exp \left[h_{1}^{\prime}(\mathbf{Y}, \tilde{\boldsymbol{\omega}} ; \boldsymbol{\lambda})^{\top}\{M(\mathbf{Y}, \tilde{\boldsymbol{\omega}} ; \boldsymbol{\eta})\}^{-1} h_{1}^{\prime}(\mathbf{Y}, \tilde{\boldsymbol{\omega}} ; \boldsymbol{\lambda})\right] .
\end{aligned}
$$

Se $\tilde{\boldsymbol{\omega}}$ está na vizinhança do máximo de $h_{1}(\cdot)+h_{2}(\cdot)$, então a primeira exponencial seria próxima a zero e portanto a aproximação seria aproximação de Laplace ordinária. Como consideramos o máximo de $\boldsymbol{\omega}$ em $h_{2}(\cdot)$, a última expressão é denominada aproximação de Laplace parcial da verossimilhança marginal e pode ser expressa como

$$
L(\boldsymbol{\lambda} ; \mathbf{Y}) \approx(2 \pi)^{n / 2} L_{1}(\boldsymbol{\lambda} ; \mathbf{Y}) L_{2}(\boldsymbol{\eta} ; \mathbf{Y}),
$$

em que

$$
\begin{aligned}
L_{1}(\boldsymbol{\lambda} ; \mathbf{Y})= & \left|I_{n}+\left\{-H_{2}(\mathbf{Y}, \boldsymbol{\omega} ; \boldsymbol{\eta})\right\}^{-1}\left\{-H_{1}(\mathbf{Y}, \boldsymbol{\omega} ; \boldsymbol{\lambda})\right\}\right|^{-1 / 2} \exp \left\{h_{1}(\mathbf{Y}, \tilde{\boldsymbol{\omega}} ; \boldsymbol{\lambda})\right\} \\
& \times \exp \left[h_{1}^{\prime}(\mathbf{Y}, \tilde{\boldsymbol{\omega}} ; \boldsymbol{\lambda})^{\top}\{M(\mathbf{Y}, \tilde{\boldsymbol{\omega}} ; \boldsymbol{\eta})\}^{-1} h_{1}^{\prime}(\mathbf{Y}, \tilde{\boldsymbol{\omega}} ; \boldsymbol{\lambda})\right]
\end{aligned}
$$

$\mathrm{e}$

$$
L_{2}(\boldsymbol{\eta} ; \mathbf{Y})=\left|-H_{2}(\mathbf{Y}, \boldsymbol{\omega} ; \boldsymbol{\eta})\right|^{-1 / 2} \exp \left\{h_{2}(\mathbf{Y}, \tilde{\boldsymbol{\omega}} ; \boldsymbol{\eta})\right\} .
$$

Como $L_{2}(\boldsymbol{\eta}, \mathbf{Y})$ não depende de $\boldsymbol{\beta}$, pode ser considerada uma aproximação de MVR dos parâmetros $\boldsymbol{\eta}$. Na próxima seção consideramos esta aproximação parcial de Laplace para o modelo misto linear t-Student apresentado na equação (5.2).

\section{Efeitos escala no modelo linear misto t-Student}

Consideramos $\boldsymbol{\omega}$ como efeitos escala do modelo (5.2). Utilizando a metodologia padrão, esses efeitos podem ser preditos considerando a distribuição condicional de $\omega_{i} \mid \mathbf{Y}_{i}$, que neste caso é dada por

$$
\omega_{i} \mid \mathbf{Y}_{i} \sim \operatorname{Gamma}\left(\frac{\nu+m_{i}}{2}, \frac{\nu+d_{i}}{2}\right),
$$

para $i=1, \ldots, m$. O método usual para obter um preditor de $\boldsymbol{\omega}$ é encontrar o melhor preditor linear não viesado (BLUP) de $\omega_{i}$, dado por

$$
\bar{\omega}_{i}=\mathrm{E}\left(\omega_{i} \mid \mathbf{Y}_{i}\right)=\frac{\nu+m_{i}}{\nu+d_{i}} .
$$

Vemos que este último resultado difere do máximo de $\tilde{\omega}_{i}$ de $h_{2}(\cdot)$. Na Tese de Doutorado de Taylor (2005) sugere analisar a transformação $\omega_{i}^{*}=\log \left(\omega_{i}\right)$. A justificativa vem do fato de Lee e Nelder (1996) e Lee e Nelder (2001) mencionarem que o uso da escala correta, a escala em que os efeitos aleatórios ocorrem linearmente, garante que a estimativa dos efeitos aleatórios obtidos em modelos lineares generalizados hierárquicos será equivalente à estimativa dos efeitos aleatórios preditos obtidos da distribuição condicional de $\omega_{i} \mid \mathbf{Y}_{i}$.

Analisando a transformação proposta, temos que a distribuição de $\boldsymbol{\omega}^{*}$ é conhecida como a distri- 
buição log-gamma e o logaritmo de sua verossimilhança fica dado por

$$
l\left(\nu ; \boldsymbol{\omega}^{*}\right)=\frac{n \nu}{2} \log \frac{\nu}{2}-n \log \Gamma(\nu / 2)+\sum_{i=1}^{n}\left(\frac{\nu}{2} \omega_{i}^{*}-\frac{\nu}{2} \exp \omega_{i}^{*}\right) .
$$

Neste caso, a função $h_{2}(\cdot)$ assume a forma

$$
\begin{aligned}
h_{2}\left(\mathbf{Y}, \boldsymbol{\omega}^{*} ; \boldsymbol{\eta}\right)= & \log \left\{f_{\mathbf{V}_{2}}\left(\mathbf{v}_{2} \mid \boldsymbol{\omega}^{*} ; \boldsymbol{\theta}\right)\right\}+\log \left\{f_{\boldsymbol{\omega}^{*}}\left(\boldsymbol{\omega}^{*} ; \nu\right)\right\} \\
= & -\frac{1}{2}\left\{(n-p) \log (2 \pi)+\log \left|\boldsymbol{\Sigma}^{*}\right|+\log \left|\mathbf{X}^{\top} \boldsymbol{\Sigma}^{*-1} \mathbf{X}\right|+\mathbf{Y}^{\top} \mathbf{P}^{*} \mathbf{Y}\right\} \\
& +\frac{n \nu}{2} \log \frac{\nu}{2}-n \log \Gamma(\nu / 2)+\sum_{i=1}^{n}\left(\frac{\nu}{2} \omega_{i}^{*}-\frac{\nu}{2} \exp \omega_{i}^{*}\right),
\end{aligned}
$$

em que $\boldsymbol{\Sigma}^{*}$ e $\mathbf{P}^{*}$ denotam $\boldsymbol{\Sigma}$ e $\mathbf{P}$ avaliados em $\boldsymbol{\omega}^{*}$ ao invés de $\boldsymbol{\omega}$. A decomposição da aproximação parcial de Laplace, leva ao seguinte resultado:

$$
\begin{aligned}
L_{1}^{*}(\boldsymbol{\lambda} ; \mathbf{Y})= & \left|\mathbf{I}_{n}+\left(\mathbf{V}^{*}-\widetilde{\mathbf{H}}^{* 2}-2 \widetilde{\mathbf{R}}_{c}^{\top} \widetilde{\mathbf{H}} \widetilde{\mathbf{R}}_{c}\right)^{-1}\left(\widetilde{\mathbf{H}}^{* 2}+2 \widetilde{\mathbf{R}}_{c}^{\top} \widetilde{\mathbf{H}} \widetilde{\mathbf{R}}_{c}-\mathbf{V}^{*}+\nu \widetilde{\mathbf{\Omega}}\right)\right|^{-1 / 2} \\
& \exp \left\{-\frac{1}{2}\left(p \log (2 \pi)-\log \left|\mathbf{X}^{\top} \widetilde{\boldsymbol{\Sigma}}^{-1} \mathbf{X}\right|+(\mathbf{Y}-\mathbf{X} \boldsymbol{\beta})^{\top} \widetilde{\boldsymbol{\Sigma}}^{-1}(\mathbf{Y}-\mathbf{X} \boldsymbol{\beta})-\mathbf{Y}^{\top} \widetilde{\mathbf{P}} \mathbf{Y}\right)\right\} \\
& \exp \left\{-\frac{1}{4} \mathbf{1}^{\top}\left(\widetilde{\mathbf{H}}^{*}-\widetilde{\mathbf{D}}+\widetilde{\mathbf{D}}_{c}\right)^{\top}(\widetilde{\mathbf{D}}+\nu \widetilde{\mathbf{\Omega}})\left(\widetilde{\mathbf{H}}^{*}-\widetilde{\mathbf{D}}+\widetilde{\mathbf{D}}_{c}\right) \mathbf{1}\right\}
\end{aligned}
$$

e

$$
\begin{aligned}
L_{2}^{*}(\boldsymbol{\eta} ; \mathbf{Y})= & |\widetilde{\boldsymbol{\Sigma}}|^{-1 / 2}\left|\mathbf{X}^{\top} \widetilde{\boldsymbol{\Sigma}}^{-1} \mathbf{X}\right|^{-1 / 2} \|\left.\frac{1}{2}\left(\mathbf{V}^{*}-\widetilde{\mathbf{H}}^{* 2}-2 \widetilde{\mathbf{R}}_{c}^{\top} \widetilde{\mathbf{H}} \widetilde{\mathbf{R}}_{c}\right)\right|^{-1 / 2}(2 \pi)^{-(n-p) / 2} \exp \left\{-\frac{1}{2} \mathbf{Y}^{\top} \widetilde{\mathbf{P}} \mathbf{Y}\right\} \\
& \times \frac{(\nu / 2)^{n \nu / 2}}{\Gamma(\nu / 2)^{n}} \exp \left\{-\frac{\nu}{2} \sum_{i=1}^{n} \tilde{\omega}_{i}\right\} \prod_{i=1}^{n} \tilde{\omega}_{i}^{\nu / 2-1},
\end{aligned}
$$

em que $\mathbf{V}^{*}=\left(\nu+m_{i}\right) \mathbf{I}_{n}$ e o máximo de $\omega_{i}=\exp \left(\omega_{i}^{*}\right)$ em $h_{2}(\cdot)$ é dado por

$$
\tilde{\omega}_{i}=\frac{\nu+m_{i}-\operatorname{tr}\left(\widetilde{\mathbf{H}}_{i i}\right)}{\nu+\tilde{d}_{i, c}}
$$

Vemos que $L_{1}^{*}(\boldsymbol{\lambda} ; \mathbf{Y})$ difere de $L_{1}(\boldsymbol{\lambda} ; \mathbf{Y})$, ou seja, a aproximação da verossimilhança condicional para os parâmetros de locação não é invariante sob mudanças dos efeitos aleatórios de escala. De mesma forma, $L_{2}^{*}(\boldsymbol{\eta} ; \mathbf{Y})$ é diferente de $L_{2}(\boldsymbol{\eta} ; \mathbf{Y})$. Portanto, a mudança altera a aproximação MVR. Na Tese de Doutorado de Taylor (2005) considera a estimação a partir das verossimilhanças aproximadas sem a mudança dos efeitos de escala. 
APÊNDICE E 


\section{Apêndice F}

\section{Tabelas}

\section{F.1 Tabelas de resultados das aplicações}

Tabela F.1: Estimativas bootstrap dos parâmetros e seus desvio padrão para o modelo normal e modelo t-Student, obtidas pelos diferentes métodos de estimação, considerando o procedimento de reamostragem em que todos os distritos têm a mesma probabilidade de ser escolhido.

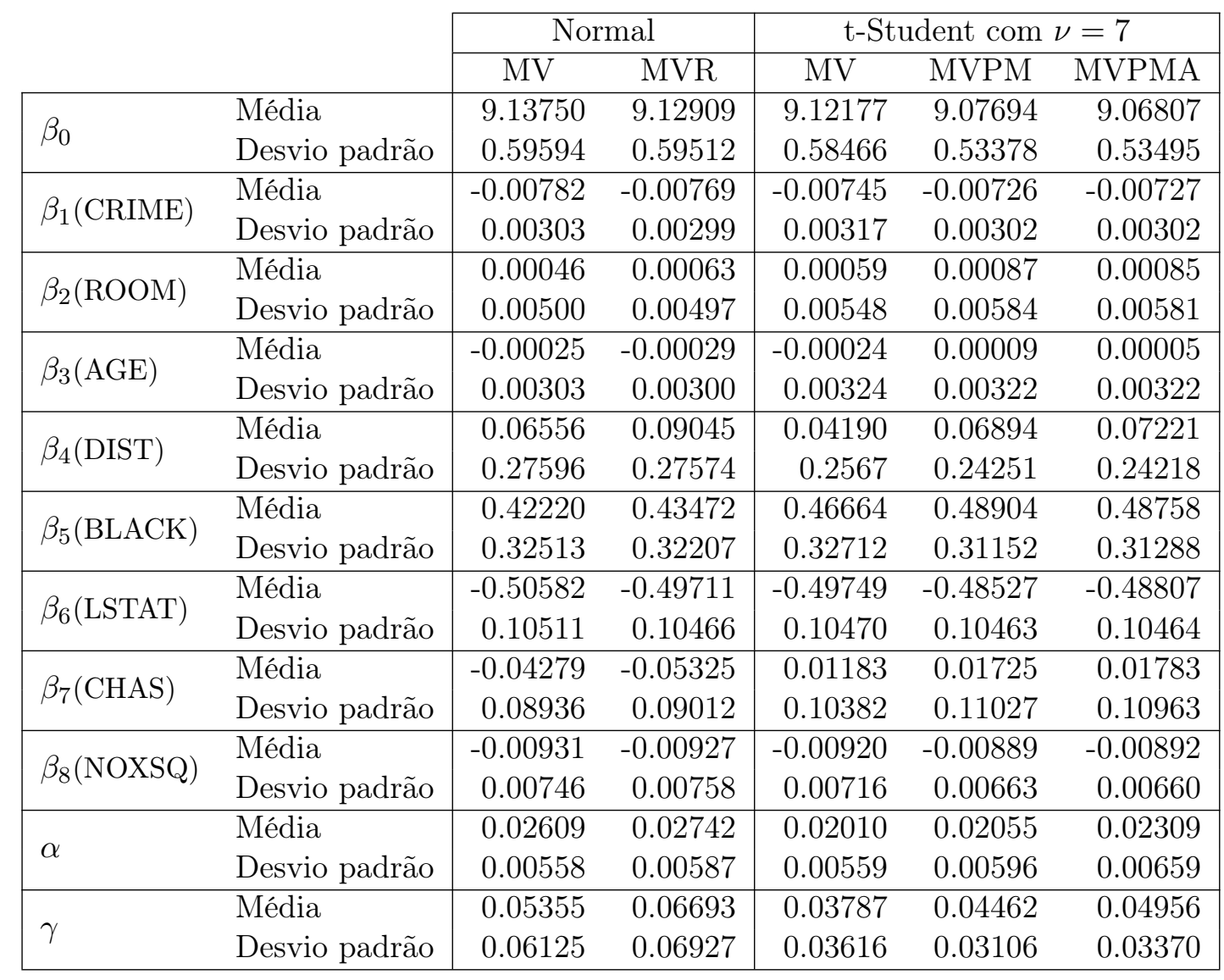


Tabela F.2: Estimativas bootstrap dos parâmetros e seus desvio padrão para o modelo normal e modelo t-Student, obtidas pelos diferentes métodos de estimação, considerando o procedimento de reamostragem em que os distritos de maior tamanho têm uma maior probabilidade de ser escolhido.

\begin{tabular}{|c|c|c|c|c|c|c|}
\hline & \multicolumn{2}{|c|}{ Normal } & \multicolumn{3}{|c|}{ t-Student $\operatorname{com} \nu=7$} \\
\hline & & MV & MVR & MV & MVPM & MVPMA \\
\hline \multirow{2}{*}{$\beta_{0}$} & Média & 8.58187 & 8.57864 & 8.57232 & 8.54725 & 8.54319 \\
\hline & Desvio padrão & 0.41738 & 0.41240 & 0.41365 & 0.38944 & 0.38657 \\
\hline \multirow{2}{*}{$\beta_{1}(\mathrm{CRIME})$} & Média & -0.00681 & -0.00674 & -0.00657 & -0.00648 & -0.00648 \\
\hline & Desvio padrão & 0.00202 & 0.00200 & 0.00226 & 0.00221 & 0.00222 \\
\hline \multirow{2}{*}{$\beta_{2}(\mathrm{ROOM})$} & Média & 0.00032 & 0.00040 & 0.00041 & 0.00064 & 0.00064 \\
\hline & Desvio padrão & 0.00463 & 0.00460 & 0.00501 & 0.00540 & 0.00537 \\
\hline \multirow{2}{*}{$\beta_{3}(\mathrm{AGE})$} & Média & 0.00167 & 0.00161 & 0.00161 & 0.0016 & 0.00164 \\
\hline & Desvio padrão & 0.00257 & 0.00255 & 0.00275 & 0.00286 & 0.00286 \\
\hline \multirow{2}{*}{$\beta_{4}(\mathrm{DIST})$} & Média & 0.33078 & 0.34762 & 0.30746 & 0.32202 & 0.32437 \\
\hline & Desvio padrão & 0.20832 & 0.20437 & 0.20000 & 0.18729 & 0.18667 \\
\hline \multirow{2}{*}{$\beta_{5}(\mathrm{BLACK})$} & Média & 0.48677 & 0.49576 & 0.51909 & 0.53586 & 0.53528 \\
\hline & Desvio padrão & 0.16813 & 0.16547 & 0.16666 & 0.16009 & 0.16086 \\
\hline \multirow{2}{*}{$\beta_{6}(\mathrm{LSTAT})$} & Média & -0.54365 & -0.5375 & -0.53546 & -0.52354 & -0.52454 \\
\hline & Desvio padrão & 0.09121 & 0.09074 & 0.09215 & 0.09507 & 0.09447 \\
\hline \multirow{2}{*}{$\beta_{7}(\mathrm{CHAS})$} & Média & -0.01599 & -0.02315 & 0.01860 & 0.01126 & 0.01014 \\
\hline & Desvio padrão & 0.09431 & 0.09504 & 0.11243 & 0.11700 & 0.11595 \\
\hline \multirow{2}{*}{$\beta_{8}(\mathrm{NOXSQ})$} & Média & -0.00819 & -0.00813 & -0.00782 & -0.00738 & -0.00743 \\
\hline & Desvio padrão & 0.00504 & 0.00508 & 0.00517 & 0.00518 & 0.00514 \\
\hline \multirow{2}{*}{$\alpha$} & Média & 0.02461 & 0.02566 & 0.02061 & 0.02083 & 0.02301 \\
\hline & Desvio padrão & 0.00433 & 0.00460 & 0.00449 & 0.00492 & 0.00539 \\
\hline \multirow{2}{*}{$\gamma$} & Média & 0.03845 & 0.04671 & 0.01811 & 0.02155 & 0.02410 \\
\hline & Desvio padrão & 0.03443 & 0.03964 & 0.01590 & 0.01705 & 0.01898 \\
\hline
\end{tabular}




\section{Referências Bibliográficas}

Akaike(1974) H. Akaike. A new look at statistical models identification. IEEE Trans Autom Control, 19:716-722. Citado na pág. 75, 80

Anderson et al.(1986) T.W. Anderson, K.T. Fang e H. Hsu. Maximum-likelihood estimates and likelihood ratio criteria for multivariate elliptically contoured distributions. The Canadian Journal of Statistics, 14:55-59. Citado na pág. 2, 39, 85

Arellano(1994) R. Arellano. Elliptical Distributions: Properties, Inference and Applications in Regression Models. Tese de Doutorado, Universidade de São Paulo, Brasil. Citado na pág. 15, 18, 105

Barndorff-Nielsen(1980) O.E. Barndorff-Nielsen. Conditionality resolutions. Biometrika, 67: 293-310. Citado na pág. 89, 90

Barndorff-Nielsen(1983) O.E. Barndorff-Nielsen. On a formulae for the distribution of the maximum likelihood estimator. Biometrika, 70:343-365. Citado na pág. 10, 90

Basu(1977) D. Basu. On the elimination of nuisance parameters. Journal of the American Statistical Association, 72:355-366. Citado na pág. 1

Belsley et al.(1980) D. A. Belsley, E. Kuh e R. E. Welsch. Regression Diagnostics: Identifying Influence. Data and Sources of Collinearity. Wiley, $1 \underline{a}$ edição. Citado na pág. 77

Berkane et al.(1994) M. Berkane, Bentler P.M. e Y. Kano. Pseudo maximum likelihood estimation in elliptical theory: Effects of misspecification. Computational Statistics and Data Analysis, 18: 255-267. Citado na pág. 2

Borssoi(2013) J.A . Borssoi. Modelos Mistos Lineares Elípticos com Erros de Medição. Tese de Doutorado, Universidade de São Paulo, Brasil. Citado na pág. 77, 78

Borssoi et al.(2017) J.A . Borssoi, G.A. Paula e M. Galea. Elliptical linear mixed models with a covariate subject to measurement error. Statistical Papers, (aceito para publicação). Citado na pág. $2,77,80$

Carstensen et al.(2015) B. Carstensen, L. Gurrin, C. Ekstrom e M. Figurski. MethComp: Functions for Analysis of Agreement in Method Comparison Studies, 2015. URL https://CRAN. R-project.org $/$ package=MethComp. R package version 1.22.2. Citado na pág. 74

Choudhary e Nagaraja(2017) P.K. Choudhary e H.N. Nagaraja. Measuring Agreement: Models, Methods, and Applications. Wiley Series in Probability and Statistics. John Wiley \& Sons, Incorporated, $1 \underline{a}$ edição. Citado na pág. 74

Copper e Thompson(1977) D.M. Copper e R. Thompson. A note on the estimation of the parameters of the autoregressive-moving average process. The American Statistician, 64:625628. Citado na pág. 1 
Corbeil e Searle(1976) R.R. Corbeil e S.R. Searle. Restricted maximum likelihood (reml) estimation of variance components in the mixed model. Journal of the American Statistical Association, 18(1):31-38. Citado na pág. 1

Cotes et al.(1986) P. M. Cotes, C. J. Doré, J. A. Liu Yin, S. M. Lewis, M. Messinezy, T. C. Pearson e C. Reid. Determination of serum immunoreactive erythropoietin in the investigation of erythrocytosis. New England Journal of Medicine, 315-5:283-287. Citado na pág. 74

Cox e Reid(1987) D.R. Cox e N. Reid. Parameter orthogonality and approximate conditional inference. Journal of the Royal Statistical Society, B 49:1-39. Citado na pág. 10, 40, 90

Cressie(1993) N. A. C. Cressie. Statistics for Spatial Data. Wiley, $2^{a}$ edição. Citado na pág. 1

Demidenko(2013) E. Demidenko. Mixed Models: Theory and Applications with $R$. Wiley, $2^{\underline{a}}$ edição. Citado na pág. 1, 41

Fang et al.(1990) K.T. Fang, S. Kotz e K.W. Ng. Symmetric Multivariate and Related Distributions. Chapman and Hall, $1^{\underline{a}}$ edição. Citado na pág. 2, 15

Gong e Samaniego(1981) G. Gong e F. J. Samaniego. Pseudo-maximum likelihood estimation: Theory and aplications. The Annals of Statistics, 9:861-869. Citado na pág. 2

Gómez et al.(1998) E. Gómez, M.A. Gomez-Villegas e J.M. Marína. A multivariate generalization of the power exponential family of distributions. Communications in Statistics - Theory and Methods, 27:589-600. Citado na pág. 36

Gómez-Sánchez-Manzano et al.(2006) E. Gómez-Sánchez-Manzano, M.A. Gomez-Villegas e J.M. Marína. Sequences of elliptical distributions and mixtures of normal distributions. Journal of Multivariate Analysis, 97:295-310. Citado na pág. 38

Harrison e Rubinfeld(1978) D. Harrison e D. Rubinfeld. Hedonic housing prices and the demand for clean air. Journal of Environmental Economics and Management, 5:81-102. Citado na pág. 77, 81

Harville(1974) D.A. Harville. Bayesian inference for variance components using only error contrasts. Biometrika, 61:383-385. Citado na pág. 1, 7, 10, 43

Harville(1977) D.A. Harville. Maximum likelihood approaches to variance component estimation and to related problems. Journal of the American Statistical Association, 72:320-340. Citado na pág. 1,2

Ibacache-Pulgar et al.(2012) G. Ibacache-Pulgar, G. Paula e M. Galea. Influence diagnostics for elliptical semiparametric mixed models. Statistical Modelling, 12(2):165-193. Citado na pág. 2

James(1973) A.T. James. The variance information manifold and the functions on it. In Multivariate Analysis III, páginas 57-169. Citado na pág. 87

Johnson et al.(1994) N. L. Johnson, S. Kotz e N. Nalakrishnan. Continuous Univariate Distributions. John Wiley, $2^{\underline{a}}$ edição. Citado na pág. 76

Kano et al.(1993) Y. Kano, M. Berkane e Bentler P.M. Statistical inference based on pseudo maximum likelihood in elliptical population. Journal of the American Statistical Association, 88: 135-143. Citado na pág. 2

Laird e Ware(1982) N. M. Laird e J. H. Ware. Random effects models for longitudinal data. Biometrics, 38:963-974. Citado na pág. 41 
Lange et al.(1989) K.L. Lange, R.J. Little e J.M. Taylor. Robust statistical modeling using the t-distribution. Journal of the American Statistical Association, 84(408):881-896. Citado na pág. 2, $6,17,22,34,44,47,76,81,110$

Lee e Nelder(2001) Y. Lee e J. A Nelder. Hierarchical generalized linear models: a synthesis of generalized linear models, random-effect models and structured dispersions. Biometrika, 88: 987-1006. Citado na pág. 118

Lee e Nelder(1996) Y. Lee e J. A. Nelder. Hierarchical generalized linear models. Journal of the American Statistical Association, 58:619-678. Citado na pág. 5, 118

Lee et al.(2006) Y. Lee, J. Nelder e Y. Pawitan. Generalized Linear Models with Random Effects. Chapman \& Hall-CRC, $1^{\underline{a}}$ edição. Citado na pág. 10, 13, 89, 90, 91

Leisch e Dimitriadou(2010) F. Leisch e E. Dimitriadou. mlbench: Machine Learning Benchmark Problems, 2010. URL https://CRAN.R-project.org/package=mlbench. R package version 2.1-1. Citado na pág. 78

Lindsay(1999) B. G Lindsay. Multivariate elliptically-contoured distributions for repeated measurements. Biometrics, 55:1277-1280. Citado na pág. 2

Little(1988) R. J. A Little. Robust estimation of the mean and covariance matrix from data with missing values. Journal of the Royal Statistical Society. Series C (Applied Statistics), 37:23-38. Citado na pág. 76,81

Manghi et al.(2016) R.F. Manghi, G.A. Paula e Cysneiros F.J.A. On elliptical multilevel models. Journal of Applied Statistics, 43(2):2150-2171. Citado na pág. 2

Martin e Altman(1999) B. J. Martin e D. G. Altman. Measuring agreement in method comparison studies. Statistical Methods in Medical Research, 8(2):135-160. Citado na pág. 74

McCullagh e Tibshirani(1990) P. McCullagh e R. Tibshirani. A simple method for the adjustment of profile likelihoods. Journal of the Royal Statistical Society, Serie B, 52:325-344. Citado na pág. 40,56

McGilchrist e Yau(1995) C.A. McGilchrist e K.K.W. Yau. The derivation of blup, ml, reml estimation methods for generalized linear mixed models. Communications in Statistics - Theory and Methods, 24:2963-2980. Citado na pág. 1, 43

Osorio(2018) F. Osorio. heavy: Robust estimation using heavy-tailed distributions, 2018. URL https://CRAN.R-project.org/package=heavy. R package version 0.38.19. Citado na pág. 67

Osorio(2006) F. Osorio. Diagnóstico de Influência em Modelos Elípticos com Efeitos Mistos. Tese de Doutorado, Universidade de São Paulo, Brasil. Citado na pág. 42, 49

Osorio et al.(2007) F. Osorio, G. A. Paula e M. Galea. Assessment of local influence in elliptical linear models with longitudinal structure. Computational Statistics \& Data Analysis, 51(9):43544368. Citado na pág. $2,6,16,17,44$

Patterson e Thompson(1971) H.D. Patterson e R. Thompson. Recovery of inter-block information when block sixes are unequal. Biometrika, 58(3):545-554. Citado na pág. 1, 7, 9, 10, 18

Pinheiro e Bates(2000) J. C. Pinheiro e D. M. Bates. Mixed-Effects Models in S and S-PLUS. Springer, $1^{\underline{a}}$ edição. Citado na pág. 13, 41

Pinheiro et al.(2001) .J.C. Pinheiro, C. Liu e Y.N. Wu. Efficient algorithms for robust estimation in linear mixed-effects models using the multivariate $\mathrm{t} d$ distribution. Journal of Computational and Graphical Statistics, 10(2):249-276. Citado na pág. 2, 57, 60 
R Core Team(2018) R Core Team. R: A Language and Environment for Statistical Computing. R Foundation for Statistical Computing, Vienna, Austria, 2018. URL http://www.R-project.org/. Citado na pág. 63

Russo et al.(2009) C.M. Russo, G.A. Paula e R. Aoki. Influence diagnostics in nonlinear mixedeffects elliptical models. Computational Statistics \& Data Analysis, 53(12):4143-4156. Citado na pág. 2

Russo et al.(2012) C.M. Russo, G.A. Paula, F.J.A. Cysneiros e R. R Aoki. Influence diagnostics in heteroscedastic and/or autoregressive nonlinear elliptical models for correlated data. Journal of Applied Statistics, 39(5):1049-1067. Citado na pág. 2

Savalli et al.(2006) C. Savalli, G.A. Paula e F.J.A. Cysneiros. Assessment of variance components in elliptical linear mixed models. Statistical Modeling, 6:59-76. Citado na pág. 2, 6, 17, 44

Severini(2000) T. A. Severini. Likelihood Methods in Statistics. Oxford Statistical Science Series, $1 \underline{a}$ edição. Citado na pág. 10

Smyth e Verbyla(1999) G. K. Smyth e A. P. Verbyla. Adjusted likelihood methods for modelling dispersion in generalized linear models. Environmetrics, 10:695-709. Citado na pág. 40

Taylor(2005) J. D. Taylor. Scale Parameter Modeling of the t-distribution. Tese de Doutorado, The University of Adelaide Australia. Citado na pág. 118, 119

Taylor e Verbyla(2006) J. D. Taylor e A. P. Verbyla. Asymptotic likelihood approximations using a partial Laplace approximation. Australian and New Zealand Journal of Statistics, 48: 456-476. Citado na pág. 2, 56, 57, 60, 85, 117

Vallejos e Osorio(2015) R. Vallejos e F. Osorio. Effective sample size of spatial process models. Spatial Statistics, 9:66-92. Citado na pág. 2, 39

Verbeke e Molenberghs(2000) G. Verbeke e G. Molenberghs. Linear Mixed Models of Longitudinal Data. Springer Series in Statistics, $1^{\underline{a}}$ edição. Citado na pág. 1, 13, 41, 42

Verbyla(1990) A. P. Verbyla. A conditional derivation of residual maximum likelihood. Journal of the Royal Statistical Society, B36:99-102. Citado na pág. 1, 2, 7, 8, 9, 17, 18, 20, 43, 45, 55, 58, $87,88,93$

Welsh e Richardson(1997) A.H. Welsh e A.M. Richardson. Approaches to the robust estimation of mixed models. Elsevier Science,Handbook of Statistics, 15:43-384. Citado na pág. 2

Zhong et al.(2002) X. P. Zhong, W. K. Fung e B. C. Wei. Estimation in linear models with random effects and errors-in-variables. Annals of the Institute of Statistical Mathematics, 54: 595-606. Citado na pág. 77, 78

Özkale e Acar(2015) M.R. Özkale e T.S. Acar. Leverages and influential observations in a regression model with autocorrelated errors. Communications in Statistics - Theory and Methods, 44:2267-2290. Citado na pág. 64 